\title{
A Highly Convergent Total Synthesis of Norhalichondrin B
}

K. C. Nicolaou, ${ }^{*}$ Saiyong Pan, Yogesh Shelke, ${ }^{\dagger}$ Qiuji Ye, ${ }^{\dagger}$ Dipendu Das, and Stephan Rigol

Department of Chemistry, BioScience Research Collaborative, Rice University, 6100 Main Street, Houston, Texas 77005, United States of America

E-mail: kcn@rice.edu

\section{Table of Contents}

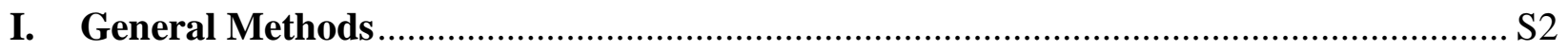

II. Experimental Procedures and Physical Data of Compounds ................................... S3

III. ${ }^{1} \mathrm{H},{ }^{13} \mathrm{C}\left\{{ }^{1} \mathrm{H}\right\}$, COSY, HSQC, HMBC, and nOe NMR Spectra of Compounds............. S57

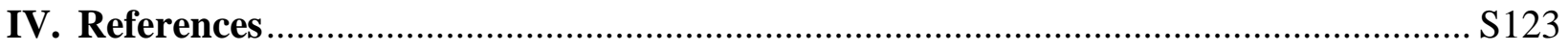




\section{General Information}

All reactions were carried out under an argon atmosphere with dry solvent under anhydrous conditions, unless otherwise noted. Dry acetonitrile (MeCN), dimethylformamide (DMF), dimethyl sulfoxide (DMSO), dichloromethane $\left(\mathrm{CH}_{2} \mathrm{Cl}_{2}\right)$, tetrahydrofuran (THF) and toluene were obtained by passing commercially available pre-dried, oxygen-free formulations through activated alumina columns. Anhydrous benzene, acetone, chloroform $\left(\mathrm{CHCl}_{3}\right)$, methanol $(\mathrm{MeOH})$, ethyl acetate (EtOAc) were purchased from commercial suppliers and stored under argon. Yields refer to chromatographically and spectroscopically ( ${ }^{1} \mathrm{H}$ NMR) homogenous material, unless otherwise stated. Reagents were purchased at the highest commercial quality and used without further purification, unless otherwise noted. Reactions were monitored by thin-layer chromatography (TLC) carried out on S-2 $0.25 \mathrm{~mm}$ E. Merck silica gel plates $\left(60 \mathrm{~F}_{254}\right)$ using UV light for visualization and/or an ethanolic solution of phosphomolybdic acid, an aqueous solution of cerium sulfate or a basic aqueous solution of potassium permanganate as developing agents. E. Merck silica gel (60, particle size $0.040-0.063 \mathrm{~mm}$ ) was used for flash column chromatography. NMR spectra were recorded on a Bruker DRX-600 instrument and calibrated using the resonance signal of the residual undeuterated solvent for ${ }^{1} \mathrm{H}$ NMR $\left[\delta_{\mathrm{H}}=7.26 \mathrm{ppm}\left(\mathrm{CDCl}_{3}\right), \delta_{\mathrm{H}}=7.16 \mathrm{ppm}\left(\mathrm{C}_{6} \mathrm{D}_{6}\right), \delta_{\mathrm{H}}=1.94 \mathrm{ppm}\left(\mathrm{CD}_{3} \mathrm{CN}\right)\right.$, and $\delta_{\mathrm{H}}=3.31 \mathrm{ppm}$ $\left.\left(\mathrm{CD}_{3} \mathrm{OD}\right)\right]$ and deuterated solvent for ${ }^{13} \mathrm{C} \mathrm{NMR}\left[\delta_{\mathrm{C}}=77.16\left(\mathrm{CDCl}_{3}\right), \delta_{\mathrm{C}}=128.06\left(\mathrm{C}_{6} \mathrm{D}_{6}\right), \delta_{\mathrm{C}}=118.26 \mathrm{ppm}\right.$ $\left(\mathrm{CD}_{3} \mathrm{CN}\right)$, and $\left.\delta_{\mathrm{C}}=49.00\left(\mathrm{CD}_{3} \mathrm{OD}\right)\right]$ as an internal reference at $298 \mathrm{~K}$. The following abbreviations were used to designate multiplicities: $\mathrm{s}=$ singlet, $\mathrm{d}=$ doublet, $\mathrm{t}=$ triplet, $\mathrm{q}=$ quartet, quint $=$ quintet, $\mathrm{m}=$ multiplet, br=broad. Infrared (IR) spectra were recorded on a PerkinElmer 100 FT-IR spectrometer. High-resolution

mass spectra (HRMS) were recorded on an Agilent ESI-TOF (time of flight) mass spectrometer using MALDI (matrix-assisted laser desorption ionization) or ESI (electrospray ionization). Optical rotations were measured on a Schmidt+Haensch Polartronic M100 polarimeter at $589.44 \mathrm{~nm}$ using $100 \mathrm{~mm}$ cells and the solvent and concentration indicated, and are reported in units of $10^{-1}\left(\mathrm{deg} \mathrm{cm}^{2} \mathrm{~g}^{-1}\right)$. 


\section{Experimental Procedures and Characterization Data}

[(\{4-[(1-Bromohexan-2-yl)oxy]hex-5-yn-1-yl\}oxy)methyl]benzene (14): To a stirred solution of alkyne

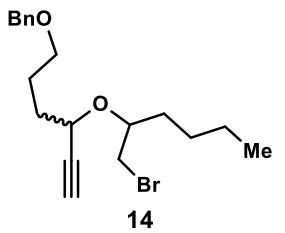

$\mathbf{1 3}^{1}$ (500 mg, $2.45 \mathrm{mmol}, 1.0$ equiv) in $\mathrm{CH}_{2} \mathrm{Cl}_{2}(10 \mathrm{~mL})$ at $23^{\circ} \mathrm{C}$ was added a $\mathrm{Co}_{2}(\mathrm{CO})_{8}$ $\left(1.00 \mathrm{~g}, 2.94 \mathrm{mmol}, 1.2\right.$ equiv) in one portion. After $20 \mathrm{~min}$, a solution of $\mathbf{1 1}^{2}(887 \mathrm{mg}$, $4.89 \mathrm{mmol}, 2.0$ equiv) in $\mathrm{CH}_{2} \mathrm{Cl}_{2}(6 \mathrm{~mL})$ was added. The resulting mixture was cooled to $0{ }^{\circ} \mathrm{C}$, and $\mathrm{BF}_{3} \cdot \mathrm{Et}_{2} \mathrm{O}(604 \mu \mathrm{L}, 4.89 \mathrm{mmol}, 2.0$ equiv $)$ was added dropwise. After $0.5 \mathrm{~h}$, the reaction mixture was carefully quenched by the addition of saturated aqueous $\mathrm{NaHCO}_{3}$ solution $(30 \mathrm{~mL})$, and allowed to warm to $23^{\circ} \mathrm{C}$. The aqueous layer was extracted with $\mathrm{CH}_{2} \mathrm{Cl}_{2}(3 \times 20 \mathrm{~mL})$ and the combined organic layers were dried over anhydrous $\mathrm{Na}_{2} \mathrm{SO}_{4}$, filtered, and concentrated under reduced pressure.

To a stirred solution of the so-obtained crude residue in acetone $(30 \mathrm{~mL})$ was added portion wise diammonium cerium(IV) nitrate (CAN) $\left(6.71 \mathrm{~g}, 12.2 \mathrm{mmol}, 5.0\right.$ equiv) at $0{ }^{\circ} \mathrm{C}$. The resulting mixture was allowed to warm to $23^{\circ} \mathrm{C}$ and stirred for $0.5 \mathrm{~h}$ before it was quenched by the addition of water $(100 \mathrm{~mL})$. The aqueous layer was extracted with EtOAc $(3 \times 50 \mathrm{~mL})$ and the combined organic layers were dried over anhydrous $\mathrm{Na}_{2} \mathrm{SO}_{4}$, filtered, and concentrated under reduced pressure. The obtained residue was purified by flash column chromatography $\left(\mathrm{SiO}_{2}, 5 \rightarrow 20 \%\right.$ ethyl acetate in hexanes) to afford a mixture of diastereomers $14\left(1: 1 d r, 566 \mathrm{mg}, 1.54 \mathrm{mmol}, 63 \%\right.$ yield overall) as colorless oil. 14: $\mathrm{R}_{\mathrm{f}}=0.40\left(\mathrm{SiO}_{2}\right.$, hexanes/EtOAc 5:1, v/v); FT-IR (film) $v_{\max }=3293,2931,2859,1495,1454,1361,1091,735 \mathrm{~cm}^{-1} ;{ }^{1} \mathrm{H}$ NMR (mixture of isomers, $\left.600 \mathrm{MHz}, \mathrm{CDCl}_{3}\right) \delta=7.36-7.33(\mathrm{~m}, 4 \mathrm{H}), 7.31-7.26(\mathrm{~m}, 1 \mathrm{H}), 4.51(\mathrm{~s}, 2 \mathrm{H}), 4.26(\mathrm{dt}, J=6.2,2.8 \mathrm{~Hz}, 0.53 \mathrm{H})$, $4.16(\mathrm{dt}, J=6.2,3.1 \mathrm{~Hz}, 0.45 \mathrm{H}), 3.81(\mathrm{dq}, J=7.5,4.9 \mathrm{~Hz}, 0.51 \mathrm{H}), 3.73(\mathrm{tt}, J=6.6,4.9 \mathrm{~Hz}, 0.46 \mathrm{H}), 3.58(\mathrm{dd}$, $J=10.3,4.3 \mathrm{~Hz}, 0.48 \mathrm{H}), 3.51(\mathrm{td}, J=5.9,3.2 \mathrm{~Hz}, 2 \mathrm{H}), 3.46-3.36(\mathrm{~m}, 1.6 \mathrm{H}), 2.47-2.43(\mathrm{~m}, 0.42 \mathrm{H}), 2.41$ $(\mathrm{dd}, J=2.1,0.8 \mathrm{~Hz}, 0.49 \mathrm{H}), 1.88-1.77(\mathrm{~m}, 4.0 \mathrm{H}), 1.72-1.54(\mathrm{~m}, 2.0 \mathrm{H}), 1.46-1.23(\mathrm{~m}, 4 \mathrm{H}), 0.91(\mathrm{t}$, $J=6.9 \mathrm{~Hz}, 3 \mathrm{H}$ ) ppm; ${ }^{13} \mathrm{C} \mathrm{NMR}$ (mixture of isomers, $151 \mathrm{MHz}, \mathrm{CDCl}_{3}$ ) $\delta=138.7,138.6,128.5,127.8,127.7$, $127.67,127.65,83.4,83.0,78.0,76.2,74.0,73.9,73.0,70.0,69.9,68.8,67.7,35.4,34.9,33.5,33.0,32.9$, 32.3, 27.4, 27.1, 25.63, 25.62, 22.8, 22.7, 14.14, 14.12 ppm; HRMS (ESI-TOF) calcd for $\mathrm{C}_{19} \mathrm{H}_{27} \mathrm{BrO}_{2} \mathrm{Na}^{+}$ $[\mathrm{M}+\mathrm{Na}]^{+}$389.1087; Found 389.1089. 
2-[3-(Benzyloxy)propyl]-5-butyl-3-methylidenetetrahydrofuran (15): To a stirred solution of bromide

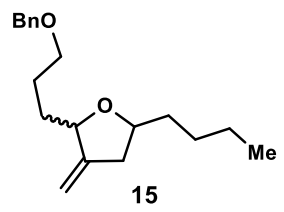
$14(0.10 \mathrm{~g}, 0.27 \mathrm{mmol}, 1.0$ equiv $)$ in toluene $(20 \mathrm{~mL})$ was added $n-\mathrm{Bu}_{3} \mathrm{SnH}(0.11 \mathrm{~mL}$, $0.41 \mathrm{mmol}, 1.5$ equiv) followed by AIBN $(4.5 \mathrm{mg}, 0.027 \mathrm{mmol}, 0.1$ equiv) in one portion, and the resulting mixture was heated to $90^{\circ} \mathrm{C}$. After $0.5 \mathrm{~h}$, the reaction mixture was allowed to cool to $23^{\circ} \mathrm{C}$ and the solvent was removed under reduced pressure. The obtained residue was purified by flash column chromatography $\left(\mathrm{SiO}_{2}, 2 \rightarrow 10 \%\right.$ EtOAc in hexanes) to afford a mixture of diastereomers of tetrahydrofuran derivative $15(3: 2 d r, 64 \mathrm{mg}, 0.22 \mathrm{mmol}, 82 \%$ yield $)$ as a colorless foam. 15: $\mathrm{R}_{\mathrm{f}}=0.20\left(\mathrm{SiO}_{2}\right.$, hexanes/EtOAc 20:1, v/v); FT-IR (film) $v_{\max }=3030,2929,2857,1496,1454,1361,1204$, $1098,883,734 \mathrm{~cm}^{-1} ;{ }^{1} \mathrm{H}$ NMR (mixture of isomers, $\left.600 \mathrm{MHz}, \mathrm{CDCl}_{3}\right) \delta=7.34(\mathrm{~d}, J=4.4 \mathrm{~Hz}, 4 \mathrm{H}), 7.29$ $7.26(\mathrm{~m}, 1 \mathrm{H}), 4.97(\mathrm{q}, J=2.2 \mathrm{~Hz}, 0.6 \mathrm{H}), 4.94(\mathrm{td}, J=2.5,1.6 \mathrm{~Hz}, 0.4 \mathrm{H}), 4.84(\mathrm{q}, J=2.2 \mathrm{~Hz}, 0.6 \mathrm{H}), 4.81(\mathrm{dt}$, $J=3.4,1.9 \mathrm{~Hz}, 0.4 \mathrm{H}), 4.51(\mathrm{~s}, 2 \mathrm{H}), 4.39(\mathrm{dq}, J=6.2,2.2 \mathrm{~Hz}, 0.6 \mathrm{H}), 4.30-4.24(\mathrm{~m}, 0.4 \mathrm{H}), 3.98(\mathrm{p}, J=6.6 \mathrm{~Hz}$, $0.6 \mathrm{H}), 3.80$ (dtd, $J=9.9,6.4,5.5 \mathrm{~Hz}, 0.4 \mathrm{H}), 3.56-3.47$ (m, 2H), 2.65 (ddq, $J=15.5,6.1,2.0 \mathrm{~Hz}, 0.6 \mathrm{H}), 2.59$ (ddq, $J=15.4,5.4,1.5 \mathrm{~Hz}, 0.4 \mathrm{H}), 2.24$ (ddtd, $J=15.5,7.0,2.4,0.9 \mathrm{~Hz}, 0.6 \mathrm{H}), 2.16$ (ddq, $J=15.2,10.0$, $2.6 \mathrm{~Hz}, 0.4 \mathrm{H}), 1.85-1.64(\mathrm{~m}, 3.6 \mathrm{H}), 1.62-1.54(\mathrm{~m}, 1.4 \mathrm{H}), 1.54-1.44(\mathrm{~m}, 0.4 \mathrm{H}), 1.45-1.37(\mathrm{~m}, 1 \mathrm{H}), 1.37-$ $1.23(\mathrm{~m}, 3.6 \mathrm{H}), 0.90(\mathrm{td}, J=7.1,2.1 \mathrm{~Hz}, 3 \mathrm{H}) \mathrm{ppm} ;{ }^{13} \mathrm{C}$ NMR (mixture of isomers, $151 \mathrm{MHz}, \mathrm{CDCl}_{3}$ ) $\delta=152.3,152.1,138.8,138.8,128.5,127.8,127.60,127.59,104.7,104.2,80.5,79.6,78.5,77.4,73.0,72.9$, $70.6,70.5,39.7,39.0,35.3,35.1,32.1,31.9,28.3,28.3,26.0,25.6,23.0,22.9,14.21,14.19$ ppm; HRMS (ESI-TOF) calcd for $\mathrm{C}_{19} \mathrm{H}_{28} \mathrm{O}_{2} \mathrm{Na}^{+}[\mathrm{M}+\mathrm{Na}]^{+}$311.1982; Found 311.1981.

(6S,8S)-8-Ethynyl-13,13-dimethyl-1,12,12-triphenyl-6-(prop-2-en-1-yl)-2,7,11-trioxa-12-silatetradecane (18a) and (6S,8R)-8-ethynyl-13,13-dimethyl-1,12,12-triphenyl-6-(prop-2-en-1-yl)-2,7,11trioxa-12-silatetradecane (18b): To a stirred solution of alkyne $\mathbf{1 7}^{3}(3.00 \mathrm{~g}, 8.86 \mathrm{mmol}, 1.0 \mathrm{equiv})$ in

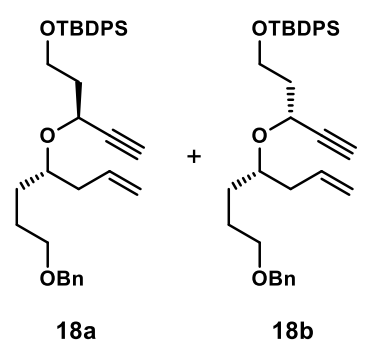
$\mathrm{CH}_{2} \mathrm{Cl}_{2}(10 \mathrm{~mL})$ at $25^{\circ} \mathrm{C}$ was added $\mathrm{Co}_{2}(\mathrm{CO})_{8}(3.63 \mathrm{~g}, 10.6 \mathrm{mmol}, 1.2$ equiv) in one portion. After $20 \mathrm{~min}$, a solution of olefin $16^{4}(3.90 \mathrm{~g}, 17.7 \mathrm{mmol}, 2.0$ equiv) in $\mathrm{CH}_{2} \mathrm{Cl}_{2}(8 \mathrm{~mL})$ was added. The reaction mixture was cooled to $0{ }^{\circ} \mathrm{C}$, and $\mathrm{BF}_{3} \cdot \mathrm{Et}_{2} \mathrm{O}(2.19 \mathrm{~mL}, 17.7 \mathrm{mmol}, 2.0$ equiv $)$ was added dropwise. After $0.5 \mathrm{~h}$, the reaction mixture was carefully quenched by the addition of saturated aqueous 
$\mathrm{NaHCO}_{3}$ solution $(50 \mathrm{~mL})$, and allowed to warm to $23^{\circ} \mathrm{C}$. The aqueous layer was extracted with $\mathrm{CH}_{2} \mathrm{Cl}_{2}$ $(3 \times 20 \mathrm{~mL})$ and the combined organic layers were dried over anhydrous $\mathrm{Na}_{2} \mathrm{SO}_{4}$, filtered, and concentrated under reduced pressure.

To a stirred solution of the so-obtained crude residue in acetone $(30 \mathrm{~mL})$ was added portion wise diammonium cerium(IV) nitrate (CAN) $\left(24.3 \mathrm{~g}, 44.3 \mathrm{mmol}, 5.0\right.$ equiv) at $0{ }^{\circ} \mathrm{C}$. The resulting mixture was allowed to warm to $23^{\circ} \mathrm{C}$ and stirred for $1 \mathrm{~h}$ before it was quenched by the addition of water $(60 \mathrm{~mL})$. The aqueous layer was extracted with EtOAc $(3 \times 50 \mathrm{~mL})$ and the combined organic layers were dried over anhydrous $\mathrm{Na}_{2} \mathrm{SO}_{4}$, filtered, and concentrated under reduced pressure. The obtained residue was purified by flash column chromatography $\left(\mathrm{SiO}_{2}, 1 \rightarrow 5 \%\right.$ ethyl acetate in hexanes) to afford cis-isomer $\mathbf{1 8 b}(2.01 \mathrm{~g}$, $3.72 \mathrm{mmol}, 42 \%$ yield overall) and trans-isomer 18a (1.68 g, $3.10 \mathrm{mmol}, 35 \%$ yield overall) as colorless oils. 18b (cis-isomer): $\mathrm{R}_{\mathrm{f}}=0.50\left(\mathrm{SiO}_{2}\right.$, hexanes/EtOAc 20:1, v/v); $[\alpha]_{\mathrm{D}}^{23}=+6.65\left(c=2.0, \mathrm{CH}_{2} \mathrm{Cl}_{2}\right)$; FT-IR (film) $v_{\max }=3289,2999,2857,2150,1472,1427,1173,1093,700 \mathrm{~cm}^{-1} ;{ }^{1} \mathrm{H} \mathrm{NMR}\left(600 \mathrm{MHz}, \mathrm{CDCl}_{3}\right)$ $\delta=7.67(\mathrm{dt}, J=8.0,1.6 \mathrm{~Hz}, 4 \mathrm{H}), 7.41(\mathrm{tdd}, J=6.1,3.8,2.2 \mathrm{~Hz}, 2 \mathrm{H}), 7.37-7.32(\mathrm{~m}, 4 \mathrm{H}), 7.33(\mathrm{~d}, J=6.3 \mathrm{~Hz}$, 4H), $7.28(\mathrm{td}, J=6.0,2.6 \mathrm{~Hz}, 1 \mathrm{H}), 5.87(\mathrm{ddt}, J=17.2,10.2,7.0 \mathrm{~Hz}, 1 \mathrm{H}), 5.14-5.00(\mathrm{~m}, 2 \mathrm{H}), 4.48(\mathrm{~d}$, $J=2.6 \mathrm{~Hz}, 2 \mathrm{H}), 4.45-4.41(\mathrm{~m}, 1 \mathrm{H}), 3.88-3.74(\mathrm{~m}, 2 \mathrm{H}), 3.66(\mathrm{p}, J=5.8 \mathrm{~Hz}, 1 \mathrm{H}), 3.44(\mathrm{td}, J=6.4,2.2 \mathrm{~Hz}$, 2H), 2.44-2.30 (m, 3 H), 2.00 (dtd, $J=13.4,7.0,5.2 \mathrm{~Hz}, 1 \mathrm{H}), 1.90(\mathrm{dtd}, J=13.2,6.7,5.4 \mathrm{~Hz}, 1 \mathrm{H}), 1.70$ (ddt, $J=11.9,8.1,6.1 \mathrm{~Hz}, 1 \mathrm{H}), 1.65-1.52(\mathrm{~m}, 3 \mathrm{H}), 1.05(\mathrm{~s}, 9 \mathrm{H}) \mathrm{ppm} ;{ }^{13} \mathrm{C} \mathrm{NMR}\left(151 \mathrm{MHz}, \mathrm{CDCl}_{3}\right) \delta=138.7$, 135.70, 135.66, 135.2, 133.9, 133.8, 129.8, 128.5, 127.79, 127.77, 127.75, 127.6, 116.9, 84.1, 78.0, 73.2, 73.0, 70.6, 64.8, 60.0, 39.4, 39.1, 30.0, 27.0, 25.5, 19.3 ppm; HRMS (ESI-TOF) calcd for $\mathrm{C}_{35} \mathrm{H}_{44} \mathrm{O}_{3} \mathrm{SiNa}^{+}$ $[\mathrm{M}+\mathrm{Na}]^{+}$563.2952; Found 563.2957. 18a (trans-isomer): $\mathrm{R}_{\mathrm{f}}=0.48\left(\mathrm{SiO}_{2}\right.$, hexanes/EtOAc 20:1, v/v); $[\alpha]_{\mathrm{D}}^{23}=-26.6\left(c=1.5, \mathrm{CH}_{2} \mathrm{Cl}_{2}\right)$; FT-IR (film) $v_{\max }=3303,3030,2857,2188,1640,1589,1428,1361,1104$, 1089, $735 \mathrm{~cm}^{-1} ;{ }^{1} \mathrm{H}$ NMR $\left(600 \mathrm{MHz}, \mathrm{CDCl}_{3}\right) \delta=7.69-7.64(\mathrm{~m}, 4 \mathrm{H}), 7.45-7.40(\mathrm{~m}, 2 \mathrm{H}), 7.40-7.36(\mathrm{~m}, 4 \mathrm{H})$, $7.34(\mathrm{~d}, J=4.4 \mathrm{~Hz}, 4 \mathrm{H}), 7.30-7.26(\mathrm{~m}, 1 \mathrm{H}), 5.80(\mathrm{ddt}, J=17.3,10.2,7.2 \mathrm{~Hz}, 1 \mathrm{H}), 5.13-4.97(\mathrm{~m}, 2 \mathrm{H}), 4.52-$ $4.46(\mathrm{~m}, 3 \mathrm{H}), 3.85-3.76(\mathrm{~m}, 2 \mathrm{H}), 3.69$ (ddt, $J=8.6,6.4,4.6 \mathrm{~Hz}, 1 \mathrm{H}), 3.55-3.42(\mathrm{~m}, 2 \mathrm{H}), 2.31(\mathrm{~d}, J=2.0 \mathrm{~Hz}$, $1 \mathrm{H}), 2.30-2.23$ (m, 2H), 1.98 (dddd, $J=13.7,7.6,6.3,5.0 \mathrm{~Hz}, 1 \mathrm{H}), 1.90$ (dddd, $J=13.4,7.0,6.1,5.3 \mathrm{~Hz}$, $1 \mathrm{H}), 1.80$ (dddd, $J=13.1,9.7,6.6,5.0 \mathrm{~Hz}, 1 \mathrm{H}), 1.73-1.59$ (m, 2H), 1.55-1.47 (m, $1 \mathrm{H}), 1.05$ (s, $9 \mathrm{H}) \mathrm{ppm}$; ${ }^{13} \mathrm{C}$ NMR $\left(151 \mathrm{MHz}, \mathrm{CDCl}_{3}\right) \delta=138.9,135.7,135.6,134.7,133.9,133.8,129.7,128.5,127.8,127.7,127.5$, 
$117.4,84.0,76.8,73.1,72.9,70.5,64.2,60.0,39.3,38.1,31.0,27.0,25.9,19.3$ ppm; HRMS (ESI-TOF) calcd for $\mathrm{C}_{35} \mathrm{H}_{44} \mathrm{O}_{3} \mathrm{SiNa}^{+}[\mathrm{M}+\mathrm{Na}]^{+}$563.2952; Found 563.2952.

\section{(2R,4R,6S)-6-[3-(Benzyloxy)propyl]-2-(2-\{[tert-butyl(diphenyl)silyl]oxy\}ethyl)-4-methyldihydro-2H-} pyran-3(4H)-one (20a): To a stirred solution of a mixture of alkynes $18 \mathbf{b}$ and $\mathbf{1 8 a}$ [2.00 $\mathrm{g}(\mathbf{1 8 b}: \mathbf{1 8 a}=1.2: 1)$,

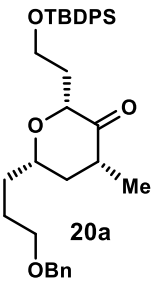
$3.70 \mathrm{mmol}, 1.0$ equiv] in $\mathrm{Et}_{2} \mathrm{O}(600 \mathrm{~mL})$ at $-78^{\circ} \mathrm{C}$ was added $\mathrm{Ti}(\mathrm{O} i-\mathrm{Pr})_{4}(1.64 \mathrm{~g}, 5.55 \mathrm{mmol}$, 1.5 equiv), followed by diisoproyl magnesium chloride ( $2 \mathrm{M}$ solution in THF, $5.55 \mathrm{~mL}$, $11.1 \mathrm{mmol}, 3.0$ equiv). After $5 \mathrm{~min}$ of stirring, the reaction mixture was allowed to warm to $0{ }^{\circ} \mathrm{C}$ over $1 \mathrm{~h}$ and then quenched by the addition of $0.5 \mathrm{M}$ aqueous $\mathrm{HCl}(100 \mathrm{~mL})$. The layers were separated, the aqueous layer was extracted with $\mathrm{Et}_{2} \mathrm{O}(3 \times 100 \mathrm{~mL})$ and the combined organic layers were washed with sat. $\mathrm{NaHCO}_{3}$, dried over anhydrous $\mathrm{Na}_{2} \mathrm{SO}_{4}$, filtered, and concentrated under reduced pressure. The obtained residue was passed through pad of silica gel to obtain a mixture of compounds 19ac $(1.80 \mathrm{~g}, 3.33 \mathrm{mmol}, 90 \%$ yield $)$ that was used for the next step without further purification.

A stirred solution of the crude residue obtained above in $\mathrm{CH}_{2} \mathrm{Cl}_{2}(80 \mathrm{~mL})$ was cooled to $-78^{\circ} \mathrm{C}$. Freshly generated ozone was bubbled through this solution until the color of the mixture changed to light blue (ca. $15 \mathrm{~min})$. The reaction mixture was quenched by the addition of dimethyl sulfide $(2.72 \mathrm{~mL}, 37.0 \mathrm{mmol}$, 10.0 equiv), and allowed to warm to $23^{\circ} \mathrm{C}$. After stirring for $10 \mathrm{~h}$, the solvent was removed under reduced pressure, and the obtained residue was purified by flash column chromatography $\left(\mathrm{SiO}_{2}, 5 \rightarrow 20 \%\right.$ ethyl acetate in hexanes) to afford a mixture of three diastereomers $\mathbf{2 0}(1.57 \mathrm{~g}, 2.89 \mathrm{mmol}, 87 \%$ yield overall $)$ as a colorless oil. The mixture of diastereomers was subjected to isomerization under basic conditions without further purification.

To a stirred solution of the above mixture of diastereomers $\mathbf{2 0}(1.50 \mathrm{~g}, 2.75 \mathrm{mmol}, 1.0$ equiv) in $\mathrm{MeOH}$ (30 mL) was added $\mathrm{K}_{2} \mathrm{CO}_{3}\left(571 \mathrm{mg}, 4.13 \mathrm{mmol}, 1.5\right.$ equiv) and the resulting mixture was heated to $70^{\circ} \mathrm{C}$. After $4 \mathrm{~h}$, the reaction mixture was allowed to cool to $23^{\circ} \mathrm{C}$ and quenched by the addition of saturated aqueous $\mathrm{NH}_{4} \mathrm{Cl}$ solution $(40 \mathrm{~mL})$. The aqueous layer was extracted with EtOAc $(3 \times 50 \mathrm{~mL})$ and the combined organic layers were dried over anhydrous $\mathrm{Na}_{2} \mathrm{SO}_{4}$, filtered, and concentrated under reduced pressure. The obtained residue was purified by flash column chromatography $\left(\mathrm{SiO}_{2}, 5 \rightarrow 20 \%\right.$ ethyl acetate 
in hexanes) to afford the required isomer $\mathbf{2 0 a}(899 \mathrm{mg}, 1.65 \mathrm{mmol}, 60 \%$ yield) as a colorless oil. 20a: $\mathrm{R}_{\mathrm{f}}=0.45\left(\mathrm{SiO}_{2}\right.$, hexanes $\left./ \mathrm{Et}_{2} \mathrm{O} 5: 1, v / v\right) ;[\alpha]_{\mathrm{D}}^{23}=+4.75\left(c=2.8, \mathrm{CH}_{2} \mathrm{Cl}_{2}\right)$; FT-IR (film) $v_{\max }=3070,2957,2856$, $1721,1472,1427,1361,1260,1107,1091,822,699 \mathrm{~cm}^{-1} ;{ }^{1} \mathrm{H}$ NMR $\left(600 \mathrm{MHz}, \mathrm{CDCl}_{3}\right) \delta=7.75-7.73(\mathrm{~m}$, 1H), 7.69-7.64 (m, 4H), 7.46-7.34 (m, 9H), 7.32-7.28 (m, 1 H), 4.51 (AB quart, $J=11.2 \mathrm{~Hz}, 2 \mathrm{H}), 4.14-$ 4.04 (m, $1 \mathrm{H}), 3.85$ (tdd, $J=9.3,4.7,1.5 \mathrm{~Hz}, 1 \mathrm{H}), 3.82-3.75(\mathrm{~m}, 2 \mathrm{H}), 3.56-3.43(\mathrm{~m}, 2 \mathrm{H}), 2.55$ (dp, $J=12.9$, $6.5 \mathrm{~Hz}, 1 \mathrm{H}), 2.27-2.20(\mathrm{~m}, 1 \mathrm{H}), 2.19-2.15(\mathrm{~m}, 1 \mathrm{H}), 1.79(\mathrm{ddt}, J=12.6,9.8,5.9 \mathrm{~Hz}, 1 \mathrm{H}), 1.73-1.65(\mathrm{~m}$, 2H), 1.64-1.52 (m, 3H), $1.09(\mathrm{~d}, J=6.5 \mathrm{~Hz}, 3 \mathrm{H}), 1.06(\mathrm{~s}, 9 \mathrm{H}) \mathrm{ppm} ;{ }^{13} \mathrm{C} \mathrm{NMR}\left(151 \mathrm{MHz}, \mathrm{CDCl}_{3}\right) \delta=209.6$, $138.7,135.6,135.3,134.9,129.7,128.5,127.8,127.78,127.75,78.4,76.8,73.0,70.2,59.9,42.8,42.6$, 32.24, 32.17, 27.0, 26.7, 19.4, 14.5 ppm; HRMS (ESI-TOF) calcd for $\mathrm{C}_{34} \mathrm{H}_{44} \mathrm{O}_{4} \mathrm{SiNa}^{+}[\mathrm{M}+\mathrm{Na}]^{+}$567.2901; Found 567.2900.

\section{(2-\{(2R,4R,6S)-6-[3-(Benzyloxy)propyl]-4-methyl-3-methylidenetetrahydro-2H-pyran-2-yl\}ethoxy)-}

(tert-butyl)diphenylsilane (19a): To a stirred solution of methyl triphenyl phosphonium bromide $(2.62 \mathrm{~g}$,

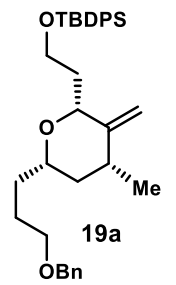

$7.34 \mathrm{mmol}, 5.0$ equiv) in THF $(40 \mathrm{~mL})$ at $-78^{\circ} \mathrm{C}$ was added dropwise $n$-butyl lithium $(1.6 \mathrm{M}$ in hexanes, $4.13 \mathrm{~mL}, 6.62 \mathrm{mmol}, 4.5$ equiv), and the reaction mixture was warmed to $0{ }^{\circ} \mathrm{C}$. After $0.5 \mathrm{~h}$, a solution of ketone 20a $(800 \mathrm{mg}, 1.47 \mathrm{mmol}, 1.0$ equiv) in THF $(15 \mathrm{~mL})$ was added to the preformed ylide solution and the resulting mixture was allowed to warm to $23^{\circ} \mathrm{C}$.

After $1 \mathrm{~h}$, the reaction mixture was carefully quenched by the addition of saturated aqueous $\mathrm{NH}_{4} \mathrm{Cl}$ solution $(80 \mathrm{~mL})$. The aqueous layer was extracted with EtOAc $(3 \times 30 \mathrm{~mL})$ and the combined organic layers were dried over anhydrous $\mathrm{Na}_{2} \mathrm{SO}_{4}$, filtered, and concentrated under reduced pressure. The obtained residue was purified by flash column chromatography $\left(\mathrm{SiO}_{2}, 2 \rightarrow 10 \%\right.$ EtOAc in hexanes) to afford pure olefin 19a (734 mg, $1.35 \mathrm{mmol}, 92 \%$ yield) as a colorless foam. 19a: $\mathrm{R}_{\mathrm{f}}=0.55\left(\mathrm{SiO}_{2}\right.$, hexanes/EtOAc 5:1, v/v); $[\alpha]_{\mathrm{D}}^{23}=+6.60\left(c=2.0, \mathrm{CH}_{2} \mathrm{Cl}_{2}\right)$; FT-IR (film) $v_{\max }=2956,2929,2855,1649,1472,1361,1261,1105,1084$, 822, $735 \mathrm{~cm}^{-1} ;{ }^{1} \mathrm{H}$ NMR $\left(600 \mathrm{MHz}, \mathrm{CDCl}_{3}\right) \delta=7.72-7.65(\mathrm{~m}, 4 \mathrm{H}), 7.43-7.35(\mathrm{~m}, 6 \mathrm{H}), 7.35-7.32(\mathrm{~m}, 4 \mathrm{H})$, $7.28(\mathrm{tt}, J=6.9,2.4 \mathrm{~Hz}, 1 \mathrm{H}), 4.85(\mathrm{~d}, J=1.8 \mathrm{~Hz}, 1 \mathrm{H}), 4.79(\mathrm{~d}, J=1.8 \mathrm{~Hz}, 1 \mathrm{H}), 4.48$ (AB quart, $J=11.5 \mathrm{~Hz}$, 2H), 3.96-3.89 (m, 2 H), $3.83(\mathrm{ddd}, J=10.1,6.5,4.0 \mathrm{~Hz}, 1 \mathrm{H}), 3.55-3.40(\mathrm{~m}, 3 \mathrm{H}), 2.28(\mathrm{dqd}, J=12.2,6.2$, $3.1 \mathrm{~Hz}, 1 \mathrm{H}), 2.13-2.04(\mathrm{~m}, 1 \mathrm{H}), 1.89-1.68(\mathrm{~m}, 3 \mathrm{H}), 1.67-1.59(\mathrm{~m}, 1 \mathrm{H}), 1.55-1.48(\mathrm{~m}, 2 \mathrm{H}), 1.10-1.08(\mathrm{~m}$, 
4H), $1.06(\mathrm{~s}, 9 \mathrm{H}) \mathrm{ppm} ;{ }^{13} \mathrm{C} \mathrm{NMR}\left(151 \mathrm{MHz}, \mathrm{CDCl}_{3}\right) \delta=151.9,138.8,135.7,135.6,134.2,129.6,128.4$, $127.8,127.7,127.6,104.3,77.4,74.9,72.9,70.5,60.8,43.4,36.0,34.7,32.8,27.0,26.2,19.4,18.2$ ppm; HRMS (ESI-TOF) calcd for $\mathrm{C}_{35} \mathrm{H}_{46} \mathrm{O}_{3} \mathrm{SiNa}^{+}[\mathrm{M}+\mathrm{Na}]^{+}$565.3108; Found 565.3111.

\section{2-\{(2R,4R,6S)-6-[3-(Benzyloxy)propyl]-4-methyl-3-methylidenetetrahydro-2H-pyran-2-yl\}ethanol}

(S1): To a stirred solution of olefin derivative 19a $\left(500 \mathrm{mg}, 0.921 \mathrm{mmol}, 1.0\right.$ equiv) in $\mathrm{THF}(20 \mathrm{~mL})$ at $0{ }^{\circ} \mathrm{C}$

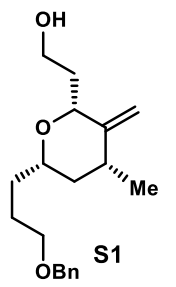
was added dropwise tetra- $n$-butylammonium fluoride $(1 \mathrm{M}$ in THF, $0.920 \mathrm{~mL}, 0.920 \mathrm{mmol}$, 1.0 equiv), and the reaction mixture was allowed to warm to $23^{\circ} \mathrm{C}$. After $2.5 \mathrm{~h}$, the reaction

mixture was quenched by the addition of saturated aqueous $\mathrm{NH}_{4} \mathrm{Cl}$ solution $(15 \mathrm{~mL})$. The aqueous layer was extracted with EtOAc $(3 \times 15 \mathrm{~mL})$ and the combined organic layers were dried over anhydrous $\mathrm{Na}_{2} \mathrm{SO}_{4}$, filtered, and concentrated under reduced pressure. The obtained residue was purified by flash column chromatography $\left(\mathrm{SiO}_{2}, 10 \rightarrow 40 \%\right.$ EtOAc in hexanes) to afford pure alcohol $\mathbf{S 1}$ (264 mg, $0.866 \mathrm{mmol}, 94 \%$ yield) as a colorless oil. $\mathbf{S 1}: \mathrm{R}_{\mathrm{f}}=0.40\left(\mathrm{SiO}_{2}\right.$, hexanes/EtOAc 3:2, v/v); $[\alpha]_{\mathrm{D}}^{23}=+8.10\left(c=2.0, \mathrm{CH}_{2} \mathrm{Cl}_{2}\right)$; FT-IR (film) $v_{\max }=3431,2954,2850,1650,1454,1364,1312,1091,1058$, 903, $736 \mathrm{~cm}^{-1} ;{ }^{1} \mathrm{H}$ NMR $\left(600 \mathrm{MHz}, \mathrm{CDCl}_{3}\right) \delta=7.36-7.32(\mathrm{~m}, 4 \mathrm{H}), 7.30-7.26(\mathrm{~m}, 1 \mathrm{H}), 4.87(\mathrm{~d}, J=1.6 \mathrm{~Hz}$, $1 \mathrm{H}), 4.82(\mathrm{~d}, J=1.6 \mathrm{~Hz}, 1 \mathrm{H}), 4.49(\mathrm{~s}, 2 \mathrm{H}), 3.95-3.89(\mathrm{~m}, 1 \mathrm{H}), 3.84(\mathrm{dd}, J=6.0,4.9 \mathrm{~Hz}, 2 \mathrm{H}), 3.66-3.57(\mathrm{~m}$, 1 H), 3.53-3.41 (m, 2 H), 2.31-2.23 (m, $1 \mathrm{H}), 2.02-1.91(\mathrm{~m}, 2 \mathrm{H}), 1.81-1.61(\mathrm{~m}, 3 \mathrm{H}), 1.56(\mathrm{td}, J=7.6,6.1 \mathrm{~Hz}$, 2H), 1.13-1.06 (m, 4H) ppm; ${ }^{13} \mathrm{C}$ NMR $\left(151 \mathrm{MHz}, \mathrm{CDCl}_{3}\right) \delta=150.9,138.7,128.5,127.8,127.6,105.0$, 79.4, 77.7, 73.0, 70.3, 61.8, 42.6, 35.6, 33.5, 32.8, 26.1, $18.1 \mathrm{ppm}$; HRMS (ESI-TOF) calcd for $\mathrm{C}_{19} \mathrm{H}_{28} \mathrm{O}_{3} \mathrm{Na}^{+}$ $[\mathrm{M}+\mathrm{Na}]^{+}$327.1931; Found 327.1930.

$\{(2 R, 4 R, 6 S)-6-[3-(B e n z y l o x y)$ propyl]-4-methyl-3-methylidenetetrahydro-2H-pyran-2-yl\}acetaldehyde (21): To a stirred solution of alcohol $\mathbf{S 1}\left(200 \mathrm{mg}, 0.657 \mathrm{mmol}, 1.0\right.$ equiv) in $\mathrm{CH}_{2} \mathrm{Cl}_{2}(10 \mathrm{~mL})$ at<smiles>C=C1[C@H](C)CC(CCCO)O[C@@H]1CC=O</smiles>
$23^{\circ} \mathrm{C}$ was added DMP $(418 \mathrm{mg}, 0.985 \mathrm{mmol}, 1.5$ equiv) portion wise. After $0.5 \mathrm{~h}$, the reaction mixture was quenched by the addition of saturated aqueous $\mathrm{Na}_{2} \mathrm{~S}_{2} \mathrm{O}_{3}$ solution $(20 \mathrm{~mL})$ and the obtained suspension was further stirred for $2 \mathrm{~h}$. The aqueous layer was extracted with $\mathrm{CH}_{2} \mathrm{Cl}_{2}$ $(3 \times 10 \mathrm{~mL})$ and the combined organic layers were washed with saturated aqueous $\mathrm{NaHCO}_{3}$ 
solution $(10 \mathrm{~mL})$, dried over anhydrous $\mathrm{Na}_{2} \mathrm{SO}_{4}$, filtered, and concentrated under reduced pressure. The obtained residue was purified by flash column chromatography $\left(\mathrm{SiO}_{2}, 10 \rightarrow 30 \%\right.$ EtOAc in hexanes) to afford pure aldehyde $21(179 \mathrm{mg}, 0.591 \mathrm{mmol}, 90 \%$ yield $)$ as a colorless foam. 21: $\mathrm{R}_{\mathrm{f}}=0.80\left(\mathrm{SiO}_{2}\right.$, hexanes/EtOAc 3:2,v/v); $[\alpha]_{\mathrm{D}}^{23}=+17.0\left(c=2.0, \mathrm{CH}_{2} \mathrm{Cl}_{2}\right)$; FT-IR (film) $v_{\max }=3004,2848,2725,1725,1650$, 1454, 1363, 1087, 1028, 904, $735 \mathrm{~cm}^{-1} ;{ }^{1} \mathrm{H} \mathrm{NMR}\left(600 \mathrm{MHz}, \mathrm{CDCl}_{3}\right) \delta=9.81(\mathrm{dd}, J=2.7,2.0 \mathrm{~Hz}, 1 \mathrm{H})$, 7.36-7.32 (m, 4H), 7.30-7.26 (m, 1 H), $4.84(\mathrm{dd}, J=1.9,0.6 \mathrm{~Hz}, 1 \mathrm{H}), 4.75(\mathrm{~d}, J=1.9 \mathrm{~Hz}, 1 \mathrm{H}), 4.49(\mathrm{~s}, 2 \mathrm{H})$, 4.29-4.22 (m, $1 \mathrm{H}), 3.62-3.54(\mathrm{~m}, 1 \mathrm{H}), 3.47$ (ddt, $J=26.7,9.3,6.5 \mathrm{~Hz}, 2 \mathrm{H}), 2.79-2.65$ (m, 2 H), 2.36-2.28 (m, 1 H), 1.84-1.68 (m, 2H), 1.67-1.59 (m, 1 H), 1.57-1.47 (m, 2 H), 1.14-1.07 (m, 4 H) ppm; ${ }^{13} \mathrm{C}$ NMR $\left(151 \mathrm{MHz}, \mathrm{CDCl}_{3}\right) \delta=201.9,150.2,138.7,128.4,127.8,127.6,105.4,77.7,74.4,72.9,70.3,45.7,42.6$, 35.6, 32.5, 26.0, 18.0 ppm; HRMS (ESI-TOF) calcd for $\mathrm{C}_{19} \mathrm{H}_{26} \mathrm{O}_{3} \mathrm{Na}^{+}[\mathrm{M}+\mathrm{Na}]^{+}$325.1774; Found 325.1771.

\section{1-\{(2R,4R,6S)-6-[3-(Benzyloxy)propyl]-4-methyl-3-methylidenetetrahydro-2H-pyran-2-yl\}but-3-yn-}

2-ol (22): To a stirred solution of aldehyde $21\left(600 \mathrm{mg}, 1.98 \mathrm{mmol}, 1.0\right.$ equiv) in THF $(15 \mathrm{~mL})$ at $-78^{\circ} \mathrm{C}$

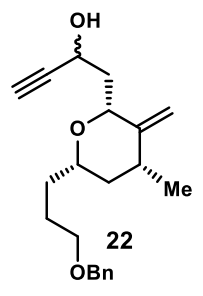
was added ethynylmagnesium bromide $(0.5 \mathrm{M}$ in THF, $9.92 \mathrm{~mL}, 4.96 \mathrm{mmol}, 2.5$ equiv), and the reaction mixture was warmed to $-10^{\circ} \mathrm{C}$. After $15 \mathrm{~min}$, the reaction mixture was quenched by the addition of saturated aqueous $\mathrm{NH}_{4} \mathrm{Cl}$ solution $(30 \mathrm{~mL})$. The aqueous layer was extracted with EtOAc $(3 \times 20 \mathrm{~mL})$ and the combined organic layers were dried over anhydrous $\mathrm{Na}_{2} \mathrm{SO}_{4}$, filtered, and concentrated under reduced pressure. The obtained residue was purified by flash column chromatography $\left(\mathrm{SiO}_{2}, 5 \rightarrow 20 \%\right.$ EtOAc in hexanes) to afford alcohol 22 (mixture of diastereomers, $1.5: 1 \mathrm{dr}, 520 \mathrm{mg}, 1.58 \mathrm{mmol}, 80 \%$ yield $)$ as a colorless foam. 22: $\mathrm{R}_{\mathrm{f}}=0.35\left(\mathrm{SiO}_{2}\right.$, hexanes/EtOAc 5:1, v/v); $[\alpha]_{\mathrm{D}}^{23}=+21.3\left(c=3.0, \mathrm{CH}_{2} \mathrm{Cl}_{2}\right)$; FT-IR (film) $v_{\max }=3415,3297,2956,2850,1650$, 1454, 1365, 1312, 1206, 1089, 1062, 903, $697 \mathrm{~cm}^{-1} ;{ }^{1} \mathrm{H}$ NMR $\left(600 \mathrm{MHz}, \mathrm{CDCl}_{3}\right) \delta=7.36-7.31(\mathrm{~m}, 4 \mathrm{H})$, 7.29-7.26 (m, $1 \mathrm{H}), 4.87(\mathrm{~d}, J=1.5 \mathrm{~Hz}, 0.6 \mathrm{H}), 4.84(\mathrm{~d}, J=1.6 \mathrm{~Hz}, 0.4 \mathrm{H}), 4.83(\mathrm{~d}, J=1.6 \mathrm{~Hz}, 1 \mathrm{H}), 4.67$ (tdt, $J=7.3,5.2,2.4 \mathrm{~Hz}, 1 \mathrm{H}), 4.51-4.47$ (m, $2 \mathrm{H}), 4.34-4.28(\mathrm{~m}, 0.4 \mathrm{H}), 3.98-3.93(\mathrm{~m}, 0.6 \mathrm{H}), 3.66$ (dddd, $J=11.2$, 7.3, 5.5, 2.1 Hz, 0.4H), 3.62-3.57 (m, 0.6 H), 3.54-3.43 (m, $2 \mathrm{H}), 2.46(\mathrm{~d}, J=2.1 \mathrm{~Hz}, 1 \mathrm{H}), 2.45(\mathrm{~d}, J=2.1 \mathrm{~Hz}$, 1 H), 2.35-2.23 (m, $1 \mathrm{H}), 2.21-2.09(\mathrm{~m}, 1.6 \mathrm{H}), 2.05$ (ddd, $J=14.4,5.9,2.7 \mathrm{~Hz}, 0.4 \mathrm{H}), 1.83-1.61$ (m, $3 \mathrm{H})$, $1.59-1.50(\mathrm{~m}, 2 \mathrm{H}), 1.15-1.08(\mathrm{~m}, 4 \mathrm{H}) \mathrm{ppm} ;{ }^{13} \mathrm{C} \mathrm{NMR}\left(151 \mathrm{MHz}, \mathrm{CDCl}_{3}\right) \delta=150.5,150.3,138.7,138.6$, 
$128.5,127.8,127.7,127.6,127.5,105.3,105.1,84.8,84.6,78.5,77.8,77.7,76.7,73.0,72.9,72.6,70.2$, 70.1, 62.0, 60.9, 42.5, 39.2, 37.3, 35.6, 35.5, 32.7, 32.6, 26.1 , 26.0, 18.1, 18.0 ppm; HRMS (ESI-TOF) calcd for $\mathrm{C}_{21} \mathrm{H}_{28} \mathrm{O}_{3} \mathrm{Na}^{+}[\mathrm{M}+\mathrm{Na}]^{+} 351.1931$; Found 351.1935.

$(3 \mathrm{a} R, 5 S, 6 S, 7 \mathrm{a} R)-6-\{[(2 R)-1-\{(2 R, 4 R, 6 S)-6-[3-(B e n z y l o x y) p r o p y l]-4-m e t h y l-3-m e t h y l i d e n e t e t r a-$ hydro-2H-pyran-2-yl\}but-3-yn-2-yl]oxy\}-5-(iodomethyl)hexahydro-2H-furo[3,2-b]pyran-2-one (24a) and $(3 \mathrm{a} R, 5 S, 6 S, 7 \mathrm{a} R)-6-\{[(2 S)-1-\{(2 R, 4 R, 6 S)-6-[3-(b e n z y l o x y) p r o p y l]-4-m e t h y l-3-m e t h y l i d e n e t e t r a-$ hydro-2H-pyran-2-yl\}but-3-yn-2-yl]oxy\}-5-(iodomethyl)hexahydro-2H-furo[3,2-b]pyran-2-one (24b):
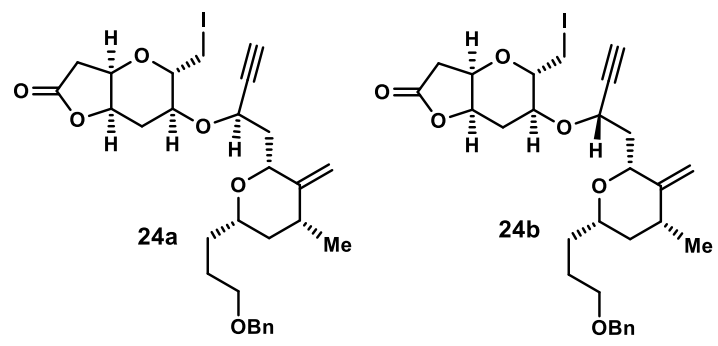

To a stirred solution of alkyne $22(200 \mathrm{mg}, 0.609 \mathrm{mmol}$, 1.0 equiv) in $\mathrm{CH}_{2} \mathrm{Cl}_{2}(5 \mathrm{~mL})$ at $23^{\circ} \mathrm{C}$ was added $\mathrm{Co}_{2}(\mathrm{CO})_{8}$ ( $250 \mathrm{mg}, 0.731 \mathrm{mmol}, 1.2$ equiv) in one portion. After 20 min, a solution of iodide $\mathbf{2 3}^{5}(363 \mathrm{mg}, 1.22 \mathrm{mmol}$, 2.0 equiv) in $\mathrm{CH}_{2} \mathrm{Cl}_{2}(8 \mathrm{~mL})$ was added. The reaction mixture was cooled to $0{ }^{\circ} \mathrm{C}$, and $\mathrm{BF}_{3} \cdot \mathrm{Et}_{2} \mathrm{O}(151 \mu \mathrm{L}, 1.22 \mathrm{mmol}, 2.0$ equiv) was added dropwise. After $0.5 \mathrm{~h}$, the reaction mixture was carefully quenched by the addition of saturated aqueous $\mathrm{NaHCO}_{3}$ solution $(20 \mathrm{~mL})$, and allowed to warm to $23^{\circ} \mathrm{C}$. The aqueous layer was extracted with $\mathrm{CH}_{2} \mathrm{Cl}_{2}(3 \times 20 \mathrm{~mL})$ and the combined organic layers were dried over anhydrous $\mathrm{Na}_{2} \mathrm{SO}_{4}$, filtered, and concentrated under reduced pressure.

To a stirred solution of the so-obtained crude residue in acetone $(20 \mathrm{~mL})$ was added portion wise diammonium cerium(IV) nitrate (CAN) $\left(1.67 \mathrm{~g}, 3.04 \mathrm{mmol}, 5.0\right.$ equiv) at $0{ }^{\circ} \mathrm{C}$. The resulting mixture was allowed to warm to $23^{\circ} \mathrm{C}$ and stirred for $1 \mathrm{~h}$ before it was quenched by the addition of water $(60 \mathrm{~mL})$. The aqueous layer was extracted with EtOAc $(3 \times 30 \mathrm{~mL})$ and the combined organic layers were dried over anhydrous $\mathrm{Na}_{2} \mathrm{SO}_{4}$, filtered, and concentrated under reduced pressure. The obtained residue was purified by flash column chromatography $\left(\mathrm{SiO}_{2}, 10 \rightarrow 40 \%\right.$ ethyl acetate in hexanes) to afford pure cis-isomer $24 \mathbf{a}$ (185 mg, $0.304 \mathrm{mmol}, 50 \%$ yield overall) and trans-isomer $\mathbf{2 b}(59.0 \mathrm{mg}, 0.097 \mathrm{mmol}, 16 \%$ yield overall) as colorless oils (3:1 dr). 24a (cis-isomer): $\mathrm{R}_{\mathrm{f}}=0.50\left(\mathrm{SiO}_{2}, 40 \%\right.$ EtOAc in hexanes); $[\alpha]_{\mathrm{D}}^{23}=+108.3(c=0.6$, $\mathrm{CH}_{2} \mathrm{Cl}_{2}$ ); FT-IR (film) $v_{\max }=3283,3030,2929,2854,1781,1454,1365,1095,1050,904,698 \mathrm{~cm}^{-1} ;{ }^{1} \mathrm{H}$ NMR $\left(600 \mathrm{MHz}, \mathrm{CDCl}_{3}\right) \delta=7.36-7.31(\mathrm{~m}, 4 \mathrm{H}), 7.30-7.26(\mathrm{~m}, 1 \mathrm{H}), 4.80(\mathrm{~d}, J=1.5 \mathrm{~Hz}, 1 \mathrm{H}), 4.77(\mathrm{~d}$, 
$J=1.5 \mathrm{~Hz}, 1 \mathrm{H}), 4.56-4.45(\mathrm{~m}, 5 \mathrm{H}), 3.97(\mathrm{dt}, J=11.0,1.6 \mathrm{~Hz}, 1 \mathrm{H}), 3.83(\mathrm{dt}, J=6.4,4.8 \mathrm{~Hz}, 1 \mathrm{H}), 3.74$ (dddd, $J=11.2,7.4,5.3,2.1 \mathrm{~Hz}, 1 \mathrm{H}), 3.59(\mathrm{ddd}, J=7.7,6.3,3.9 \mathrm{~Hz}, 1 \mathrm{H}), 3.53-3.43(\mathrm{~m}, 3 \mathrm{H}), 3.28(\mathrm{dd}, J=10.8$, $7.8 \mathrm{~Hz}, 1 \mathrm{H}), 2.76(\mathrm{dd}, J=18.5,6.3 \mathrm{~Hz}, 1 \mathrm{H}), 2.67(\mathrm{dd}, J=18.4,1.6 \mathrm{~Hz}, 1 \mathrm{H}), 2.42(\mathrm{~d}, J=2.0 \mathrm{~Hz}, 1 \mathrm{H}), 2.39-$ $2.30(\mathrm{~m}, 1 \mathrm{H}), 2.21-2.10(\mathrm{~m}, 2 \mathrm{H}), 2.06(\mathrm{dt}, J=14.9,4.9 \mathrm{~Hz}, 1 \mathrm{H}), 1.96(\mathrm{ddd}, J=14.2,11.1,2.2 \mathrm{~Hz}, 1 \mathrm{H})$ 1.86-1.74 (m, 2 H), 1.73-1.65 (m, $1 \mathrm{H}), 1.59-1.49$ (m, 2 H), 1.08-1.01 (m, 4H)ppm; ${ }^{13} \mathrm{C}$ NMR (151 MHz, $\left.\mathrm{CDCl}_{3}\right) \delta=175.0,151.5,138.8,128.5,127.8,127.7,104.2,83.4,76.7,76.0,74.5,73.5,73.1,72.9,70.6$, $69.4,63.3,53.6,43.3,38.3,35.7,35.6,32.8,26.6,26.4,18.1,5.5 \mathrm{ppm}$; HRMS (ESI-TOF) calcd for $\mathrm{C}_{29} \mathrm{H}_{37} \mathrm{O}_{6} \mathrm{INa}^{+}[\mathrm{M}+\mathrm{Na}]^{+}$631.1527; Found 631.1528. 24b (trans-isomer): $\mathrm{R}_{\mathrm{f}}=0.4\left(\mathrm{SiO}_{2}\right.$, hexanes/EtOAc 3:2, $v / v) ;[\alpha]_{\mathrm{D}}^{23}=+22.6\left(c=0.50, \mathrm{CH}_{2} \mathrm{Cl}_{2}\right) ;$ FT-IR (film) $v_{\max }=3285,2929,2852,1781,1454,1364,1194,1092$, 1049, 904, $698 \mathrm{~cm}^{-1} ;{ }^{1} \mathrm{H}$ NMR $\left(600 \mathrm{MHz}, \mathrm{CDCl}_{3}\right) \delta=7.36-7.31(\mathrm{~m}, 4 \mathrm{H}), 7.30-7.27(\mathrm{~m}, 1 \mathrm{H}), 4.85$ (brs, $1 \mathrm{H}), 4.81(\mathrm{~d}, J=1.9 \mathrm{~Hz}, 1 \mathrm{H}), 4.63-4.55(\mathrm{~m}, 2 \mathrm{H}), 4.51$ (AB quart, $J=11.5 \mathrm{~Hz}, 2 \mathrm{H}), 4.46(\mathrm{ddd}, J=10.3,4.9$, $2.1 \mathrm{~Hz}, 1 \mathrm{H}), 3.90(\mathrm{dd}, J=10.6,3.0 \mathrm{~Hz}, 1 \mathrm{H}), 3.73-3.64(\mathrm{~m}, 1 \mathrm{H}), 3.56$ (td, $J=6.7,4.0 \mathrm{~Hz}, 1 \mathrm{H}), 3.49$ (dddd, $J=15.8,13.2,9.0,6.5 \mathrm{~Hz}, 3 \mathrm{H}), 3.36(\mathrm{dd}, J=10.9,4.0 \mathrm{~Hz}, 1 \mathrm{H}), 3.30(\mathrm{dd}, J=10.9,6.7 \mathrm{~Hz}, 1 \mathrm{H}), 2.79-2.63$ (m, $2 \mathrm{H}), 2.56$ (ddd, $J=14.4,6.1,4.7 \mathrm{~Hz}, 1 \mathrm{H}), 2.48$ (d, $J=2.0 \mathrm{~Hz}, 1 \mathrm{H}), 2.35-2.23(\mathrm{~m}, 1 \mathrm{H}), 2.19-2.09$ (m, 2H), 2.04 (ddd, $J=13.2,10.3,3.1 \mathrm{~Hz}, 1 \mathrm{H}), 1.79$ (dddd, $J=17.2,9.2,6.8,4.3 \mathrm{~Hz}, 2 \mathrm{H}), 1.71-1.61(\mathrm{~m}, 1 \mathrm{H})$, 1.55-1.48 (m, 2 H), 1.11-1.05 (m, 4H) ppm; ${ }^{13} \mathrm{C}$ NMR (151 MHz, $\left.\mathrm{CDCl}_{3}\right) \delta=174.7,150.7,138.7,128.5$, $127.8,127.7,104.8,82.8,77.5,76.2,75.2,74.9,74.0,73.1,73.0,70.4,69.2,67.9,43.2,37.9,35.8,34.7$, 32.5, 30.3, 26.2, 18.1, 6.0ppm; HRMS (ESI-TOF) calcd for $\mathrm{C}_{29} \mathrm{H}_{37} \mathrm{O}_{6} \mathrm{INa}^{+}[\mathrm{M}+\mathrm{Na}]^{+} 631.1527$; Found 631.1528.

Methyl 3,7-anhydro-6-O-[(2R)-1-\{(2R,4R,6S)-6-[3-(benzyloxy)propyl]-4-methyl-3-methylidenetetrahydro-2H-pyran-2-yl\}but-3-yn-2-yl]-2,5,8-trideoxy-8-iodo-a-D-altro-octofuranoside (25): To a stirred

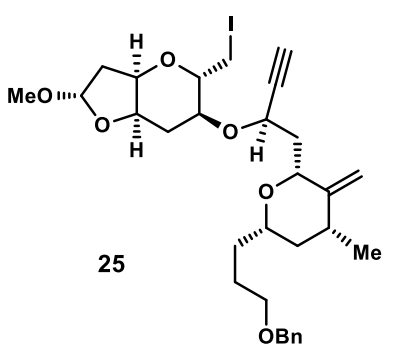

solution of lactone $\mathbf{2 4 b}\left(200 \mathrm{mg}, 0.329 \mathrm{mmol}, 1.0\right.$ equiv) in $\mathrm{CH}_{2} \mathrm{Cl}_{2}(10 \mathrm{~mL})$ at $-78^{\circ} \mathrm{C}$ was added dropwise DIBAL-H $(1.0 \mathrm{M}$ in toluene, $395 \mu \mathrm{L}, 0.395 \mathrm{mmol}$, 1.2 equiv). The resulting mixture was warmed to $-20^{\circ} \mathrm{C}$ and stirred for $1 \mathrm{~h}$ before it was diluted with ethyl acetate $(20 \mathrm{~mL})$ and quenched by the addition of saturated aqueous solution of Rochelle salt solution $(40 \mathrm{~mL})$, allowed to 
warm to $23^{\circ} \mathrm{C}$, and stirred for $2 \mathrm{~h}$ until the reaction mixture became a clear solution. The aqueous layer was extracted with EtOAc $(3 \times 10 \mathrm{~mL})$, and the combined organic layers were dried over anhydrous $\mathrm{Na}_{2} \mathrm{SO}_{4}$, filtered, and concentrated under reduced pressure.

The so-obtained crude residue was dissolved in $\mathrm{MeOH}(8 \mathrm{~mL})$ and $p$ - $\mathrm{TsOH} \cdot \mathrm{H}_{2} \mathrm{O}(11.2 \mathrm{mg}$, $0.0658 \mathrm{mmol}, 0.2$ equiv) was added at $23^{\circ} \mathrm{C}$. After $0.5 \mathrm{~h}$, the reaction mixture was quenched by the addition of saturated aqueous $\mathrm{NaHCO}_{3}(6 \mathrm{~mL})$ and diluted with water $(6 \mathrm{~mL})$. The aqueous layer was extracted with EtOAc $(3 \times 10 \mathrm{~mL})$, and the combined organic layers were dried over anhydrous $\mathrm{Na}_{2} \mathrm{SO}_{4}$, filtered, and concentrated under reduced pressure. The obtained residue was purified by flash column chromatography $\left(\mathrm{SiO}_{2}, 5 \rightarrow 20 \%\right.$ EtOAc in hexanes) to afford pure acetal $25(154 \mathrm{mg}, 0.247 \mathrm{mmol}, 75 \%$ yield overall) as a colorless oil. 25: $\mathrm{R}_{\mathrm{f}}=0.60\left(\mathrm{SiO}_{2}\right.$, hexanes/EtOAc 2:1, v/v); $[\alpha]_{\mathrm{D}}^{23}=+86.2\left(c=1.5, \mathrm{CH}_{2} \mathrm{Cl}_{2}\right)$; FT-IR (film) $v_{\max }=3286,2927,2853,1454,1366,1211,1114,1093,1057,904,698 \mathrm{~cm}^{-1} ;{ }^{1} \mathrm{H} \mathrm{NMR}\left(600 \mathrm{MHz}, \mathrm{CDCl}_{3}\right)$ $\delta=7.36-7.31(\mathrm{~m}, 4 \mathrm{H}), 7.30-7.27(\mathrm{~m}, 1 \mathrm{H}), 5.13(\mathrm{dd}, J=4.3,3.1 \mathrm{~Hz}, 1 \mathrm{H}), 4.83(\mathrm{~d}, J=1.8 \mathrm{~Hz}, 1 \mathrm{H}), 4.79(\mathrm{~d}$, $J=1.8 \mathrm{~Hz}, 1 \mathrm{H}), 4.50(\mathrm{~s}, 2 \mathrm{H}), 4.48(\mathrm{q}, J=5.2 \mathrm{~Hz}, 1 \mathrm{H}), 4.44(\mathrm{dt}, J=11.0,2.2 \mathrm{~Hz}, 1 \mathrm{H}), 4.13(\mathrm{q}, J=4.5 \mathrm{~Hz}$, 1 H), 4.02-3.96 (m, $1 \mathrm{H}), 3.71-3.60(\mathrm{~m}, 2 \mathrm{H}), 3.55-3.42(\mathrm{~m}, 4 \mathrm{H}), 3.29(\mathrm{~s}, 3 \mathrm{H}), 3.22(\mathrm{dd}, J=10.5,8.1 \mathrm{~Hz}$, $1 \mathrm{H}), 2.39(\mathrm{~d}, J=2.0 \mathrm{~Hz}, 1 \mathrm{H}), 2.30-2.21(\mathrm{~m}, 3 \mathrm{H}), 2.14(\mathrm{ddd}, J=14.1,11.1,2.1 \mathrm{~Hz}, 1 \mathrm{H}), 2.06-1.90$ (m, $3 \mathrm{H})$, $1.87-1.79(\mathrm{~m}, 1 \mathrm{H}), 1.77$ (ddd, $J=12.8,4.6,2.1 \mathrm{~Hz}, 1 \mathrm{H}), 1.68$ (ddq, $J=12.8,9.5,6.7 \mathrm{~Hz}, 1 \mathrm{H}), 1.55$ (ddd, $J=8.8,7.3,4.3 \mathrm{~Hz}, 2 \mathrm{H}), 1.11-1.05(\mathrm{~m}, 4 \mathrm{H}) \mathrm{ppm} ;{ }^{13} \mathrm{C} \mathrm{NMR}\left(151 \mathrm{MHz}, \mathrm{CDCl}_{3}\right) \delta=151.1,138.6,128.5$, 127.7, 127.6, 104.5, 104.1, 83.8, 74.4, 73.2, 73.1, 73.0, 72.9, 72.6, 63.1, 54.9, 43.3, 40.0, 38.3, 35.9, 33.0, 27.3, 26.4, 18.1, 7.9 ppm; HRMS (ESI-TOF) calcd for $\mathrm{C}_{30} \mathrm{H}_{41} \mathrm{O}_{6} \mathrm{INa}^{+}[\mathrm{M}+\mathrm{Na}]^{+}$647.1840; Found 647.1842.

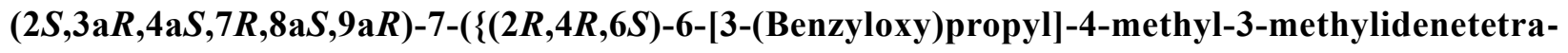
hydro-2H-pyran-2-yl\}methyl)-2-methoxy-6-methylidenedecahydrofuro[3,2-b]pyrano[2,3-e]pyran (27):

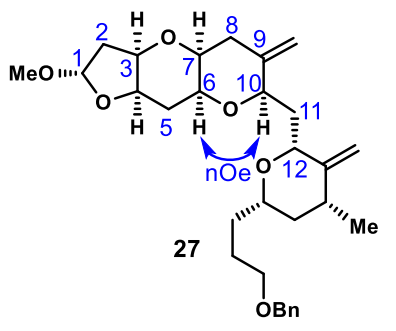

To a stirred solution of 25 (108 $\mathrm{mg}, 0.173 \mathrm{mmol}, 1.0$ equiv) in THF ( $5 \mathrm{~mL})$ at $0^{\circ} \mathrm{C}$ was added $\mathrm{KO} t \mathrm{Bu}(38.8 \mathrm{mg}, 0.346 \mathrm{mmol}, 2.0$ equiv). The resulting mixture was allowed to warm to $23^{\circ} \mathrm{C}$ and stirred for $10 \mathrm{~min}$ before it was diluted with hexanes $(30 \mathrm{~mL})$ and EtOAc $(15 \mathrm{~mL})$ and filtered through a pad of $\mathrm{SiO}_{2}$. The filtrate was concentrated under reduced pressure to give crude 
exocyclic olefin 26 (90.2 $\mathrm{mg}, 0.173 \mathrm{mmol}$, quantitative yield), which was used in the next step without further purification.

To a stirred solution of the obtained exocyclic olefin $26(90.2 \mathrm{mg}, 0.173 \mathrm{mmol}, 1.0$ equiv) in toluene $(10 \mathrm{~mL})$ at $23{ }^{\circ} \mathrm{C}$ were added $n-\mathrm{Bu}_{3} \mathrm{SnH}(100 \mu \mathrm{L}, 0.346 \mathrm{mmol}, 2.0$ equiv) and AIBN (14.2 $\mathrm{mg}, 0.0865 \mathrm{mmol}$, 0.5 equiv). The resulting mixture was transferred and submerged into a preheated oil bath $\left(90^{\circ} \mathrm{C}\right)$ and stirred for $2 \mathrm{~h}$ before it was allowed to cool to $23{ }^{\circ} \mathrm{C}$. The resulting mixture was concentrated under reduced pressure. Flash column chromatography $\left(\mathrm{SiO}_{2}\right.$, hexanes/EtOAc 20:1, v/v $\left.\rightarrow 2: 1, v / v\right)$ of the residue afforded the corresponding organotin intermediate $(80.1 \mathrm{mg}, 0.102 \mathrm{mmol}, 58 \%$ yield) as a colorless oil.

To a stirred solution of the residue obtained above (mixture of isomers, $80.1 \mathrm{mg}, 0.102 \mathrm{mmol}$, 1.0 equiv) in $\mathrm{MeOH}(4 \mathrm{~mL})$ at $23^{\circ} \mathrm{C}$ was added $p$ - $\mathrm{TsOH} \cdot \mathrm{H}_{2} \mathrm{O}(17.5 \mathrm{mg}, 0.102 \mathrm{mmol}, 1.0$ equiv). The resulting mixture was stirred for $45 \mathrm{~min}$ and then diluted with saturated aqueous $\mathrm{NaHCO}_{3}$ solution $(10 \mathrm{~mL})$. The aqueous layer was extracted with EtOAc $(3 \times 10 \mathrm{~mL})$. The combined organic layers were washed with brine $(10 \mathrm{~mL})$, dried over $\mathrm{Na}_{2} \mathrm{SO}_{4}$, filtered, and concentrated under reduced pressure. Flash column chromatography $\left(\mathrm{SiO}_{2}, 5 \rightarrow 20 \%\right.$ EtOAc in hexanes) of the residue afforded $27(40.7 \mathrm{mg}, 0.0816 \mathrm{mmol}, 80 \%$ yield) as a colorless oil. NOE studies indicate the cis-relation between $\mathrm{H} 6$ and H10. 27: $\mathrm{R}_{\mathrm{f}}=0.20\left(\mathrm{SiO}_{2}\right.$, hexanes/EtOAc 5:1, v/v); $[\alpha]_{\mathrm{D}}^{23}=+67.8\left(c=2.0, \mathrm{CH}_{2} \mathrm{Cl}_{2}\right)$; FT-IR (film) $v_{\max }=2953,2850,1651,1454,1364$, 1194, 1094, 1028, 897, $697 \mathrm{~cm}^{-1} ;{ }^{1} \mathrm{H}$ NMR $\left(600 \mathrm{MHz}, \mathrm{C}_{6} \mathrm{D}_{6}\right) \delta=7.33(\mathrm{dd}, J=7.4,1.5 \mathrm{~Hz}, 2 \mathrm{H}), 7.19(\mathrm{td}$, $J=7.6,1.8 \mathrm{~Hz}, 2 \mathrm{H}), 7.11(\mathrm{td}, J=7.2,1.4 \mathrm{~Hz}, 1 \mathrm{H}), 5.02(\mathrm{dd}, J=5.7,2.8 \mathrm{~Hz}, 1 \mathrm{H}), 4.87$ (brs, $1 \mathrm{H}), 4.84$ (brs, 1 H), 4.82 (brs, $1 \mathrm{H}), 4.73(\mathrm{~d}, J=1.9 \mathrm{~Hz}, 1 \mathrm{H}), 4.37(\mathrm{~s}, 2 \mathrm{H}), 4.34(\mathrm{~d}, J=10.5 \mathrm{~Hz}, 1 \mathrm{H}), 4.28-4.23(\mathrm{~m}, 1 \mathrm{H})$, $3.73(\mathrm{dt}, J=5.4,3.3 \mathrm{~Hz}, 1 \mathrm{H}), 3.66(\mathrm{ddd}, J=5.7,3.2,1.8 \mathrm{~Hz}, 1 \mathrm{H}), 3.40$ (dddd, $J=15.4,9.0,6.4,2.8 \mathrm{~Hz}, 3 \mathrm{H})$, $3.31(\mathrm{ddd}, J=5.5,3.6,2.1 \mathrm{~Hz}, 1 \mathrm{H}), 3.15(\mathrm{~s}, 3 \mathrm{H}), 2.95(\mathrm{td}, J=4.4,2.0 \mathrm{~Hz}, 1 \mathrm{H}), 2.54$ (dd, $J=14.0,4.1 \mathrm{~Hz}$, $1 \mathrm{H}), 2.36(\mathrm{dt}, J=15.0,3.6 \mathrm{~Hz}, 1 \mathrm{H}), 2.31-2.23(\mathrm{~m}, 3 \mathrm{H}), 2.13(\mathrm{ddd}, J=13.5,10.7,2.4 \mathrm{~Hz}, 1 \mathrm{H}), 2.06(\mathrm{ddd}$, $J=14.1,6.1,2.8 \mathrm{~Hz}, 1 \mathrm{H}), 1.99$ (ddt, $J=12.6,6.3,3.3 \mathrm{~Hz}, 1 \mathrm{H}), 1.90$ (dddd, $J=15.9,8.5,4.6,1.7 \mathrm{~Hz}, 1 \mathrm{H})$, $1.77(\mathrm{dtd}, J=13.3,6.6,3.0 \mathrm{~Hz}, 1 \mathrm{H}), 1.69(\mathrm{dt}, J=14.9,5.4 \mathrm{~Hz}, 1 \mathrm{H}), 1.61(\mathrm{dtt}, J=13.9,9.2,4.0 \mathrm{~Hz}, 1 \mathrm{H}), 1.49$ (dddd, $J=13.7,10.1,6.3,4.2 \mathrm{~Hz}, 1 \mathrm{H}), 1.40(\mathrm{ddd}, J=12.5,4.6,2.1 \mathrm{~Hz}, 1 \mathrm{H}), 1.05-0.99(\mathrm{~m}, 1 \mathrm{H}), 0.96(\mathrm{~d}$, $J=6.5 \mathrm{~Hz}, 3 \mathrm{H}) \mathrm{ppm} ;{ }^{13} \mathrm{C} \mathrm{NMR}\left(151 \mathrm{MHz}, \mathrm{C}_{6} \mathrm{D}_{6}\right) \delta=152.6,144.8,139.5,128.6,128.4,127.7,108.9,104.7$, 
104.0, 77.1, 77.0, 74.9, 74.4, 73.4, 73.0, 72.3, 70.8, 70.6, 55.1, 43.4, 41.4, 38.3, 36.0, 35.2, 33.0, 30.6, 26.7, 18.2 ppm; HRMS (ESI-TOF) calcd for $\mathrm{C}_{30} \mathrm{H}_{42} \mathrm{O}_{6} \mathrm{Na}^{+}[\mathrm{M}+\mathrm{Na}]^{+}$521.2874; Found 521.2874.

\section{Methyl (16S)-3,7:6,10:12,16-trianhydro-16-[3-(benzyloxy)propyl]-2,5,8,11,14,15-hexadeoxy-14-} methyl-D-xylo-ק-L-galactadecofuranoside-9,13-diulose (28): Through a stirred solution of olefin 27

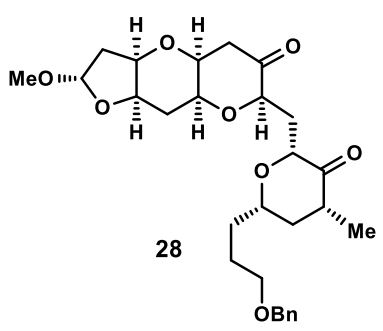
(30.0 mg, $0.0602 \mathrm{mmol}, 1.0$ equiv) in $\mathrm{CH}_{2} \mathrm{Cl}_{2}(6 \mathrm{~mL})$ at $-78^{\circ} \mathrm{C}$ was bubbled freshly generated ozone. After the color of the reaction mixture changed to light blue (ca. $5 \mathrm{~min}$ ), the resulting mixture was quenched by the addition of dimethyl sulfide $\left(44.0 \mu \mathrm{L}, 0.602 \mathrm{mmol}, 10.0\right.$ equiv), and allowed to warm to $23^{\circ} \mathrm{C}$. After stirring for $10 \mathrm{~h}$, the solvent was removed under reduced pressure, and the obtained residue was purified by flash column chromatography $\left(\mathrm{SiO}_{2}, 20 \rightarrow 40 \%\right.$ ethyl acetate in hexanes) to afford pure diketone $28(28.4 \mathrm{mg}, 0.0566 \mu \mathrm{mol}, 94 \%$ yield $)$ as a colorless oil. 3: $\mathrm{R}_{\mathrm{f}}=0.40\left(\mathrm{SiO}_{2}\right.$, hexanes/EtOAc 2:1, v/v); $[\alpha]_{\mathrm{D}}^{23}=+74.2\left(c=0.60, \mathrm{CH}_{2} \mathrm{Cl}_{2}\right)$; FT-IR (film) $v_{\max }=2928,2854,1724,1454,1362$, 1262, 1094, 1046, $740 \mathrm{~cm}^{-1}$; ${ }^{1} \mathrm{H}$ NMR $\left(600 \mathrm{MHz}, \mathrm{C}_{6} \mathrm{D}_{6}\right) \delta$ 7.17-6.98 (m, 4 H), 6.95-6.79 (m, $\left.1 \mathrm{H}\right), 4.75(\mathrm{dd}$, $J=5.6,2.9 \mathrm{~Hz}, 1 \mathrm{H}), 4.18(\mathrm{~s}, 2 \mathrm{H}), 3.85(\mathrm{ddd}, J=9.9,3.8,1.0 \mathrm{~Hz}, 1 \mathrm{H}), 3.77(\mathrm{dd}, J=9.5,3.9 \mathrm{~Hz}, 1 \mathrm{H}), 3.48$ (dt, $J=5.3,3.8 \mathrm{~Hz}, 1 \mathrm{H}), 3.32$ (dddt, $J=11.2,8.9,5.0,2.9 \mathrm{~Hz}, 1 \mathrm{H}), 3.21-3.13(\mathrm{~m}, 3 \mathrm{H}), 2.96(\mathrm{dt}, J=5.0$, $2.5 \mathrm{~Hz}, 1 \mathrm{H}), 2.94(\mathrm{~s}, 3 \mathrm{H}), 2.76(\mathrm{td}, J=4.9,2.3 \mathrm{~Hz}, 1 \mathrm{H}), 2.39(\mathrm{dd}, J=15.5,4.4 \mathrm{~Hz}, 1 \mathrm{H}), 2.35-2.28(\mathrm{~m}, 2 \mathrm{H})$, $2.04(\mathrm{dt}, J=14.6,4.3 \mathrm{~Hz}, 1 \mathrm{H}), 1.96-1.89(\mathrm{~m}, 2 \mathrm{H}), 1.81(\mathrm{ddd}, J=14.2,6.2,2.9 \mathrm{~Hz}, 1 \mathrm{H}), 1.64-1.53(\mathrm{~m}, 2 \mathrm{H})$, $1.47-1.38(\mathrm{~m}, 2 \mathrm{H}), 1.37-1.31(\mathrm{~m}, 1 \mathrm{H}), 1.26(\mathrm{ddd}, J=13.1,6.2,1.9 \mathrm{~Hz}, 1 \mathrm{H}), 1.24-1.21(\mathrm{~m}, 1 \mathrm{H}), 0.98$ (td, $J=12.9,11.0 \mathrm{~Hz}, 1 \mathrm{H}), 0.74(\mathrm{~d}, J=6.6 \mathrm{~Hz}, 3 \mathrm{H}) \mathrm{ppm} ;{ }^{13} \mathrm{C} \mathrm{NMR}\left(151 \mathrm{MHz}, \mathrm{C}_{6} \mathrm{D}_{6}\right) \delta 207.4,205.8,139.4,129.9$, $128.7,127.8,104.7,79.0,77.7,77.1,76.6,73.7,73.1,72.2,70.32,70.26,55.2,43.4,42.39,42.37,41.1$, 32.3, 30.4, 30.1, 26.6, $14.6 \mathrm{ppm}$; HRMS (ESI-TOF) calcd for $\mathrm{C}_{28} \mathrm{H}_{38} \mathrm{O}_{8} \mathrm{Na}^{+}[\mathrm{M}+\mathrm{Na}]^{+}$525.2459; Found 525.2462.

Methyl 3,7-anhydro-2,5,8-trideoxy-8-iodo- $\alpha$-D-altro-octofuranoside (S2): To a stirred solution of 23 

(10.2 g, 34.2 mmol, 1.0 equiv) in $\mathrm{CH}_{2} \mathrm{Cl}_{2}(300 \mathrm{~mL})$ at $-78^{\circ} \mathrm{C}$ was added dropwise DIBAL-H (1.0 M in toluene, $85.5 \mathrm{~mL}, 85.5 \mathrm{mmol}, 2.5$ equiv). The resulting mixture was 
allowed to slowly warm to $-20^{\circ} \mathrm{C}$ and stirred for $1 \mathrm{~h}$. The mixture was acidified by addition of $\mathrm{HCl}(3 \mathrm{M}$ in $\mathrm{MeOH}, 150 \mathrm{~mL}, 450 \mathrm{mmol}, 13.2$ equiv), the resulting mixture was allowed to warm to $23^{\circ} \mathrm{C}$ and stirred for $8 \mathrm{~h}$ before it was diluted with $\mathrm{H}_{2} \mathrm{O}(200 \mathrm{~mL})$. The layers were separated, and the aqueous layer was extracted with $\mathrm{CH}_{2} \mathrm{Cl}_{2}(3 \times 300 \mathrm{~mL})$. The combined organic layers were washed with $\mathrm{NaHCO}_{3}$ solution $(100 \mathrm{~mL}$, sat. aq.), dried over $\mathrm{Na}_{2} \mathrm{SO}_{4}$, filtered, and concentrated under reduced pressure. Flash column chromatography $\left(\mathrm{SiO}_{2}\right.$, hexanes/EtOAc 1:1, v/v $\left.\rightarrow 1: 2, v / v\right)$ of the residue afforded $\mathbf{S 2}(7.63 \mathrm{~g}, 24.3 \mathrm{mmol}, 71 \%$ yield $)$ and C1-epi-S2 (1.51 g, $4.79 \mathrm{mmol}, 14 \%$ yield) as colorless oils, respectively. The configurations of the newly generated stereocenters were confirmed by nOe studies (see spectrum part). S2: $\mathrm{R}_{\mathrm{f}}=0.40\left(\mathrm{SiO}_{2}\right.$, hexanes/EtOAc 1:1, v/v); $[\alpha]_{\mathrm{D}}^{23}=+97.1\left(c=1.0, \mathrm{CHCl}_{3}\right)$; FTIR (film): $v_{\max } 3504,2926,2831,1430,1368$, $1345,1248,1212,1184,1140,1096,1055,1038,1018,993,969,950,916,890,846,800 \mathrm{~cm}^{-1} ;{ }^{1} \mathrm{H}$ NMR $\left(600 \mathrm{MHz}, \mathrm{C}_{6} \mathrm{D}_{6}\right): \delta 4.74(\mathrm{dd}, J=6.0,3.5 \mathrm{~Hz}, 1 \mathrm{H}), 3.82$ (dddd, $\left.J=9.4,5.4,2.8,1.1 \mathrm{~Hz}, 1 \mathrm{H}\right), 3.59-3.48(\mathrm{~m}$, $3 \mathrm{H}), 3.44-3.32(\mathrm{~m}, 1 \mathrm{H}), 3.09$ (s, $3 \mathrm{H}), 2.66$ (dd, $J=10.7,9.6 \mathrm{~Hz}, 1 \mathrm{H}), 2.54$ (dd, $J=10.7,5.3 \mathrm{~Hz}, 1 \mathrm{H}), 2.15$ (ddd, $J=14.6,6.0,0.8 \mathrm{~Hz}, 1 \mathrm{H}), 1.91$ (ddt, $J=15.3,2.5,1.1 \mathrm{~Hz}, 1 \mathrm{H}), 1.83(\mathrm{ddd}, J=14.6,5.2,3.5 \mathrm{~Hz}, 1 \mathrm{H})$, $1.20(\mathrm{dt}, J=15.6,3.7 \mathrm{~Hz}, 1 \mathrm{H}) . \mathrm{ppm} ;{ }^{13} \mathrm{C} \mathrm{NMR}\left(151 \mathrm{MHz}, \mathrm{C}_{6} \mathrm{D}_{6}\right): \delta 104.5,78.0,73.7,70.4,65.3,55.5,41.4$, 26.9, 3.6ppm; HRMS (ESI-TOF) m/z: [M+Na] ${ }^{+}$Calcd. for $\mathrm{C}_{9} \mathrm{H}_{15} \mathrm{IO}_{4} \mathrm{Na}^{+}$336.9907; Found 336.9903.

Methyl 3,7-anhydro-2,5,8-trideoxy-8-iodo-6-O-(triethylsilyl)- $\alpha$-D-altro-octofuranoside (34): To a

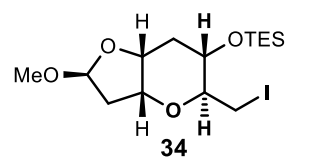
stirred solution of $\mathbf{S 2}\left(7.60 \mathrm{~g}, 24.3 \mathrm{mmol}, 1.0\right.$ equiv) in $\mathrm{CH}_{2} \mathrm{Cl}_{2}(100 \mathrm{~mL})$ at $0{ }^{\circ} \mathrm{C}$ was added imidazole $(3.31 \mathrm{~g}, 48.6 \mathrm{mmol}, 2.0$ equiv) and TESCl $(4.00 \mathrm{~mL}, 31.6 \mathrm{mmol}$, 1.3 equiv). The resulting mixture was allowed to slowly warm to $23{ }^{\circ} \mathrm{C}$ and stirred for $1 \mathrm{~h}$ before it was quenched by the addition of sat. aq. $\mathrm{NaHCO}_{3}$ solution $(50 \mathrm{~mL})$. The layers were separated, and the aqueous layer was extracted with $\mathrm{CH}_{2} \mathrm{Cl}_{2}(3 \times 100 \mathrm{~mL})$. The combined organic layers were washed with brine $(100 \mathrm{~mL})$, dried over $\mathrm{Na}_{2} \mathrm{SO}_{4}$, filtered, and concentrated under reduced pressure. Flash column chromatography $\left(\mathrm{SiO}_{2}\right.$, hexanes/EtOAc 20:1, v/v $\rightarrow$ 2:1, v/v) of the residue afforded $34(10.4 \mathrm{~g}, 24.3 \mathrm{mmol}$, quant. yield) as colorless oil. 34: $\mathrm{R}_{\mathrm{f}}=0.70\left(\mathrm{SiO}_{2}\right.$, hexanes/EtOAc 5:1, v/v); $[\alpha]_{\mathrm{D}}^{23}=+93.5\left(c=1.0, \mathrm{CHCl}_{3}\right)$; FTIR (film): $v_{\max } 2954,2911,2877,1458,1369,1343,1212,1177,1118,1100,1043,1018,1002,918,878$, 805, 744, $728 \mathrm{~cm}^{-1} ;{ }^{1} \mathrm{H}$ NMR $\left(600 \mathrm{MHz}, \mathrm{CDCl}_{3}\right): \delta 5.10(\mathrm{dd}, J=5.6,1.7 \mathrm{~Hz}, 1 \mathrm{H}), 4.52(\mathrm{dt}, J=7.3,4.7 \mathrm{~Hz}$, 
$1 \mathrm{H}), 4.15(\mathrm{q}, J=6.0 \mathrm{~Hz}, 1 \mathrm{H}), 3.63(\mathrm{td}, J=7.8,5.7 \mathrm{~Hz}, 1 \mathrm{H}), 3.42(\mathrm{dd}, J=10.4,3.0 \mathrm{~Hz}, 1 \mathrm{H}), 3.35(\mathrm{ddd}, J=7.9$, 6.7, 3.0 Hz, 1 H), 3.31 (s, $3 \mathrm{H}), 3.24$ (dd, $J=10.4,6.7 \mathrm{~Hz}, 1 \mathrm{H}), 2.29-2.20$ (m, $2 \mathrm{H}), 2.17$ (ddd, $J=14.4,7.3$, $1.7 \mathrm{~Hz}, 1 \mathrm{H}), 1.82(\mathrm{ddd}, J=13.8,7.7,6.2 \mathrm{~Hz}, 1 \mathrm{H}), 0.96(\mathrm{t}, J=8.0 \mathrm{~Hz}, 9 \mathrm{H}), 0.62(\mathrm{qd}, J=7.9,1.4 \mathrm{~Hz}, 6 \mathrm{H}) \mathrm{ppm}$; ${ }^{13} \mathrm{C}$ NMR $\left(151 \mathrm{MHz}, \mathrm{CDCl}_{3}\right): \delta$ 104.1, 75.3, 74.0, 73.6, 68.2, 55.0, 39.1, 34.2, 8.7, 7.0, 5.1 ppm; HRMS (ESI-TOF) $m / z$ : $[\mathrm{M}+\mathrm{Na}]^{+}$Calcd. for $\mathrm{C}_{15} \mathrm{H}_{29} \mathrm{IO}_{4} \mathrm{SiNa}^{+}$451.0772; Found 451.0774.

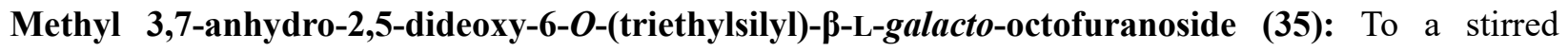

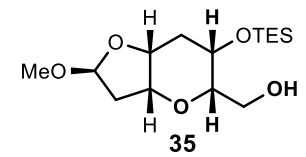
solution of $34\left(9.30 \mathrm{~g}, 21.7 \mathrm{mmol}, 1.0\right.$ equiv) in THF $(60 \mathrm{~mL})$ at $0{ }^{\circ} \mathrm{C}$ was added $\mathrm{KO} t$ - $\mathrm{Bu}(3.65 \mathrm{~g}, 32.6 \mathrm{mmol}, 1.5$ equiv). The resulting mixture was allowed to warm to $23^{\circ} \mathrm{C}$ and stirred for $5 \mathrm{~min}$ before it was diluted with hexanes $(100 \mathrm{~mL})$ and EtOAc $(30 \mathrm{~mL})$, and filtered through a pad of $\mathrm{SiO}_{2}$. The filtrate was concentrated under reduced pressure to give crude exocyclic olefin $26(6.50 \mathrm{~g}, 21.7 \mathrm{mmol}$, quantitative yield), which was used in the next step without further purification.

To a stirred solution of the above obtained olefin intermediate $(6.50 \mathrm{~g}, 21.7 \mathrm{mmol}, 1.0$ equiv) in THF $(100 \mathrm{~mL})$ at $0{ }^{\circ} \mathrm{C}$ were added $\mathrm{BH}_{3} \cdot \mathrm{Me}_{2} \mathrm{~S}(2.0 \mathrm{M}$ in THF; $12.0 \mathrm{~mL}, 23.9 \mathrm{mmol}, 1.1$ equiv), the resulting mixture was allowed to warm to $23^{\circ} \mathrm{C}$ and stirred for $0.5 \mathrm{~h}$ before it was quenched by the addition of $\mathrm{H}_{2} \mathrm{O}(150 \mathrm{~mL})$. To this mixture was added $\mathrm{NaBO}_{3}(9.46 \mathrm{~g}, 95.6 \mathrm{mmol}, 4.4$ equiv) and the resulting mixture was further stirred for $6 \mathrm{~h}$. The reaction mixture was then extracted with EtOAc $(3 \times 100 \mathrm{~mL})$, the combined organic extracts were washed with brine $(100 \mathrm{~mL})$, dried over $\mathrm{Na}_{2} \mathrm{SO}_{4}$, filtered, and concentrated under reduced pressure. Flash column chromatography $\left(\mathrm{SiO}_{2}\right.$, hexanes/EtOAc 10:1, v/v $\left.\rightarrow 1: 2, v / v\right)$ afforded $35(5.32 \mathrm{~g}$, $16.7 \mathrm{mmol}, 77 \%$ yield for the two steps $)$ as a colorless oil. 35: $\mathrm{R}_{\mathrm{f}}=0.40\left(\mathrm{SiO}_{2}\right.$, hexanes/EtOAc $\left.1: 1, v / v\right)$; $[\alpha]_{\mathrm{D}}^{23}=+75.3$ ( $c=1.0$, EtOAc); FT-IR (film): $v_{\max } 3473,2953,2911,2877,1459,1419,1374,1238,1181$, $1143,1097,1022,926,867,783,741,726 \mathrm{~cm}^{-1} ;{ }^{1} \mathrm{H}$ NMR (600 MHz, $\left.\mathrm{C}_{6} \mathrm{D}_{6}\right): \delta 5.15(\mathrm{dd}, J=5.8,3.7 \mathrm{~Hz}, 1 \mathrm{H})$, $3.86(\mathrm{dd}, J=11.1,7.2 \mathrm{~Hz}, 1 \mathrm{H}), 3.72(\mathrm{dt}, J=5.1,2.6 \mathrm{~Hz}, 1 \mathrm{H}), 3.66-3.56(\mathrm{~m}, 2 \mathrm{H}), 3.51-3.40(\mathrm{~m}, 1 \mathrm{H}), 3.25$ (s, $3 \mathrm{H}), 3.03$ (ddd, $J=7.2,4.6,1.8 \mathrm{~Hz}, 1 \mathrm{H}), 2.26$ (ddt, $J=14.3,5.8,0.8 \mathrm{~Hz}, 1 \mathrm{H}), 2.07$ (dt, $J=15.1,2.9 \mathrm{~Hz}$, $1 \mathrm{H}), 2.01$ (ddd, $J=14.3,5.6,3.7 \mathrm{~Hz}, 1 \mathrm{H}), 1.82(\mathrm{~d}, J=6.8 \mathrm{~Hz}, 1 \mathrm{H}), 1.34$ (ddd, $J=15.1,4.8,4.1 \mathrm{~Hz}, 1 \mathrm{H})$, $1.02(\mathrm{t}, J=7.9 \mathrm{~Hz}, 9 \mathrm{H}), 0.59$ (qd, $J=7.9,5.9 \mathrm{~Hz}, 6 \mathrm{H}) \mathrm{ppm} ;{ }^{13} \mathrm{C} \mathrm{NMR}\left(151 \mathrm{MHz}, \mathrm{C}_{6} \mathrm{D}_{6}\right): \delta 105.1,78.7,77.2$, 73.1, 64.4, 63.6, 55.2, 41.7, 32.9, 7.1, 5.4 ppm; HRMS (ESI-TOF) $m / z$ : $[\mathrm{M}+\mathrm{Na}]^{+}$Calcd. for $\mathrm{C}_{15} \mathrm{H}_{30} \mathrm{O}_{5} \mathrm{SiNa}^{+}$ 
341.1755; Found 341.1760.

Methyl (8S)-2,6-anhydro-4,7-dideoxy-3-O-(triethylsilyl)-D-galacto-octodialdo-8,5-furanoside (36): To

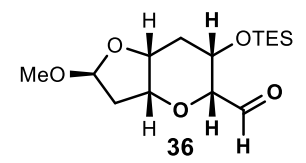
a stirred solution of alcohol 35 (4.70 g, $14.7 \mathrm{mmol}, 1.0$ equiv) in dry $\mathrm{CH}_{2} \mathrm{Cl}_{2}(50 \mathrm{~mL})$ at $0{ }^{\circ} \mathrm{C}$ was added Dess-Martin periodinane $(9.37 \mathrm{~g}, 22.1 \mathrm{mmol}, 1.5$ equiv). The resulting mixture was allowed to warm to $23^{\circ} \mathrm{C}$ and stirred for $1 \mathrm{~h}$ before it was quenched by the addition of sat. aq. $\mathrm{NaHCO}_{3}$ solution $(30 \mathrm{~mL})$ and a sat. aq. $\mathrm{Na}_{2} \mathrm{~S}_{2} \mathrm{O}_{3}$ solution $(50 \mathrm{~mL})$. The layers were separated, and the aqueous layer was extracted with $\mathrm{CH}_{2} \mathrm{Cl}_{2}(3 \times 50 \mathrm{~mL})$. The organic layer was washed with brine $(50 \mathrm{~mL})$, dried over $\mathrm{Na}_{2} \mathrm{SO}_{4}$, filtered, and concentrated under reduced pressure. Flash column chromatography $\left(\mathrm{SiO}_{2}\right.$, hexanes/EtOAc 10:1, v/v $\rightarrow$ 1:1, v/v) afforded aldehyde 10 (3.86g, $12.2 \mathrm{mmol}, 83 \%$ yield) as a colorless oil. 36: $\mathrm{R}_{\mathrm{f}}=0.70\left(\mathrm{SiO}_{2}\right.$, hexanes/EtOAc 1:1, v/v); $[\alpha]_{\mathrm{D}}^{23}=+130.0(c=1.0$, EtOAc); FTIR (film): $v_{\max } 2954,2912,2878,1740,1461,1411,1370,1296,1182,1142,1123,1099,1021,985,886$, 862, 785, $728 \mathrm{~cm}^{-1} ;{ }^{1} \mathrm{H}$ NMR (600 MHz, $\left.\mathrm{C}_{6} \mathrm{D}_{6}\right): \delta 9.68$ (brs, $\left.1 \mathrm{H}\right), 5.16$ (dd, $\left.J=5.8,3.8 \mathrm{~Hz}, 1 \mathrm{H}\right), 3.93$ (dt, $J=4.2,2.2 \mathrm{~Hz}, 1 \mathrm{H}), 3.63(\mathrm{dt}, J=4.7,2.3 \mathrm{~Hz}, 1 \mathrm{H}), 3.45(\mathrm{dd}, J=5.4,2.5 \mathrm{~Hz}, 1 \mathrm{H}), 3.24(\mathrm{~s}, 3 \mathrm{H}), 3.07$ (dd, $J=1.9,0.9 \mathrm{~Hz}, 1 \mathrm{H}), 2.32(\mathrm{ddt}, J=14.4,5.9,0.8 \mathrm{~Hz}, 1 \mathrm{H}), 2.05-1.89(\mathrm{~m}, 2 \mathrm{H}), 1.22-1.12(\mathrm{~m}, 1 \mathrm{H}), 1.02(\mathrm{t}$, $J=7.9 \mathrm{~Hz}, 9 \mathrm{H}), 0.60(\mathrm{qd}, J=7.9,2.3 \mathrm{~Hz}, 6 \mathrm{H}) \mathrm{ppm} ;{ }^{13} \mathrm{C} \mathrm{NMR}\left(151 \mathrm{MHz}, \mathrm{C}_{6} \mathrm{D}_{6}\right): \delta 202.1,105.0,82.5,76.9$, 72.5, 65.2, 55.3, 41.5, 32.3, 7.0, 5.3 ppm; HRMS (ESI-TOF) $m / z$ : $[\mathrm{M}+\mathrm{Na}]^{+}$Calcd. for $\mathrm{C}_{15} \mathrm{H}_{28} \mathrm{O}_{5} \mathrm{SiNa}^{+}$ 339.1597; Found 339.1598.

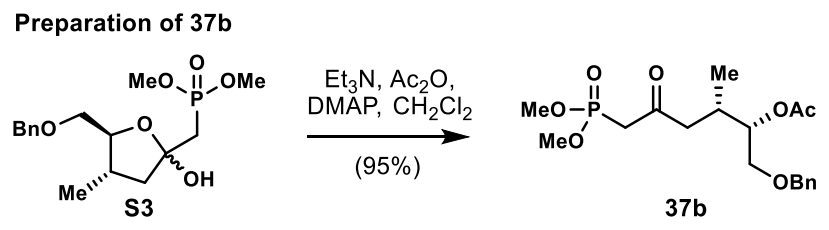

(2S,3S)-1-(Benzyloxy)-6-(dimethoxyphosphoryl)-3-methyl-5-oxohexan-2-yl acetate (37b): To a stirred

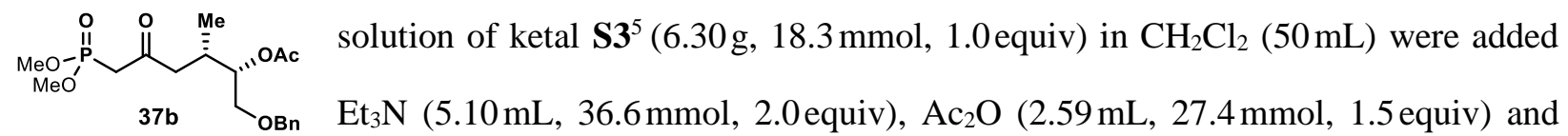
$N, N$-dimethylpyridin-4-amine (DMAP, $220 \mathrm{mg}, 1.83 \mathrm{mmol}, 0.1$ equiv) at $0{ }^{\circ} \mathrm{C}$. The resulting mixture was allowed to warm to $23^{\circ} \mathrm{C}$ and stirred for $15 \mathrm{~h}$ before it was quenched by the addition of $\mathrm{MeOH}(2 \mathrm{~mL})$ and 
a sat. aq. $\mathrm{NaHCO}_{3}$ solution $(30 \mathrm{~mL})$. The layers were separated, and the aqueous layer was extracted with $\mathrm{CH}_{2} \mathrm{Cl}_{2}(3 \times 50 \mathrm{~mL})$. The combined organic layers were washed with brine $(20 \mathrm{~mL})$, dried over $\mathrm{Na}_{2} \mathrm{SO}_{4}$, filtered, and concentrated under reduced pressure. Flash column chromatography $\left(\mathrm{SiO}_{2}\right.$, hexanes/EtOAc $1: 1, v / v \rightarrow 1: 3, v / v)$ of the residue afforded phosphonate $\mathbf{3 7 b}(8.07 \mathrm{~g}, 17.4 \mathrm{mmol}, 95 \%$ yield $)$ as a colorless oil. 37b: $\mathrm{R}_{\mathrm{f}}=0.40\left(\mathrm{SiO}_{2}, 100 \%\right.$ EtOAc $) ;[\alpha]_{\mathrm{D}}^{23}=-6.1\left(c=0.87, \mathrm{CHCl}_{3}\right)$; FT-IR (film): $v_{\max } 3643,2957,2856$, 1716, 1454, 1402, 1372, 1236, 1158, 1104, 1023, 808, 740, $699 \mathrm{~cm}^{-1} ;{ }^{1} \mathrm{H}$ NMR $\left(600 \mathrm{MHz}, \mathrm{CD}_{3} \mathrm{CN}\right): \delta 7.41-$ $7.23(\mathrm{~m}, 5 \mathrm{H}), 4.86$ (ddd, $J=6.7,5.5,4.0 \mathrm{~Hz}, 1 \mathrm{H}), 4.59-4.34(\mathrm{~m}, 2 \mathrm{H}), 3.70(\mathrm{~s}, 3 \mathrm{H}), 3.68(\mathrm{~s}, 3 \mathrm{H}), 3.58-3.51$ (m, 2H), 3.18-2.99 (m, 2H), 2.69 (dd, $J=17.7,4.2 \mathrm{~Hz}, 1 \mathrm{H}), 2.52-2.44$ (m, 1 H), 2.43-2.33 (m, $1 \mathrm{H}), 2.00$ (s, $3 \mathrm{H}), 0.89(\mathrm{~d}, J=6.8 \mathrm{~Hz}, 3 \mathrm{H}) \mathrm{ppm} ;{ }^{13} \mathrm{C} \mathrm{NMR}\left(151 \mathrm{MHz}, \mathrm{CD}_{3} \mathrm{CN}\right): \delta 202.23$ (d, J=6.1 Hz), 171.3, 139.5, 129.3, 128.6, 128.5, 76.2, 73.6, 70.3, 53.49 (d, $J=6.5 \mathrm{~Hz}), 47.43(\mathrm{~d}, J=1.9 \mathrm{~Hz}), 47.42,42.1,41.3,30.6$, 21.3, 16.7 ppm; HRMS (ESI-TOF) $m / z$ : $[\mathrm{M}+\mathrm{Na}]^{+}$Calcd. for $\mathrm{C}_{18} \mathrm{H}_{27} \mathrm{O}_{7} \mathrm{PNa}^{+}$409.1387; Found 409.1390.

Methyl (8E)-3,7-anhydro-14- $O$-benzyl-2,5,8,9,11,12-hexadeoxy-13- $O$-(4-methoxybenzyl)-12-methyl6-O-(triethylsilyl)-D-threo- $\beta$-L-galacto-tetradec-8-enofuranosid-10-ulose (38a): To a stirred solution of

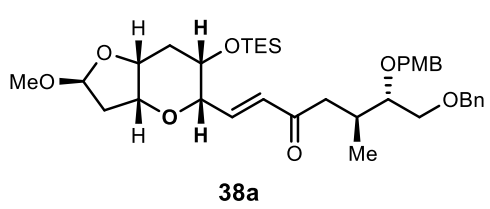
aldehyde 36 ( $1.10 \mathrm{~g}, 3.47 \mathrm{mmol}, 1.0$ equiv) and phosphonate $\mathbf{3 7 a}(2.09 \mathrm{~g}$, $4.51 \mathrm{mmol}, 1.3$ equiv) in $\mathrm{MeCN}(30 \mathrm{~mL})$ at $0^{\circ} \mathrm{C}$ was added $\mathrm{LiCl}(441 \mathrm{mg}$, $10.4 \mathrm{mmol}, 3.0$ equiv) and $\mathrm{Et}_{3} \mathrm{~N}(2.42 \mathrm{~mL}, 17.3 \mathrm{mmol}, 5.0$ equiv). The resulting mixture was allowed to warm to $23^{\circ} \mathrm{C}$ and stirred for $1.5 \mathrm{~h}$ before it was quenched by the addition of sat. aq. $\mathrm{NH}_{4} \mathrm{Cl}$ solution $(20 \mathrm{~mL})$. The layers were separated, and the aqueous layer was extracted with EtOAc $(3 \times 50 \mathrm{~mL})$. The combined organic layers were washed with brine $(30 \mathrm{~mL})$, dried over $\mathrm{Na}_{2} \mathrm{SO}_{4}$, filtered, and concentrated under reduced pressure. Flash column chromatography $\left(\mathrm{SiO}_{2}\right.$, hexanes/EtOAc $10: 1, v / v \rightarrow 2: 1, v / v)$ of the residue afforded enone $38 \mathbf{a}(1.93 \mathrm{~g}, 2.95 \mathrm{mmol}, 85 \%$ yield $)$ as a colorless oil. 38a: $\mathrm{R}_{\mathrm{f}}=0.70\left(\mathrm{SiO}_{2}\right.$, hexanes/EtOAc $\left.2: 1, v / v\right) ;[\alpha]_{\mathrm{D}}^{23}=+30.7\left(c=0.40\right.$, EtOAc); FT-IR (film): $v_{\max } 2954$, 2910, 2876, 1696, 1637, 1613, 1513, 1455, 1368, 1301, 1247, 1180, 1140, 1097, 1054, 1028, 983, 889, 820, $740,699 \mathrm{~cm}^{-1} ;{ }^{1} \mathrm{H}$ NMR $\left(600 \mathrm{MHz}, \mathrm{C}_{6} \mathrm{D}_{6}\right): \delta 7.34-7.17(\mathrm{~m}, 5 \mathrm{H}), 7.13-7.00(\mathrm{~m}, 2 \mathrm{H}), 6.86(\mathrm{~d}, J=4.6 \mathrm{~Hz}$, $1 \mathrm{H}), 6.84-6.78(\mathrm{~m}, 2 \mathrm{H}), 6.50(\mathrm{dd}, J=16.0,1.5 \mathrm{~Hz}, 1 \mathrm{H}), 5.18(\mathrm{dd}, J=5.8,3.8 \mathrm{~Hz}, 1 \mathrm{H}), 4.66(\mathrm{~d}, J=11.3 \mathrm{~Hz}$, $1 \mathrm{H}), 4.46(\mathrm{~d}, J=11.3 \mathrm{~Hz}, 1 \mathrm{H}), 4.33(\mathrm{~s}, 2 \mathrm{H}), 3.75-3.69(\mathrm{~m}, 1 \mathrm{H}), 3.59(\mathrm{dd}, J=5.5,2.6 \mathrm{~Hz}, 1 \mathrm{H}), 3.53-3.44$ 
(m, 3 H), 3.43-3.37 (m, 2 H), $3.32(\mathrm{~s}, 3 \mathrm{H}), 3.26(\mathrm{~s}, 3 \mathrm{H}), 2.82(\mathrm{dd}, J=16.6,4.3 \mathrm{~Hz}, 1 \mathrm{H}), 2.75-2.64(\mathrm{~m}, 1 \mathrm{H})$, 2.47 (dd, $J=16.6,8.8 \mathrm{~Hz}, 1 \mathrm{H}), 2.33$ (dd, $J=14.3,5.8 \mathrm{~Hz}, 1 \mathrm{H}), 2.09$ (d, $J=15.1 \mathrm{~Hz}, 1 \mathrm{H}), 2.04$ (ddd, $J=14.3$, $5.5,3.8 \mathrm{~Hz}, 1 \mathrm{H}), 1.34(\mathrm{dt}, J=15.2,4.4 \mathrm{~Hz}, 1 \mathrm{H}), 1.09-0.85(\mathrm{~m}, 12 \mathrm{H}), 0.62-0.55(\mathrm{~m}, 6 \mathrm{H}) . \mathrm{ppm} ;{ }^{13} \mathrm{C}$ NMR $\left(151 \mathrm{MHz}, \mathrm{C}_{6} \mathrm{D}_{6}\right): \delta 198.5,159.6,143.5,139.2,131.8,130.5,129.6,128.6,127.79,127.78,127.69,114.0$, $105.1,81.7,77.7,77.1,73.4,72.5,72.5,71.8,66.1,55.3,54.8,43.7,41.7,33.0,31.8,17.1,7.1,5.4$ ppm; HRMS (ESI-TOF) $m / z$ : [M+Na] $]^{+}$Calcd. for $\mathrm{C}_{37} \mathrm{H}_{54} \mathrm{O}_{8} \mathrm{SiNa}^{+}$677.3480; Found 677.3479.

\section{Methyl (8E)-13-O-acetyl-3,7-anhydro-14- $O$-benzyl-2,5,8,9,11,12-hexadeoxy-12-methyl-6-O-(triethyl-}

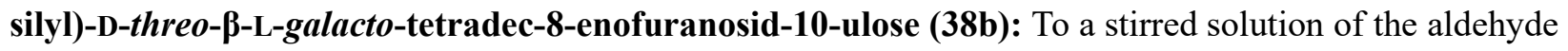

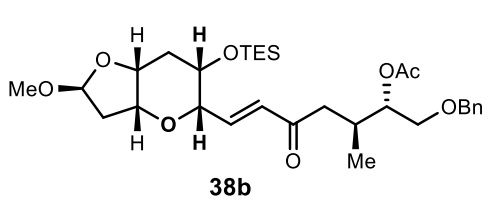
$36(3.10 \mathrm{~g}, 9.80 \mathrm{mmol}, 1.0$ equiv) and phosphonate $\mathbf{3 7 b}(4.92 \mathrm{~g}$, $12.7 \mathrm{mmol}, 1.3$ equiv) in $\mathrm{MeCN}(50 \mathrm{~mL})$ at $0^{\circ} \mathrm{C}$ were added $\mathrm{LiCl}(1.25 \mathrm{~g}$, $29.4 \mathrm{mmol}, 3.0$ equiv) and $\mathrm{Et}_{3} \mathrm{~N}(6.83 \mathrm{~mL}, 49.0 \mathrm{mmol}, 5.0$ equiv). The resulting mixture was allowed to warm to $23^{\circ} \mathrm{C}$ and stirred for $1.5 \mathrm{~h}$ before it was quenched by the addition of sat. aq. $\mathrm{NH}_{4} \mathrm{Cl}$ solution $(50 \mathrm{~mL})$. The layers were separated, and the aqueous layer was extracted with EtOAc $(3 \times 50 \mathrm{~mL})$. The combined organic layers were washed with brine $(20 \mathrm{~mL})$, dried over $\mathrm{Na}_{2} \mathrm{SO}_{4}$, filtered, and concentrated under reduced pressure. Flash column chromatography $\left(\mathrm{SiO}_{2}\right.$, hexanes/EtOAc $10: 1, v / v \rightarrow 2: 1, v / v)$ of the residue afforded enone $\mathbf{3 8 b}(5.03 \mathrm{~g}, 8.72 \mathrm{mmol}, 89 \%$ yield $)$ as a colorless oil. 38b: $\mathrm{R}_{\mathrm{f}}=0.60\left(\mathrm{SiO}_{2}\right.$, hexanes/EtOAc $\left.2: 1, v / v\right) ;[\alpha]_{\mathrm{D}}^{23}=+30.3\left(c=2.0\right.$, EtOAc); FT-IR (film): $v_{\max } 2953,2910$, 2876, 1740, 1697, 1675, 1637, 1455, 1415, 1371, 1238, 1181, 1139, 1127, 1097, 1053 1023, 982, 887, 739, $699 \mathrm{~cm}^{-1} ;{ }^{1} \mathrm{H}$ NMR $\left(600 \mathrm{MHz}, \mathrm{C}_{6} \mathrm{D}_{6}\right): \delta 7.35-7.27(\mathrm{~m}, 4 \mathrm{H}), 7.23-7.13(\mathrm{~m}, 1 \mathrm{H}), 6.76(\mathrm{dd}, J=16.0,5.0 \mathrm{~Hz}$, $1 \mathrm{H}), 6.31(\mathrm{dd}, J=16.0,1.6 \mathrm{~Hz}, 1 \mathrm{H}), 5.19(\mathrm{dd}, J=5.7,3.9 \mathrm{~Hz}, 1 \mathrm{H}), 4.95(\mathrm{ddd}, J=6.4,5.4,4.4 \mathrm{~Hz}, 1 \mathrm{H}), 4.61-$ 4.37 (m, 2 H), 4.09 (dd, $J=5.4,2.7 \mathrm{~Hz}, 1 \mathrm{H}), 3.99-3.96(\mathrm{~m}, 1 \mathrm{H}), 3.94(\mathrm{dt}, J=5.1,1.9 \mathrm{~Hz}, 1 \mathrm{H}), 3.84(\mathrm{td}$, $J=3.6,2.0 \mathrm{~Hz}, 1 \mathrm{H}), 3.58-3.49(\mathrm{~m}, 2 \mathrm{H}), 3.36(\mathrm{~s}, 3 \mathrm{H}), 2.66(\mathrm{dd}, J=16.6,3.7 \mathrm{~Hz}, 1 \mathrm{H}), 2.55$ (ddq, $J=13.2$, 6.6, 3.6Hz, $1 \mathrm{H}), 2.42(\mathrm{dd}, J=16.6,9.5 \mathrm{~Hz}, 1 \mathrm{H}), 2.38(\mathrm{dd}, J=14.5,5.8 \mathrm{~Hz}, 1 \mathrm{H}), 2.24$ (dt, $J=15.1,3.0 \mathrm{~Hz}$, $1 \mathrm{H}), 2.09-2.03(\mathrm{~m}, 4 \mathrm{H}), 1.93$ (dt, $J=15.1,4.4 \mathrm{~Hz}, 1 \mathrm{H}), 0.93$ (t, $J=7.9 \mathrm{~Hz}, 9 \mathrm{H}), 0.89$ (d, $J=6.8 \mathrm{~Hz}, 3 \mathrm{H})$, 0.59-0.55 (m, 6H) ppm; ${ }^{13} \mathrm{C}$ NMR (151 MHz, $\left.\mathrm{C}_{6} \mathrm{D}_{6}\right): \delta 198.8,170.8,143.9,138.1,130.2,128.5,127.8$, 127.7, 104.8, 77.8, 77.1, 75.8, 73.2, 72.6, 69.5, 66.0, 55.6, 43.0, 41.2, 32.9, 30.1, 21.2, 16.7, 6.9, 5.0 ppm; 
HRMS (ESI-TOF) $m / z$ : [M+Na $]^{+}$Calcd. for $\mathrm{C}_{31} \mathrm{H}_{48} \mathrm{O}_{8} \mathrm{SiNa}^{+}$599.3011; Found 599.3009.

Methyl 3,7-anhydro-14-O-benzyl-2,5,8,9,11,12-hexadeoxy-13-O-(4-methoxybenzyl)-8,12-dimethyl-6O-(triethylsilyl)-D-arabino- $\beta$-L-galacto-tetradecofuranosid-10-ulose (39a): To a stirred solution of

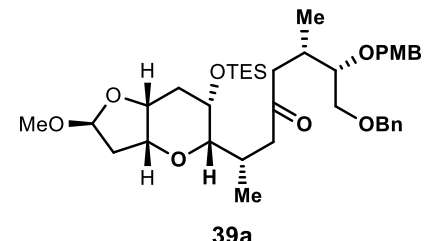

$39 a$

$\mathrm{CuCN}$ (349 mg, $3.90 \mathrm{mmol}, 3.0$ equiv) in THF (50 mL) was added MeLi (1.6 M in $\mathrm{Et}_{2} \mathrm{O}, 4.87 \mathrm{~mL}, 7.80 \mathrm{mmol}, 6.0$ equiv) at $-78^{\circ} \mathrm{C}$. The resulting mixture was stirred at $-40^{\circ} \mathrm{C}$ for $10 \mathrm{~min}$ and then cooled to $-78^{\circ} \mathrm{C}$. Then, $\mathrm{TMSCl}(990 \mu \mathrm{L}$, $7.80 \mathrm{mmol}, 6.0$ equiv) and 38a $(850 \mathrm{mg}, 1.30 \mathrm{mmol}, 1.0$ equiv) in THF $(10 \mathrm{~mL})$ were added. The resulting mixture was allowed to warm to $-40^{\circ} \mathrm{C}$ and stirred for additional $0.5 \mathrm{~h}$ before it was quenched by the addition of sat. aq. $\mathrm{NH}_{4} \mathrm{Cl}$ solution $(30 \mathrm{~mL})$. The mixture was allowed to warm to $23^{\circ} \mathrm{C}$ and vigorously stirred for $3 \mathrm{~h}$. The layers were separated and filtered through a pad of celite, and the aqueous layer was extracted with EtOAc $(3 \times 30 \mathrm{~mL})$. The combined organic layers were washed with aq. $\mathrm{HCl}(0.05 \mathrm{M}, 30 \mathrm{~mL})$, $\mathrm{NaHCO}_{3}\left(30 \mathrm{~mL}\right.$, sat. aq.), dried over $\mathrm{Na}_{2} \mathrm{SO}_{4}$, filtered, and concentrated under reduced pressure. Flash column chromatography $\left(\mathrm{SiO}_{2}\right.$, hexanes/EtOAc 10:1, v/v $\left.\rightarrow 2: 1, v / v\right)$ of the residue afforded ketone 39a (706 mg, $1.05 \mathrm{mmol}, 81 \%$ yield) as a colorless oil. 39a: $\mathrm{R}_{\mathrm{f}}=0.80\left(\mathrm{SiO}_{2}\right.$, hexanes/EtOAc 2:1, v/v); $[\alpha]_{\mathrm{D}}^{23}=+14.8\left(c=0.50\right.$, EtOAc); FT-IR (film): $v_{\max } 2953,2910,2876,1709,1613,1514,1456,1414,1369$, 1302, 1248, 1183, 1139, 1098, 1029, 972, 943, 824, $738 \mathrm{~cm}^{-1} ;{ }^{1} \mathrm{H}$ NMR $\left(600 \mathrm{MHz}, \mathrm{C}_{6} \mathrm{D}_{6}\right): \delta 7.36-7.18$ (m, 5H), 7.13-6.97 (m, 2 H), $6.83(\mathrm{~d}, J=8.6 \mathrm{~Hz}, 2 \mathrm{H}), 5.18(\mathrm{dd}, J=5.8,3.9 \mathrm{~Hz}, 1 \mathrm{H}), 4.68(\mathrm{~d}, J=11.3 \mathrm{~Hz}, 1 \mathrm{H})$, $4.47(\mathrm{~d}, J=11.3 \mathrm{~Hz}, 1 \mathrm{H}), 4.35(\mathrm{~d}, J=1.7 \mathrm{~Hz}, 2 \mathrm{H}), 3.73(\mathrm{dt}, J=4.6,2.0 \mathrm{~Hz}, 1 \mathrm{H}), 3.63$ (brs, $1 \mathrm{H}), 3.58$ (dd, $J=5.4,2.5 \mathrm{~Hz}, 1 \mathrm{H}), 3.53-3.46(\mathrm{~m}, 2 \mathrm{H}), 3.45(\mathrm{td}, J=5.3,4.0 \mathrm{~Hz}, 1 \mathrm{H}), 3.32(\mathrm{~s}, 3 \mathrm{H}), 3.27(\mathrm{~s}, 3 \mathrm{H}), 2.74$ (dd, $J=16.1,4.0 \mathrm{~Hz}, 1 \mathrm{H}), 2.68-2.52(\mathrm{~m}, 4 \mathrm{H}), 2.33-2.12(\mathrm{~m}, 4 \mathrm{H}), 2.02(\mathrm{ddd}, J=14.2,5.4,3.8 \mathrm{~Hz}, 1 \mathrm{H}), 1.31$ (dt, $J=15.3,4.4 \mathrm{~Hz}, 1 \mathrm{H}), 1.09(\mathrm{t}, J=7.9 \mathrm{~Hz}, 9 \mathrm{H}), 1.00(\mathrm{~d}, J=6.6 \mathrm{~Hz}, 3 \mathrm{H}), 0.92(\mathrm{~d}, J=6.5 \mathrm{~Hz}, 3 \mathrm{H}), 0.75-0.60$ (m, 6H).ppm; ${ }^{13} \mathrm{C}$ NMR (151 MHz, $\left.\mathrm{C}_{6} \mathrm{D}_{6}\right): \delta 209.0,159.7,139.2,131.8,129.7,128.6,127.8,127.7,114.0$, $105.1,81.9,81.8,77.6,73.4,72.9,72.5,71.8,63.5,55.2,54.8,47.1,46.1,41.8,33.4,31.6,30.7,17.1,16.5$, 7.3, 5.7 ppm; HRMS (ESI-TOF) $\mathrm{m} / z$ : $[\mathrm{M}+\mathrm{Na}]^{+}$Calcd. for $\mathrm{C}_{38} \mathrm{H}_{58} \mathrm{O}_{8} \mathrm{SiNa}^{+}$693.3793; Found 693.3794. 
Methyl 13-O-acetyl-3,7-anhydro-14- $O$-benzyl-2,5,8,9,11,12-hexadeoxy-8,12-dimethyl-6- $O$-(triethylsilyl)-D-arabino-p-L-galacto-tetradecofuranosid-10-ulose (39b): To a stirred solution of CuCN (605 mg,

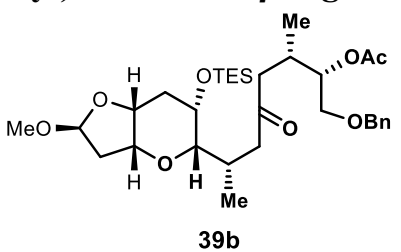

$6.75 \mathrm{mmol}, 3.0$ equiv) in THF $(60 \mathrm{~mL})$ was added $\mathrm{MeLi}\left(1.6 \mathrm{M}\right.$ in $\mathrm{Et}_{2} \mathrm{O}, 8.44 \mathrm{~mL}$, $13.5 \mathrm{mmol}, 6.0$ equiv) at $-78^{\circ} \mathrm{C}$. The reaction mixture was stirred at $-40^{\circ} \mathrm{C}$ for $10 \mathrm{~min}$ and then cooled to $-78^{\circ} \mathrm{C}$. Then, TMSCl $(1.71 \mathrm{~mL}, 13.5 \mathrm{mmol}$, 6.0 equiv) and 38a $(1.30 \mathrm{~g}, 2.25 \mathrm{mmol}, 1.0$ equiv) in THF $(15 \mathrm{~mL})$ were added. The resulting mixture was allowed to warm to $-40^{\circ} \mathrm{C}$ and stirred for additional $0.5 \mathrm{~h}$ before it was quenched by the addition of sat. aq. $\mathrm{NH}_{4} \mathrm{Cl}$ solution $(40 \mathrm{~mL})$. The mixture was allowed to warm to $23^{\circ} \mathrm{C}$ and vigorously stirred for $3 \mathrm{~h}$. The layers were separated and filtered through a pad of celite, and the aqueous layer was extracted with EtOAc $(3 \times 30 \mathrm{~mL})$. The combined organic layers were washed with aq. $\mathrm{HCl}(0.05 \mathrm{M}, 30 \mathrm{~mL}), \mathrm{NaHCO}_{3}(30 \mathrm{~mL}$, sat. aq.), dried over $\mathrm{Na}_{2} \mathrm{SO}_{4}$, filtered, and concentrated under reduced pressure. Flash column chromatography $\left(\mathrm{SiO}_{2}\right.$, hexanes/EtOAc 10:1, v/v $\left.\rightarrow 2: 1, v / v\right)$ of the residue afforded ketone 39b $(1.11 \mathrm{~g}, 1.87 \mathrm{mmol}, 83 \%$ yield) as a colorless oil. 39b: $\mathrm{R}_{\mathrm{f}}=0.60\left(\mathrm{SiO}_{2}\right.$, hexanes/EtOAc $\left.2: 1, v / v\right) ;[\alpha]_{\mathrm{D}}^{23}=+27.5(c=0.50$, EtOAc); FTIR (film): $v_{\max } 2954,2912,2877,1740,1712,1455,1415,1372,1311,1238,1185,1139,1099,1060,1027$, 972, 943, 880, 789, 737, $698 \mathrm{~cm}^{-1} ;{ }^{1} \mathrm{H}$ NMR $\left(600 \mathrm{MHz}, \mathrm{CDCl}_{3}\right): \delta 7.36-7.27(\mathrm{~m}, 5 \mathrm{H}), 5.16-5.12(\mathrm{~m}, 1 \mathrm{H})$, $4.92(\mathrm{q}, J=5.4 \mathrm{~Hz}, 1 \mathrm{H}), 4.58-4.45(\mathrm{~m}, 2 \mathrm{H}), 3.91$ (ddd, $J=10.6,5.1,2.5 \mathrm{~Hz}, 2 \mathrm{H}), 3.84(\mathrm{~s}, 1 \mathrm{H}), 3.57-3.50$ (m, $2 \mathrm{H}), 3.34(\mathrm{~s}, 3 \mathrm{H}), 2.81(\mathrm{~d}, J=9.5 \mathrm{~Hz}, 1 \mathrm{H}), 2.71(\mathrm{dd}, J=15.9,4.5 \mathrm{~Hz}, 1 \mathrm{H}), 2.54-2.43(\mathrm{~m}, 2 \mathrm{H}), 2.41-$ $2.33(\mathrm{~m}, 1 \mathrm{H}), 2.31-2.17(\mathrm{~m}, 4 \mathrm{H}), 2.06(\mathrm{~s}, 3 \mathrm{H}), 1.95(\mathrm{dt}, J=14.3,4.7 \mathrm{~Hz}, 1 \mathrm{H}), 1.78$ (dt, J=15.4, 4.4 Hz, $1 \mathrm{H}), 0.98(\mathrm{t}, J=7.9 \mathrm{~Hz}, 9 \mathrm{H}), 0.89(\mathrm{~d}, J=6.7 \mathrm{~Hz}, 3 \mathrm{H}), 0.86(\mathrm{~d}, J=6.7 \mathrm{~Hz}, 3 \mathrm{H}), 0.67-0.60(\mathrm{~m}, 6 \mathrm{H}) \mathrm{ppm} ;{ }^{13} \mathrm{C}$ NMR $\left(151 \mathrm{MHz}, \mathrm{CDCl}_{3}\right): \delta 209.8,170.8,138.1,128.5,127.8,127.7,104.8,82.2,77.7,75.9,73.2,72.8$, 69.6, 63.1 , 55.5, 47.3, 45.6, 41.2, 33.4, 30.5, 30.0, 21.3, 16.7, 16.3, 7.0, 5.3 ppm; HRMS (ESI-TOF) $m / z$ : $[\mathrm{M}+\mathrm{Na}]^{+}$Calcd. for $\mathrm{C}_{32} \mathrm{H}_{52} \mathrm{O}_{8} \mathrm{SiNa}^{+}$615.3324; Found 615.3332. 
Methyl (14S)-8,12-anhydro-3,4,6,7,10,13-hexadeoxy-2-O-(4-methoxybenzyl)-3,7-dimethyl-9-O(triethylsilyl)-D-lyxo-D-manno-tetradecodialdo-14,11-furanosid-5-ulose (32a): A solution of benzyl

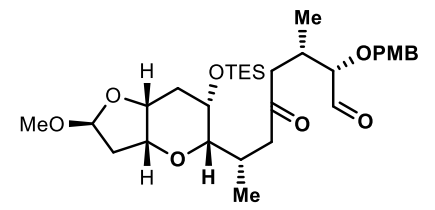

32a: fragment $A$

ether 12 (30.7 mg, $0.0457 \mathrm{mmol}, 1.0$ equiv) and excess Raney Ni ( $200 \mathrm{mg})$ in $\mathrm{EtOH}(3 \mathrm{~mL})$ was stirred under a hydrogen atmosphere $(1 \mathrm{bar})$ at $23^{\circ} \mathrm{C}$ for $12 \mathrm{~h}$. The resulting mixture was filtrated through a pad of Celite and the filtrate was concentrated under reduced pressure to give the crude alcohol. Flash column chromatography $\left(\mathrm{SiO}_{2}\right.$, hexanes/EtOAc 10:1, v/v $\rightarrow 5: 1, v / v)$ of the residue afforded the corresponding alcohol intermediate (14.1 mg, $0.0242 \mathrm{mmol}, 53 \%$ yield), which was used for the next step without further characterization (Note: the yield of the reaction was $39 \%$ at $350 \mathrm{mg}$ scale).

To a stirred solution of the above alcohol $\left(14.1 \mathrm{mg}, 0.0242 \mathrm{mmol}, 1.0\right.$ equiv) in $\mathrm{CH}_{2} \mathrm{Cl}_{2}(2 \mathrm{~mL})$ were added $\mathrm{NaHCO}_{3}(14.2 \mathrm{mg}, 0.169 \mathrm{mmol}, 7.0$ equiv) and Dess-Martin periodinane $(30.8 \mathrm{mg}, 0.0726 \mathrm{mmol}$, 3.0 equiv) at $0{ }^{\circ} \mathrm{C}$. The reaction mixture was stirred for $1 \mathrm{~h}$ at $23^{\circ} \mathrm{C}$ before it was quenched by the addition of sat. aq. $\mathrm{Na}_{2} \mathrm{~S}_{2} \mathrm{O}_{3}$ solution $(5 \mathrm{~mL})$. The layers were separated, and the aqueous layer was extracted with $\mathrm{CH}_{2} \mathrm{Cl}_{2}(3 \times 10 \mathrm{~mL})$. The organic layer was washed with brine $(10 \mathrm{~mL})$, dried over $\mathrm{Na}_{2} \mathrm{SO}_{4}$, filtered, and concentrated under reduced pressure. Flash column chromatography $\left(\mathrm{SiO}_{2}\right.$, hexanes/EtOAc 10:1, v/v $\rightarrow$ 1:1, $v / v)$ of the residue afforded aldehyde fragment $A(\mathbf{A})(11.3 \mathrm{mg}, 0.0196 \mathrm{mmol}, 81 \%$ yield $)$ as a colorless oil. 32a: $\mathrm{R}_{\mathrm{f}}=0.50\left(\mathrm{SiO}_{2}\right.$, hexanes/EtOAc $\left.2: 1, v / v\right) ;[\alpha]_{\mathrm{D}}^{23}=+3.3\left(c=0.20, \mathrm{CHCl}_{3}\right)$; FT-IR (film): $v_{\max } 2954,1877$, $1731,1711,1613,1514,1463,1373,1303,1249,1183,1249,1141,1098,1029,973,742,726 \mathrm{~cm}^{-1} ;{ }^{1} \mathrm{H}$ NMR $\left(600 \mathrm{MHz}, \mathrm{C}_{6} \mathrm{D}_{6}\right): \delta 9.43(\mathrm{~d}, J=2.0 \mathrm{~Hz}, 1 \mathrm{H}), 7.15(\mathrm{~d}, J=8.6 \mathrm{~Hz}, 2 \mathrm{H}$, one proton merged in solvent peak), $6.78(\mathrm{~d}, J=8.6 \mathrm{~Hz}, 2 \mathrm{H}), 5.19(\mathrm{dd}, J=5.8,3.9 \mathrm{~Hz}, 1 \mathrm{H}), 4.43(\mathrm{~d}, J=11.4 \mathrm{~Hz}, 1 \mathrm{H}), 4.18(\mathrm{~d}, J=11.4 \mathrm{~Hz}$, $1 \mathrm{H}), 3.72(\mathrm{dt}, J=4.7,2.3 \mathrm{~Hz}, 1 \mathrm{H}), 3.61(\mathrm{~s}, 1 \mathrm{H}), 3.59(\mathrm{dd}, J=5.4,2.5 \mathrm{~Hz}, 1 \mathrm{H}), 3.36(\mathrm{dd}, J=4.9,2.1 \mathrm{~Hz}, 1 \mathrm{H})$, $3.30(\mathrm{~s}, 3 \mathrm{H}), 3.28(\mathrm{~s}, 3 \mathrm{H}), 2.66-2.49(\mathrm{~m}, 5 \mathrm{H}), 2.29(\mathrm{dd}, J=14.2,5.8 \mathrm{~Hz}, 1 \mathrm{H}), 2.18-2.07$ (m, $3 \mathrm{H}), 2.03$ (ddd, $J=14.2,5.5,3.9 \mathrm{~Hz}, 1 \mathrm{H}), 1.31(\mathrm{dt}, J=15.3,4.4 \mathrm{~Hz}, 1 \mathrm{H}), 1.08(\mathrm{t}, J=7.9 \mathrm{~Hz}, 9 \mathrm{H}), 0.90(\mathrm{~d}, J=6.7 \mathrm{~Hz}$, 6H), 0.74-0.60 (m, 6H) ppm; ${ }^{13} \mathrm{C} \mathrm{NMR}\left(151 \mathrm{MHz}, \mathrm{C}_{6} \mathrm{D}_{6}\right): \delta 208.3,202.6,160.0,130.2,130.0,114.2,105.1$, 86.6, 81.8, 77.6, 72.9, 72.5, 63.4, 55.2, 54.8, 47.1, 44.8, 41.8, 33.3, 30.6, 30.2, 16.82, 16.45, 7.2, 5.7 ppm; HRMS (ESI-TOF) $m / z$ : $[\mathrm{M}+\mathrm{Na}]^{+}$Calcd. for $\mathrm{C}_{31} \mathrm{H}_{50} \mathrm{O}_{8} \mathrm{SiNa}^{+}$601.3167; Found 601.3162 . 
Methyl (14S)-2-O-acetyl-8,12-anhydro-3,4,6,7,10,13-hexadeoxy-3,7-dimethyl-9-O-(triethylsilyl)-Dlyxo-D-manno-tetradecodialdo-14,11-furanosid-5-ulose (32b): A solution of benzyl ether 39b (2.30g,

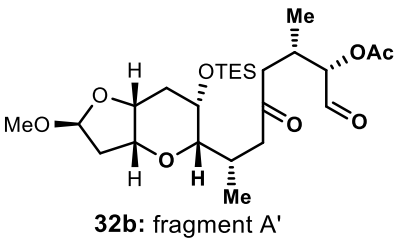

$3.88 \mathrm{mmol}, 1.0$ equiv) and $\mathrm{Pd} / \mathrm{C}(30 \mathrm{wt} \% \mathrm{Pd} / \mathrm{C}, 20 \%$ loading of 39b, $460 \mathrm{mg})$ in THF $(50 \mathrm{~mL})$ was stirred under a hydrogen atmosphere $(1 \mathrm{bar})$ at $23^{\circ} \mathrm{C}$ for $1.5 \mathrm{~h}$. The resulting mixture was filtered through a pad of Celite and the filtrate was concentrated under reduced pressure to give the crude alcohol which was used for the next step without further purification.

To a stirred solution of the above alcohol in $\mathrm{CH}_{2} \mathrm{Cl}_{2}(50 \mathrm{~mL})$ were added $\mathrm{NaHCO}_{3}(2.28 \mathrm{~g}, 27.2 \mathrm{mmol}$, 7.0 equiv) and Dess-Martin periodinane ( $4.11 \mathrm{~g}, 9.70 \mathrm{mmol}, 2.5$ equiv). The resulting mixture was allowed to warm to $23^{\circ} \mathrm{C}$ and stirred for $1 \mathrm{~h}$ before it was quenched by the addition of sat. aq. $\mathrm{NaHCO}_{3}$ solution $(30 \mathrm{~mL})$ and sat. aq. $\mathrm{Na}_{2} \mathrm{~S}_{2} \mathrm{O}_{3}$ solution $(50 \mathrm{~mL})$. The layers were separated, and the aqueous layer was extracted with $\mathrm{CH}_{2} \mathrm{Cl}_{2}(3 \times 50 \mathrm{~mL})$. The organic layer was washed with brine $(50 \mathrm{~mL})$, dried over $\mathrm{Na}_{2} \mathrm{SO}_{4}$, filtered, and concentrated under reduced pressure. Flash column chromatography $\left(\mathrm{SiO}_{2}\right.$, hexanes/EtOAc $10: 1, v / v \rightarrow 1: 1, v / v)$ afforded fragment $A^{\prime}(\mathbf{3 2 b} ; 1.26 \mathrm{~g}, 2.52 \mathrm{mmol}, 65 \%$ yield for the two steps) as a colorless oil. 32b: $\mathrm{R}_{\mathrm{f}}=0.50\left(\mathrm{SiO}_{2}\right.$, hexanes/EtOAc 2:1, v/v); $[\alpha]_{\mathrm{D}}^{23}=+19.5(c=0.62$, EtOAc); FT-IR (film): $v_{\max } 2954,2912,2878,1738,1712,1459,1416,1371,1312,1231,1185,1141,1098,1059,1028,973,943$, 881, 789, 741, $726 \mathrm{~cm}^{-1} ;{ }^{1} \mathrm{H}$ NMR (600 MHz, $\left.\mathrm{C}_{6} \mathrm{D}_{6}\right): \delta 9.16(\mathrm{~s}, 1 \mathrm{H}), 5.20(\mathrm{dd}, J=5.8,3.8 \mathrm{~Hz}, 1 \mathrm{H}), 4.77$ (d, $J=3.8 \mathrm{~Hz}, 1 \mathrm{H}), 3.73(\mathrm{dd}, J=4.9,2.5 \mathrm{~Hz}, 1 \mathrm{H}), 3.60(\mathrm{dd}, J=5.5,2.5 \mathrm{~Hz}, 2 \mathrm{H}), 3.28(\mathrm{~s}, 3 \mathrm{H}), 2.69-2.58(\mathrm{~m}$, 2H), 2.57-2.45 (m, 2 H), $2.36(\mathrm{dd}, J=17.7,5.5 \mathrm{~Hz}, 1 \mathrm{H}), 2.28(\mathrm{dd}, J=14.2,5.8 \mathrm{~Hz}, 1 \mathrm{H}), 2.14(\mathrm{dd}, J=15.3$, $2.4 \mathrm{~Hz}, 1 \mathrm{H}), 2.10-1.99(\mathrm{~m}, 3 \mathrm{H}), 1.69(\mathrm{~s}, 3 \mathrm{H}), 1.30(\mathrm{dt}, J=15.3,4.3 \mathrm{~Hz}, 1 \mathrm{H}), 1.08(\mathrm{t}, J=7.9 \mathrm{~Hz}, 9 \mathrm{H}), 0.87$ $(\mathrm{d}, J=6.4 \mathrm{~Hz}, 3 \mathrm{H}), 0.81(\mathrm{~d}, J=6.8 \mathrm{~Hz}, 3 \mathrm{H}), 0.68-0.62(\mathrm{~m}, 6 \mathrm{H}) \mathrm{ppm} ;{ }^{13} \mathrm{C} \mathrm{NMR}\left(151 \mathrm{MHz}, \mathrm{C}_{6} \mathrm{D}_{6}\right): \delta 207.6$, 197.1, 169.7, 128.6, 105.1, 81.9, 81.5, 77.6, 72.8, 63.4, 55.3, 47.1, 44.2, 41.8, 33.2, 30.6, 28.7, 20.0, 17.0, 16.4, 7.2, 5.6ppm; HRMS (ESI-TOF) $m / z$ : $[\mathrm{M}+\mathrm{Na}]^{+}$Calcd. for $\mathrm{C}_{25} \mathrm{H}_{44} \mathrm{O}_{8} \mathrm{SiNa}^{+}$523.2698; Found 523.2703. 
$\left(2 R, 2^{\prime} S, 3 a^{\prime} R, 4 S, 4 a^{\prime} S, 5^{\prime} S, 8 a^{\prime} S, 9 a^{\prime} R\right)-5$-[(Benzyloxy)methyl]-2'-methoxy-4,5'-dimethyldecahydro2'H,3H-spiro[furan-2,7'-furo[3,2-b]pyrano[2,3-e]pyran] (40): To a stirred solution of 39b (35.7 mg,
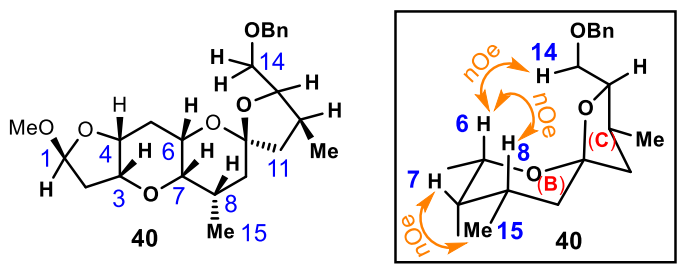
$0.0602 \mathrm{mmol}, 1.0$ equiv) in $\mathrm{MeCN}(1 \mathrm{~mL})$ at $0{ }^{\circ} \mathrm{C}$ was added HF·py (70\% HF, $200 \mu \mathrm{L}$, excess). The resulting mixture was stirred for $0.5 \mathrm{~h}$ before it was quenched by the addition of sat. aq. $\mathrm{NaHCO}_{3}$ solution $(15 \mathrm{~mL})$. The layers were separated, and the aqueous layer was extracted with EtOAc $(3 \times 15 \mathrm{~mL})$. The combined organic layers were washed with brine $(15 \mathrm{~mL})$, dried over $\mathrm{Na}_{2} \mathrm{SO}_{4}$, filtered, and concentrated under reduced pressure to give crude alcohol without further purification.

To the aboved obtained alcohol in $\mathrm{MeOH}(2 \mathrm{~mL})$ at $0{ }^{\circ} \mathrm{C}$ was added $\mathrm{K}_{2} \mathrm{CO}_{3}(41.5 \mathrm{mg}, 0.301 \mathrm{mmol}$, 5.0 equiv). The resulting mixture was stirred for $2.5 \mathrm{~h}$ before it was quenched by the addition of sat. aq. $\mathrm{NH}_{4} \mathrm{Cl}$ solution $(15 \mathrm{~mL})$. The layers were separated, and the aqueous layer was extracted with EtOAc $(3 \times 15 \mathrm{~mL})$. The combined organic layers were washed with brine $(15 \mathrm{~mL})$, dried over $\mathrm{Na}_{2} \mathrm{SO}_{4}$, filtered, and concentrated under reduced pressure to give crude alcohol without further purification.

To the aboved obtained alcohol in $\mathrm{CH}_{2} \mathrm{Cl}_{2}(2 \mathrm{~mL})$ at $23^{\circ} \mathrm{C}$ was added $p$ - $\mathrm{TsOH} \cdot \mathrm{H}_{2} \mathrm{O}(11.4 \mathrm{mg}$, $0.0602 \mathrm{mmol}, 1.0$ equiv). The resulting mixture was stirred for $1 \mathrm{~h}$ before it was quenched by the addition of sat. aq. $\mathrm{NaHCO}_{3}$ solution $(15 \mathrm{~mL})$. The layers were separated, and the aqueous layer was extracted with EtOAc $(3 \times 15 \mathrm{~mL})$. The combined organic layers were washed with brine $(15 \mathrm{~mL})$, dried over $\mathrm{Na}_{2} \mathrm{SO}_{4}$, filtered, and concentrated under reduced pressure. Flash column chromatography $\left(\mathrm{SiO}_{2}\right.$, hexanes/EtOAc $10: 1, v / v \rightarrow 3: 1, v / v)$ of the residue afforded $\mathbf{4 0}(15.9 \mathrm{mg}, 0.0379 \mathrm{mmol}, 63 \%$ yield $)$ as a colorless oil. $40:$ $\mathrm{R}_{\mathrm{f}}=0.60\left(\mathrm{SiO}_{2}\right.$, hexanes/EtOAc 2:1, v/v); $[\alpha]_{\mathrm{D}}^{23}=+12.3\left(c=0.30\right.$, EtOAc); FT-IR (film): $v_{\max } 2954,2926$, 2873, 1497, 1454, 1370, 1333, 1311, 1259, 1196, 1123, 1096, 1032, 1016, 1004, 968, 910, 897, 845, 738, $699 \mathrm{~cm}^{-1} ;{ }^{1} \mathrm{H}$ NMR $\left(600 \mathrm{MHz}, \mathrm{CDCl}_{3}\right): \delta 7.36-7.31(\mathrm{~m}, 4 \mathrm{H}), 7.29-7.27(\mathrm{~m}, 1 \mathrm{H}), 5.13(\mathrm{dd}, J=5.8,3.7 \mathrm{~Hz}$, $1 \mathrm{H}), 4.65-4.47$ (m, 2 H), $3.94(\mathrm{dd}, J=5.5,2.4 \mathrm{~Hz}, 1 \mathrm{H}), 3.85(\mathrm{dt}, J=3.8,1.9 \mathrm{~Hz}, 1 \mathrm{H}), 3.80(\mathrm{td}, J=7.8,3.7 \mathrm{~Hz}$, $1 \mathrm{H}), 3.68(\mathrm{~d}, J=4.4 \mathrm{~Hz}, 1 \mathrm{H}), 3.54-3.40(\mathrm{~m}, 2 \mathrm{H}), 3.37$ (s, $3 \mathrm{H}), 3.01(\mathrm{~d}, J=2.8 \mathrm{~Hz}, 1 \mathrm{H}), 2.35$ (dq, $J=11.2$, $7.0 \mathrm{~Hz}, 1 \mathrm{H}), 2.26(\mathrm{dd}, J=14.4,5.8 \mathrm{~Hz}, 1 \mathrm{H}), 2.19-2.09(\mathrm{~m}, 2 \mathrm{H}), 2.07-1.98$ (m, 2H), 1.81 (dt, $J=16.1$, $5.0 \mathrm{~Hz}, 1 \mathrm{H}), 1.70(\mathrm{t}, J=12.9 \mathrm{~Hz}, 1 \mathrm{H}), 1.40-1.31(\mathrm{~m}, 2 \mathrm{H}), 1.03(\mathrm{~d}, J=6.6 \mathrm{~Hz}, 3 \mathrm{H}), 0.94(\mathrm{~d}, J=6.9 \mathrm{~Hz}$, 
$3 \mathrm{H}) \mathrm{ppm} ;{ }^{13} \mathrm{C} \mathrm{NMR}\left(151 \mathrm{MHz}, \mathrm{CDCl}_{3}\right.$ ): $\delta 138.6,128.5,128.4,128.0,127.9,127.6,106.6,104.9,85.8,76.7$, 74.0, 73.6, 73.3, 72.3, 63.7, 55.7, 46.7, 41.8, 36.0, 34.8, 30.4, 30.2, 17.9, 17.4 ppm; HRMS (ESI-TOF) $\mathrm{m} / z$ : $[\mathrm{M}+\mathrm{Na}]^{+}$Calcd. for $\mathrm{C}_{24} \mathrm{H}_{34} \mathrm{O}_{6} \mathrm{Na}^{+}$441.2248; Found 441.2251.

3,7:6,10-Dianhydro-12-O-[tert-butyl(diphenyl)silyl]-2,5,8,11-tetradeoxy-8-methyl-L-arabino-Lgalacto-dodecofuranose (42) and 3,7:6,10-dianhydro-2,5,8,11-tetradeoxy-8-methyl-L-arabino-Lgalacto-dodecofuranose (S4): To a stirred solution of ketone $\mathbf{4 1 ^ { 5 }}(1.35 \mathrm{~g}, 2.57 \mathrm{mmol}, 1.0$ equiv) in THF
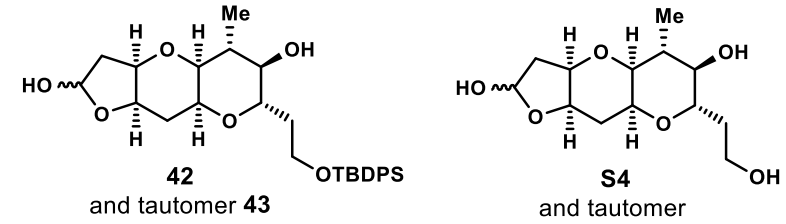
$(20 \mathrm{~mL})$ at $-78^{\circ} \mathrm{C}$ was added dropwise $\mathrm{LiAlH}_{4}(1.0 \mathrm{M}$ in THF, $2.83 \mathrm{~mL}, 2.83 \mathrm{mmol}, 1.1$ equiv). The resulting mixture was stirred for $10 \mathrm{~min}$ and then carefully quenched by dropwise addition of a mixture of $\mathrm{AcOH} / \mathrm{H}_{2} \mathrm{O}\left(\mathrm{AcOH}, 20 \mathrm{~mL} ; \mathrm{H}_{2} \mathrm{O}, 10 \mathrm{~mL}\right)$, and the resulting mixture was warmed to $50^{\circ} \mathrm{C}$ and stirred for $1 \mathrm{~h}$. The resulting mixture was allowed to cool to $23^{\circ} \mathrm{C}$ and concentrated under reduced pressure to give crude 42. Flash column chromatography $\left(\mathrm{SiO}_{2}\right.$, hexanes/ EtOAc $2: 1, v / v \rightarrow 1: 2, v / v$ ) of the residue afforded $\mathbf{4 2 / 4 3}$ (mixture of tautomers, $948 \mathrm{mg}, 1.85 \mathrm{mmol}, 72 \%$ yield) and desilyl product $\mathbf{S 4}(148 \mathrm{mg}, 0.540 \mathrm{mmol}, 21 \%$ yield) as colorless oils. $\mathbf{4 2 / 4 3}$ (mixture of tautomers): $\mathrm{R}_{\mathrm{f}}=0.30\left(\mathrm{SiO}_{2}\right.$, hexanes/EtOAc $\left.1: 1, v / v\right) ;[\alpha]_{\mathrm{D}}^{23}=-14.0(c=0.38$, EtOAc, mixture of tautomers $)$; FT-IR (film, mixture of tautomers): $v_{\max } 3412,2929,2891,2858,1724,1471,1428,1361,1111,1086,1028$, $1008,823,739,703 \mathrm{~cm}^{-1} ;{ }^{1} \mathrm{H}$ NMR (600 MHz, $\mathrm{C}_{6} \mathrm{D}_{6}$, mixture of tautomers): $\delta 9.37(\mathrm{~s}, 0.18 \mathrm{H}), 7.95-7.64$ (m, 4H), 7.36-7.21 (m, 6H), $5.46(\mathrm{dd}, J=5.3,3.0 \mathrm{~Hz}, 0.31 \mathrm{H}), 5.42(\mathrm{~d}, J=5.1 \mathrm{~Hz}, 0.41 \mathrm{H}), 4.32-4.24(\mathrm{~m}$, $0.26 \mathrm{H}), 4.01(\mathrm{dt}, J=9.8,4.0 \mathrm{~Hz}, 0.45 \mathrm{H}), 3.97(\mathrm{td}, J=6.1,3.7 \mathrm{~Hz}, 0.32 \mathrm{H}), 3.86(\mathrm{dtd}, J=9.6,6.4,5.8,3.0 \mathrm{~Hz}$, $1.24 \mathrm{H}), 3.83-3.79(\mathrm{~m}, 0.99 \mathrm{H}), 3.68-3.63(\mathrm{~m}, 0.44 \mathrm{H}), 3.61(\mathrm{dq}, J=6.3,3.0 \mathrm{~Hz}, 0.32 \mathrm{H}), 3.50-3.44(\mathrm{~m}$, $0.75 \mathrm{H}), 3.41(\mathrm{t}, J=3.9 \mathrm{~Hz}, 0.58 \mathrm{H}), 3.37(\mathrm{dt}, J=8.5,4.4 \mathrm{~Hz}, 0.36 \mathrm{H}), 3.32$ (brs, 0.28H), 3.27-3.22 (m, $0.49 \mathrm{H}), 3.17(\mathrm{~s}, 0.29 \mathrm{H}), 3.10(\mathrm{t}, J=4.1 \mathrm{~Hz}, 0.65 \mathrm{H}), 3.05(\mathrm{t}, J=6.8 \mathrm{~Hz}, 0.33 \mathrm{H}), 2.88(\mathrm{dd}, J=6.2,4.4 \mathrm{~Hz}$ $0.35 \mathrm{H}), 2.78$ (dd, $J=4.2,2.6 \mathrm{~Hz}, 0.57 \mathrm{H}), 2.73$ (brs, $0.32 \mathrm{H}), 2.54-2.37$ (m, 0.48 H), 2.19 (ddd, $J=14.6,5.6$, $3.4 \mathrm{~Hz}, 0.51 \mathrm{H}), 2.15-2.06(\mathrm{~m}, 0.61 \mathrm{H}), 2.06-1.95(\mathrm{~m}, 1.96 \mathrm{H}), 1.93-1.84(\mathrm{~m}, 0.61 \mathrm{H}), 1.79$ (tdd, $J=10.2$, 8.5, $5.2 \mathrm{~Hz}, 0.83 \mathrm{H}), 1.75-1.58(\mathrm{~m}, 1.14 \mathrm{H}), 1.51(\mathrm{dt}, J=13.7,4.9 \mathrm{~Hz}, 0.48 \mathrm{H}), 1.45$ (dddd, $J=14.2,8.2,7.0$, $3.8 \mathrm{~Hz}, 0.24 \mathrm{H}), 1.38(\mathrm{ddd}, J=14.5,5.9,4.0 \mathrm{~Hz}, 0.52 \mathrm{H}), 1.21-1.13(\mathrm{~m}, 9 \mathrm{H}), 1.03(\mathrm{~d}, J=7.0 \mathrm{~Hz}, 0.95 \mathrm{H})$, 
$0.81(\mathrm{~d}, J=7.5 \mathrm{~Hz}, 1.40 \mathrm{H}), 0.59(\mathrm{~d}, J=7.9 \mathrm{~Hz}, 0.58 \mathrm{H}) \mathrm{ppm} ;{ }^{13} \mathrm{C} \mathrm{NMR}\left(151 \mathrm{MHz}, \mathrm{C}_{6} \mathrm{D}_{6}\right.$, mixture of tautomers): $\delta 199.3,136.2,136.10,136.08,136.07,136.0,134.4,134.23,134.21,134.1,134.0,133.8$, $130.12,130.06,130.02,129.98,99.6,98.1,78.3,77.3,76.9,76.3,76.2,75.82,75.81,75.6,75.4,74.80$, $74.75,73.6,72.5,71.9,66.1,64.3,61.7,61.7,61.6,61.5,61.1,46.3,42.1,41.7,39.6,39.3,38.4,35.0,34.5$, $33.8,32.7,31.3,29.5,27.15,27.12,27.1,19.44,19.41,15.69,15.65,15.6 \mathrm{ppm}$; HRMS (ESI-TOF) $\mathrm{m} / \mathrm{z}$ : $[\mathrm{M}+\mathrm{Na}]^{+}$Calcd. for $\mathrm{C}_{29} \mathrm{H}_{40} \mathrm{O}_{6} \mathrm{SiNa}^{+}$535.2486; Found 535.2491.

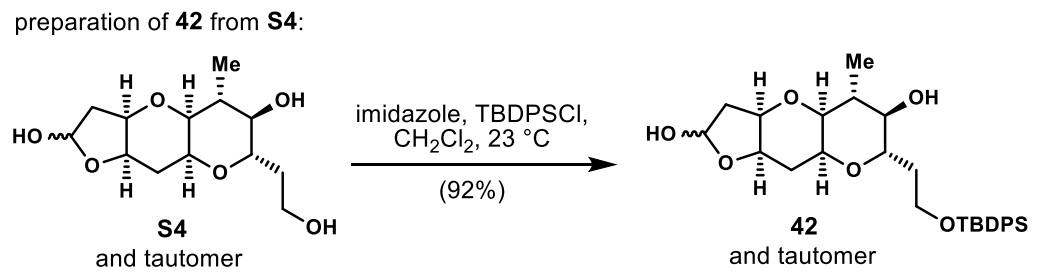

3,7:6,10-Dianhydro-12-O-[tert-butyl(diphenyl)silyl]-2,5,8,11-tetradeoxy-8-methyl-L-arabino-Lgalacto-dodecofuranose (42): To a stirred solution of S4 (mixture of tautomers, $148 \mathrm{mg}, 0.540 \mathrm{mmol}$, 1.0 equiv) in $\mathrm{CH}_{2} \mathrm{Cl}_{2}(5 \mathrm{~mL})$ at $0{ }^{\circ} \mathrm{C}$ were added imidazole (73.5 $\mathrm{mg}, 1.08 \mathrm{mmol}, 2.0$ equiv) and TBDPSCl (178 mg, $0.648 \mathrm{mmol}, 1.2$ equiv). The resulting mixture was allowed to warm to $23^{\circ} \mathrm{C}$ and stirred for $3 \mathrm{~h}$ before it was quenched by the addition of sat. aq. $\mathrm{NaHCO}_{3}$ solution $(10 \mathrm{~mL})$. The layers were separated, and the aqueous layer was extracted with $\mathrm{CH}_{2} \mathrm{Cl}_{2}(3 \times 30 \mathrm{~mL})$. The combined organic layers were washed with brine $(15 \mathrm{~mL})$, dried over $\mathrm{Na}_{2} \mathrm{SO}_{4}$, filtered, and concentrated under reduced pressure. Flash column chromatography $\left(\mathrm{SiO}_{2}\right.$, hexanes/EtOAc 10:1, v/v $\left.\rightarrow 1: 1, v / v\right)$ of the residue afforded 42/43 (mixture of tautomers, $255 \mathrm{mg}, 0.497 \mathrm{mmol}, 92 \%$ yield) as a colorless oil.

\section{4,8:7,11-Dianhydro-13-O-[tert-butyl(diphenyl)silyl]-1,3,6,9,12-pentadeoxy-1-(dimethoxyphosphoryl)-}

9-methyl-L-arabino-L-galacto-tridec-2-ulose (45): To a stirred solution of 42/43 (mixture of tautomers,

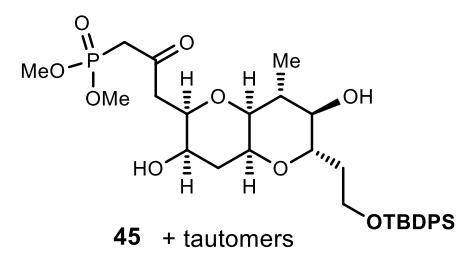
$1.28 \mathrm{~g}, 2.50 \mathrm{mmol}, 1.0$ equiv) and dimethyl diazomethylphosphonate 44 (1.87 g, $12.5 \mathrm{mmol}, 5.0$ equiv) in $\mathrm{CH}_{2} \mathrm{Cl}_{2}(10 \mathrm{~mL})$ at $0{ }^{\circ} \mathrm{C}$ were added $\mathrm{SnCl}_{2}$ $\left(1.18 \mathrm{~g}, 6.24 \mathrm{mmol}, 2.5\right.$ equiv). The resulting mixture was warmed to $23^{\circ} \mathrm{C}$ and stirred for $3 \mathrm{~h}$ before it was diluted with $\mathrm{CH}_{2} \mathrm{Cl}_{2}(30 \mathrm{~mL})$ and quenched 
by the addition of $\mathrm{H}_{2} \mathrm{O}(30 \mathrm{~mL})$. The layers were separated, and the aqueous layer was extracted with $\mathrm{CH}_{2} \mathrm{Cl}_{2}$ $(5 \times 30 \mathrm{~mL})$. The combined organic layers were dried over $\mathrm{Na}_{2} \mathrm{SO}_{4}$, filtered, and concentrated under reduced pressure. Flash column chromatography $\left(\mathrm{SiO}_{2}\right.$, hexanes/EtOAc $2: 1, v / v \rightarrow 1: 2, v / v$; then EtOAc/ MeOH $20: 1, v / v \rightarrow 5: 1, v / v$ ) afforded phosphonate 45 (mixture of tautomers, $1.16 \mathrm{~g}, 1.83 \mathrm{mmol}, 73 \%$ yield) as a colorless oil. 45 (mixture of tautomers): $\mathrm{R}_{\mathrm{f}}=0.70\left(\mathrm{SiO}_{2}, \mathrm{EtOAc} / \mathrm{MeOH} 5: 1, v / v\right) ;[\alpha]_{\mathrm{D}}^{23}=-13.3(c=0.20$, $\mathrm{CHCl}_{3}$ ); FT-IR (film): $v_{\max }$ 3504, 2957, 2930, 2857, 1716, 1472, 1462, 1428, 1391, 1362, 1254, 1187, 1110, 1083, 1030, 823, 741, 704, $688 \mathrm{~cm}^{-1} ;{ }^{1} \mathrm{H} \mathrm{NMR}$ (600 MHz, $\mathrm{CD}_{3} \mathrm{CN}$, major isomer): $\delta 7.72$ (ddt, $J=7.9,3.8$, 1.5 Hz, 4H), 7.52-7.42 (m, 6 H), 4.12-4.06 (m, $1 \mathrm{H}), 3.86-3.80(\mathrm{~m}, 2 \mathrm{H}), 3.77-3.70$ (m, 7 H), 3.69-3.63 (m, 1 H), 3.59 (d, $J=10.7 \mathrm{~Hz}, 1 \mathrm{H}), 3.51-3.43$ (m, $1 \mathrm{H}), 3.40-3.37$ (m, $1 \mathrm{H}), 3.29-3.12$ (m, $3 \mathrm{H}), 3.02$ (dd, $J=17.0$, $8.0 \mathrm{~Hz}, 1 \mathrm{H}), 2.76$ (dd, $J=17.1,4.6 \mathrm{~Hz}, 1 \mathrm{H}), 2.03-1.98$ (m, $1 \mathrm{H}), 1.92(\mathrm{dddd}, J=14.7,10.0,5.5,4.2 \mathrm{~Hz}, 1 \mathrm{H})$, $1.83(\mathrm{dt}, J=14.9,3.2 \mathrm{~Hz}, 1 \mathrm{H}), 1.77(\mathrm{dddd}, J=14.2,8.7,6.9,3.4 \mathrm{~Hz}, 1 \mathrm{H}), 1.12-1.02(\mathrm{~m}, 12 \mathrm{H}) \mathrm{ppm} ;{ }^{13} \mathrm{C}$ NMR (151 MHz, $\mathrm{CD}_{3} \mathrm{CN}$, major isomer): $\delta 201.1$ (d, $\left.J=6.1 \mathrm{~Hz}\right), 136.0,136.0,134.3,134.2,130.39,130.36$, $128.38,128.35,78.8,76.9,72.2,66.5,62.4,61.3,53.12$ (t, $J=5.8 \mathrm{~Hz}, 2 \mathrm{C}), 46.74$ (d, J=1.9 Hz), 42.1, 41.2, 39.3, 34.6, 33.1, 19.3, 15.7 ppm; HRMS (ESI-TOF) $m / z$ : $[\mathrm{M}+\mathrm{Na}]^{+}$Calcd. for $\mathrm{C}_{32} \mathrm{H}_{47} \mathrm{PO}_{9} \mathrm{SiNa}^{+} 657.2619$; Found 657.2620 .

Methyl (14E)-3,7:18,22:21,25-trianhydro-27-O-[tert-butyl(diphenyl)silyl]-2,5,8,9,11,12,14,15,17,20,23,26dodecadeoxy-13-O-(4-methoxybenzyl)-8,12,23-trimethyl-6-O-(triethylsilyl)-L-erythro-D-gulo-Lgalacto-a-D-gluco-heptacos-14-enofuranoside-10,16-diulose (46a): To a stirred solution of phosphonate

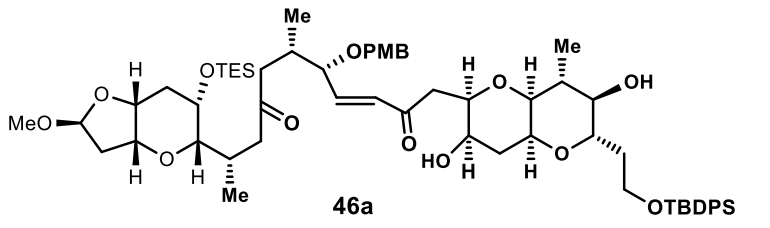
45 (57 mg, $0.090 \mathrm{mmol}, 1.0$ equiv) and 32a $(52 \mathrm{mg}$, $0.090 \mathrm{mmol}, 1.0$ equiv) in THF $(2 \mathrm{~mL})$ at $23^{\circ} \mathrm{C}$ were ( $75 \mu \mathrm{L}, 0.54 \mathrm{mmol}, 6.0$ equiv). The resulting mixture was stirred for $5 \mathrm{~h}$ before it was quenched by the addition of sat. aq. $\mathrm{NH}_{4} \mathrm{Cl}$ solution $(5 \mathrm{~mL})$. The layers were separated, and the aqueous layer was extracted with EtOAc $(3 \times 15 \mathrm{~mL})$. The combined organic layers were washed with brine $(10 \mathrm{~mL})$, dried over $\mathrm{Na}_{2} \mathrm{SO}_{4}$, filtered, and concentrated under reduced pressure. Flash column chromatography $\left(\mathrm{SiO}_{2}\right.$, hexanes/EtOAc $10: 1, v / v \rightarrow 1: 1, v / v)$ of the residue afforded enone $46 \mathrm{a}(54 \mathrm{mg}, 0.0050 \mathrm{mmol}, 55 \%$ yield) as a colorless oil. 
46a: $\mathrm{R}_{\mathrm{f}}=0.70\left(\mathrm{SiO}_{2}\right.$, hexanes/EtOAc $\left.1: 1, v / v\right) ;[\alpha]_{\mathrm{D}}^{23}=-10.6\left(c=0.45, \mathrm{CHCl}_{3}\right)$; FT-IR (film): $v_{\max } 3511$, $2955,2877,1708,1461,1428,1248,1182,1098,1029,823,741 \mathrm{~cm}^{-1} ;{ }^{1} \mathrm{H}$ NMR $\left(600 \mathrm{MHz}, \mathrm{C}_{6} \mathrm{D}_{6}\right): \delta 7.98-$ $7.68(\mathrm{~m}, 4 \mathrm{H}), 7.36-7.29(\mathrm{~m}, 2 \mathrm{H}), 7.29-7.19(\mathrm{~m}, 6 \mathrm{H}), 6.83(\mathrm{~d}, J=8.7 \mathrm{~Hz}, 2 \mathrm{H}), 6.69(\mathrm{dd}, J=16.1,6.4 \mathrm{~Hz}$, $1 \mathrm{H}), 6.34(\mathrm{dd}, J=16.1,1.2 \mathrm{~Hz}, 1 \mathrm{H}), 5.20(\mathrm{dd}, J=5.8,3.9 \mathrm{~Hz}, 1 \mathrm{H}), 4.41(\mathrm{~d}, J=11.4 \mathrm{~Hz}, 1 \mathrm{H}), 4.36(\mathrm{~d}$, $J=10.6 \mathrm{~Hz}, 1 \mathrm{H}), 4.15(\mathrm{~d}, J=11.4 \mathrm{~Hz}, 1 \mathrm{H}), 4.07(\mathrm{~d}, J=10.3 \mathrm{~Hz}, 1 \mathrm{H}), 3.97(\mathrm{~d}, J=10.7 \mathrm{~Hz}, 1 \mathrm{H}), 3.91-3.87$ (m, $1 \mathrm{H}), 3.74(\mathrm{dt}, J=4.6,2.2 \mathrm{~Hz}, 1 \mathrm{H}), 3.70-3.62(\mathrm{~m}, 3 \mathrm{H}), 3.62-3.56(\mathrm{~m}, 3 \mathrm{H}), 3.33(\mathrm{~s}, 3 \mathrm{H}), 3.29(\mathrm{~s}, 3 \mathrm{H})$, 3.23-3.18 (m, $1 \mathrm{H}), 3.14-3.09$ (m, $1 \mathrm{H}), 3.05-2.92(\mathrm{~m}, 2 \mathrm{H}), 2.83$ (brs, $1 \mathrm{H}), 2.73$ (dd, $J=16.1,3.8 \mathrm{~Hz}, 1 \mathrm{H})$, 2.63-2.53 (m, 3 H), 2.51-2.44 (m, $1 \mathrm{H}), 2.31(\mathrm{dd}, J=14.2,5.8 \mathrm{~Hz}, 1 \mathrm{H}), 2.22-2.10(\mathrm{~m}, 4 \mathrm{H}), 2.04$ (ddd, $J=14.2,5.4,3.9 \mathrm{~Hz}, 1 \mathrm{H}), 1.93(\mathrm{dt}, J=14.6,2.9 \mathrm{~Hz}, 1 \mathrm{H}), 1.62(\mathrm{dq}, J=10.1,4.9 \mathrm{~Hz}, 1 \mathrm{H}), 1.49-1.40$ (m, $1 \mathrm{H})$, $1.33(\mathrm{dt}, J=15.3,4.3 \mathrm{~Hz}, 1 \mathrm{H}), 1.17(\mathrm{~s}, 9 \mathrm{H}), 1.10(\mathrm{t}, J=7.9 \mathrm{~Hz}, 9 \mathrm{H}), 0.92$ (d, $J=6.4 \mathrm{~Hz}, 3 \mathrm{H}), 0.90$ (d, $J=6.7 \mathrm{~Hz}, 3 \mathrm{H}), 0.76-0.62(\mathrm{~m}, 6 \mathrm{H}), 0.58(\mathrm{~d}, J=7.9 \mathrm{~Hz}, 3 \mathrm{H}) \mathrm{ppm} ;{ }^{13} \mathrm{C} \mathrm{NMR}\left(151 \mathrm{MHz}, \mathrm{C}_{6} \mathrm{D}_{6}\right): \delta 208.6$, 197.3, 159.8, 145.5, 136.1, 136.0, 134.3, 133.9, 132.6, 130.8, 130.1, 130.0, 129.8, 128.6, 114.2, 105.1, 82.0, 81.9, 78.2, 78.1, 77.6, 77.4, 72.9, 71.9, 71.3, 66.1, 63.5, 61.6, 61.1, 55.2, 54.8, 47.2, 45.7, 42.4, 41.8, 38.4, 34.7, 33.7, 33.4, 32.6, 30.7, 27.1, 19.4, 16.7, 16.5, 15.5, 7.3, 7.3, 5.75, 5.70 ppm; HRMS (ESI-TOF) $m / z$ : $[\mathrm{M}+\mathrm{Na}]^{+}$Calcd. for $\mathrm{C}_{61} \mathrm{H}_{90} \mathrm{O}_{13} \mathrm{Si}_{2} \mathrm{Na}^{+}$1109.5812; Found 1109.5799.

\section{Methyl (14E)-13-O-acetyl-3,7:18,22:21,25-trianhydro-27-O-[tert-butyl(diphenyl)silyl]-} 2,5,8,9,11,12,14,15,17,20,23,26-dodecadeoxy-8,12,23-trimethyl-6-O-(triethylsilyl)-L-erythro-D-gulo-Lgalacto-a-D-gluco-heptacos-14-enofuranoside-10,16-diulose (46b): To a stirred solution of phosphonate

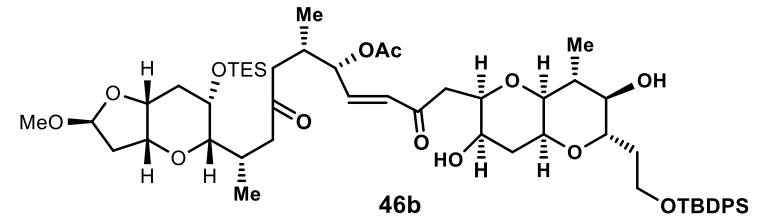
45 (857 mg, $1.35 \mathrm{mmol}, 1.0$ equiv) and 32b (676 mg, $1.35 \mathrm{mmol}, 1.0$ equiv) in $\mathrm{THF}(5 \mathrm{~mL})$ at $23^{\circ} \mathrm{C}$ were added $\mathrm{LiBr}\left(352 \mathrm{mg}, 4.05 \mathrm{mmol}\right.$, 3.0 equiv) and $\mathrm{Et}_{3} \mathrm{~N}$

$(1.13 \mathrm{~mL}, 8.10 \mathrm{mmol}, 6.0$ equiv). The resulting mixture was stirred for $5 \mathrm{~h}$ before it was quenched by the addition of sat. aq. $\mathrm{NH}_{4} \mathrm{Cl}$ solution $(20 \mathrm{~mL})$. The layers were separated, and the aqueous layer was extracted with $\mathrm{CH}_{2} \mathrm{Cl}_{2}(3 \times 50 \mathrm{~mL})$. The combined organic layers were washed with brine $(30 \mathrm{~mL})$, dried over $\mathrm{Na}_{2} \mathrm{SO}_{4}$, filtered, and concentrated under reduced pressure. Flash column chromatography $\left(\mathrm{SiO}_{2}\right.$, hexanes/EtOAc $5: 1, v / v \rightarrow 1: 1, v / v)$ of the residue afforded enone $46 \mathbf{b}(722 \mathrm{mg}, 0.716 \mathrm{mmol}, 53 \%$ yield $)$ as a colorless oil. 18: $\mathrm{R}_{\mathrm{f}}=0.60\left(\mathrm{SiO}_{2}\right.$, hexanes/EtOAc $\left.1: 1, v / v\right) ;[\alpha]_{\mathrm{D}}^{23}=-2.4\left(c=0.50\right.$, EtOAc); FT-IR (film): $v_{\max } 3508,2954$, 
2877, 1744, 1709, 1674, 1457, 1428, 1371, 1233, 1139, 1098, 1060, 1026, 986, 941, 823, 741, $705 \mathrm{~cm}^{-1}$; ${ }^{1} \mathrm{H}$ NMR (600 MHz, $\left.\mathrm{C}_{6} \mathrm{D}_{6}\right)$ : $\delta$ 7.87-7.68 (m, 4H), 7.34-7.30 (m, 2H), 7.30-7.21 (m, 4 H), 6.67 (dd, J=16.0, $5.2 \mathrm{~Hz}, 1 \mathrm{H}), 6.29(\mathrm{dd}, J=16.0,1.6 \mathrm{~Hz}, 1 \mathrm{H}), 5.37(\mathrm{td}, J=5.2,1.6 \mathrm{~Hz}, 1 \mathrm{H}), 5.21(\mathrm{dd}, J=5.8,3.9 \mathrm{~Hz}, 1 \mathrm{H})$, $4.33(\mathrm{dt}, J=10.4,2.9 \mathrm{~Hz}, 1 \mathrm{H}), 3.99(\mathrm{~d}, J=10.2 \mathrm{~Hz}, 1 \mathrm{H}), 3.92(\mathrm{~d}, J=10.7 \mathrm{~Hz}, 1 \mathrm{H}), 3.80(\mathrm{td}, J=6.3,1.1 \mathrm{~Hz}$, $1 \mathrm{H}), 3.75$ (dt, $J=4.7,2.3 \mathrm{~Hz}, 1 \mathrm{H}), 3.69-3.60(\mathrm{~m}, 4 \mathrm{H}), 3.54-3.50(\mathrm{~m}, 1 \mathrm{H}), 3.29(\mathrm{~s}, 3 \mathrm{H}), 3.19$ (dt, $J=10.2$, $2.6 \mathrm{~Hz}, 1 \mathrm{H}), 3.11(\mathrm{~s}, 1 \mathrm{H}), 2.91(\mathrm{dd}, J=6.3,1.9 \mathrm{~Hz}, 2 \mathrm{H}), 2.81(\mathrm{~s}, 1 \mathrm{H}), 2.70(\mathrm{dd}, J=15.9,3.4 \mathrm{~Hz}, 1 \mathrm{H}), 2.62-$ $2.54(\mathrm{~m}, 2 \mathrm{H}), 2.53-2.45(\mathrm{~m}, 1 \mathrm{H}), 2.39-2.26(\mathrm{~m}, 2 \mathrm{H}), 2.20-2.00(\mathrm{~m}, 6 \mathrm{H}), 1.91(\mathrm{dt}, J=14.7,2.9 \mathrm{~Hz}, 1 \mathrm{H})$, $1.67(\mathrm{~s}, 3 \mathrm{H}), 1.66-1.57(\mathrm{~m}, 1 \mathrm{H}), 1.50-1.41(\mathrm{~m}, 1 \mathrm{H}), 1.33$ (dt, J=15.4, 4.4 Hz, $1 \mathrm{H}), 1.17$ (s, 9H), 1.10 (t, $J=8.0 \mathrm{~Hz}, 9 \mathrm{H}), 0.91(\mathrm{~d}, J=6.1 \mathrm{~Hz}, 3 \mathrm{H}), 0.85(\mathrm{~d}, J=6.7 \mathrm{~Hz}, 3 \mathrm{H}), 0.74-0.63(\mathrm{~m}, 6 \mathrm{H}), 0.59(\mathrm{~d}, J=8.0 \mathrm{~Hz}$, 2H)ppm; ${ }^{13} \mathrm{C}$ NMR (151 MHz, $\left.\mathrm{C}_{6} \mathrm{D}_{6}\right): \delta 207.7,197.1,169.3,142.4,136.1,136.0,134.3,133.9,131.5,130.1$, $130.0,128.6,105.1,81.9,78.2,77.9,77.7,77.3,76.0,72.9,71.9,66.0,63.5,61.6,61.1,55.2,47.3,45.1$, $42.7,41.8,38.4,34.7,33.3,32.8,32.6,30.7,27.1,20.4,19.4,16.5,16.3,15.5,7.25,7.22,5.69,5.66$ ppm; HRMS (ESI-TOF) $m / z$ : [M+Na $]^{+}$Calcd. for $\mathrm{C}_{55} \mathrm{H}_{84} \mathrm{O}_{13} \mathrm{Si}_{2} \mathrm{Na}^{+}$1031.5343; Found 1031.5330.

$\left(2 S, 2^{\prime \prime} S, 3 \mathrm{a} R, 3 \mathrm{a}^{\prime} S, 3 \mathrm{a}^{\prime \prime} R, 4 \mathrm{a} S, 4 \mathrm{a}^{\prime \prime} S, 5 R, 5^{\prime} R, 5^{\prime \prime} S, 6 R, 7 S, 7 ' S, 7 \mathrm{a}^{\prime} S, 8 \mathrm{a} S, 8 \mathrm{a}^{\prime \prime} S, 9 \mathrm{a} R, 9 \mathrm{a}^{\prime \prime} R\right)-7-(2-\mathrm{Hydroxy}-$ ethyl)-2"-methoxy-5,5",7'-trimethylicosahydro-2" $H, 3 H, 3 ' H$-dispiro[furo[3,2-b]pyrano[2,3-e]pyran2,2'-furo[3,2-b]pyran-5',7"-furo[3,2-b]pyrano[2,3-e]pyran]-6-ol (47) and (2R,2" $S, 3 a R, 3 a^{\prime} S, 3 a^{\prime \prime} R, 4 a S$, $\left.4 a^{\prime \prime} S, 5 R, 5^{\prime} R, 5^{\prime \prime} S, 6 R, 7 S, 7 ' S, 7 a^{\prime} S, 8 \mathrm{a} S, 8 \mathrm{a}^{\prime \prime} S, 9 \mathrm{a} R, 9 \mathrm{a}^{\prime \prime} R\right)-7-(2-h y d r o x y e t h y l)-2 "$-methoxy-5,5",7'trimethylicosahydro-2" $H, 3 H, 3^{\prime} H$-dispiro[furo[3,2-b]pyrano[2,3-e]pyran-2,2'-furo[3,2-b]pyran5',7"-furo[3,2-b]pyrano[2,3-e]pyran]-6-ol (C16-epi-47): procedure 1, from 46a: To a stirred solution
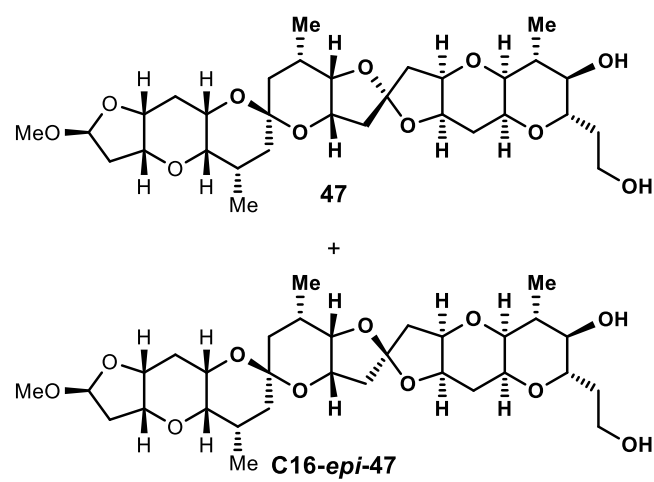
of enone $46 \mathbf{a}(30 \mathrm{mg}, 0.028 \mathrm{mmol}, 1.0$ equiv) in THF $(2 \mathrm{~mL})$ at $23^{\circ} \mathrm{C}$ were added a premixed solution of TBAF/HOAc (TBAF, $1.0 \mathrm{M}$ in THF, $0.42 \mathrm{~mL}, 0.17 \mathrm{mmol}, 15.0$ equiv; HOAc, $8.0 \mu \mathrm{L}$, $0.14 \mathrm{mmol}, 5.0$ equiv). The resulting mixture was stirred for $15 \mathrm{~h}$ before it was diluted with hexanes $(1 \mathrm{~mL})$. The mixture was passed through a pad of $\mathrm{SiO}_{2}$ (hexanes/EtOAc 1:1, v/v; then EtOAc/MeOH 20:1, $v / v \rightarrow 5: 1, v / v)$ to afford the 
corresponding crude triol intermediate (about $15 \mathrm{mg}$ ), which was used in the next step without further purification.

To a stirred solution of the above obtained triol intermediate $(15 \mathrm{mg}, 0.021 \mathrm{mmol}, 1.0$ equiv) in $\mathrm{THF} / \mathrm{MeOH}(3 \mathrm{~mL}, 10: 1, v / v)$ at $23^{\circ} \mathrm{C}$ was added 4,5-dichloro-3,6-dioxocyclohexa-1,4-diene-1,2dicarbonitrile ( $48 \mathrm{mg}, 0.21 \mathrm{mmol}, 10.0$ equiv). The resulting mixture was vigorously stirred for $3 \mathrm{~h}$ before it was quenched by the addition of sat. aq. $\mathrm{NaHCO}_{3}$ solution $(10 \mathrm{~mL})$. The layers were separated, and the aqueous layer was extracted with $\mathrm{CH}_{2} \mathrm{Cl}_{2}(4 \times 10 \mathrm{~mL})$. The combined organic layers were washed with brine $(10 \mathrm{~mL})$, dried over $\mathrm{Na}_{2} \mathrm{SO}_{4}$, filtered, and concentrated under reduced pressure. Flash column chromatography (RP-C18, $\left.\mathrm{MeCN} / \mathrm{H}_{2} \mathrm{O} 1: 2, v / v \rightarrow 1: 1, v / v\right)$ of the residue afforded $47(1.7 \mathrm{mg}, 0.0028 \mathrm{mmol}$, $10 \%$ yield for the two steps) and C16-epi-47 (4.8 mg, $0.0081 \mathrm{mmol}, 29 \%$ yield for the two steps) as colorless oils, respectively. 47: $\mathrm{R}_{\mathrm{f}}=0.60\left(\mathrm{SiO}_{2}\right.$, EtOAc $\left./ \mathrm{MeOH} 10: 1, v / v\right) ;[\alpha]_{\mathrm{D}}^{23}=-31.5(c=0.13$, EtOAc $)$; FT-IR (film): $v_{\max } 3465,2924,2875,1452,1433,1371,1265,1185,1123,1090,1070,1022,1005,972,809 \mathrm{~cm}^{-1}$; ${ }^{1} \mathrm{H}$ NMR $\left(600 \mathrm{MHz}, \mathrm{C}_{6} \mathrm{D}_{6}\right): \delta 5.17(\mathrm{dd}, J=5.9,3.5 \mathrm{~Hz}, 1 \mathrm{H}), 3.90(\mathrm{td}, J=5.8,3.7 \mathrm{~Hz}, 1 \mathrm{H}), 3.83(\mathrm{ddd}, J=9.1$, 6.9, 3.7 Hz, $1 \mathrm{H}), 3.80-3.74(\mathrm{~m}, 2 \mathrm{H}), 3.74-3.63(\mathrm{~m}, 2 \mathrm{H}), 3.59(\mathrm{dt}, J=6.1,2.7 \mathrm{~Hz}, 2 \mathrm{H}), 3.42(\mathrm{t}, J=3.0 \mathrm{~Hz}$, $1 \mathrm{H}), 3.37(\mathrm{dt}, J=5.0,1.4 \mathrm{~Hz}, 1 \mathrm{H}), 3.34(\mathrm{dt}, J=7.3,4.4 \mathrm{~Hz}, 1 \mathrm{H}), 3.20(\mathrm{~s}, 3 \mathrm{H}), 2.98(\mathrm{t}, J=6.8 \mathrm{~Hz}, 1 \mathrm{H}), 2.87$ $(\mathrm{dd}, J=5.7,4.2 \mathrm{~Hz}, 1 \mathrm{H}), 2.74-2.70(\mathrm{~m}, 1 \mathrm{H}), 2.54(\mathrm{~d}, J=14.0 \mathrm{~Hz}, 1 \mathrm{H}), 2.43$ (ddt, $J=12.7,7.3,3.8 \mathrm{~Hz}, 1 \mathrm{H})$, 2.34-2.26 (m, 4 H), 2.12-2.02 (m, 2 H), 1.99-1.91 (m, 2 H), 1.89 (dd, $J=14.1,4.6 \mathrm{~Hz}, 1 \mathrm{H}), 1.79-1.72$ (m, 1H), 1.71-1.64 (m, 3 H), 1.64-1.51 (m, 4H), 1.08 (d, J=6.9 Hz, 3 H), 1.00 (d, J=4.3 Hz, 3H), 0.99 (d, $J=4.4 \mathrm{~Hz}, 3 \mathrm{H}) \mathrm{ppm} ;{ }^{13} \mathrm{C} \mathrm{NMR}\left(151 \mathrm{MHz}, \mathrm{C}_{6} \mathrm{D}_{6}\right): \delta 113.4,104.9,97.2,79.9,77.3,76.9,76.1,75.8,75.4$, 74.1, 74.0, 72.4, 71.7, 64.4, 63.9, 60.0, 55.3, 46.4, 44.5, 42.1, 40.1, 37.6, 37.2, 35.2, 30.4, 29.8, 29.1, 26.3, 18.0, 17.5, 15.8 ppm; HRMS (ESI-TOF) $\mathrm{m} / z$ : $[\mathrm{M}+\mathrm{Na}]^{+}$Calcd. for $\mathrm{C}_{31} \mathrm{H}_{48} \mathrm{O}_{11} \mathrm{Na}^{+}$619.3089; Found 619.3090. C16-epi-47: $\mathrm{R}_{\mathrm{f}}=0.55\left(\mathrm{SiO}_{2}\right.$, EtOAc $\left./ \mathrm{MeOH} 10: 1, v / v\right) ;[\alpha]_{\mathrm{D}}^{23}=-55.0\left(c=0.10\right.$, EtOAc); FT-IR (film): $v_{\max }$ 3454, 2951, 2923, 2874, 1431, 1371, 1322, 1264, 1209, 1196, 1118, 1096, 1049, 1025, 1006, 972, 821, $786 \mathrm{~cm}^{-1} ;{ }^{1} \mathrm{H}$ NMR $\left(600 \mathrm{MHz}, \mathrm{C}_{6} \mathrm{D}_{6}\right): \delta 5.16(\mathrm{dd}, J=5.9,3.6 \mathrm{~Hz}, 1 \mathrm{H}), 4.09$ (ddd, $\left.J=9.3,6.7,3.7 \mathrm{~Hz}, 1 \mathrm{H}\right)$, $3.94(\mathrm{t}, J=2.5 \mathrm{~Hz}, 1 \mathrm{H}), 3.81-3.73(\mathrm{~m}, 3 \mathrm{H}), 3.70(\mathrm{ddd}, J=10.7,6.3,4.1 \mathrm{~Hz}, 1 \mathrm{H}), 3.61-3.49(\mathrm{~m}, 2 \mathrm{H}), 3.45-$ $3.37(\mathrm{~m}, 2 \mathrm{H}), 3.32(\mathrm{dt}, J=4.9,1.5 \mathrm{~Hz}, 1 \mathrm{H}), 3.20(\mathrm{~s}, 3 \mathrm{H}), 3.10(\mathrm{dd}, J=6.8,5.0 \mathrm{~Hz}, 1 \mathrm{H}), 2.96(\mathrm{t}, J=4.8 \mathrm{~Hz}$, $1 \mathrm{H}), 2.69(\mathrm{~d}, J=2.8 \mathrm{~Hz}, 1 \mathrm{H}), 2.44-2.37(\mathrm{~m}, 2 \mathrm{H}), 2.38-2.26(\mathrm{~m}, 4 \mathrm{H}), 2.20-2.10(\mathrm{~m}, 2 \mathrm{H}), 2.09-2.01(\mathrm{~m}$, 
2H), $1.95(\mathrm{dd}, J=14.2,5.9 \mathrm{~Hz}, 1 \mathrm{H}), 1.81(\mathrm{ddt}, J=14.2,7.8,3.9 \mathrm{~Hz}, 1 \mathrm{H}), 1.75-1.67(\mathrm{~m}, 1 \mathrm{H}), 1.65-1.55$ (m, 2H), $1.52(\mathrm{dt}, J=15.6,5.1 \mathrm{~Hz}, 1 \mathrm{H}), 1.49-1.38(\mathrm{~m}, 2 \mathrm{H}), 1.29(\mathrm{dd}, J=13.0,4.3 \mathrm{~Hz}, 1 \mathrm{H}), 1.10(\mathrm{~d}, J=7.1 \mathrm{~Hz}$, $3 \mathrm{H}), 1.01(\mathrm{~d}, J=6.9 \mathrm{~Hz}, 3 \mathrm{H}), 0.99(\mathrm{~d}, J=7.3 \mathrm{~Hz}, 3 \mathrm{H}) \mathrm{ppm} ;{ }^{13} \mathrm{C} \mathrm{NMR}\left(151 \mathrm{MHz}, \mathrm{C}_{6} \mathrm{D}_{6}\right): \delta 115.0,104.9,97.0$, 80.0, 77.3, 76.6, 76.4, 76.05, 76.02, 74.5, 73.9, 72.3, 72.1, 64.5, 63.8, 60.8, 55.4, 46.5 (2C), 42.1, 40.0, 37.7, 37.1, 35.7, 30.9, 30.2, 29.2, 26.2, 18.0, 17.5, 15.8 ppm; HRMS (ESI-TOF) $m / z:[\mathrm{M}+\mathrm{Na}]^{+}$Calcd. for $\mathrm{C}_{31} \mathrm{H}_{48} \mathrm{O}_{11} \mathrm{Na}^{+}$619.3089; Found 619.3092.

$\left(2 S, 2^{\prime \prime} S, 3 \mathrm{a} R, 3 \mathrm{a}^{\prime} S, 3 \mathrm{a}^{\prime \prime} R, 4 \mathrm{a} S, 4 \mathrm{a}^{\prime \prime} S, 5 R, 5^{\prime} R, 5^{\prime \prime} S, 6 R, 7 S, 7 ' S, 7 \mathrm{a}^{\prime} S, 8 \mathrm{a} S, 8 \mathrm{a}^{\prime \prime} S, 9 \mathrm{a} R, 9 \mathrm{a}^{\prime \prime} R\right)-7-(2-\mathrm{Hydroxy-}$ ethyl)-2"-methoxy-5,5",7'-trimethylicosahydro-2" $H, 3 H, 3 ' H$-dispiro[furo[3,2-b]pyrano[2,3-e]pyran2,2'-furo[3,2-b]pyran-5',7"-furo[3,2-b]pyrano[2,3-e]pyran]-6-ol (47) and (2R,2" $S, 3 a R, 3 a^{\prime} S, 3 a^{\prime \prime} R, 4 a S$, $\left.4 \mathrm{a}^{\prime \prime} S, 5 R, 5^{\prime} R, 5^{\prime \prime} S, 6 R, 7 S, 7^{\prime} S, 7 \mathrm{a}^{\prime} S, 8 \mathrm{a} S, 8 \mathrm{a}^{\prime \prime} S, 9 \mathrm{a} R, 9 \mathrm{a}^{\prime \prime} R\right)$-7-(2-hydroxyethyl)-2"-methoxy-5,5",7'-trimethylicosahydro-2" $\mathrm{H}, 3 \mathrm{H}, 3^{\prime} \mathrm{H}$-dispiro[furo[3,2-b]pyrano[2,3-e]pyran-2,2'-furo[3,2-b]pyran-5',7'furo[3,2-b]pyrano[2,3-e]pyran]-6-ol (C16-epi-47): procedure 2, from 46b: To a stirred solution of enone
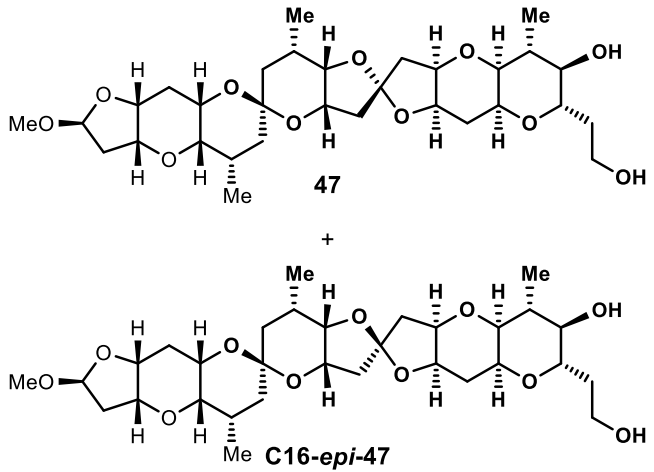
46b $\left(0.10 \mathrm{~g}, 0.099 \mathrm{mmol}, 1.0\right.$ equiv) in THF $(2.5 \mathrm{~mL})$ at $23^{\circ} \mathrm{C}$ were added a premixed solution of TBAF/HOAc (TBAF, $1.0 \mathrm{M}$ in THF, $0.5 \mathrm{~mL}, 0.5 \mathrm{mmol}, 5.1$ equiv; HOAc, $10 \mu \mathrm{L}$, $0.17 \mathrm{mmol}, 1.7$ equiv). The resulting mixture was stirred for $20 \mathrm{~h}$ before $\mathrm{K}_{2} \mathrm{CO}_{3}(0.14 \mathrm{~g}, 0.10 \mathrm{mmol}, 10.0$ equiv) and $\mathrm{MeOH}$ $(15 \mathrm{~mL})$ were added. The mixture was further stirred for $15 \mathrm{~h}$

before it was quenched by the addition of sat. aq. $\mathrm{NH}_{4} \mathrm{Cl}$ solution $(10 \mathrm{~mL})$. The layers were separated, and the aqueous layer was extracted with $\mathrm{CH}_{2} \mathrm{Cl}_{2}(4 \times 20 \mathrm{~mL})$. The combined organic layers were washed with brine $(10 \mathrm{~mL})$, dried over $\mathrm{Na}_{2} \mathrm{SO}_{4}$, filtered, and concentrated under reduced pressure. The residue was passed through a pad of $\mathrm{SiO}_{2}$ (hexanes/EtOAc 1:1, v/v; then EtOAc/MeOH 20:1, v/v $\rightarrow$ 5:1, v/v) to afford the corresponding crude triol intermediate ( $\sim 99 \mathrm{mg}, 0.079 \mathrm{mmol}, 80 \%$ yield), which was divided into three portion used in the next step without further purification.

PPTS-facilitated cyclization: To a stirred solution of the above obtained triol intermediate (16 mg, $0.026 \mathrm{mmol}, 1.0$ equiv) in $\mathrm{CH}_{2} \mathrm{Cl}_{2}(3 \mathrm{~mL})$ at $23^{\circ} \mathrm{C}$ was added PPTS $(6.5 \mathrm{mg}, 0.027 \mathrm{mmol}, 1.0$ equiv). The 
resulting mixture was stirred for $1 \mathrm{~h}$ before it was quenched by the addition of sat. aq. $\mathrm{NaHCO}_{3}$ solution (5 mL). The layers were separated, and the aqueous layer was extracted with $\mathrm{CH}_{2} \mathrm{Cl}_{2}(4 \times 10 \mathrm{~mL})$. The combined organic layers were washed with brine $(10 \mathrm{~mL})$, dried over $\mathrm{Na}_{2} \mathrm{SO}_{4}$, filtered, and concentrated under reduced pressure. Flash column chromatography $\left(\mathrm{RP}-\mathrm{C} 18, \mathrm{MeCN} / \mathrm{H}_{2} \mathrm{O} 1: 2, v / v \rightarrow 1: 1, v / v\right)$ of the residue afforded $47(6.1 \mathrm{mg}, 0.010 \mathrm{mmol}, 33 \%$ yield for the two steps) and C16-epi-47 (5.6 mg, $0.0093 \mathrm{mmol}$, $30 \%$ yield for the two steps) as colorless oils.

( \pm )-CSA-facilitated cyclization: To a stirred solution of the above obtained triol intermediate (16 mg, $0.026 \mathrm{mmol}, 1.0$ equiv) in $\mathrm{CH}_{2} \mathrm{Cl}_{2}(3 \mathrm{~mL})$ at $23{ }^{\circ} \mathrm{C}$ was added ( \pm )-CSA $(6.1 \mathrm{mg}, 0.027 \mathrm{mmol}, 1.0$ equiv). The resulting mixture was stirred for $1 \mathrm{~h}$ before it was quenched by the addition of sat. aq. $\mathrm{NaHCO}_{3}$ solution ( $5 \mathrm{~mL})$. The layers were separated, and the aqueous layer was extracted with $\mathrm{CH}_{2} \mathrm{Cl}_{2}(4 \times 10 \mathrm{~mL})$. The combined organic layers were washed with brine $(10 \mathrm{~mL})$, dried over $\mathrm{Na}_{2} \mathrm{SO}_{4}$, filtered, and concentrated under reduced pressure. Flash column chromatography $\left(\mathrm{RP}-\mathrm{C} 18, \mathrm{MeCN} / \mathrm{H}_{2} \mathrm{O} 1: 2, v / v \rightarrow 1: 1, v / v\right)$ of the residue afforded $47(3.5 \mathrm{mg}, 0.0060 \mathrm{mmol}, 18 \%$ yield for the two steps) and C16-epi-47 (8.7 $\mathrm{mg}, 0.015 \mathrm{mmol}$, $45 \%$ yield for the two steps) as colorless oils.

$\left[\left(2 R, 2^{\prime \prime} S, 3 \mathrm{a} R, 3 \mathrm{a}^{\prime} S, 3 \mathrm{a}^{\prime \prime} R, 4 \mathrm{a} S, 4 \mathrm{a}^{\prime \prime} S, 5 R, 5^{\prime} R, 5^{\prime \prime} S, 6 R, 7 S, 7 ' S, 7 \mathrm{a}^{\prime} S, 8 \mathrm{a} S, 8 \mathrm{a}^{\prime \prime} S, 9 \mathrm{a} R, 9 \mathrm{a}^{\prime \prime} R\right)-6-\mathrm{Hydroxy-2"-}\right.$ methoxy-5,5", 7'-trimethylicosahydro-2" $\mathrm{H,3H}, 3^{\prime} \mathrm{H}$-dispiro[furo[3,2-b]pyrano[2,3-e]pyran-2,2'furo[3,2-b]pyran-5',7"-furo[3,2-b]pyrano[2,3-e]pyran]-7-yl]acetaldehyde (C16-epi-30): To a stirred

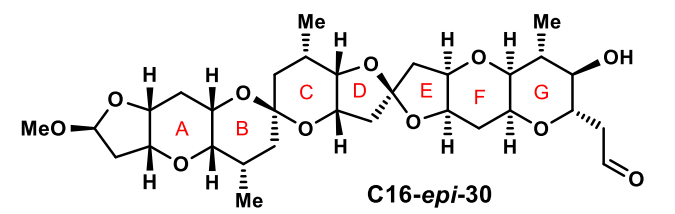
solution of alcohol $47(30 \mathrm{mg}, 0.050 \mathrm{mmol}, 1.0$ equiv $)$ in $\mathrm{CH}_{2} \mathrm{Cl}_{2}(30 \mathrm{~mL})$ at $23{ }^{\circ} \mathrm{C}$ was added $(2,2,6,6$-tetramethylpiperidin-1-yl)oxyl $(3.9 \mathrm{mg}, \quad 0.025 \mathrm{mmol}, \quad 0.5$ equiv $)$ and bis(acetoxy)iodobenzene ( $32 \mathrm{mg}, 0.10 \mathrm{mmol}, 2.0$ equiv). The resulting mixture was stirred for $5 \mathrm{~h}$ before it was quenched by the addition of sat. aq. $\mathrm{Na}_{2} \mathrm{~S}_{2} \mathrm{O}_{3}$ solution $(5 \mathrm{~mL})$. The layers were separated, and the aqueous layer was extracted with $\mathrm{CH}_{2} \mathrm{Cl}_{2}(3 \times 10 \mathrm{~mL})$. The combined organic layers were washed with brine $(10 \mathrm{~mL})$, dried over $\mathrm{Na}_{2} \mathrm{SO}_{4}$, filtered, and concentrated under reduced pressure. Flash column chromatography $\left(\mathrm{SiO}_{2}\right.$, hexanes/EtOAc $\left.1: 1, v / v \rightarrow 1: 2, v / v\right)$ affording aldehyde 30 (23 mg, $0.038 \mathrm{mmol}$, $77 \%$ yield $)$ as a colorless oil. 30: $\mathrm{R}_{\mathrm{f}}=0.50\left(\mathrm{SiO}_{2}, 100 \%\right.$ EtOA $) ;[\alpha]_{\mathrm{D}}^{23}=-36.6(c=0.16$, EtOAc $)$; FT-IR 
(film): $v_{\max } 3468,2926,2874,1724$ 1433, 1370, 1321, 1263, 1196, 1100, 1051, 1025, 1006, 973, $845 \mathrm{~cm}^{-1}$; ${ }^{1} \mathrm{H}$ NMR (600 MHz, $\left.\mathrm{C}_{6} \mathrm{D}_{6}\right): \delta 9.55(\mathrm{dd}, J=3.5,1.5 \mathrm{~Hz}, 1 \mathrm{H}), 5.16(\mathrm{dd}, J=5.9,3.5 \mathrm{~Hz}, 1 \mathrm{H}), 4.30$ (ddd, $J=9.9$, $6.5,4.0 \mathrm{~Hz}, 1 \mathrm{H}), 3.83(\mathrm{t}, J=2.5 \mathrm{~Hz}, 1 \mathrm{H}), 3.77(\mathrm{dd}, J=4.8,2.0 \mathrm{~Hz}, 2 \mathrm{H}), 3.59-3.55(\mathrm{~m}, 2 \mathrm{H}), 3.40(\mathrm{dd}, J=5.8$, $4.0 \mathrm{~Hz}, 1 \mathrm{H}), 3.35(\mathrm{dt}, J=7.1,4.5 \mathrm{~Hz}, 1 \mathrm{H}), 3.32(\mathrm{~d}, J=4.8 \mathrm{~Hz}, 1 \mathrm{H}), 3.20(\mathrm{~s}, 3 \mathrm{H}), 2.92-2.89(\mathrm{~m}, 2 \mathrm{H}), 2.69$ $(\mathrm{d}, J=2.9 \mathrm{~Hz}, 1 \mathrm{H}), 2.44-2.38(\mathrm{~m}, 2 \mathrm{H}), 2.37-2.24(\mathrm{~m}, 6 \mathrm{H}), 2.21-2.10(\mathrm{~m}, 2 \mathrm{H}), 2.11-2.01(\mathrm{~m}, 3 \mathrm{H}), 1.92$ $(\mathrm{dd}, J=14.2,5.9 \mathrm{~Hz}, 1 \mathrm{H}), 1.62-1.49(\mathrm{~m}, 3 \mathrm{H}), 1.50-1.40(\mathrm{~m}, 2 \mathrm{H}), 1.30(\mathrm{dd}, J=12.9,4.3 \mathrm{~Hz}, 1 \mathrm{H}), 1.07$ (d, $J=7.1 \mathrm{~Hz}, 3 \mathrm{H}), 1.01(\mathrm{~d}, J=6.9 \mathrm{~Hz}, 3 \mathrm{H}), 0.89(\mathrm{~d}, J=7.3 \mathrm{~Hz}, 3 \mathrm{H}) \mathrm{ppm} ;{ }^{13} \mathrm{C} \mathrm{NMR}\left(151 \mathrm{MHz}, \mathrm{C}_{6} \mathrm{D}_{6}\right): \delta 200.5$, $114.8,104.9,97.0,79.9,77.4,76.4,76.2,76.1,73.8,73.6,72.5,72.3,72.1,64.8,63.8,55.4,46.7,46.4$, 46.2, 42.1, 39.9, 37.7, 37.1, 30.5, 30.3, 29.2, 26.2, 18.1, 17.5, $15.6 \mathrm{ppm}$; HRMS (ESI-TOF) $m / z:[\mathrm{M}+\mathrm{Na}]^{+}$ Calcd. for $\mathrm{C}_{31} \mathrm{H}_{46} \mathrm{O}_{11} \mathrm{Na}^{+}$617.2932; Found 617.2933.

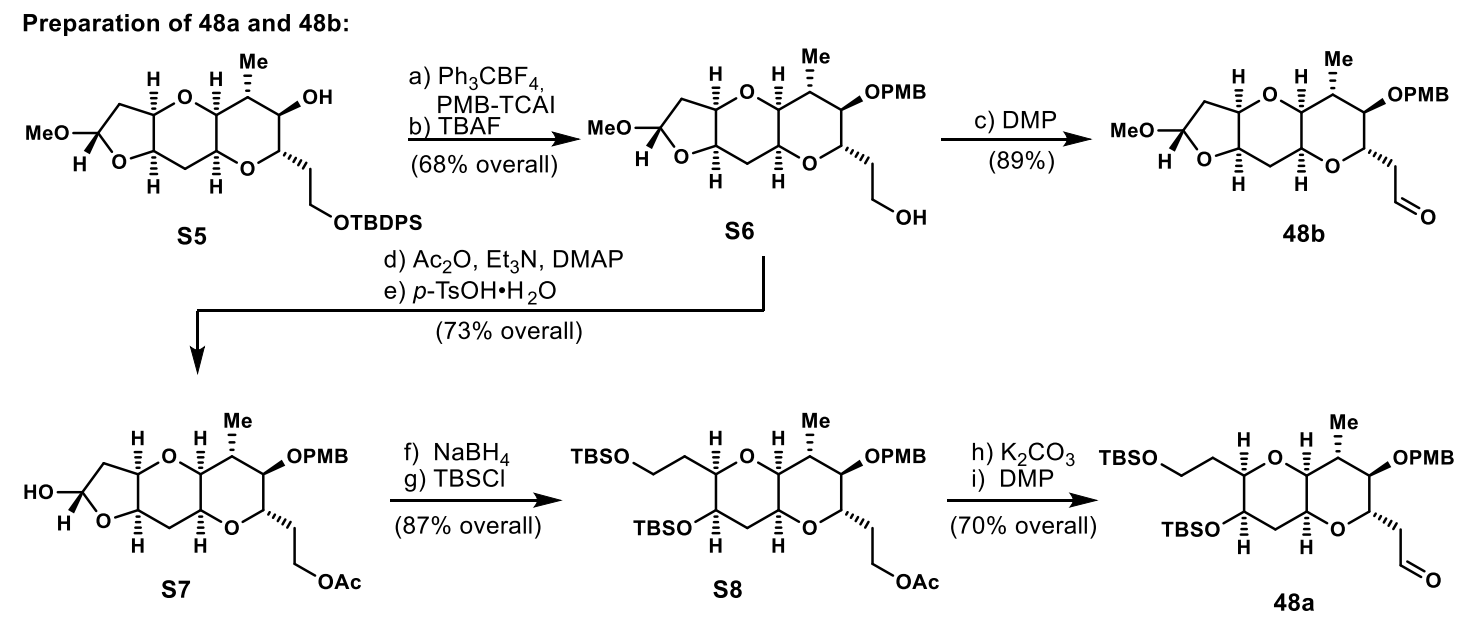

Methyl 3,7:6,10-dianhydro-2,5,8,11-tetradeoxy-9-O-(4-methoxybenzyl)-8-methyl-L-arabino- $\beta$-Lgalacto-dodecofuranoside (S6): To a stirred solution of $\mathbf{S 5}^{5}\left(378 \mathrm{mg}, 0.718 \mathrm{mmol}, 1.0\right.$ equiv) in $\mathrm{Et}_{2} \mathrm{O}$

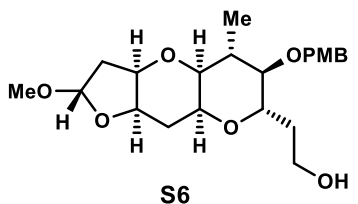
$(60 \mathrm{~mL})$ at $0{ }^{\circ} \mathrm{C}$ was added 4-methoxybenzyl-2,2,2-trichloroacetimidate (PMBTCAI; $305 \mathrm{mg}, 1.08 \mathrm{mmol}, 1.5$ equiv) and triphenylcarbenium tetrafluoroborate ( $47.5 \mathrm{mg}, 0.144 \mathrm{mmol}, 0.2$ equiv). The resulting mixture was warmed to $23^{\circ} \mathrm{C}$ and stirred for $1 \mathrm{~h}$ before it was quenched by the addition of sat. aq. $\mathrm{NaHCO}_{3}$ solution $(20 \mathrm{~mL})$. The layers were separated, and the aqueous layer was extracted with EtOAc $(3 \times 30 \mathrm{~mL})$. The combined organic layers were 
washed with brine $(20 \mathrm{~mL})$, dried over $\mathrm{Na}_{2} \mathrm{SO}_{4}$, filtered, and concentrated under reduced pressure. Flash column chromatography $\left(\mathrm{SiO}_{2}\right.$, hexanes/EtOAc $\left.2: 1, v / v \rightarrow 1: 1, v / v\right)$ of the residue afforded the corresponding PMB-protected alcohol derivative, which was used for the next step without further purification.

To a stirred solution of the above obtained PMB-protected alcohol in THF (30 mL) was added TBAF $\left(1.0 \mathrm{M}\right.$ in THF; $1.08 \mathrm{~mL}, 1.08 \mathrm{mmol}, 1.5$ equiv) at $0^{\circ} \mathrm{C}$. The resulting mixture was allowed to warm to $23^{\circ} \mathrm{C}$ and stirred for $1 \mathrm{~h}$. The resulting mixture was quenched by the addition of sat. aq. $\mathrm{NH}_{4} \mathrm{Cl}$ solution $(20 \mathrm{~mL})$ and extracted with EtOAc $(3 \times 50 \mathrm{~mL})$. The combined organic layers were washed with brine $(30 \mathrm{~mL})$, dried over $\mathrm{Na}_{2} \mathrm{SO}_{4}$, filtered, and concentrated under reduced pressure. Flash column chromatography $\left(\mathrm{SiO}_{2}\right.$, hexanes/EtOAc 1:1, v/v $\rightarrow$ 1:5, v/v) of the residue afforded diol $\mathbf{S 6}(199 \mathrm{mg}, 0.488 \mathrm{mmol}, 68 \%$ yield for the two steps) as a white foam. S6: $\mathrm{R}_{\mathrm{f}}=0.60\left(\mathrm{SiO}_{2}, 100 \%\right.$ EtOAc); $[\alpha]_{\mathrm{D}}^{23}=-8.5\left(c=0.50, \mathrm{CHCl}_{3}\right)$; FT-IR (film): $v_{\max } 3486,2908,2837,1612,1513,1463,1351,1302,1248,1175,1098,1037,990 \mathrm{~cm}^{-1} ;{ }^{1} \mathrm{H}$ NMR $\left(600 \mathrm{MHz}, \mathrm{CDCl}_{3}\right): \delta 7.24(\mathrm{~d}, J=8.6 \mathrm{~Hz}, 2 \mathrm{H}), 6.86(\mathrm{~d}, J=8.6 \mathrm{~Hz}, 2 \mathrm{H}), 5.15(\mathrm{dd}, J=5.7,3.2 \mathrm{~Hz}, 1 \mathrm{H}), 4.58-$ 4.42 (m, $2 \mathrm{H}), 4.04$ (ddd, $J=10.4,9.2,2.7 \mathrm{~Hz}, 1 \mathrm{H}), 4.01-3.96(\mathrm{~m}, 2 \mathrm{H}), 3.83$ (ddd, $J=11.6,8.0,3.7 \mathrm{~Hz}, 1 \mathrm{H})$, 3.81-3.78 (m, 4H), 3.70 (ddd, $J=10.9,6.1,4.4 \mathrm{~Hz}, 1 \mathrm{H}), 3.37-3.34(\mathrm{~m}, 4 \mathrm{H}), 2.94(\mathrm{dd}, J=9.2,6.0 \mathrm{~Hz}, 1 \mathrm{H})$, $2.25(\mathrm{ddd}, J=14.2,5.8,1.7 \mathrm{~Hz}, 1 \mathrm{H}), 2.23-2.17(\mathrm{~m}, 1 \mathrm{H}), 2.08-1.98(\mathrm{~m}, 3 \mathrm{H}), 1.92(\mathrm{dt}, J=14.5,4.8 \mathrm{~Hz}, 1 \mathrm{H})$, 1.49 (dddd, $J=14.1,10.1,6.1,3.7 \mathrm{~Hz}, 1 \mathrm{H}), 1.10(\mathrm{~d}, J=7.3 \mathrm{~Hz}, 3 \mathrm{H}) \mathrm{ppm} ;{ }^{13} \mathrm{C}$ NMR $\left(151 \mathrm{MHz}, \mathrm{CDCl}_{3}\right): \delta$ $159.4,130.5,129.7,114.0,105.0,82.4,77.1,75.6,73.7,72.3,72.1,64.3,60.8,55.7,55.5,41.1,39.2,34.9$, 30.1, 17.7ppm; HRMS (ESI-TOF) m/z: [M+Na] ${ }^{+}$Calcd. for $\mathrm{C}_{22} \mathrm{H}_{32} \mathrm{O}_{7} \mathrm{Na}^{+} 431.2040$; Found 431.2046.

Methyl 3,7:6,10-dianhydro-2,5,8,11-tetradeoxy-9-O-(4-methoxybenzyl)-8-methyl-L-arabino- $\beta$-Lgalacto-dodecodialdo-1,4-furanoside (48b): To a stirred solution of alcohol S6 (77.6 mg, $0.190 \mathrm{mmol}$,

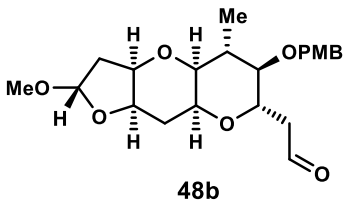
1.0 equiv) in $\mathrm{CH}_{2} \mathrm{Cl}_{2}(10 \mathrm{~mL})$ at $0{ }^{\circ} \mathrm{C}$ was added Dess-Martin periodinane $(121 \mathrm{mg}$, $0.285 \mathrm{mmol}, 1.5$ equiv). The resulting mixture was warmed to $23^{\circ} \mathrm{C}$ and stirred for $1 \mathrm{~h}$ before it was quenched by the addition of sat. aq. $\mathrm{NaHCO}_{3}$ solution $(10 \mathrm{~mL})$ and sat. aq. $\mathrm{Na}_{2} \mathrm{~S}_{2} \mathrm{O}_{3}$ solution $(10 \mathrm{~mL})$. The layers were separated, and the aqueous layer was extracted with $\mathrm{CH}_{2} \mathrm{Cl}_{2}(3 \times 20 \mathrm{~mL})$. The organic layer was washed with brine $(20 \mathrm{~mL})$, dried over $\mathrm{Na}_{2} \mathrm{SO}_{4}$, filtered, and 
concentrated under reduced pressure. Flash column chromatography $\left(\mathrm{SiO}_{2}\right.$, hexanes/EtOAc 10:1, v/v $\rightarrow$ 2:1, $v / v)$ afforded aldehyde $\mathbf{4 8 b}(68.7 \mathrm{mg}, 0.169 \mathrm{mmol}, 89 \%$ yield $)$ as a colorless oil. $\mathbf{4 8 b}: \mathrm{R}_{\mathrm{f}}=0.80\left(\mathrm{SiO}_{2}, 100 \%\right.$ EtOAc); $[\alpha]_{\mathrm{D}}^{23}=+5.6\left(c=0.80, \mathrm{CHCl}_{3}\right) ;$ FT-IR (film): $v_{\max } 2884,1721,1613,1514,1463,1353,1303,1251$, 1222, 1186, 1130, 1101, 1065, 1055, 1037, 987, $821 \mathrm{~cm}^{-1} ;{ }^{1} \mathrm{H}$ NMR (600 MHz, $\left.\mathrm{CDCl}_{3}\right): \delta 9.63(\mathrm{dd}, J=3.3$, $2.0 \mathrm{~Hz}, 1 \mathrm{H}), 7.16(\mathrm{~d}, J=8.6 \mathrm{~Hz}, 2 \mathrm{H}), 6.80(\mathrm{~d}, J=8.6 \mathrm{~Hz}, 2 \mathrm{H}), 5.05(\mathrm{dd}, J=5.4,2.7 \mathrm{~Hz}, 1 \mathrm{H}), 4.41$ (AB quart, $J=10.9 \mathrm{~Hz}, 2 \mathrm{H}), 4.16(\mathrm{td}, J=8.9,4.1 \mathrm{~Hz}, 1 \mathrm{H}), 3.99-3.88(\mathrm{~m}, 2 \mathrm{H}), 3.76-3.73(\mathrm{~m}, 1 \mathrm{H}), 3.73(\mathrm{~s}, 3 \mathrm{H}), 3.32$ $(\mathrm{dd}, J=6.2,4.9 \mathrm{~Hz}, 1 \mathrm{H}), 3.27(\mathrm{~s}, 3 \mathrm{H}), 2.88(\mathrm{dd}, J=9.1,7.6 \mathrm{~Hz}, 1 \mathrm{H}), 2.58(\mathrm{ddd}, J=15.7,4.1,2.0 \mathrm{~Hz}, 1 \mathrm{H})$, 2.34 (ddd, $J=15.7,8.8,3.3 \mathrm{~Hz}, 1 \mathrm{H}), 2.14-2.00$ (m, 3 H), 2.00-1.94 (m, 1 H), 1.93-1.86 (m, $1 \mathrm{H}), 1.09$ (d, $J=7.0 \mathrm{~Hz}, 3 \mathrm{H}) \mathrm{ppm} ;{ }^{13} \mathrm{C} \mathrm{NMR}\left(151 \mathrm{MHz}, \mathrm{CDCl}_{3}\right): \delta 201.6,159.6,130.0,129.8,114.0,104.7,81.4,76.9$, 75.3, 74.4, 72.9, 69.6, 66.1, 55.4, 55.3, 47.0, 40.7, 39.2, 29.0, 16.9 ppm; HRMS (ESI-TOF) $m / z:[\mathrm{M}+\mathrm{Na}]^{+}$ Calcd. for $\mathrm{C}_{22} \mathrm{H}_{30} \mathrm{O}_{7} \mathrm{Na}^{+} 429.1884$; Found 429.1884.

\section{2-O-Acetyl-3,7:6,10-dianhydro-2,5,8,11-tetradeoxy-9-O-(4-methoxybenzyl)-8-methyl-L-arabino- $\beta$ -} L-galacto-dodecofuranose (S7): To a stirred solution of $\mathbf{S 6}$ (130 mg, $0.318 \mathrm{mmol}, 1.0$ equiv) in dry $\mathrm{CH}_{2} \mathrm{Cl}_{2}$

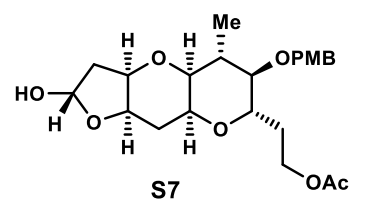

$(10 \mathrm{~mL})$ at $0{ }^{\circ} \mathrm{C}$ were added $\mathrm{Et}_{3} \mathrm{~N}(89.0 \mu \mathrm{L}, 0.636 \mathrm{mmol}, 2.0$ equiv $), \mathrm{Ac}_{2} \mathrm{O}(45.0 \mu \mathrm{L}$, $0.477 \mathrm{mmol}, 1.5$ equiv) and $N, N$-dimethylpyridin-4-amine (DMAP, $19.4 \mathrm{mg}$, $0.159 \mathrm{mmol}, 0.5$ equiv) at $0^{\circ} \mathrm{C}$. The resulting mixture was allowed to warm to $23^{\circ} \mathrm{C}$ and stirred for $5 \mathrm{~h}$ before it was quenched by the addition of $\mathrm{MeOH}(1 \mathrm{~mL})$ and sat. aq. $\mathrm{NaHCO}_{3}$ solution $(10 \mathrm{~mL})$. The layers were separated, and the aqueous layer was extracted with $\mathrm{CH}_{2} \mathrm{Cl}_{2}(3 \times 30 \mathrm{~mL})$. The combined organic layers were washed with brine $(20 \mathrm{~mL})$, dried over $\mathrm{Na}_{2} \mathrm{SO}_{4}$, filtered, and concentrated under reduced pressure. Flash column chromatography $\left(\mathrm{SiO}_{2}\right.$, hexanes/EtOAc 10:1, v/v $\left.\rightarrow 2: 1, v / v\right)$ to afford aldehyde Ac-S6 (129 mg, $0.286 \mathrm{mmol}, 90 \%$ yield) as a colorless oil, which was used without further purification.

To a stirred solution of Ac-S6 (129 mg, $0.286 \mathrm{mmol}, 1.0$ equiv) in acetone $/ \mathrm{H}_{2} \mathrm{O}(6 \mathrm{~mL}, 3: 1, v / v)$ at $23{ }^{\circ} \mathrm{C}$ were added $p-\mathrm{TsOH} \cdot \mathrm{H}_{2} \mathrm{O}\left(54.5 \mathrm{mg}, 0.286 \mathrm{mmol}, 1.0\right.$ equiv). The resulting mixture was warmed to $45^{\circ} \mathrm{C}$ and stirred for $5 \mathrm{~h}$ before it was diluted with sat. aq. $\mathrm{NaHCO}_{3}$ solution $(10 \mathrm{~mL})$. The layers were separated, and the aqueous layer was extracted with $\mathrm{CH}_{2} \mathrm{Cl}_{2}(3 \times 20 \mathrm{~mL})$. The combined organic extracts were dried over 
$\mathrm{Na}_{2} \mathrm{SO}_{4}$, filtered, and concentrated under reduced pressure. Flash column chromatography $\left(\mathrm{SiO}_{2}\right.$, hexanes/EtOAc 1:1, v/v $\rightarrow 1: 5, v / v$ ) of the residue afforded $\mathbf{S} 7$ (mixture of tautomers, $101 \mathrm{mg}, 0.232 \mathrm{mmol}$, $81 \%$ yield). $\mathbf{S} 7$ (mixture of tautomers): $\mathrm{R}_{\mathrm{f}}=0.60\left(\mathrm{SiO}_{2}, 100 \%\right.$ EtOAc $) ;[\alpha]_{\mathrm{D}}^{23}=-33.3\left(c=0.58, \mathrm{CHCl}_{3}\right) ; \mathrm{FT}-$ IR (film): $v_{\max } 3458,2924,1736,1612,1514,1462,1366,1302,1247,1174,1123,1029,822 \mathrm{~cm}^{-1}$; ${ }^{1} \mathrm{H}$ NMR $\left(600 \mathrm{MHz}, \mathrm{CDCl}_{3}\right): \delta 7.18(\mathrm{~d}, J=8.6 \mathrm{~Hz}, 2 \mathrm{H}), 6.79(\mathrm{~d}, J=8.6 \mathrm{~Hz}, 2 \mathrm{H}), 5.31(\mathrm{~d}, J=5.2 \mathrm{~Hz}, 1 \mathrm{H}), 4.50-4.35$ (m, $2 \mathrm{H}), 4.14-4.09$ (m, 2 H), $4.02(\mathrm{ddd}, J=11.1,8.0,6.4 \mathrm{~Hz}, 1 \mathrm{H}), 3.97$ (t, $J=3.1 \mathrm{~Hz}, 1 \mathrm{H}), 3.81$ (dt, $J=4.7$, $2.4 \mathrm{~Hz}, 1 \mathrm{H}), 3.73(\mathrm{~s}, 4 \mathrm{H}), 3.15(\mathrm{t}, J=2.0 \mathrm{~Hz}, 1 \mathrm{H}), 2.92(\mathrm{t}, J=2.0 \mathrm{~Hz}, 1 \mathrm{H}), 2.40(\mathrm{dt}, J=15.6,2.8 \mathrm{~Hz}, 1 \mathrm{H})$, $2.24(\mathrm{qd}, J=8.0,4.5 \mathrm{~Hz}, 1 \mathrm{H}), 2.20-1.99(\mathrm{~m}, 3 \mathrm{H}), 1.98-1.94(\mathrm{~m}, 4 \mathrm{H}), 1.84(\mathrm{dt}, J=15.5,4.5 \mathrm{~Hz}, 1 \mathrm{H}), 1.57$ (dddd, $J=14.3,8.1,7.2,4.0 \mathrm{~Hz}, 1 \mathrm{H}), 1.02(\mathrm{~d}, J=8.0 \mathrm{~Hz}, 3 \mathrm{H}) \mathrm{ppm} ;{ }^{13} \mathrm{C} \mathrm{NMR}\left(151 \mathrm{MHz}, \mathrm{CDCl}_{3}\right): \delta 171.2$, $159.2,123.0,129.6,113.9,99.6,75.6,74.6,74.2,72.6,70.4,62.0,59.4,55.4,42.0,35.6,31.1,28.9,21.2$ 16.6ppm; HRMS (ESI-TOF) m/z: [M+Na] ${ }^{+}$Calcd. for $\mathrm{C}_{23} \mathrm{H}_{32} \mathrm{O}_{8} \mathrm{Na}^{+} 459.1989$; Found 459.1994 .

12-O-Acetyl-3,7:6,10-dianhydro-1,4-bis- $O$-[tert-butyl(dimethyl)silyl]-2,5,8,11-tetradeoxy-9-O-(4methoxybenzyl)-8-methyl-L-arabino-L-galacto-dodecitol (S8): To a stirred solution of S7 (78.1 mg,

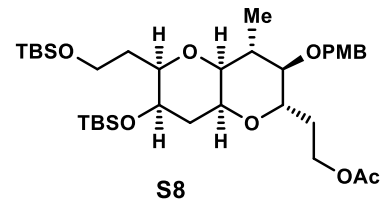
$0.179 \mathrm{mmol}, 1.0$ equiv) in $\mathrm{MeOH}(5 \mathrm{~mL})$ at $0{ }^{\circ} \mathrm{C}$ was added $\mathrm{NaBH}_{4}(13.5 \mathrm{mg}$, $0.358 \mathrm{mmol}, 2.0$ equiv). The resulting mixture was stirred for $0.5 \mathrm{~h}$ before it was quenched by the addition of sat. aq. $\mathrm{NaHCO}_{3}$ solution $(10 \mathrm{~mL})$. The layers were separated, and the aqueous layer was extracted with $\mathrm{CH}_{2} \mathrm{Cl}_{2}(3 \times 20 \mathrm{~mL})$. The combined organic extracts were washed with brine $(20 \mathrm{~mL})$, dried over $\mathrm{Na}_{2} \mathrm{SO}_{4}$, filtered, and concentrated under reduced pressure to afford crude diol without further purification.

To a stirred solution of the above obtained diol in $\mathrm{CH}_{2} \mathrm{Cl}_{2}(5 \mathrm{~mL})$ at $0{ }^{\circ} \mathrm{C}$ were added imidazole $(122 \mathrm{mg}$, $1.79 \mathrm{mmol}, 10.0$ equiv) and $\mathrm{TBSCl}(135 \mathrm{mg}, 0.895 \mathrm{mmol}, 5.0$ equiv). The resulting mixture was allowed to warm to $23{ }^{\circ} \mathrm{C}$ and stirred for $15 \mathrm{~h}$ before it was quenched by the addition of sat. aq. $\mathrm{NaHCO}_{3}$ solution $(20 \mathrm{~mL})$. The layers were separated, and the aqueous layer was extracted with $\mathrm{CH}_{2} \mathrm{Cl}_{2}(3 \times 20 \mathrm{~mL})$. The combined organic layers were washed with brine $(15 \mathrm{~mL})$, dried over $\mathrm{Na}_{2} \mathrm{SO}_{4}$, filtered, and concentrated under reduced pressure. Flash column chromatography $\left(\mathrm{SiO}_{2}\right.$, hexanes/EtOAc 10:1, v/v $\rightarrow$ 5:1, v/v) of the residue afforded $\mathbf{S 8}(104 \mathrm{mg}, 0.156 \mathrm{mmol}, 87 \%$ yield for the two steps $)$ as a colorless oil. S8: $\mathrm{R}_{\mathrm{f}}=0.60\left(\mathrm{SiO}_{2}\right.$, 
hexanes/EtOAc 5:1, v/v); $[\alpha]_{\mathrm{D}}^{23}=-45.0\left(c=0.40, \mathrm{CHCl}_{3}\right)$; FT-IR (film): $v_{\max } 2955,2928,2856,1740,1613$, $1514,1471,1463,1302,1249,1173,1093,1038,835,775 \mathrm{~cm}^{-1} ;{ }^{1} \mathrm{H}$ NMR $\left(600 \mathrm{MHz}, \mathrm{CDCl}_{3}\right): \delta 7.27(\mathrm{~d}$, $J=7.9 \mathrm{~Hz}, 2 \mathrm{H}), 6.87(\mathrm{~d}, J=8.6 \mathrm{~Hz}, 2 \mathrm{H}), 4.56-4.40(\mathrm{~m}, 2 \mathrm{H}), 4.21-4.09(\mathrm{~m}, 2 \mathrm{H}), 3.85(\mathrm{td}, J=8.7,3.6 \mathrm{~Hz}$,

$1 \mathrm{H}), 3.80(\mathrm{~s}, 3 \mathrm{H}), 3.80-3.75(\mathrm{~m}, 2 \mathrm{H}), 3.73(\mathrm{td}, J=5.2,2.8 \mathrm{~Hz}, 1 \mathrm{H}), 3.69$ (ddd, $J=9.8,5.6,4.0 \mathrm{~Hz}, 1 \mathrm{H})$, $3.53(\mathrm{dt}, J=9.8,3.1 \mathrm{~Hz}, 1 \mathrm{H}), 3.38(\mathrm{dd}, J=6.2,4.4 \mathrm{~Hz}, 1 \mathrm{H}), 2.94(\mathrm{dd}, J=9.2,8.0 \mathrm{~Hz}, 1 \mathrm{H}), 2.07-1.98(\mathrm{~m}$, 6H), 1.84-1.69 (m, 3 H), 1.65-1.55 (m, $1 \mathrm{H}), 1.16(\mathrm{~d}, J=6.9 \mathrm{~Hz}, 3 \mathrm{H}), 0.89$ (d, J=4.0Hz, $18 \mathrm{H}), 0.06-0.03$ (m, $12 \mathrm{H}) \mathrm{ppm} ;{ }^{13} \mathrm{C} \mathrm{NMR}\left(151 \mathrm{MHz}, \mathrm{CDCl}_{3}\right): \delta 171.3,159.4,130.5,129.6,113.9,82.0,78.5,73.9,72.9$, $70.4,68.1,66.2,61.6,59.7,55.4,39.4,34.5,34.4,31.7,26.15,26.06,21.2,18.5,18.4,17.2,-4.1,-4.7$, -5.1, -5.2 ppm; HRMS (ESI-TOF) m/z: [M+Na] $]^{+}$Calcd. for $\mathrm{C}_{35} \mathrm{H}_{62} \mathrm{O}_{8} \mathrm{Si}_{2} \mathrm{Na}^{+}$689.3875; Found 689.3877.

3,7:6,10-Dianhydro-9,12-bis-O-[tert-butyl(dimethyl)silyl]-2,5,8,11-tetradeoxy-4-O-(4-methoxybenzyl)-5-methyl-D-lyxo-D-gulo-dodecose (48a): To a stirred solution of S8 (63.1 mg, $0.0944 \mathrm{mmol}$,

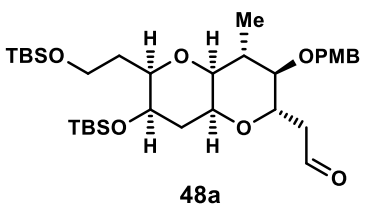

48a 1.0 equiv) in $\mathrm{MeOH}(3 \mathrm{~mL})$ at $0{ }^{\circ} \mathrm{C}$ was added $\mathrm{K}_{2} \mathrm{CO}_{3}(13.0 \mathrm{mg}, 0.0944 \mathrm{mmol}$, 1.0 equiv). The resulting mixture was allowed to warm to $23^{\circ} \mathrm{C}$ and stirred for $2 \mathrm{~h}$. The reaction mixture was quenched by the addition of sat. aq. $\mathrm{NH}_{4} \mathrm{Cl}$ solution $(10 \mathrm{~mL})$. The layers were separated, and the aqueous layer was extracted with EtOAc $(3 \times 15 \mathrm{~mL})$. The organic layer was washed with brine $(10 \mathrm{~mL})$, dried over $\mathrm{Na}_{2} \mathrm{SO}_{4}$, filtered, and concentrated under reduced pressure to give crude primary alcohol $(\sim 60 \mathrm{mg})$, which was used in the next step without further purification.

To a stirred solution of the above obtained crude primary alcohol in $\mathrm{CH}_{2} \mathrm{Cl}_{2}(5 \mathrm{~mL})$ at $0{ }^{\circ} \mathrm{C}$ was added Dess-Martin periodinane $(80.0 \mathrm{mg}, 0.189 \mathrm{mmol}, 2.0$ equiv). The resulting mixture was allowed to warm to $23{ }^{\circ} \mathrm{C}$ and stirred for $1 \mathrm{~h}$ before it was quenched by the addition of sat. aq. $\mathrm{NaHCO}_{3}$ solution $(5 \mathrm{~mL})$ and sat. aq. $\mathrm{Na}_{2} \mathrm{~S}_{2} \mathrm{O}_{3}$ solution $(5 \mathrm{~mL})$, the resulting mixture was further vigorously stirred for $2 \mathrm{~h}$. The layers were separated, and the aqueous layer was extracted with $\mathrm{CH}_{2} \mathrm{Cl}_{2}(3 \times 20 \mathrm{~mL})$. The organic layer was washed with brine $(30 \mathrm{~mL})$, dried over $\mathrm{Na}_{2} \mathrm{SO}_{4}$, filtered, and concentrated under reduced pressure. Flash column chromatography $\left(\mathrm{SiO}_{2}\right.$, hexanes/EtOAc $\left.10: 1, v / v \rightarrow 2: 1, v / v\right)$ afforded 48a $(41.2 \mathrm{mg}, 0.0661 \mathrm{mmol}, 70 \%$ yield for the two steps $)$ as a colorless oil. 48a: $\mathrm{R}_{\mathrm{f}}=0.35\left(\mathrm{SiO}_{2}\right.$, hexanes/EtOAc 5:1, v/v); $[\alpha]_{\mathrm{D}}^{23}=-28.5$ 
$\left(c=0.50, \mathrm{CHCl}_{3}\right)$; FTIR (film): $v_{\max } 2955,2928,2856,1725,1611,1514,1463,1387,1361,1250,1093$ 1037, 835, $775 \mathrm{~cm}^{-1} ;{ }^{1} \mathrm{H}$ NMR $\left(600 \mathrm{MHz}, \mathrm{CDCl}_{3}\right): \delta 9.64(\mathrm{~s}, 1 \mathrm{H}), 7.18(\mathrm{~d}, J=8.2 \mathrm{~Hz}, 2 \mathrm{H}), 6.82(\mathrm{~d}, J=8.1 \mathrm{~Hz}$, 2H), $4.54(\mathrm{dt}, J=9.4,6.2 \mathrm{~Hz}, 1 \mathrm{H}), 4.40(\mathrm{dd}, J=68.9,10.9 \mathrm{~Hz}, 2 \mathrm{H}), 3.81-3.72(\mathrm{~m}, 5 \mathrm{H}), 3.69-3.59(\mathrm{~m}, 2 \mathrm{H})$, $3.44(\mathrm{dd}, J=9.8,2.6 \mathrm{~Hz}, 1 \mathrm{H}), 3.32(\mathrm{t}, J=3.2 \mathrm{~Hz}, 1 \mathrm{H}), 2.96(\mathrm{dd}, J=9.6,5.6 \mathrm{~Hz}, 1 \mathrm{H}), 2.48(\mathrm{dd}, J=6.6,2.9 \mathrm{~Hz}$ 2H), 2.08 (dt, $J=13.6,3.6 \mathrm{~Hz}, 2 \mathrm{H}), 1.81(\mathrm{ddt}, J=14.0,9.1,4.1 \mathrm{~Hz}, 1 \mathrm{H}), 1.69$ (dt, $J=14.5,4.4 \mathrm{~Hz}, 1 \mathrm{H})$, $1.55-1.46(\mathrm{~m}, 1 \mathrm{H}), 1.06(\mathrm{~d}, J=7.3 \mathrm{~Hz}, 3 \mathrm{H}), 0.85$ (brs, $18 \mathrm{H}), 0.03-0.01(\mathrm{~m}, 12 \mathrm{H}) \mathrm{ppm} ;{ }^{13} \mathrm{C}$ NMR (151 MHz, $\left.\mathrm{CDCl}_{3}\right): \delta 201.6,159.5,130.3,129.8,114.0,82.8,79.3,75.2,72.1,68.4,67.3,65.5,59.6,55.4$ 48.3, 39.4, 36.1, 35.2, 26.15, 26.13, 18.6, 18.5, 18.3, -3.9, -4.8, -5.1, -5.2 ppm; HRMS (ESI-TOF) $\mathrm{m} / \mathrm{z}$ : $[\mathrm{M}+\mathrm{Na}]^{+}$Calcd. for $\mathrm{C}_{33} \mathrm{H}_{58} \mathrm{O}_{7} \mathrm{Si}_{2} \mathrm{Na}^{+}$645.3613; Found 645.3610.

\section{NHK reaction toward 50a:}

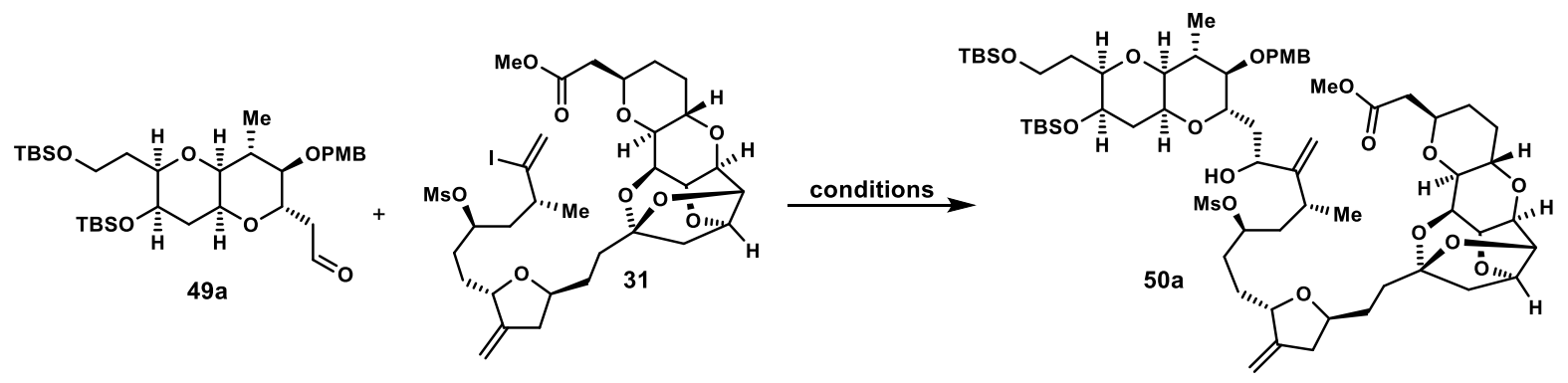

\section{Condition 1:}

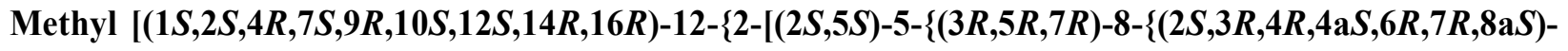
7-\{[tert-butyl(dimethyl)silyl] oxy\}-6-(2-\{[tert-butyl(dimethyl)silyl] $]$ xy\} ethyl)-3-[(4-methoxybenzyl)oxy]-4-methyloctahydropyrano[3,2-b]pyran-2-yl\}-7-hydroxy-5-methyl-6-methylidene-3-[(methylsulfonyl)oxy]octyl\}-4-methylidenetetrahydrofuran-2-yl]ethyl\}-3,8,11,15,17-pentaoxapentacyclo$\left[10.4 .1 .0^{2,7} \cdot 0^{9,16} \cdot 0^{10,14}\right]$ heptadec-4-yl]acetate (50a): In a glove box, to a stirred solution of $48 \mathrm{a}(37.9 \mathrm{mg}$, $0.0608 \mathrm{mmol}, 2.0$ equiv) and $31\left(22.9 \mathrm{mg}, 0.0304 \mathrm{mmol}, 1.0\right.$ equiv) in THF/DMF $(1.0 \mathrm{~mL}, 4: 1, v / v)$ at $23^{\circ} \mathrm{C}$

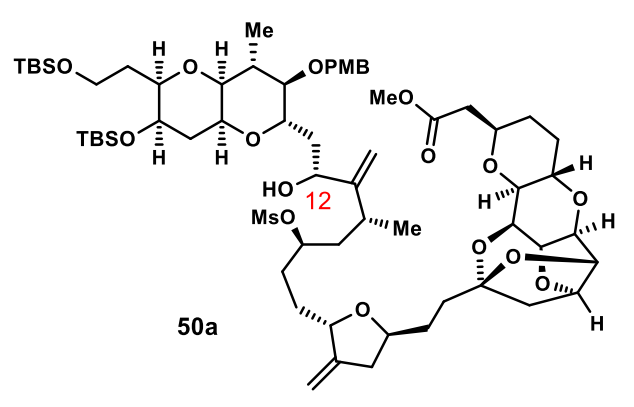

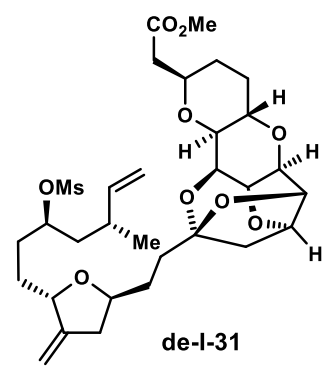

was added $2.0 \% \mathrm{NiCl}_{2} / \mathrm{CrCl}_{2}(37.4 \mathrm{mg}$, 0.3604 mmol, 10.0 equiv). The resulting mixture was vigorously stirred for $24 \mathrm{~h}$ before it was quenched by the addition of $\mathrm{H}_{2} \mathrm{O}(10 \mathrm{~mL})$. The layers 
were separated, and the aqueous layer was extracted with EtOAc $(4 \times 30 \mathrm{~mL})$. The organic layer was washed with brine $(10 \mathrm{~mL})$, dried over $\mathrm{Na}_{2} \mathrm{SO}_{4}$, filtered, and concentrated under reduced pressure. Flash column chromatography $\left(\mathrm{SiO}_{2}\right.$, hexanes/EtOAc hexanes/EtOAc 3:1, v/v $\left.\rightarrow 1: 3, v / v\right)$ of the residue afforded alcohol 50a ( $8.70 \mathrm{mg}, 0.00700 \mathrm{mmol}, 23 \%$ yield, $3: 1 d r$ at $\mathrm{C} 12)$ as a colorless oil, methyl $[(1 S, 2 S, 4 R, 7 S, 9 R, 10 S, 12 S, 14 R, 16 R)$ 12-\{2-[(2S,5S)-4-methylidene-5-\{(3R,5R)-5-methyl-3-[(methylsulfonyl)oxy]hept-6-en-1-yl $\}$ tetrahydrofuran-2-yl]ethyl $\}-3,8,11,15,17$-pentaoxapentacyclo[10.4.1.0 $\left.0^{2,7} \cdot 0^{9,16} \cdot 0^{10,14}\right]$ heptadec-4-yl]acetate (de-I-31; $14.9 \mathrm{mg}, 0.0238 \mathrm{mmol}, 70 \%$ yield) as a colorless oil, and recovered aldehyde $48 \mathrm{a}$ ( $26.5 \mathrm{mg}, 0.0426 \mathrm{mmol}$, $70 \%)$ as a white foam. 50a: $\mathrm{R}_{\mathrm{f}}=0.75\left(\mathrm{SiO}_{2}\right.$, hexanes/EtOAc 1:3, v/v); ${ }^{1} \mathrm{H}$ NMR $\left(600 \mathrm{MHz}, \mathrm{CDCl}_{3}\right): \delta 7.24$ $(\mathrm{d}, J=8.6 \mathrm{~Hz}, 2 \mathrm{H}), 6.86(\mathrm{~d}, J=8.4 \mathrm{~Hz}, 2 \mathrm{H}), 5.14(\mathrm{~s}, 1 \mathrm{H}), 5.00(\mathrm{~d}, J=3.0 \mathrm{~Hz}, 1 \mathrm{H}), 4.87-4.82(\mathrm{~m}, 2 \mathrm{H}), 4.77$ $(\mathrm{td}, J=13.1,12.2,6.8 \mathrm{~Hz}, 1 \mathrm{H}), 4.68(\mathrm{t}, J=4.8 \mathrm{~Hz}, 1 \mathrm{H}), 4.60(\mathrm{t}, J=4.5 \mathrm{~Hz}, 1 \mathrm{H}), 4.58-4.45(\mathrm{~m}, 2 \mathrm{H}), 4.42$ $(\mathrm{dd}, J=3.9,1.9 \mathrm{~Hz}, 1 \mathrm{H}), 4.36(\mathrm{~d}, J=8.2 \mathrm{~Hz}, 1 \mathrm{H}), 4.31-4.24(\mathrm{~m}, 2 \mathrm{H}), 4.20(\mathrm{dd}, J=6.6,4.6 \mathrm{~Hz}, 1 \mathrm{H}), 4.06$ (dd, $J=6.6,4.0 \mathrm{~Hz}, 2 \mathrm{H}), 3.94(\mathrm{dt}, J=10.5,5.2 \mathrm{~Hz}, 1 \mathrm{H}), 3.85-3.75(\mathrm{~m}, 6 \mathrm{H}), 3.74-3.68(\mathrm{~m}, 3 \mathrm{H}), 3.67(\mathrm{~s}$, $3 \mathrm{H}), 3.52(\mathrm{dt}, J=9.8,2.9 \mathrm{~Hz}, 1 \mathrm{H}), 3.37(\mathrm{dd}, J=6.4,4.0 \mathrm{~Hz}, 1 \mathrm{H}), 3.00(\mathrm{~s}, 3 \mathrm{H}), 2.92(\mathrm{dd}, J=9.6,1.9 \mathrm{~Hz}, 1 \mathrm{H})$, 2.72-2.59 (m, 2 H), $2.38(\mathrm{dd}, J=15.8,5.9 \mathrm{~Hz}, 1 \mathrm{H}), 2.36-2.30(\mathrm{~m}, 1 \mathrm{H}), 2.30-2.25(\mathrm{~m}, 1 \mathrm{H}), 2.16-2.08(\mathrm{~m}$, 3H), 2.08-1.99 (m, 3 H), 1.99-1.94 (m, 2 H), 1.93-1.88 (m, 2 H), 1.87-1.80 (m, 3 H), 1.81-1.74 (m, 4H), 1.73-1.64 (m, 5 H), 1.43-1.38 (m, 1 H), 1.17 (d, J=6.7 Hz, 3 H), 1.10-1.06 (m, 3 H), 0.89 (s, $9 \mathrm{H}), 0.89$ (s, 9H), 0.09--0.02 (m, 12 H) ppm; ${ }^{13} \mathrm{C}$ NMR (151 MHz, $\left.\mathrm{CDCl}_{3}\right): \delta 171.7,159.4,156.0,151.4,130.4,129.7$, 129.0, 114.0, 110.5, 105.3, 83.0, 82.2, 82.0, 81.1, 79.1, 78.5, 78.1, 76.7, 75.6, 74.6, 74.5, 74.1, 73.9, 73.9, $73.2,68.4,67.7,66.1,59.7,55.4,51.8,47.2,41.4,40.6,39.1,39.1,38.8,38.8,35.0,34.7,34.6,34.5,32.5$, 31.1, 30.7, 30.3, 30.1, 29.8, 29.7, 26.2, 26.1, 22.1, 18.5, 18.4, 16.9, -3.9, -4.8, -5.1, -5.2 ppm; De-I-31: $\mathrm{R}_{\mathrm{f}}=0.80\left(\mathrm{SiO}_{2}, 100 \%\right.$ EtOAc); $[\alpha]_{\mathrm{D}}^{23}=-68.0$ (c=0.15, EtOAc); FT-IR (film): $v_{\max } 2936,2868,1738,1438$, 1336, 1290, 1263, 1211, 1172, 1133, 1077, 1013, 830, $790 \mathrm{~cm}^{-1} ;{ }^{1} \mathrm{H}$ NMR $\left(600 \mathrm{MHz}, \mathrm{C}_{6} \mathrm{D}_{6}\right): 5.54$ (ddd, $J=17.2,10.3,8.0 \mathrm{~Hz}, 1 \mathrm{H}), 5.15(\mathrm{ddd}, J=17.2,1.8,1.0 \mathrm{~Hz}, 1 \mathrm{H}), 5.00(\mathrm{ddd}, J=10.3,1.8,0.7 \mathrm{~Hz}, 1 \mathrm{H}), 4.91$ $(\mathrm{dtd}, J=9.5,5.8,3.7 \mathrm{~Hz}, 2 \mathrm{H}), 4.80(\mathrm{~d}, J=2.2 \mathrm{~Hz}, 1 \mathrm{H}), 4.55-4.46(\mathrm{~m}, 1 \mathrm{H}), 4.41(\mathrm{dd}, J=4.0,1.9 \mathrm{~Hz}, 1 \mathrm{H})$, 4.37-4.30 (m, $1 \mathrm{H}), 4.14$ (dt, $J=33.3,4.6 \mathrm{~Hz}, 2 \mathrm{H}), 4.01-3.94(\mathrm{~m}, 1 \mathrm{H}), 3.94-3.89$ (m, $1 \mathrm{H}), 3.76$ (dddd, $J=10.8,7.3,5.2,2.1 \mathrm{~Hz}, 1 \mathrm{H}), 3.67(\mathrm{dd}, J=6.6,3.9 \mathrm{~Hz}, 1 \mathrm{H}), 3.32(\mathrm{~s}, 3 \mathrm{H}), 2.62-2.53(\mathrm{~m}, 2 \mathrm{H}), 2.48-2.41$ (m, $1 \mathrm{H}), 2.41-2.36(\mathrm{~m}, 1 \mathrm{H}), 2.33(\mathrm{~s}, 3 \mathrm{H}), 2.18(\mathrm{dd}, J=15.7,5.3 \mathrm{~Hz}, 1 \mathrm{H}), 2.16-2.02(\mathrm{~m}, 3 \mathrm{H}), 2.02-1.97$ 
(m, $1 \mathrm{H}), 1.95-1.91(\mathrm{~m}, 1 \mathrm{H}), 1.78-1.66(\mathrm{~m}, 4 \mathrm{H}), 1.64-1.57(\mathrm{~m}, 2 \mathrm{H}), 1.47$ (dd, $J=13.1,5.1 \mathrm{~Hz}, 1 \mathrm{H}), 1.44-$ $1.39(\mathrm{~m}, 1 \mathrm{H}), 1.35-1.21(\mathrm{~m}, 4 \mathrm{H}), 0.89(\mathrm{~d}, J=6.8 \mathrm{~Hz}, 3 \mathrm{H}) \mathrm{ppm} ;{ }^{13} \mathrm{C} \mathrm{NMR}\left(151 \mathrm{MHz}, \mathrm{C}_{6} \mathrm{D}_{6}\right): \delta 171.1,152.3$, 143.2, 114.7, 110.4, 104.9, 82.4, 81.7, 80.9, 79.0, 78.6, 77.4, 77.0, 74.9, 74.7, 74.3, 68.5, 51.1, 47.4, 41.7, 40.8, 39.1, 38.5, 35.9, 34.5, 31.7, 30.9, 30.8, 30.7, 30.3, 21.0 ppm; HRMS (ESI-TOF) $\mathrm{m} / z$ : $[\mathrm{M}+\mathrm{Na}]^{+}$Calcd. for $\mathrm{C}_{31} \mathrm{H}_{46} \mathrm{O}_{11} \mathrm{SNa}^{+}$649.2653; Found 649.2663

\section{Condition 4:}

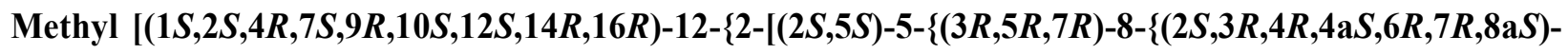
7-\{[tert-butyl(dimethyl)silyl] oxy\}-6-(2-\{[tert-butyl(dimethyl)silyl] $]$ xy $\}$ ethyl)-3-[(4-methoxybenzyl)oxy]-4-methyloctahydropyrano[3,2-b]pyran-2-yl\}-7-hydroxy-5-methyl-6-methylidene-3-[(methylsulfonyl)oxy]octyl\}-4-methylidenetetrahydrofuran-2-yl]ethyl\}-3,8,11,15,17-pentaoxapentacyclo$\left[10.4 .1 .0^{2,7} \cdot 0^{9,16} \cdot 0^{10,14}\right.$ ]heptadec-4-yl]acetate (50a): In a glove box, to a stirred solution of $48 \mathrm{a}(21 \mathrm{mg}$,

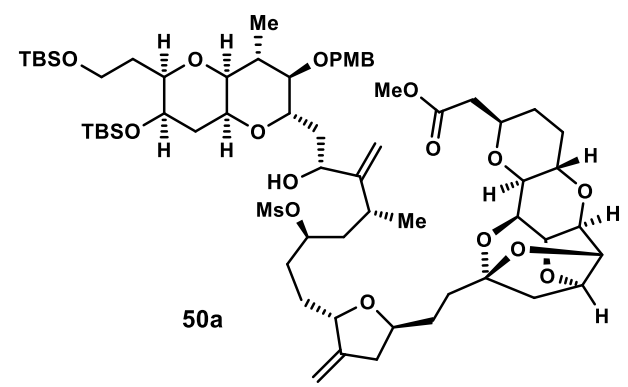
$0.033 \mathrm{mmol}, 1.1$ equiv), 31 (23 mg, $0.030 \mathrm{mmol}, 1.0$ equiv), $\mathrm{Et}_{3} \mathrm{~N}$ $\left(21 \mu \mathrm{L}, 0.15 \mathrm{mmol}, 5.0\right.$ equiv) and $\mathrm{CrCl}_{2}(11 \mathrm{mg}, 0.099 \mathrm{mmol}$, 3.0 equiv) in $\operatorname{THF}(0.5 \mathrm{~mL})$ at $23^{\circ} \mathrm{C}$ was added a premixed solution of ligand 49 (23 mg, $0.075 \mathrm{mmol}, 2.5$ equiv) and $\mathrm{NiCl}_{2}$ $(1.0 \mathrm{mg}, \quad 0.0075 \mathrm{mmol}, \quad 0.25$ equiv) in THF $(0.5 \mathrm{~mL})$. The resulting mixture was stirred for $6 \mathrm{~h}$ before it was quenched by the addition of $\mathrm{H}_{2} \mathrm{O}(10 \mathrm{~mL})$. The layers were separated, and the aqueous layer was extracted with EtOAc $(4 \times 30 \mathrm{~mL})$. The organic layer was washed with brine $(10 \mathrm{~mL})$, dried over $\mathrm{Na}_{2} \mathrm{SO}_{4}$, filtered, and concentrated under reduced pressure. Flash column chromatography $\left(\mathrm{SiO}_{2}\right.$, hexanes/EtOAc $\left.3: 1, v / v \rightarrow 1: 3, v / v\right)$ of the residue afforded 50a $(24 \mathrm{mg}, 0.019 \mathrm{mmol}$, $63 \%$ yield) as a colorless oil. 
Cyclization Toward Methyl [(1S,2S,4R,7S,9R,10S,12S,14R,16R)-12-\{2-[(2S,5S)-5-\{2-[(2R,4R,6S)-6$(\{(2 R, 3 R, 4 R, 4 \mathrm{a} R, 6 S, 7 R, 8 \mathrm{a} R)-7-\{[$ tert-Butyl(dimethyl)silyl]oxy\}-6-(2-\{[tert-butyl(dimethyl)silyl]oxy\}ethyl)-3-[(4-methoxybenzyl)oxy]-4-methyloctahydropyrano[3,2-b]pyran-2-yl\} methyl)-4-methyl-5methylidenetetrahydro-2H-pyran-2-yl] ethyl \}-4-methylidenetetrahydrofuran-2-yl] ethyl\}$3,8,11,15,17-$ pentaoxapentacyclo $\left[10.4 .1 .0^{2,7} \cdot 0^{9,16} \cdot 0^{10,14}\right]$ heptadec-4-yl]acetate (S9):

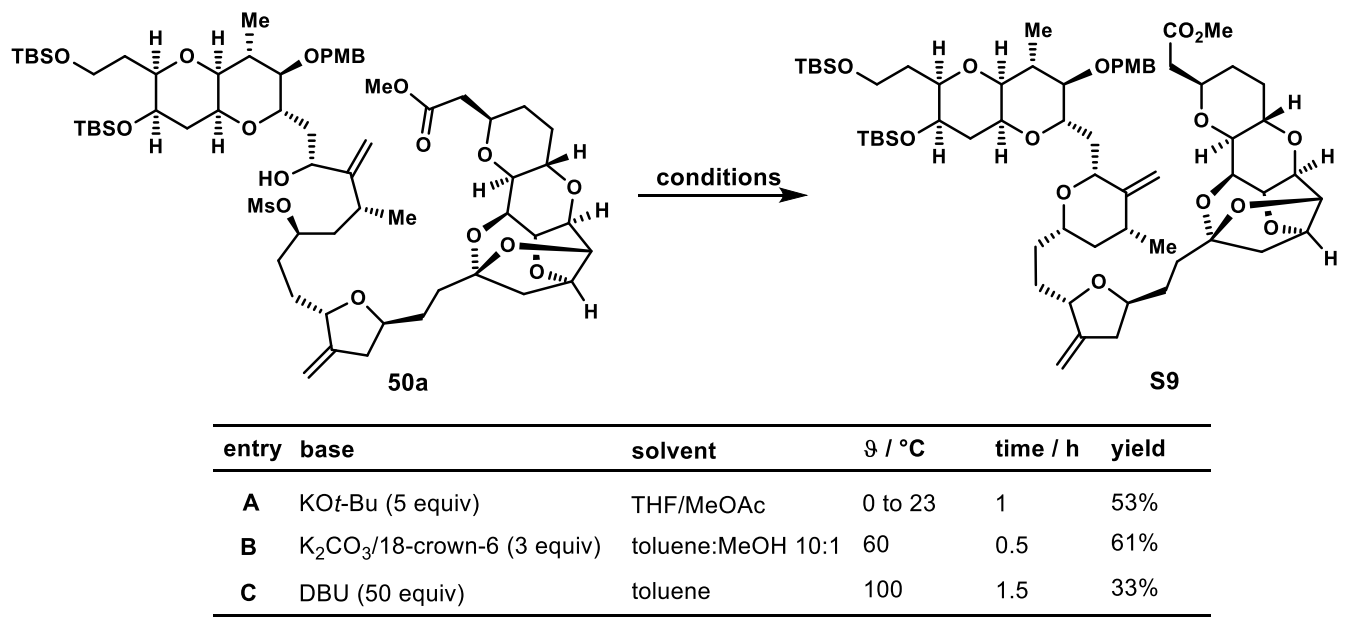

\section{Condition A:}

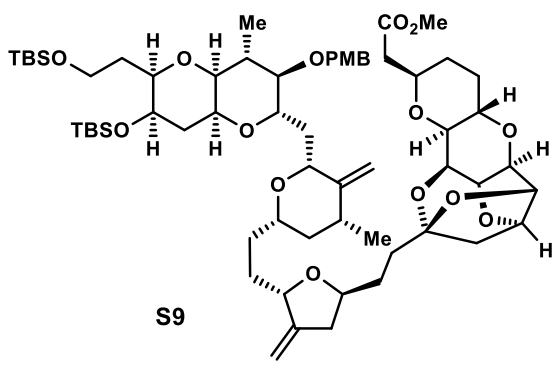

To a stirred solution of 50a $(5.0 \mathrm{mg}, 0.0040 \mathrm{mmol}, 1.0$ equiv) in $\mathrm{THF} / \mathrm{MeOAc}(2 \mathrm{~mL}, 5: 1, v / v)$ at $0^{\circ} \mathrm{C}$ were added $\mathrm{KO} t$-Bu $(4.5 \mathrm{mg}$, $0.040 \mathrm{mmol}, 10.0$ equiv). The resulting mixture was warmed to $23^{\circ} \mathrm{C}$ and stirred for $15 \mathrm{~min}$ before it was quenched by the addition of sat. aq. $\mathrm{NH}_{4} \mathrm{Cl}$ solution $(5 \mathrm{~mL})$. The layers were separated, and the aqueous layer was extracted with EtOAc $(3 \times 10 \mathrm{~mL})$. The combined organic extracts were washed with brine $(10 \mathrm{~mL})$, dried over $\mathrm{Na}_{2} \mathrm{SO}_{4}$, filtered, and concentrated under reduced pressure. Flash column chromatography $\left(\mathrm{SiO}_{2}\right.$, hexanes/EtOAc $\left.3: 1, v / v \rightarrow 1: 1, v / v\right)$ of the residue afforded $\mathbf{S 9}(2.4 \mathrm{mg}, 0.0021 \mathrm{mmol}$, $53 \%$ yield $)$ as a colorless oil. S9: $\mathrm{R}_{\mathrm{f}}=0.70\left(\mathrm{SiO}_{2}\right.$, hexanes/EtOAc 1:1, v/v); $[\alpha]_{\mathrm{D}}^{23}=-33.4\left(c=0.53, \mathrm{CHCl}_{3}\right)$; FT-IR (film): $v_{\max } 2954,2927,2855,1741,1613,1514,1462,1437,1361,1250,1189,1134,1079,1039$, 1008, 902, 834, $775 \mathrm{~cm}^{-1} ;{ }^{1} \mathrm{H}$ NMR $\left(600 \mathrm{MHz} \mathrm{CDCl}_{3}\right): \delta 7.27(\mathrm{~s}, 1 \mathrm{H}), 7.25(\mathrm{~s}, 1 \mathrm{H}), 6.85(\mathrm{~d}, J=8.7 \mathrm{~Hz}$, 2H), $4.96(\mathrm{~d}, J=2.1 \mathrm{~Hz}, 1 \mathrm{H}), 4.93(\mathrm{~s}, 1 \mathrm{H}), 4.83(\mathrm{~d}, J=2.1 \mathrm{~Hz}, 1 \mathrm{H}), 4.79(\mathrm{~d}, J=1.8 \mathrm{~Hz}, 1 \mathrm{H}), 4.68(\mathrm{t}$, $J=4.7 \mathrm{~Hz}, 1 \mathrm{H}), 4.63-4.56(\mathrm{~m}, 2 \mathrm{H}), 4.50(\mathrm{~d}, J=10.5 \mathrm{~Hz}, 1 \mathrm{H}), 4.42(\mathrm{dd}, J=4.0,1.9 \mathrm{~Hz}, 1 \mathrm{H}), 4.38(\mathrm{~s}, 1 \mathrm{H})$, 
$4.30-4.26(\mathrm{~m}, 1 \mathrm{H}), 4.19(\mathrm{dd}, J=6.6,4.6 \mathrm{~Hz}, 1 \mathrm{H}), 4.06(\mathrm{dd}, J=6.7,4.0 \mathrm{~Hz}, 2 \mathrm{H}), 3.95(\mathrm{t}, J=6.5 \mathrm{~Hz}, 1 \mathrm{H})$, 3.86-3.80 (m, $1 \mathrm{H}), 3.79(\mathrm{~s}, 4 \mathrm{H}), 3.78-3.73(\mathrm{~m}, 3 \mathrm{H}), 3.69$ (dd, $J=5.6,4.3 \mathrm{~Hz}, 1 \mathrm{H}), 3.66(\mathrm{~s}, 3 \mathrm{H}), 3.55$ (dt, $J=9.8,3.4 \mathrm{~Hz}, 2 \mathrm{H}), 3.36(\mathrm{dd}, J=7.5,4.8 \mathrm{~Hz}, 1 \mathrm{H}), 3.09$ (t, $J=9.2 \mathrm{~Hz}, 1 \mathrm{H}), 2.92(\mathrm{dd}, J=9.6,1.9 \mathrm{~Hz}, 1 \mathrm{H})$, 2.68-2.60 (m, 2 H), 2.38 (dd, $J=15.9,6.0 \mathrm{~Hz}, 1 \mathrm{H}), 2.29-2.21$ (m, 3H), 2.18-2.08 (m, 2 H), 2.08-2.06 (m, 1 H), 2.03-1.92 (m, 3 H), 1.90-1.82 (m, 2 H), 1.82-1.75 (m, 4H), 1.74-1.57 (m, 6H), 1.53-1.45 (m, $1 \mathrm{H})$, $1.42-1.34(\mathrm{~m}, 2 \mathrm{H}), 1.18(\mathrm{~d}, J=6.8 \mathrm{~Hz}, 3 \mathrm{H}), 1.07(\mathrm{~d}, J=6.4 \mathrm{~Hz}, 3 \mathrm{H}), 1.05-1.02(\mathrm{~m}, 1 \mathrm{H}), 0.89(\mathrm{~s}, 9 \mathrm{H}), 0.87$ (s, 9H), $0.08-0.08(\mathrm{~m}, 12 \mathrm{H}) \mathrm{ppm} ;{ }^{13} \mathrm{C} \mathrm{NMR}\left(151 \mathrm{MHz}, \mathrm{CDCl}_{3}\right): \delta 171.7,159.3,151.8,151.2,130.8$, $129.60,129.57,113.9,110.5,104.9,104.7,82.5,82.2,81.1,79.4,78.6,78.1,77.7,76.7,75.5,74.6,74.5$, 74.1, 73.7, 73.5, 72.2, 68.4, 68.3, 66.6, 59.9, 55.4, 51.8, 47.1, 43.5, 40.6, 40.5, 39.7, 39.0, 36.2, 35.0, 34.7, $34.3,33.7,31.7,30.7,30.1,29.85,29.80,26.1,26.0,18.5,18.4,18.2,16.6,-4.2,-4.7,-5.1,-5.2 \mathrm{ppm}$; HRMS (ESI-TOF) $m / z$ : [M+Na] ${ }^{+}$Calcd. for $\mathrm{C}_{63} \mathrm{H}_{100} \mathrm{O}_{15} \mathrm{Si}_{2} \mathrm{Na}^{+} 1175.6493$; Found 1175.6481. The data is in accordance with Phillips' intermediate. ${ }^{6}$

\section{Condition B:}

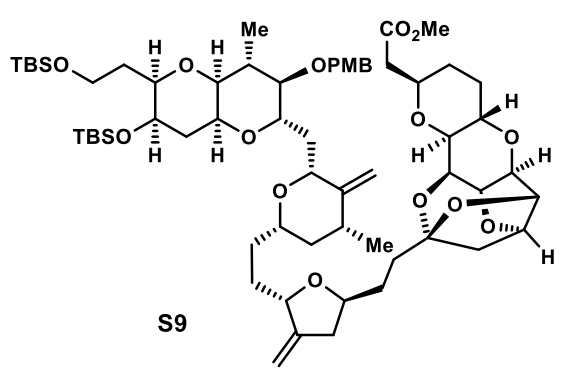

To a stirred solution of 50a $(5.0 \mathrm{mg}, 0.0040 \mathrm{mmol}, 1.0$ equiv) in toluene/ $\mathrm{MeOH}(1.5 \mathrm{~mL}, 10: 1, v / v)$ at $23^{\circ} \mathrm{C}$ were added a premixed solution of $\mathrm{K}_{2} \mathrm{CO}_{3} / 18$-crown-6 $\left(\mathrm{K}_{2} \mathrm{CO}_{3}: 1.7 \mathrm{mg}, 0.012 \mathrm{mmol}, 3.0\right.$ equiv; 18-crown-6: $3.2 \mathrm{mg}, 0.012 \mathrm{mmol}, 3.0$ equiv) in toluene/ $\mathrm{MeOH}(0.1 \mathrm{~mL}, 10: 1, v / v)$. The resulting mixture was allowed to warm to $60^{\circ} \mathrm{C}$ and stirred for $0.5 \mathrm{~h}$ before it was cooled to $0^{\circ} \mathrm{C}$ and quenched by the addition of sat. aq. $\mathrm{NH}_{4} \mathrm{Cl}$ solution $(5 \mathrm{~mL})$. The layers were separated, and the aqueous layer was extracted with EtOAc $(3 \times 10 \mathrm{~mL})$. The combined organic extracts were washed with brine $(5 \mathrm{~mL})$, dried over $\mathrm{Na}_{2} \mathrm{SO}_{4}$, filtered, and concentrated under reduced pressure. Flash column chromatography $\left(\mathrm{SiO}_{2}\right.$, hexanes/EtOAc $3: 1, v / v \rightarrow 1: 1, v / v)$ of the residue afforded $\mathbf{S} 9(2.8 \mathrm{mg}, 0.0024 \mathrm{mmol}, 61 \%$ yield $)$ as a colorless oil. 
NHK Reaction Toward 50b:

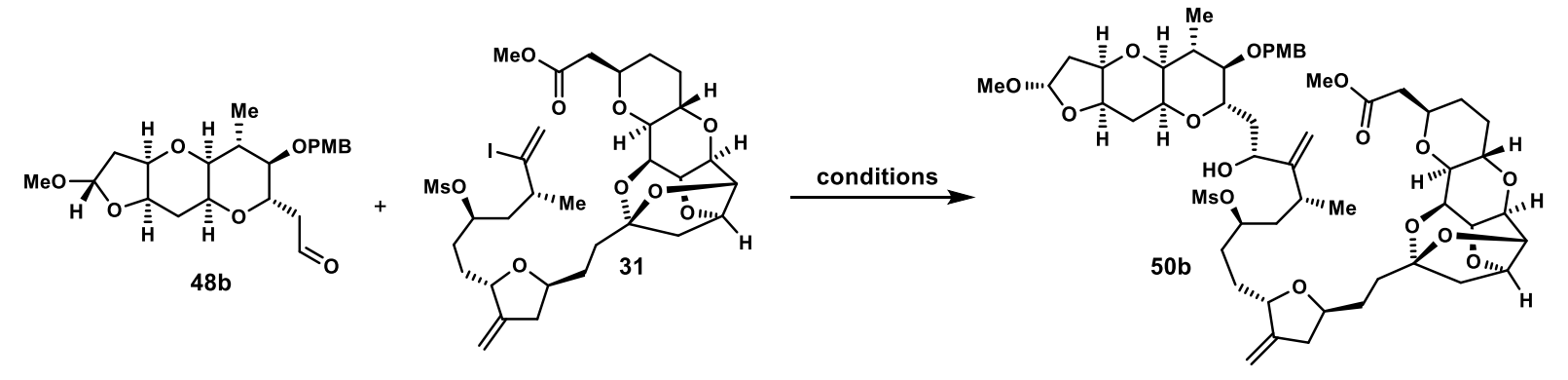

\section{Condition 1:}

Methyl $[(1 S, 2 S, 4 R, 7 S, 9 R, 10 S, 12 S, 14 R, 16 R)-12-\{2-[(2 S, 5 S)-5-\{(3 R, 5 R, 7 R)-7-\mathrm{hyd} \operatorname{roxy}-8$ $\{(2 S, 3 \mathrm{a} R, 4 \mathrm{a} S, 5 R, 6 R, 7 S, 8 \mathrm{a} S, 9 \mathrm{a} R)-2-m e t h o x y-6-[(4-m e t h o x y b e n z y l) 0 x y]-5-m e t h y l d e c a h y d r o f u r o[3,2-$ b]pyrano[2,3-e]pyran-7-yl\}-5-methyl-6-methylidene-3-[(methylsulfonyl)oxy]octyl\}-4-methylidenetetrahydrofuran-2-yl]ethyl $\}-3,8,11,15,17$-pentaoxapentacyclo $\left[10.4 .1 .0^{2,7} \cdot 0^{9,16} \cdot 0^{10,14}\right]$ heptadec-4yl]acetate $(50 \mathrm{~b})$ and methyl $[(1 S, 2 S, 4 R, 7 S, 9 R, 10 S, 12 S, 14 R, 16 R)-12-\{2-[(2 S, 5 S)-5-\{(3 R, 5 R, 7 S)-7-$ hydroxy-8- $\{(2 S, 3 \mathrm{a} R, 4 \mathrm{a} S, 5 R, 6 R, 7 S, 8 \mathrm{a} S, 9 \mathrm{a} R)-2$-methoxy-6-[(4-methoxybenzyl)oxy]-5-methyldecahydrofuro[3,2-b]pyrano[2,3-e]pyran-7-yl\}-5-methyl-6-methylidene-3-[(methylsulfonyl)oxy]octyl\}-4methylidenetetrahydrofuran-2-yl] ethyl $\}-3,8,11,15,17$-pentaoxapentacyclo $\left[10.4 \cdot 1.0^{2,7} \cdot 0^{9,16} \cdot 0^{10,14}\right]$ heptadec-4-yl]acetate (C12-epi-50b): In a glove box, to a stirred solution of $\mathbf{4 8 b}(24.7 \mathrm{mg}, 0.0608 \mathrm{mmol}$,
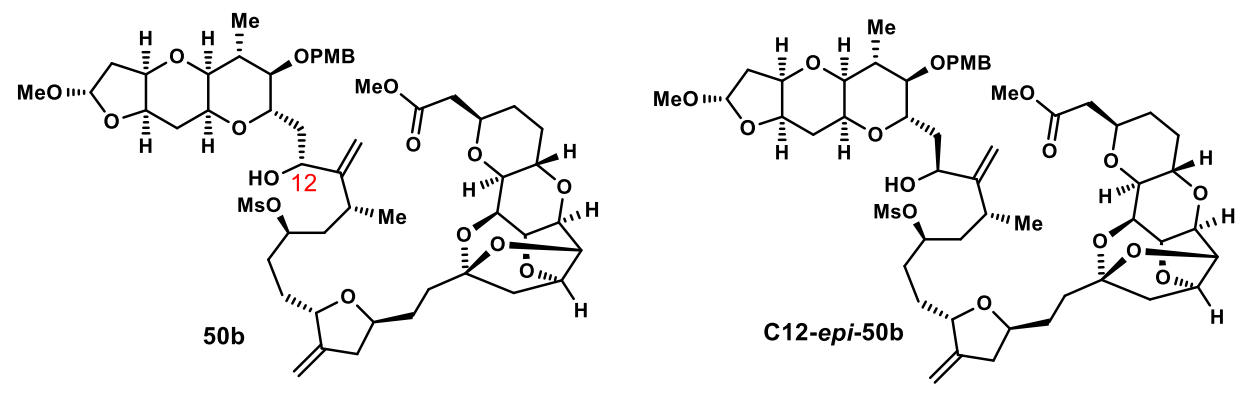

2.0 equiv) and $\mathbf{3 1}$

(22.9 mg, $0.0304 \mathrm{mmol}$,

1.0 equiv) in THF/DMF $(1.0 \mathrm{~mL}, 4: 1, v / v)$ at $23^{\circ} \mathrm{C}$ was added $2.0 \%$

$\mathrm{NiCl}_{2} / \mathrm{CrCl}_{2}$ (37.4mg, $0.304 \mathrm{mmol}, 10.0$ equiv). The resulting mixture was vigorously stirred for $24 \mathrm{~h}$ before it was quenched by the addition of $\mathrm{H}_{2} \mathrm{O}(10 \mathrm{~mL})$. The layers were separated, and the aqueous layer was extracted with EtOAc $(4 \times 30 \mathrm{~mL})$. The organic layer was washed with brine $(10 \mathrm{~mL})$, dried over $\mathrm{Na}_{2} \mathrm{SO}_{4}$, filtered, and concentrated under reduced pressure. Flash column chromatography $\left(\mathrm{SiO}_{2}\right.$, hexanes/EtOAc hexanes/EtOAc 3:1, v/v $\rightarrow 1: 3, v / v)$ of the residue afforded alcohol 50b $(13.5 \mathrm{mg}, 0.0131 \mathrm{mmol}, 43 \%$ yield, 1:1 $d r$ at C12) as a colorless oil, de-I-31 (10.0 mg, $0.0152 \mathrm{mmol}, 50 \%)$ as a colorless oil, and recovered 
aldehyde 48a (13.6mg, $0.0334 \mathrm{mmol}, 55 \%)$ as a white foam. Analytical samples were obtained by preparative TLC. 50b (desired isomer): $\mathrm{R}_{\mathrm{f}}=0.60\left(\mathrm{SiO}_{2}\right.$, hexanes/EtOAc 1:4, v/v); $[\alpha]_{\mathrm{D}}^{23}=-38.7(c=0.41$, $\mathrm{CHCl}_{3}$ ); FT-IR (film): $v_{\max } 3477,2936,2878,1737,1613,1514,1438,1336,1301,1249,1211,1171,1133$, 1074, 1039, 903, 828, $758 \mathrm{~cm}^{-1} ;{ }^{1} \mathrm{H}$ NMR $\left(600 \mathrm{MHz}, \mathrm{C}_{6} \mathrm{D}_{6}\right): \delta 7.29(\mathrm{~d}, J=8.6 \mathrm{~Hz}, 2 \mathrm{H}), 6.85(\mathrm{~d}, J=8.6 \mathrm{~Hz}$, 2H), $5.44(\mathrm{~d}, J=1.3 \mathrm{~Hz}, 1 \mathrm{H}), 5.08(\mathrm{dd}, J=5.4,2.7 \mathrm{~Hz}, 1 \mathrm{H}), 5.03-4.96(\mathrm{~m}, 1 \mathrm{H}), 4.91(\mathrm{~s}, 2 \mathrm{H}), 4.83(\mathrm{~d}$, $J=2.2 \mathrm{~Hz}, 1 \mathrm{H}), 4.60(\mathrm{dd}, J=9.5,2.7 \mathrm{~Hz}, 1 \mathrm{H}), 4.55-4.44(\mathrm{~m}, 3 \mathrm{H}), 4.41(\mathrm{dd}, J=4.0,1.9 \mathrm{~Hz}, 1 \mathrm{H}), 4.34(\mathrm{~d}$, $J=7.8 \mathrm{~Hz}, 1 \mathrm{H}), 4.20-4.15(\mathrm{~m}, 2 \mathrm{H}), 4.13(\mathrm{t}, J=4.7 \mathrm{~Hz}, 1 \mathrm{H}), 3.99-3.93(\mathrm{~m}, 1 \mathrm{H}), 3.91(\mathrm{dd}, J=6.5,4.5 \mathrm{~Hz}$, $1 \mathrm{H}), 3.78-3.74(\mathrm{~m}, 2 \mathrm{H}), 3.68(\mathrm{dd}, J=6.6,3.9 \mathrm{~Hz}, 1 \mathrm{H}), 3.63(\mathrm{dt}, J=6.5,3.1 \mathrm{~Hz}, 1 \mathrm{H}), 3.51-3.44(\mathrm{~m}, 1 \mathrm{H})$, 3.35 (s, $3 \mathrm{H}), 3.34$ (s, $3 \mathrm{H}), 3.29$ (s, $3 \mathrm{H}), 3.00-2.87$ (m, 2 H), 2.66-2.62 (m, $1 \mathrm{H}), 2.62-2.55$ (m, $2 \mathrm{H}), 2.45$ (s, $3 \mathrm{H}), 2.27-2.16(\mathrm{~m}, 4 \mathrm{H}), 2.15-2.03(\mathrm{~m}, 7 \mathrm{H}), 2.01-1.94(\mathrm{~m}, 2 \mathrm{H}), 1.92(\mathrm{dt}, J=14.2,9.8 \mathrm{~Hz}, 1 \mathrm{H}), 1.87-$ $1.73(\mathrm{~m}, 4 \mathrm{H}), 1.70(\mathrm{ddt}, J=17.2,10.4,3.1 \mathrm{~Hz}, 2 \mathrm{H}), 1.65-1.60(\mathrm{~m}, 2 \mathrm{H}), 1.49(\mathrm{dd}, J=13.2,5.1 \mathrm{~Hz}, 1 \mathrm{H})$, 1.44-1.41 (m, $1 \mathrm{H}), 1.34-1.23(\mathrm{~m}, 3 \mathrm{H}), 1.20(\mathrm{~d}, J=6.9 \mathrm{~Hz}, 3 \mathrm{H}), 1.07(\mathrm{~d}, J=7.0 \mathrm{~Hz}, 3 \mathrm{H}) \mathrm{ppm} ;{ }^{1} \mathrm{H}$ NMR $\left(151 \mathrm{MHz}, \mathrm{C}_{6} \mathrm{D}_{6}\right): \delta 171.1,159.9,157.0,152.3,131.2,129.8,114.2,110.4,108.6,105.01,104.96,82.5$, 82.44, 82.38, 81.0, 79.1, 78.6, 77.43, 77.35, 77.0, 75.9, 75.7, 75.5, 75.0, 74.7, 74.33, 74.30, 73.1, 68.5, 65.4, 55.1, 54.9, 51.2, 47.4, 42.0, 40.9, 40.8, 39.34, 39.09, 39.06, 38. 8, 35.8, 32.5, 31.6, 30.9, 30.7, 30.6, 30.3, 29.7, 22.8, 16.9 ppm; HRMS (ESI-TOF) $\mathrm{m} / z$ : $[\mathrm{M}+\mathrm{Na}]^{+}$Calcd. for $\mathrm{C}_{53} \mathrm{H}_{76} \mathrm{O}_{18} \mathrm{SNa}^{+}$1055.4645; Found 1055.4635. C12-epi-50b (undesired isomer): $\mathrm{R}_{\mathrm{f}}=0.60\left(\mathrm{SiO}_{2}\right.$, hexanes/EtOAc 1:4, v/v); ${ }^{1} \mathrm{H}$ NMR $(600 \mathrm{MHz}$, $\left.\mathrm{C}_{6} \mathrm{D}_{6}\right): \delta 7.29(\mathrm{~d}, J=8.5 \mathrm{~Hz}, 2 \mathrm{H}), 6.83(\mathrm{~d}, J=8.6 \mathrm{~Hz}, 2 \mathrm{H}), 5.40(\mathrm{~s}, 1 \mathrm{H}), 5.17(\mathrm{dd}, J=5.7,3.1 \mathrm{~Hz}, 1 \mathrm{H}), 5.10-$ $5.05(\mathrm{~m}, 1 \mathrm{H}), 4.95-4.88(\mathrm{~m}, 2 \mathrm{H}), 4.83(\mathrm{~d}, J=2.1 \mathrm{~Hz}, 1 \mathrm{H}), 4.78(\mathrm{~d}, J=10.8 \mathrm{~Hz}, 1 \mathrm{H}), 4.57-4.45(\mathrm{~m}, 3 \mathrm{H})$, 4.44-4.38 (m, 2 H), $4.36(\mathrm{~d}, J=7.2 \mathrm{~Hz}, 1 \mathrm{H}), 4.18(\mathrm{t}, J=4.5 \mathrm{~Hz}, 1 \mathrm{H}), 4.13(\mathrm{t}, J=4.7 \mathrm{~Hz}, 1 \mathrm{H}), 4.01-3.94(\mathrm{~m}$, $1 \mathrm{H}), 3.94-3.85(\mathrm{~m}, 1 \mathrm{H}), 3.77(\mathrm{ddq}, J=6.7,3.8,2.1 \mathrm{~Hz}, 2 \mathrm{H}), 3.68(\mathrm{dd}, J=6.6,3.9 \mathrm{~Hz}, 1 \mathrm{H}), 3.61(\mathrm{~d}$, $J=3.9 \mathrm{~Hz}, 1 \mathrm{H}), 3.52(\mathrm{td}, J=4.6,1.7 \mathrm{~Hz}, 2 \mathrm{H}), 3.35-3.29(\mathrm{~m}, 9 \mathrm{H}), 3.03(\mathrm{dd}, J=9.0,5.8 \mathrm{~Hz}, 1 \mathrm{H}), 3.00(\mathrm{t}$, $J=4.2 \mathrm{~Hz}, 1 \mathrm{H}), 2.68(\mathrm{dt}, J=9.3,6.3 \mathrm{~Hz}, 1 \mathrm{H}), 2.63-2.55(\mathrm{~m}, 2 \mathrm{H}), 2.45(\mathrm{~s}, 3 \mathrm{H}), 2.31-2.15(\mathrm{~m}, 6 \mathrm{H}), 2.09-$ $2.03(\mathrm{~m}, 3 \mathrm{H}), 2.02-1.94(\mathrm{~m}, 4 \mathrm{H}), 1.88-1.81(\mathrm{~m}, 3 \mathrm{H}), 1.80-1.76(\mathrm{~m}, 2 \mathrm{H}), 1.75-1.71(\mathrm{~m}, 2 \mathrm{H}), 1.71-1.66$ $(\mathrm{m}, 2 \mathrm{H}), 1.56(\mathrm{dt}, J=14.3,4.9 \mathrm{~Hz}, 2 \mathrm{H}), 1.49(\mathrm{dd}, J=13.1,5.0 \mathrm{~Hz}, 2 \mathrm{H}), 1.13(\mathrm{~d}, J=6.9 \mathrm{~Hz}, 3 \mathrm{H}), 1.02(\mathrm{~d}$, $J=7.3 \mathrm{~Hz}, 3 \mathrm{H}) \mathrm{ppm} ;{ }^{13} \mathrm{H}$ NMR $\left(151 \mathrm{MHz}, \mathrm{C}_{6} \mathrm{D}_{6}\right): \delta 171.2,159.8,157.2,152.3,131.4,129.8,114.1,110.5$, $108.9,105.2,105.0,83.2,82.8,82.4,81.0,79.1,78.6,77.3,77.2,77.0,75.7,74.9,74.7,74.3,73.9,72.3$, 
$70.1,69.8,68.5,64.3,55.5,54.8,51.2,47.4,41.5,41.4,40.8,40.7,39.3,39.1,38.7,35.8,32.7,31.7,30.9$, 30.7, 30.6, 30.3, 30.1, 22.8, 17.7 ppm; HRMS (ESI-TOF) $\mathrm{m} / z$ : $[\mathrm{M}+\mathrm{Na}]^{+}$Calcd. for $\mathrm{C}_{53} \mathrm{H}_{76} \mathrm{O}_{18} \mathrm{SNa}^{+}$ 1055.4645; Found 1055.4633.

\section{Condition 4:}

Methyl $[(1 S, 2 S, 4 R, 7 S, 9 R, 10 S, 12 S, 14 R, 16 R)-12-\{2-[(2 S, 5 S)-5-\{(3 R, 5 R, 7 R)-7-\mathrm{hyd} \operatorname{roxy}-8-$ $\{(2 S, 3 \mathrm{a} R, 4 \mathrm{a} S, 5 R, 6 R, 7 S, 8 \mathrm{a} S, 9 \mathrm{a} R)-2-$ methoxy-6-[(4-methoxybenzyl)oxy]-5-methyldecahydrofuro[3,2b]pyrano[2,3-e]pyran-7-yl\}-5-methyl-6-methylidene-3-[(methylsulfonyl)oxy]octyl\}-4-methylidenetetrahydrofuran-2-yl] ethyl\}-3,8,11,15,17-pentaoxapentacyclo[10.4.1.0 $\left.{ }^{2,7} \cdot 0^{9,16} \cdot 0^{10,14}\right]$ heptadec-4-yl]acetate (50b): In a glove box, to a stirred solution of $\mathbf{4 8 b}(15 \mathrm{mg}, 0.036 \mathrm{mmol}, 1.2$ equiv), 31 (23 $\mathrm{mg}$,

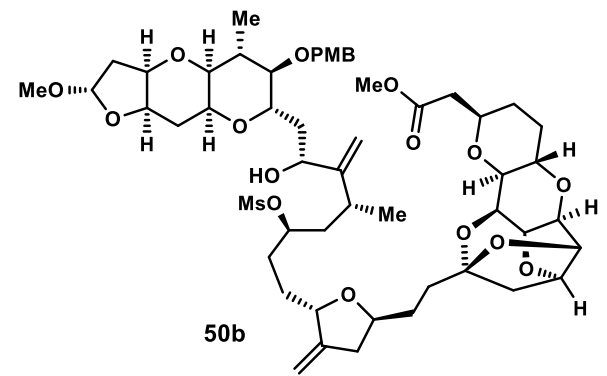
$0.031 \mathrm{mmol}, 1.0$ equiv), $\mathrm{Et}_{3} \mathrm{~N}(21 \mu \mathrm{L}, 0.15 \mathrm{mmol}, 5.0$ equiv) and $\mathrm{CrCl}_{2}\left(11 \mathrm{mg}, 0.090 \mathrm{mmol}, 3.0\right.$ equiv) in THF $(0.5 \mathrm{~mL})$ at $23^{\circ} \mathrm{C}$ was added a premixed solution of ligand $49(45 \mathrm{mg}, 0.15 \mathrm{mmol}$, 2.5 equiv) and $\mathrm{NiCl}_{2}(1.0 \mathrm{mg}, 0.0075 \mathrm{mmol}, 0.25$ equiv) in $\mathrm{THF}$ $(0.5 \mathrm{~mL})$. The resulting mixture was stirred for $6 \mathrm{~h}$ before it was quenched by the addition of $\mathrm{H}_{2} \mathrm{O}(10 \mathrm{~mL})$. The layers were separated, and the aqueous layer was extracted with EtOAc $(4 \times 30 \mathrm{~mL})$. The organic layer was washed with brine $(10 \mathrm{~mL})$, dried over $\mathrm{Na}_{2} \mathrm{SO}_{4}$, filtered, and concentrated under reduced pressure. Flash column chromatography $\left(\mathrm{SiO}_{2}\right.$, hexanes/EtOAc hexanes/ EtOAc $3: 1, v / v \rightarrow 1: 3, v / v)$ of the residue afforded 50b $(19 \mathrm{mg}, 0.019 \mathrm{mmol}, 62 \%$ yield, $d r>5: 1)$ as a colorless oil. 
Cyclization Toward Methyl [(1S,2S,4R,7S,9R,10S,12S,14R,16R)-12-\{2-[(2S,5S)-5-\{2-[(2S,4R,6R)-6(\{(2S,3a $R, 4 \mathrm{a} S, 5 R, 6 R, 7 S, 8 \mathrm{a} S, 9 \mathrm{a} R)-2-M e t h o x y-6-[(4-m e t h o x y b e n z y l) o x y]-5-m e t h y l d e c a h y d r o f u r o-$ [3,2-b]pyrano[2,3-e]pyran-7-yl\} methyl)-4-methyl-5-methylidenetetrahydro-2H-pyran-2-yl]ethyl\}-4methylidenetetrahydrofuran-2-yl]ethyl $\}-3,8,11,15,17$-pentaoxapentacyclo $\left[10.4 .1 .0^{2,7} \cdot 0^{9,16} \cdot 0^{10,14}\right]-$ heptadec-4-yl]acetate (S10):

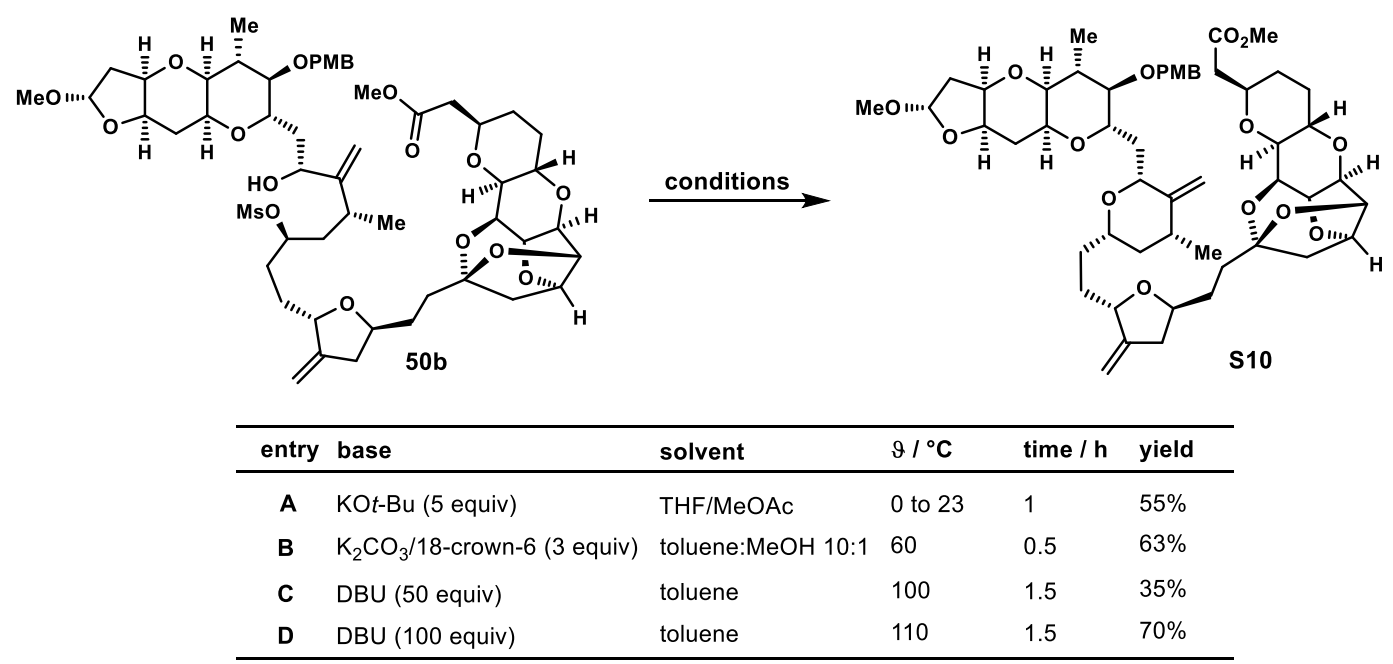

\section{Condition D:}

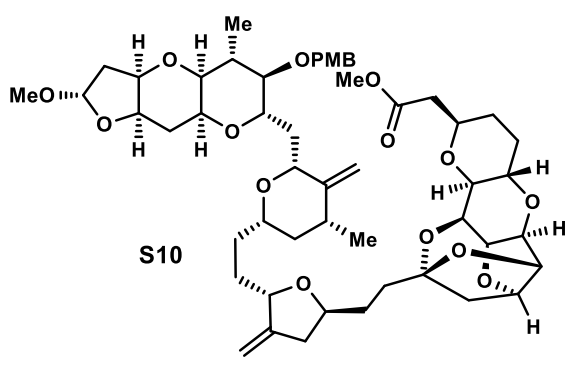

To a stirred solution of $\mathbf{5 0 b}(10 \mathrm{mg}, 0.0097 \mathrm{mmol}, 1.0$ equiv) in toluene $(2 \mathrm{~mL})$ at $23^{\circ} \mathrm{C}$ were added DBU $(0.15 \mathrm{~mL}, 0.97 \mathrm{mmol}$, 1.0 equiv). The resulting mixture was warmed to $110^{\circ} \mathrm{C}$ and stirred for $1.5 \mathrm{~h}$ before it was allowed to cool to $23^{\circ} \mathrm{C}$ and quenched by the addition of sat. aq. $\mathrm{NH}_{4} \mathrm{Cl}$ solution $(5 \mathrm{~mL})$. The layers were separated, and the aqueous layer was extracted with EtOAc $(3 \times 10 \mathrm{~mL})$. The combined organic extracts were washed with brine $(5 \mathrm{~mL})$, dried over $\mathrm{Na}_{2} \mathrm{SO}_{4}$, filtered, and concentrated under reduced pressure. Flash column chromatography $\left(\mathrm{SiO}_{2}\right.$, hexanes/EtOAc 3:1, v/v $\left.\rightarrow 1: 2, v / v\right)$ of the residue afforded $\mathbf{S 1 0}(6.3 \mathrm{mg}$, $0.0068 \mathrm{mmol}, 70 \%$ yield $)$ as a colorless oil. S10: $\mathrm{R}_{\mathrm{f}}=0.80\left(\mathrm{SiO}_{2}, 100 \%\right.$ EtOAc $) ;[\alpha]_{\mathrm{D}}^{23}=-32.6(c=0.60$, EtOAc); FT-IR (film): $v_{\max } 2928,2870,1738,1612,1514,1438,1354,1301,1248,1210,1188,1154,1133$, 1076, 1039, 997, 907, 824, 731 cm ${ }^{-1} ;{ }^{1} \mathrm{H}$ NMR $\left(600 \mathrm{MHz}, \mathrm{CDCl}_{3}\right): \delta 7.26(\mathrm{~d}, J=8.6 \mathrm{~Hz}, 2 \mathrm{H}), 6.86(\mathrm{~d}$, $J=8.6 \mathrm{~Hz}, 2 \mathrm{H}), 5.05(\mathrm{dd}, J=5.4,2.0 \mathrm{~Hz}, 1 \mathrm{H}), 4.97(\mathrm{~d}, J=2.1 \mathrm{~Hz}, 1 \mathrm{H}), 4.93(\mathrm{~s}, 1 \mathrm{H}), 4.84(\mathrm{~d}, J=2.1 \mathrm{~Hz}, 1 \mathrm{H})$, $4.80(\mathrm{~d}, J=1.9 \mathrm{~Hz}, 1 \mathrm{H}), 4.68(\mathrm{t}, J=4.7 \mathrm{~Hz}, 1 \mathrm{H}), 4.60(\mathrm{t}, J=4.6 \mathrm{~Hz}, 1 \mathrm{H}), 4.58-4.48(\mathrm{~m}, 2 \mathrm{H}), 4.43(\mathrm{dd}, J=4.0$, 
$1.9 \mathrm{~Hz}, 1 \mathrm{H}), 4.39$ (s, $1 \mathrm{H}), 4.28(\mathrm{td}, J=10.1,4.4 \mathrm{~Hz}, 1 \mathrm{H}), 4.20(\mathrm{dd}, J=6.6,4.6 \mathrm{~Hz}, 1 \mathrm{H}), 4.09-4.03(\mathrm{~m}, 2 \mathrm{H})$, 4.05-3.98 (m, 2 H), $3.91(\mathrm{dd}, J=8.3,4.8 \mathrm{~Hz}, 1 \mathrm{H}), 3.84-3.78(\mathrm{~m}, 5 \mathrm{H}), 3.78-3.74(\mathrm{~m}, 1 \mathrm{H}), 3.67(\mathrm{~s}, 3 \mathrm{H})$, 3.62-3.55 (m, $1 \mathrm{H}), 3.32-3.30(\mathrm{~m}, 4 \mathrm{H}), 2.99(\mathrm{t}, J=8.4 \mathrm{~Hz}, 1 \mathrm{H}), 2.92(\mathrm{dd}, J=9.5,1.9 \mathrm{~Hz}, 1 \mathrm{H}), 2.65(\mathrm{dd}$, $J=15.9,6.8 \mathrm{~Hz}, 2 \mathrm{H}), 2.39(\mathrm{dd}, J=15.9,6.0 \mathrm{~Hz}, 1 \mathrm{H}), 2.31-2.21(\mathrm{~m}, 3 \mathrm{H}), 2.17-2.05(\mathrm{~m}, 5 \mathrm{H}), 1.97(\mathrm{tt}$, $J=14.8,7.1 \mathrm{~Hz}, 4 \mathrm{H}), 1.87-1.75$ (m, 4H), 1.74-1.60 (m, 6H), 1.51-1.47 (m, 1 H), 1.42-1.33 (m, $2 \mathrm{H}), 1.14$ $(\mathrm{d}, J=6.8 \mathrm{~Hz}, 3 \mathrm{H}), 1.07$ (d, $J=6.4 \mathrm{~Hz}, 3 \mathrm{H}) \mathrm{ppm} ;{ }^{13} \mathrm{C} \mathrm{NMR}\left(151 \mathrm{MHz}, \mathrm{CDCl}_{3}\right): \delta 171.7,159.4,151.9,151.14$, $130.6,129.6,114.0,110.5,104.9,104.8,104.6,82.5,82.2,81.2,79.5,78.2,77.8,77.5,76.8,75.6,75.2$ $74.9,74.59,74.55,74.1,73.3,72.3,68.4,66.0,55.4,55.0,51.8,47.1,43.6,40.6,40.4,39.0,38.8,36.2$, 35.1, 35.0, 31.72, 31.67, 30.7, 30.1, 29.8, 29.0, 18.2, 16.4 ppm; HRMS (ESI-TOF) $m / z$ : $[\mathrm{M}+\mathrm{Na}]^{+}$Calcd. for $\mathrm{C}_{52} \mathrm{H}_{72} \mathrm{O}_{15} \mathrm{Na}^{+}$959.4763; Found 959.4757.

Methyl $[(1 S, 2 S, 4 R, 7 S, 9 R, 10 S, 12 S, 14 R, 16 R)-12-\{2-[(2 S, 5 S)-5-\{(3 R, 5 R, 7 R)-7-\mathrm{hyd}$ roxy-8$\left[\left(2 R, 2^{\prime \prime} R, 3 \mathrm{a} R, 3 \mathrm{a}^{\prime} S, 3 \mathrm{a}^{\prime \prime} R, 4 \mathrm{a} S, 4 \mathrm{a}^{\prime \prime} S, 5 R, 5^{\prime} R, 5^{\prime \prime} S, 6 R, 7 S, 7^{\prime} S, 7 \mathrm{a}^{\prime} S, 8 \mathrm{a} S, 8 \mathrm{a}^{\prime \prime} S, 9 \mathrm{a} R, 9 \mathrm{a}^{\prime \prime} R\right)-6-\mathrm{hydroxy} \mathbf{2}^{\prime \prime}-\right.$ methoxy-5,5", 7'-trimethylicosahydro-2" $\mathrm{H,3H}, 3^{\prime} \mathrm{H}$-dispiro[furo[3,2-b]pyrano[2,3-e]pyran-2,2'furo[3,2-b]pyran-5',7"-furo[3,2-b]pyrano[2,3-e]pyran]-7-yl]-5-methyl-6-methylidene-3-[(methylsulfonyl)oxy]octyl\}-4-methylidenetetrahydrofuran-2-yl]ethyl\}-3,8,11,15,17-pentaoxapentacyclo$\left[10.4 .1 .0^{2,7} \cdot 0^{9,16} \cdot 0^{10,14}\right]$ heptadec-4-yl] acetate (51): In a glove box, to a stirred solution of C16-epi-30

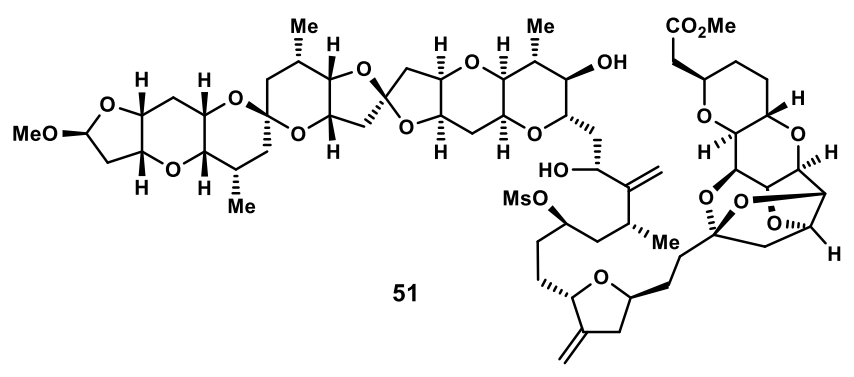
(20 mg, $0.034 \mathrm{mmol}, \quad 1.0$ equiv), 31 (31 mg, $0.041 \mathrm{mmol}, 1.2$ equiv), $\mathrm{Et}_{3} \mathrm{~N}(29 \mu \mathrm{L}, 0.20 \mathrm{mmol}$, 6.0 equiv), and $\mathrm{CrCl}_{2} \quad(15 \mathrm{mg}, \quad 0.12 \mathrm{mmol}$, 3.6 equiv) in THF $(0.2 \mathrm{~mL})$ at $23^{\circ} \mathrm{C}$ was added a premixed solution of ligand 49 (31 mg, $0.10 \mathrm{mmol}, 3.0$ equiv) and $\mathrm{NiCl}_{2}(1.1 \mathrm{mg}, 0.0082 \mathrm{mmol}, 0.2$ equiv) in THF $(0.5 \mathrm{~mL})$. The resulting mixture was stirred for $6 \mathrm{~h}$ before it was quenched by the addition of $\mathrm{H}_{2} \mathrm{O}(10 \mathrm{~mL})$. The layers were separated, and the aqueous layer was extracted with EtOAc $(4 \times 30 \mathrm{~mL})$. The organic layer was washed with brine $(10 \mathrm{~mL})$, dried over $\mathrm{Na}_{2} \mathrm{SO}_{4}$, filtered, and concentrated under reduced pressure. Flash column chromatography $\left(\mathrm{SiO}_{2}\right.$, hexanes/EtOAc 3:1, v/v $\rightarrow 1: 3, v / v)$ of the residue afforded $51(27 \mathrm{mg}, 0.022 \mathrm{mmol}, 65 \%$ yield) as a light 
green oil. 51: $\mathrm{R}_{\mathrm{f}}=0.40\left(\mathrm{SiO}_{2}, 100 \%\right.$ EtOAc); $[\alpha]_{\mathrm{D}}^{23}=-36.0\left(c=0.10\right.$, EtOAc); FT-IR (film): $v_{\max } 3477,2926$, $2872,1736,1555,1436,1333,1264,1194,1171,1076,1019,973,904,846 \mathrm{~cm}^{-1}$; ${ }^{1} \mathrm{H}$ NMR $(151 \mathrm{MHz}$, $\left.\mathrm{C}_{6} \mathrm{D}_{6}\right): \delta 5.33(\mathrm{~s}, 1 \mathrm{H}), 5.15(\mathrm{dd}, J=5.8,3.7 \mathrm{~Hz}, 1 \mathrm{H}), 4.99-4.93(\mathrm{~m}, 1 \mathrm{H}), 4.93(\mathrm{~d}, J=2.2 \mathrm{~Hz}, 1 \mathrm{H}), 4.88(\mathrm{~s}$, $1 \mathrm{H}), 4.83(\mathrm{~d}, J=2.2 \mathrm{~Hz}, 1 \mathrm{H}), 4.58-4.45(\mathrm{~m}, 1 \mathrm{H}), 4.41(\mathrm{dd}, J=4.0,1.9 \mathrm{~Hz}, 1 \mathrm{H}), 4.39-4.32(\mathrm{~m}, 1 \mathrm{H}), 4.19(\mathrm{t}$, $J=4.5 \mathrm{~Hz}, 1 \mathrm{H}), 4.14(\mathrm{t}, J=4.7 \mathrm{~Hz}, 1 \mathrm{H}), 4.06-4.00(\mathrm{~m}, 1 \mathrm{H}), 4.01-3.95(\mathrm{~m}, 1 \mathrm{H}), 3.93-3.90(\mathrm{~m}, 1 \mathrm{H}), 3.90-$ $3.87(\mathrm{~m}, 1 \mathrm{H}), 3.82(\mathrm{dd}, J=4.7,2.2 \mathrm{~Hz}, 1 \mathrm{H}), 3.78-3.72(\mathrm{~m}, 4 \mathrm{H}), 3.68(\mathrm{dd}, J=6.6,3.9 \mathrm{~Hz}, 1 \mathrm{H}), 3.58(\mathrm{dd}$, $J=5.5,2.4 \mathrm{~Hz}, 1 \mathrm{H}), 3.55-3.51(\mathrm{~m}, 1 \mathrm{H}), 3.35(\mathrm{~s}, 3 \mathrm{H}), 3.34-3.31(\mathrm{~m}, 1 \mathrm{H}), 3.20(\mathrm{~s}, 3 \mathrm{H}), 3.10-3.04(\mathrm{~m}, 1 \mathrm{H})$, $3.02(\mathrm{dd}, J=6.7,5.2 \mathrm{~Hz}, 1 \mathrm{H}), 2.69(\mathrm{~d}, J=2.8 \mathrm{~Hz}, 1 \mathrm{H}), 2.63-2.56(\mathrm{~m}, 3 \mathrm{H}), 2.47-2.43(\mathrm{~m}, 2 \mathrm{H}), 2.43(\mathrm{~s}, 3 \mathrm{H})$, 2.40-2.33 (m, $1 \mathrm{H}), 2.33-2.25(\mathrm{~m}, 3 \mathrm{H}), 2.20(\mathrm{dd}, J=15.7,5.3 \mathrm{~Hz}, 1 \mathrm{H}), 2.18-2.12(\mathrm{~m}, 4 \mathrm{H}), 2.08-2.02(\mathrm{~m}$, 4H), 2.02-1.93 (m, 5 H), 1.82-1.67 (m, 7H), 1.54-1.48 (m, 2 H), 1.48-1.40 (m, 4H), 1.35-1.26 (m, 5 H), $1.19(\mathrm{~d}, J=6.9 \mathrm{~Hz}, 3 \mathrm{H}), 1.17(\mathrm{~d}, J=6.8 \mathrm{~Hz}, 3 \mathrm{H}), 1.06(\mathrm{~d}, J=7.1 \mathrm{~Hz}, 3 \mathrm{H}), 1.01(\mathrm{~d}, J=6.9 \mathrm{~Hz}, 3 \mathrm{H}) \mathrm{ppm} ;{ }^{1} \mathrm{H}$ NMR (151 MHz, $\left.\mathrm{C}_{6} \mathrm{D}_{6}\right): \delta 171.2,156.8,152.3,114.8,110.5,109.0,105.0,104.9,97.0,82.6,82.4,81.0$, 79.5, 79.0, 78.6, 77.6, 77.41, 77.36, 77.33, 77.2, 77.00, 76.9, 76.4, 75.0, 74.8, 74.7, 74.3, 74.0, 73.9, 72.3, $72.0171 .5,68.5,66.3,63.8,55.3,51.2,47.4,42.1,41.8,40.8,39.6,39.1,38.9,38.7,37.7,37.2,35.8,32.4$, $31.5,30.9,30.7,30.6,30.5,30.3,29.2,26.1,22.8,18.1,17.5,16.7,15.7 \mathrm{ppm}$; HRMS (ESI-TOF) $\mathrm{m} / \mathrm{z}$ : $[\mathrm{M}+\mathrm{Na}]^{+}$Calcd. for $\mathrm{C}_{62} \mathrm{H}_{92} \mathrm{O}_{22} \mathrm{SNa}^{+}$1243.5693; Found 1243.5697.

Methyl [ $(1 S, 2 S, 4 R, 7 S, 9 R, 10 S, 12 S, 14 R, 16 R)-12-\{2-[(2 S, 5 S)-5-\{2-[(2 S, 4 R, 6 R)-6-$ $\left\{\left[\left(2 R, 2^{\prime \prime} R, 3 \mathrm{a} R, 3 \mathrm{a}^{\prime} S, 3 \mathrm{a}^{\prime \prime} R, 4 \mathrm{a} S, 4 \mathrm{a}^{\prime \prime} S, 5 R, 5^{\prime} R, 5^{\prime \prime} S, 6 R, 7 S, 7^{\prime} S, 7 \mathrm{a}^{\prime} S, 8 \mathrm{a} S, 8 \mathrm{a}^{\prime \prime} S, 9 \mathrm{a} R, 9 \mathrm{a}^{\prime \prime} R\right)-6-\mathrm{hydroxy}-2^{\prime \prime}-\right.\right.$ methoxy-5,5", 7'-trimethylicosahydro-2" $H, 3 H, 3 ' H$-dispiro[furo[3,2-b]pyrano[2,3-e]pyran-2,2'-furo[3,2-b]pyran-5',7"-furo[3,2-b]pyrano[2,3-e]pyran]-7-yl]methyl\}-4-methyl-5-methylidenetetrahydro2H-pyran-2-yl]ethyl\}-4-methylidenetetrahydrofuran-2-yl]ethyl\}-3,8,11,15,17-pentaoxapentacyclo-

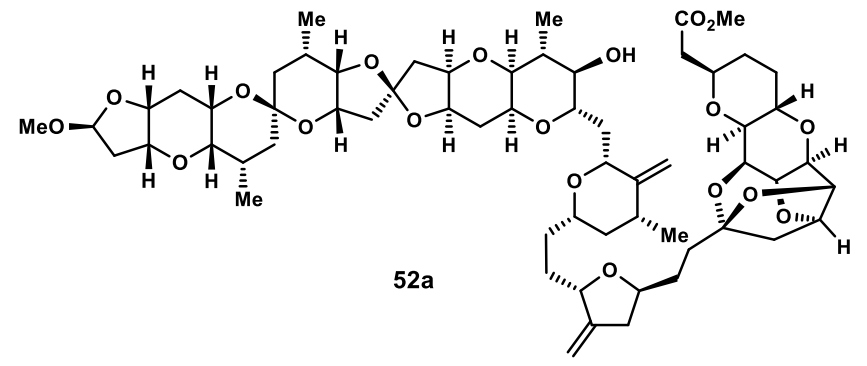
$\left[10.4 .1 .0^{2,7} \cdot 0^{9,16} \cdot 0^{10,14}\right]$ heptadec-4-yl]acetate (52a): To a stirred solution of $\mathbf{5 1}(10 \mathrm{mg}$, $\begin{array}{llllllllllll}0 & . & 0 & 0 & 8 & 2 & \mathrm{~m} & \mathrm{~m} & \mathrm{o} & 1\end{array}$, 1.0 equiv) in toluene $(1.5 \mathrm{~mL})$ at $23^{\circ} \mathrm{C}$ was added DBU $(0.13 \mathrm{~mL}, 0.82 \mathrm{mmol}, 100$ equiv $)$. The 
resulting mixture was warmed to $110^{\circ} \mathrm{C}$ and stirred for $1 \mathrm{~h}$ before it was allowed to cool to $23^{\circ} \mathrm{C}$ and quenched by the addition of sat. aq. $\mathrm{NH}_{4} \mathrm{Cl}$ solution $(5 \mathrm{~mL})$. The layers were separated, and the aqueous layer was extracted with EtOAc $(3 \times 10 \mathrm{~mL})$. The combined organic extracts were washed with brine $(5 \mathrm{~mL})$, dried over $\mathrm{Na}_{2} \mathrm{SO}_{4}$, filtered, and concentrated under reduced pressure. Flash column chromatography $\left(\mathrm{SiO}_{2}\right.$, hexanes/EtOAc 2:1, v/v $\rightarrow 1: 5, v / v)$ of the residue afforded 52a $(6.5 \mathrm{mg}, 0.0057 \mathrm{mmol}, 70 \%$ yield $)$ as a colorless oil. 52a: $\mathrm{R}_{\mathrm{f}}=0.60\left(\mathrm{SiO}_{2}, 100 \%\right.$ EtOAc); $[\alpha]_{\mathrm{D}}^{23}=-36.7\left(c=0.10\right.$, EtOAc); FT-IR (film): $v_{\max } 3493$, 2925, 2870, 1739, 1553, 1457, 1330, 1261, 1193, 1078, 1021, 900,802 $\mathrm{cm}^{-1} .{ }^{1} \mathrm{H}$ NMR $\left(600 \mathrm{MHz}, \mathrm{C}_{6} \mathrm{D}_{6}\right): \delta$ $5.16(\mathrm{dd}, J=5.9,3.6 \mathrm{~Hz}, 1 \mathrm{H}), 4.99-4.91(\mathrm{~m}, 3 \mathrm{H}), 4.79(\mathrm{~d}, J=1.8 \mathrm{~Hz}, 1 \mathrm{H}), 4.56-4.50(\mathrm{~m}, 2 \mathrm{H}), 4.43$ (dd, $J=3.9,1.9 \mathrm{~Hz}, 1 \mathrm{H}), 4.17(\mathrm{t}, J=4.6 \mathrm{~Hz}, 1 \mathrm{H}), 4.11(\mathrm{t}, J=4.8 \mathrm{~Hz}, 1 \mathrm{H}), 4.08(\mathrm{t}, J=5.7 \mathrm{~Hz}, 1 \mathrm{H}), 4.03(\mathrm{t}$, $J=6.7 \mathrm{~Hz}, 1 \mathrm{H}), 3.91(\mathrm{ddd}, J=13.1,6.9,4.7 \mathrm{~Hz}, 3 \mathrm{H}), 3.87(\mathrm{t}, J=2.6 \mathrm{~Hz}, 1 \mathrm{H}), 3.81(\mathrm{dd}, J=4.8,2.0 \mathrm{~Hz}, 1 \mathrm{H})$, $3.76(\mathrm{dd}, J=5.1,2.4 \mathrm{~Hz}, 2 \mathrm{H}), 3.72-3.67(\mathrm{~m}, 2 \mathrm{H}), 3.66-3.61(\mathrm{~m}, 1 \mathrm{H}), 3.57(\mathrm{dd}, J=5.5,2.3 \mathrm{~Hz}, 1 \mathrm{H}), 3.48-$ 3.37 (m, $1 \mathrm{H}), 3.39-3.30(\mathrm{~m}, 6 \mathrm{H}), 3.25-3.18(\mathrm{~m}, 4 \mathrm{H}), 2.69$ (d, J=2.8 Hz, $1 \mathrm{H}), 2.62-2.53$ (m, $3 \mathrm{H}), 2.53-$ $2.42(\mathrm{~m}, 3 \mathrm{H}), 2.38-2.28(\mathrm{~m}, 3 \mathrm{H}), 2.26-2.10(\mathrm{~m}, 8 \mathrm{H}), 2.10-2.02(\mathrm{~m}, 3 \mathrm{H}), 2.02-1.92(\mathrm{~m}, 4 \mathrm{H}), 1.90-1.83$ (m, $1 \mathrm{H}), 1.82-1.73(\mathrm{~m}, 4 \mathrm{H}), 1.71-1.66(\mathrm{~m}, 1 \mathrm{H}), 1.55-1.45(\mathrm{~m}, 5 \mathrm{H}), 1.40(\mathrm{~d}, J=12.5 \mathrm{~Hz}, 3 \mathrm{H}), 1.35-1.27$ $(\mathrm{m}, 4 \mathrm{H}), 1.10-1.08(\mathrm{~m}, 1 \mathrm{H}), 1.05(\mathrm{~d}, J=7.0 \mathrm{~Hz}, 3 \mathrm{H}), 1.01(\mathrm{~d}, J=6.8 \mathrm{~Hz}, 3 \mathrm{H}), 0.93(\mathrm{~d}, J=6.5 \mathrm{~Hz}, 3 \mathrm{H}) \mathrm{ppm}$; ${ }^{13} \mathrm{C}$ NMR $\left(151 \mathrm{MHz}, \mathrm{C}_{6} \mathrm{D}_{6}\right): \delta 171.2,152.8,151.4,127.6,114.7,110.5,104.9,104.8,97.0,82.3,81.0,79.4$, $79.2,78.7,78.5,77.9,77.3,77.0,76.9,76.1,75.7,75.0,74.7,74.42,74.36,73.9,73.1,72.3,72.1,68.5$, 67.7, 63.8, 55.3, 51.1, 47.3, 45.6, 45.4, 43.1, 42.1, 40.7, 40.0, 39.3, 37.7, 37.2, 36.2, 35.9, 34.2, 32.1, 31.9, 30.9, 30.7, 30.6, 30.5, 30.3, 30.1, 29.2, 26.1, 18.2, 18.0, 17.5, $15.8 \mathrm{ppm}$; HRMS (ESI-TOF) $\mathrm{m} / \mathrm{z}:[\mathrm{M}+\mathrm{Na}]^{+}$ Calcd. for $\mathrm{C}_{61} \mathrm{H}_{88} \mathrm{O}_{19} \mathrm{Na}^{+}$1147.5812; Found 1147.5796. 
$\left[(1 S, 2 S, 4 R, 7 S, 9 R, 10 S, 12 S, 14 R, 16 R)-12-\left\{2-\left[(2 S, 5 S)-5-\left\{2-\left[(2 S, 4 R, 6 R)-6-\left\{\left[\left(2 R, 2^{\prime \prime} R, 3 \mathrm{a} R, 3 \mathrm{a}^{\prime} S, 3 \mathrm{a}^{\prime \prime} R, 4 \mathrm{a} S\right.\right.\right.\right.\right.\right.\right.\right.$, $\left.4 a^{\prime \prime} S, 5 R, 5^{\prime} R, 5^{\prime \prime} S, 6 R, 7 S, 7^{\prime} S, 7 a^{\prime} S, 8 a S, 8 a^{\prime \prime} S, 9 a R, 9 a^{\prime \prime} R\right)-6-H y d r o x y-2 "$-methoxy-5,5",7'-trimethylicosahydro-2" $H, 3 H, 3^{\prime} H$-dispiro[furo[3,2-b]pyrano[2,3-e]pyran-2,2'-furo[3,2-b]pyran-5',7"'-furo[3,2b]pyrano[2,3-e]pyran]-7-yl]methyl\}-4-methyl-5-methylidenetetrahydro-2H-pyran-2-yl]ethyl\}-4methylidenetetrahydrofuran-2-yl] ethyl $\}-3,8,11,15,17$-pentaoxapentacyclo $\left[10.4 .1 .0^{2,7} \cdot 0^{9,16} \cdot 0^{10,14}\right]-$ heptadec-4-yl]acetic acid (52): To a stirred solution of 52a (6.5 mg, $0.0057 \mathrm{mmol}, 1.0$ equiv) in THF/ $\mathrm{H}_{2} \mathrm{O}$

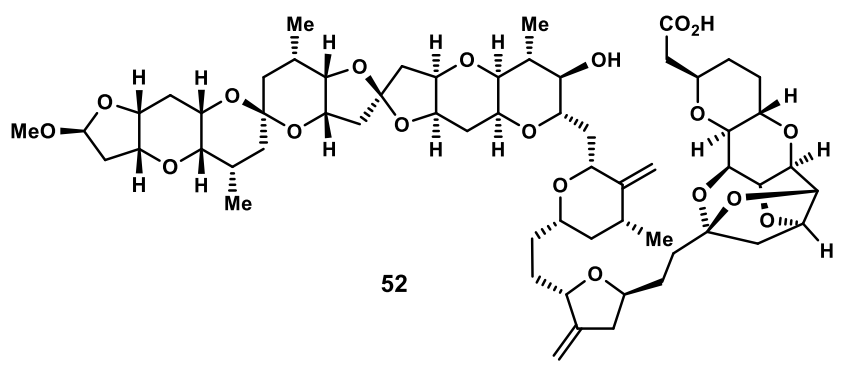
$(1 \mathrm{~mL}, 2: 1, v / v)$ at $23^{\circ} \mathrm{C}$ was added $\mathrm{LiOH}(2.5 \mathrm{mg}$, $0.11 \mathrm{mmol}, 20.0$ equiv). The resulting mixture was stirred for $2 \mathrm{~h}$ before it was quenched by the addition of aq. $\mathrm{HCl}(0.1 \mathrm{M}, 2 \mathrm{~mL})$. The layers were separated, and the aqueous layer was extracted with $\mathrm{CH}_{2} \mathrm{Cl}_{2}(4 \times 10 \mathrm{~mL})$. The combined organic extracts were dried over $\mathrm{Na}_{2} \mathrm{SO}_{4}$, filtered, and

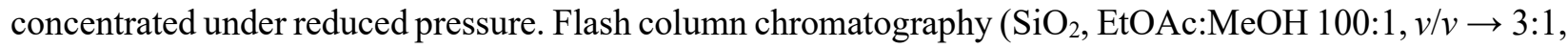
$v / v)$ of the residue afforded carboxylic acid $\mathbf{5 2}(6.3 \mathrm{mg}, 0.0057 \mathrm{mmol}$, quant. yield) as a colorless oil. $\mathbf{5 2}$ : $\mathrm{R}_{\mathrm{f}}=0.60\left(\mathrm{SiO}_{2}, \mathrm{EtOAc} / \mathrm{MeOH} 5: 1, v / v\right) ;[\alpha]_{\mathrm{D}}^{23}=-43.3\left(c=0.15, \mathrm{CHCl}_{3}\right) ;$ FTIR (film): $v_{\max } 3430,2927,2870$, 1732, 1556, 1457, 1370, 1262, 1191, 1131, 1072, 1050, 1021, 972, 895, $828 \mathrm{~cm}^{-1} ;{ }^{1} \mathrm{H}$ NMR (600 MHz, $\left.\mathrm{C}_{6} \mathrm{D}_{6}\right): \delta 5.16(\mathrm{dd}, J=5.8,3.6 \mathrm{~Hz}, 1 \mathrm{H}), 5.00(\mathrm{~s}, 1 \mathrm{H}), 4.97(\mathrm{~d}, J=2.3 \mathrm{~Hz}, 1 \mathrm{H}), 4.95(\mathrm{~s}, 1 \mathrm{H}), 4.80-4.76(\mathrm{~m}$, $1 \mathrm{H}), 4.57(\mathrm{~s}, 1 \mathrm{H}), 4.50(\mathrm{td}, J=10.4,4.1 \mathrm{~Hz}, 1 \mathrm{H}), 4.39(\mathrm{~s}, 1 \mathrm{H}), 4.18(\mathrm{t}, J=4.4 \mathrm{~Hz}, 1 \mathrm{H}), 4.12(\mathrm{t}, J=4.7 \mathrm{~Hz}$, $2 \mathrm{H}), 4.06(\mathrm{t}, J=6.0 \mathrm{~Hz}, 1 \mathrm{H}), 4.01(\mathrm{q}, J=5.7 \mathrm{~Hz}, 1 \mathrm{H}), 3.95-3.88(\mathrm{~m}, 2 \mathrm{H}), 3.88(\mathrm{~d}, J=5.0 \mathrm{~Hz}, 1 \mathrm{H}), 3.85-$ $3.79(\mathrm{~m}, 1 \mathrm{H}), 3.76(\mathrm{~d}, J=4.2 \mathrm{~Hz}, 1 \mathrm{H}), 3.72-3.69(\mathrm{~m}, 2 \mathrm{H}), 3.62(\mathrm{t}, J=5.7 \mathrm{~Hz}, 1 \mathrm{H}), 3.60-3.53(\mathrm{~m}, 2 \mathrm{H})$, 3.51-3.44 (m, $1 \mathrm{H}), 3.36(\mathrm{t}, J=7.8 \mathrm{~Hz}, 1 \mathrm{H}), 3.34-3.30(\mathrm{~m}, 1 \mathrm{H}), 3.22(\mathrm{~s}, 3 \mathrm{H}), 3.22-3.19(\mathrm{~m}, 1 \mathrm{H}), 2.68(\mathrm{~d}$, $J=2.9 \mathrm{~Hz}, 1 \mathrm{H}), 2.59-2.44(\mathrm{~m}, 6 \mathrm{H}), 2.44-2.34(\mathrm{~m}, 2 \mathrm{H}), 2.34-2.22(\mathrm{~m}, 4 \mathrm{H}), 2.21-2.08(\mathrm{~m}, 7 \mathrm{H}), 2.07-1.99$ (m, 4H), 1.98-1.85 (m, 3 H), 1.84-1.68 (m, 5 H), 1.61-1.53 (m, $2 \mathrm{H}), 1.53-1.42(\mathrm{~m}, 7 \mathrm{H}), 1.36(\mathrm{t}, J=3.0 \mathrm{~Hz}$, $1 \mathrm{H}), 1.33(\mathrm{~d}, J=6.9 \mathrm{~Hz}, 3 \mathrm{H}), 1.09(\mathrm{~d}, J=6.9 \mathrm{~Hz}, 3 \mathrm{H}), 1.00(\mathrm{~d}, J=6.9 \mathrm{~Hz}, 3 \mathrm{H}), 0.95(\mathrm{~d}, J=6.4 \mathrm{~Hz}, 3 \mathrm{H}) \mathrm{ppm}$; ${ }^{13} \mathrm{C}$ NMR $\left(151 \mathrm{MHz}, \mathrm{C}_{6} \mathrm{D}_{6}\right): \delta 152.4,151.5,114.8,110.2,105.1,104.8,104.8,97.0,82.6,81.0,79.4,79.2$, $78.3,78.2,77.7,77.6,77.3,77.0,76.9,76.2,75.8,74.6,74.5,74.1,73.9,73.1,72.3,72.1,68.7,63.7,55.7$, $55.4,47.7,45.7,45.5,43.1,42.0,39.9,39.0,37.7,37.1,36.2,35.7,34.3,32.1,31.8,30.7,30.5,30.3,30.24$, 
$30.22,30.18,29.8,29.2,26.1,18.2,18.1,17.5,15.8$ ppm; HRMS (ESI-TOF) $m / z:[\mathrm{M}+\mathrm{Na}]^{+}$Calcd. for $\mathrm{C}_{60} \mathrm{H}_{86} \mathrm{O}_{19} \mathrm{Na}^{+}$1133.5656; Found 1133.5634.

One-Pot Procedure Toward $[(1 S, 2 S, 4 R, 7 S, 9 R, 10 S, 12 S, 14 R, 16 R)-12-\{2-[(2 S, 5 S)-5-\{2-[(2 S, 4 R, 6 R)-6-$ $\left\{\left[\left(2 R, 2^{\prime \prime} R, 3 \mathrm{a} R, 3 \mathrm{a}^{\prime} S, 3 \mathrm{a}^{\prime \prime} R, 4 \mathrm{a} S, 4 \mathrm{a}^{\prime \prime} S, 5 R, 5^{\prime} R, 5^{\prime \prime} S, 6 R, 7 S, 7^{\prime} S, 7 \mathrm{a}^{\prime} S, 8 \mathrm{a} S, 8 \mathrm{a}^{\prime \prime} S, 9 \mathrm{a} R, 9 \mathrm{a}^{\prime \prime} R\right)-6-\mathrm{Hydroxy-2"}-\right.\right.$ methoxy-5,5",7'-trimethylicosahydro-2" $\mathrm{H,3H,3}$ ' $\mathrm{H}$-dispiro[furo[3,2-b]pyrano[2,3-e]pyran-2,2'-furo[3,2-b]pyran-5',7"-furo[3,2-b]pyrano[2,3-e]pyran]-7-yl]methyl\}-4-methyl-5-methylidenetetrahydro2H-pyran-2-yl]ethyl\}-4-methylidenetetrahydrofuran-2-yl]ethyl\}-3,8,11,15,17-pentaoxapentacyclo-

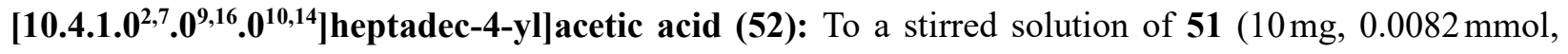
1.0 equiv) in toluene $(1.5 \mathrm{~mL})$ at $23^{\circ} \mathrm{C}$ was added $\mathrm{DBU}(0.13 \mathrm{~mL}, 0.82 \mathrm{mmol}, 100$ equiv). The resulting mixture was warmed to $110^{\circ} \mathrm{C}$ and stirred for $1 \mathrm{~h}$ before it was allowed to cool to $23^{\circ} \mathrm{C}$. Then, a $\mathrm{LiOH}$ solution in $\mathrm{THF} / \mathrm{H}_{2} \mathrm{O}\left[2 \mathrm{~mL}, 0.5 \mathrm{M}\right.$ in THF: $\left.\mathrm{H}_{2} \mathrm{O}(1: 1, v / v)\right]$ was added. The resulting mixture was stirred for $3.5 \mathrm{~h}$ before it was quenched by the addition of aq. $\mathrm{HCl}(0.3 \mathrm{M}, 10 \mathrm{~mL})$. The layers were separated, and the aqueous layer was extracted with $\mathrm{CH}_{2} \mathrm{Cl}_{2}(4 \times 10 \mathrm{~mL})$. The combined organic extracts were washed with aq. $\mathrm{HCl}(0.1 \mathrm{M}, 10 \mathrm{~mL})$, dried over $\mathrm{Na}_{2} \mathrm{SO}_{4}$, filtered, and concentrated under reduced pressure. Flash column chromatography $\left(\mathrm{SiO}_{2}\right.$, EtOAc:MeOH 100:1, v/v $\rightarrow$ 3:1, v/v) of the residue afforded carboxylic acid 52 (6.0 mg, $0.0055 \mathrm{mmol}, 67 \%$ yield) as a colorless oil. 
$[1 " R-[1 " S, 2$ " $R, 3$ "a $R, 3$ "b $S, 5$ " $S, 8$ " $S, 11$ " $S, 14$ " $S, 16$ " $R, 18$ " $R, 19$ "a $S, 20$ "a $S, 21$ "a $R, 23$ " $R[3$ 'a $S, 5$ ' $R(2 S, 3 \mathrm{a} R$, 4a $\left.S, 5 S, 8 \mathrm{a} S, 9 \mathrm{a} R), 7^{\prime} S, 7^{\prime} \mathrm{a} S\right], 24$ " a $R, 25$ "a $S, 26 " S, 26$ "a $R, 30$ " $R, 31$ "a $S, 32$ " $S, 33$ " a $R$ ]l-dotetracontahydro-2methoxy-5,7',16",26"-tetramethyl-10",17"-bis(methylene)-dispiro[furo[3,2-b]pyrano[2,3-e]pyran$7(2 H), 5^{\prime}-[5 H]$ furo[3,2-b]pyran-2' $\left(3^{\prime} H\right), 23^{\prime \prime}\left(6^{\prime \prime} H\right)-[1,5: 8,11: 14,18]$ triepoxy[30,32]ethano[2,5]methano$[2 H, 5 H, 28 H]$ furo $\left[2^{\prime}, 3^{\prime}: 5,6\right]$ pyrano $[4,3-b]$ furo $\left[2^{\prime \prime}, 3^{\prime \prime}: 5^{\prime}, 6^{\prime}\right]$ pyrano $\left[2^{\prime}, 3^{\prime}: 5,6\right]$ pyrano $[3,2-i][1,4,8]$ trioxacyclopentacosin]-28"-one (53): To a stirred solution of 2-methyl-6-nitrobenzoic anhydride (8.8 mg,

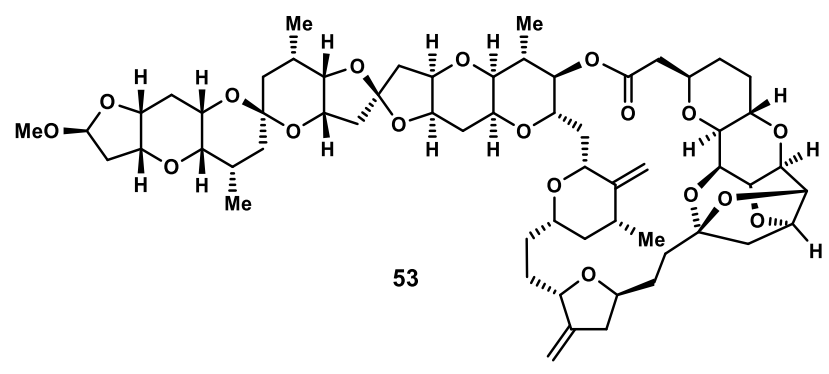
$0.026 \mathrm{mmol}, 5.0$ equiv.) and $N, N$-dimethylpyridin4-amine $\quad(6.2 \mathrm{mg}, \quad 0.051 \mathrm{mmol}, \quad 10.0$ equiv $)$ in toluene $(1.5 \mathrm{~mL})$ at $70^{\circ} \mathrm{C}$ was added a solution of 52 (5.7 mg, $0.0051 \mathrm{mmol}, 1.0$ equiv) and $N$-ethyl- $N$ (propan-2-yl)propan-2-amine $(5.0 \mu \mathrm{L}, 0.026 \mathrm{mmol}$,

5.0 equiv.) in toluene $(1 \mathrm{~mL})$ via a syringe pump over $12 \mathrm{~h}$. After completion of the addition, the syringe was rinsed with toluene $(0.3 \mathrm{~mL})$. After being stirred for additional $0.5 \mathrm{~h}$, the reaction mixture was allowed to cool to $23{ }^{\circ} \mathrm{C}$ and concentrated under reduced pressure. Flash column chromatography $\left(\mathrm{SiO}_{2}\right.$, hexanes/ EtOAc $2: 1, v / v \rightarrow 1: 2, v / v)$ of the residue afforded macrolide $53(3.9 \mathrm{mg}, 0.0036 \mathrm{mmol}, 70 \%$ yield $)$ as a colorless oil. 53: $\mathrm{R}_{\mathrm{f}}=0.80\left(\mathrm{SiO}_{2}, 100 \%\right.$ EtOA$) ;[\alpha]_{\mathrm{D}}^{23}=-35.0\left(c=0.10\right.$, EtOAc); FTIR (film): $v_{\max } 2925$, 2853, 1735, 1263, 1187, 1076, 1023, 898, 798, $731 \mathrm{~cm}^{-1} ;{ }^{1} \mathrm{H}$ NMR $\left(600 \mathrm{MHz}, \mathrm{C}_{6} \mathrm{D}_{6}\right): \delta 5.15$ (dd, $J=5.8$, $3.7 \mathrm{~Hz}, 1 \mathrm{H}), 5.01(\mathrm{~d}, J=2.5 \mathrm{~Hz}, 1 \mathrm{H}), 4.96(\mathrm{~s}, 2 \mathrm{H}), 4.93(\mathrm{dd}, J=10.6,6.4 \mathrm{~Hz}, 1 \mathrm{H}), 4.84(\mathrm{~d}, J=1.8 \mathrm{~Hz}, 1 \mathrm{H})$, 4.65-4.56 (m, 2 H), 4.55-4.48 (m, 1 H), 4.30-4.27 (m, $1 \mathrm{H}), 4.15$ (t, J=4.6 Hz, 1 H), 4.10-4.03 (m, 2H), $3.99(\mathrm{dt}, J=12.1,6.2 \mathrm{~Hz}, 1 \mathrm{H}), 3.95-3.86(\mathrm{~m}, 3 \mathrm{H}), 3.84-3.79(\mathrm{~m}, 3 \mathrm{H}), 3.80-3.72(\mathrm{~m}, 2 \mathrm{H}), 3.63$ (dd, $J=6.6$, $4.1 \mathrm{~Hz}, 1 \mathrm{H}), 3.58(\mathrm{dd}, J=5.5,2.4 \mathrm{~Hz}, 1 \mathrm{H}), 3.42(\mathrm{dt}, J=11.7,6.0 \mathrm{~Hz}, 1 \mathrm{H}), 3.33(\mathrm{~d}, J=4.5 \mathrm{~Hz}, 1 \mathrm{H}), 3.21(\mathrm{~s}$, $3 \mathrm{H}), 2.73-2.67(\mathrm{~m}, 3 \mathrm{H}), 2.66-2.61(\mathrm{~m}, 2 \mathrm{H}), 2.58(\mathrm{dd}, J=12.8,5.8 \mathrm{~Hz}, 2 \mathrm{H}), 2.46(\mathrm{dd}, J=14.1,4.8 \mathrm{~Hz}, 1 \mathrm{H})$, 2.39-2.27 (m, 7H), 2.26-2.21 (m, 3 H), 2.19-2.10 (m, 6H), 2.05 (ddd, $J=14.1,5.7,3.7 \mathrm{~Hz}, 2 \mathrm{H}), 2.01-1.91$ $(\mathrm{m}, 2 \mathrm{H}), 1.87(\mathrm{td}, J=9.0,4.4 \mathrm{~Hz}, 1 \mathrm{H}), 1.73-1.65(\mathrm{~m}, 2 \mathrm{H}), 1.63-1.56(\mathrm{~m}, 1 \mathrm{H}), 1.54-1.48(\mathrm{~m}, 2 \mathrm{H}), 1.48-$ $1.42(\mathrm{~m}, 5 \mathrm{H}), 1.32-1.29(\mathrm{~m}, 4 \mathrm{H}), 1.16(\mathrm{~d}, J=6.6 \mathrm{~Hz}, 3 \mathrm{H}), 1.05(\mathrm{~d}, J=6.4 \mathrm{~Hz}, 3 \mathrm{H}), 1.02(\mathrm{~d}, J=6.9 \mathrm{~Hz}, 3 \mathrm{H})$, $0.98(\mathrm{~d}, J=7.0 \mathrm{~Hz}, 3 \mathrm{H}) . \mathrm{ppm} ;{ }^{13} \mathrm{C} \mathrm{NMR}\left(151 \mathrm{MHz}, \mathrm{C}_{6} \mathrm{D}_{6}\right): \delta 171.1,152.9,152.5,114.3,110.2,104.9,104.4$, 104.0, 97.0, 82.5, 81.0, 79.0, 78.5, 78.4, 78.2, 77.4, 77.33, 77.31, 76.9, 75.4, 75.3, 75.1, 75.0, 74.2, 74.1, 
$73.9,73.8,72.4,72.3,71.9,68.4,67.3,63.8,55.4,48.7,45.3,44.8,44.0,42.1,40.6,39.2,37.7,37.5,37.2$ $36.7,36.5,35.1,32.8,31.0,30.9,30.6,30.3,29.6,29.2,28.7,25.9,18.2,18.0,17.6,15.1$ ppm; HRMS (ESITOF) $m / z$ : $[\mathrm{M}+\mathrm{Na}]^{+}$Calcd. for $\mathrm{C}_{60} \mathrm{H}_{84} \mathrm{O}_{18} \mathrm{Na}^{+}$1115.5550; Found 1115.5531

$\left(1^{\prime \prime} S, 2 R, 2^{\prime} R, 2^{\prime \prime} R, 3\right.$ 'a $S, 3$ "a $R, 3$ "b $S, 4 S, 4 \mathrm{a} S, 5^{\prime \prime} S, 6 R, 7 R, 7 ' S, 7^{\prime} \mathrm{a} S, 8^{\prime \prime} S, 8 \mathrm{a} S, 11^{\prime \prime} S, 14^{\prime \prime} S, 16^{\prime \prime} R, 18^{\prime \prime} R, 19$ "a $S$, 20 "a $S, 21^{\prime \prime} \mathrm{a} R, 24$ " a $R, 25$ "a $S, 26$ " $S, 26$ "a $R, 30 " R, 31$ "a $S, 32$ " $S, 33$ "a $R$ )-Tetracontahydro-7-hydroxy-4,7", 16",26"-tetramethyl-10",17"-bis(methylene)-28"-oxo-dispiro[pyrano[3,2-b]pyran-2(3H),5'-[5H] furo[3,2-b]pyran-2'(3'H),23"(6" $H)-[1,5: 8,11: 14,18]$ triepoxy[30,32] ethano[2,5] methano[2H,5H,28H] furo $\left[2^{\prime}, 3^{\prime}: 5,6\right]$ pyrano $[4,3-b]$ furo $\left[2^{\prime \prime}, 3^{\prime \prime}: 5^{\prime}, 6^{\prime}\right]$ pyrano $\left[2^{\prime}, 3^{\prime}: 5,6\right]$ pyrano $[3,2-i][1,4,8]$ trioxacyclopentacosin]-6-acetic acid (2): A solution of $\mathbf{5 3}\left(2.7 \mathrm{mg}, 0.0025 \mathrm{mmol}, 1.0\right.$ equiv) in $\mathrm{AcOH} / \mathrm{H}_{2} \mathrm{O}(0.6 \mathrm{~mL}$,

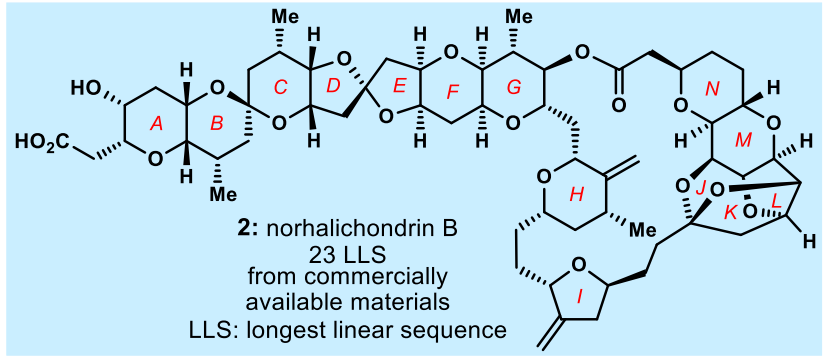
$2: 1, v / v)$ at $50^{\circ} \mathrm{C}$ was stirred $1 \mathrm{~h}$ before it was concentrated under reduced pressure to the give the crude corresponding hemiacetal and its tautomer.

To a stirred solution of the above obtained hemiacetal and tautomer in $t$-BuOH/THF $(1.0 \mathrm{~mL}, 4: 1, v / v)$ at $23^{\circ} \mathrm{C}$ was added 2-methylbut-2-ene $(13 \mu \mathrm{L}$, $0.25 \mathrm{mmol}$, 25.0 equiv), followed by a solution of $\mathrm{NaClO}_{2}(2.2 \mathrm{mg}, 0.025 \mathrm{mmol}, 10.0$ equiv) and $\mathrm{NaH}_{2} \mathrm{PO}_{4} \cdot \mathrm{H}_{2} \mathrm{O}\left(8.0 \mathrm{mg}, 4.5 \mathrm{mmol}, 20\right.$ equiv) in $\mathrm{H}_{2} \mathrm{O}(0.15 \mathrm{~mL})$. The resulting mixture was stirred for $0.5 \mathrm{~h}$ before it was diluted with aq. $\mathrm{HCl}(0.05 \mathrm{M}, 3 \mathrm{~mL})$. The layers were separated, and the aqueous layer was extracted with $\mathrm{CH}_{2} \mathrm{Cl}_{2}(4 \times 10 \mathrm{~mL})$. The combined organic extracts were dried over $\mathrm{Na}_{2} \mathrm{SO}_{4}$, filtered, and concentrated under reduced pressure. Flash column chromatography $\left(\mathrm{SiO}_{2}, \mathrm{EtOAc:MeOH} \mathrm{50:1,} \mathrm{v/v \rightarrow} \mathrm{4:1,}\right.$ $v / v)$ of the residue afforded norhalichondrin B $(2 ; 1.8 \mathrm{mg}, 0.0017 \mathrm{mmol}, 68 \%$ for the two steps $)$ as a colorless oil. 2: $\mathrm{R}_{\mathrm{f}}=0.50\left(\mathrm{SiO}_{2}, \mathrm{EtOAc} / \mathrm{MeOH} 5: 1, v / v\right) ;[\alpha]_{\mathrm{D}}^{23}=-55.4(c=0.10, \mathrm{MeOH})$; FTIR (film): $v_{\max }$ 3446, 2928, 2872, 1735, 1649, 1572, 1407, 1338, 1268, 1190, 1134, 1071, 1045, 1019, $995 \mathrm{~cm}^{-1}$; ${ }^{1} \mathrm{H}$ NMR $\left(600 \mathrm{MHz}, \mathrm{CD}_{3} \mathrm{OD}\right): \delta 5.06(\mathrm{~d}, J=1.5 \mathrm{~Hz}, 1 \mathrm{H}), 5.02$ (brs, $\left.1 \mathrm{H}\right), 4.88(\mathrm{~s}, 1 \mathrm{H}$, from HSQC), $4.81(\mathrm{~s}, 1 \mathrm{H}), 4.70$ $(\mathrm{t}, J=4.6 \mathrm{~Hz}, 1 \mathrm{H}), 4.64-4.57(\mathrm{~m}, 2 \mathrm{H}), 4.45(\mathrm{~d}, J=11.0 \mathrm{~Hz}, 1 \mathrm{H}), 4.33(\mathrm{dt}, J=10.4,5.1 \mathrm{~Hz}, 1 \mathrm{H}), 4.31-4.28$ (m, $1 \mathrm{H}), 4.27-4.21(\mathrm{~m}, 1 \mathrm{H}), 4.18(\mathrm{dd}, J=6.6,4.5 \mathrm{~Hz}, 1 \mathrm{H}), 4.13-4.09(\mathrm{~m}, 3 \mathrm{H}), 4.10-4.04(\mathrm{~m}, 1 \mathrm{H}), 3.98(\mathrm{~d}$, 
$J=2.6 \mathrm{~Hz}, 1 \mathrm{H}), 3.92-3.84(\mathrm{~m}, 2 \mathrm{H}), 3.82-3.75(\mathrm{~m}, 2 \mathrm{H}), 3.74-3.67(\mathrm{~m}, 2 \mathrm{H}), 3.63-3.58(\mathrm{~m}, 2 \mathrm{H}), 3.31$ (m, $1 \mathrm{H}$, from HSQC), $3.21(\mathrm{dd}, J=6.5,4.6 \mathrm{~Hz}, 1 \mathrm{H}), 2.98(\mathrm{dd}, J=9.5,2.1 \mathrm{~Hz}, 1 \mathrm{H}), 2.83-2.77(\mathrm{~m}, 1 \mathrm{H}), 2.56(\mathrm{dd}$, $J=17.5,9.7 \mathrm{~Hz}, 1 \mathrm{H}), 2.48(\mathrm{~d}, J=6.7 \mathrm{~Hz}, 2 \mathrm{H}), 2.45(\mathrm{dd}, J=17.8,2.3 \mathrm{~Hz}, 1 \mathrm{H}), 2.39(\mathrm{dd}, J=13.2,5.8 \mathrm{~Hz}, 1 \mathrm{H})$, $2.33(\mathrm{~d}, J=2.9 \mathrm{~Hz}, 2 \mathrm{H}), 2.31-2.23(\mathrm{~m}, 4 \mathrm{H}), 2.22-2.13(\mathrm{~m}, 3 \mathrm{H}), 2.11-2.03(\mathrm{~m}, 5 \mathrm{H}), 2.03-1.92(\mathrm{~m}, 4 \mathrm{H})$, $1.87-1.79(\mathrm{~m}, 2 \mathrm{H}), 1.76-1.66(\mathrm{~m}, 3 \mathrm{H}), 1.60(\mathrm{td}, J=12.7,6.2 \mathrm{~Hz}, 1 \mathrm{H}), 1.55-1.47$ (m, $3 \mathrm{H}), 1.46-1.28(\mathrm{~m}$, $6 \mathrm{H}), 1.10(\mathrm{~d}, J=6.5 \mathrm{~Hz}, 3 \mathrm{H}), 1.06(\mathrm{~d}, J=7.1 \mathrm{~Hz}, 3 \mathrm{H}), 1.02(\mathrm{~d}, J=11.8 \mathrm{~Hz}, 1 \mathrm{H}), 0.98(\mathrm{~d}, J=7.4 \mathrm{~Hz}, 3 \mathrm{H})$, $0.96(\mathrm{~d}, J=7.3 \mathrm{~Hz}, 3 \mathrm{H}) \mathrm{ppm} ;{ }^{13} \mathrm{C} \mathrm{NMR}\left(151 \mathrm{MHz}, \mathrm{CD}_{3} \mathrm{OD}\right): \delta 179.8,172.8,153.3,153.2,114.8,111.3$, $105.7,104.8,98.5,83.8,82.4,80.7,79.10,79.08,78.98,77.95,77.9,77.32,77.30,77.2,76.3,76.1,75.8$, 75.3, 75.0, 74.9, 73.8, 72.6, 69.6, 68.2, 68.1, 65.6, 45.5, 44.9, 41.5, 41.2, 39.7, 38.3, 38.1, 37.8, 37.4, 37.2, 35.8, 35.7, 33.0, 31.8, 31.3, 31.1, 30.9, 30.1, 29.4, 27.3, 18.4, 18.1, 17.4, 15.8 ppm; HRMS (ESI-TOF) $m / z$ : $[\mathrm{M}+\mathrm{Na}]^{+}$Calcd. for $\mathrm{C}_{59} \mathrm{H}_{82} \mathrm{O}_{19} \mathrm{Na}^{+}$1117.5343; Found 1117.5330. 
Table 1: Comparison of ${ }^{1} \mathrm{H}$ NMR (600 MHz, $\left.\mathrm{CD}_{3} \mathrm{OD}\right)$ Data for Norhalichondrin B (1). ${ }^{7,8,9}$

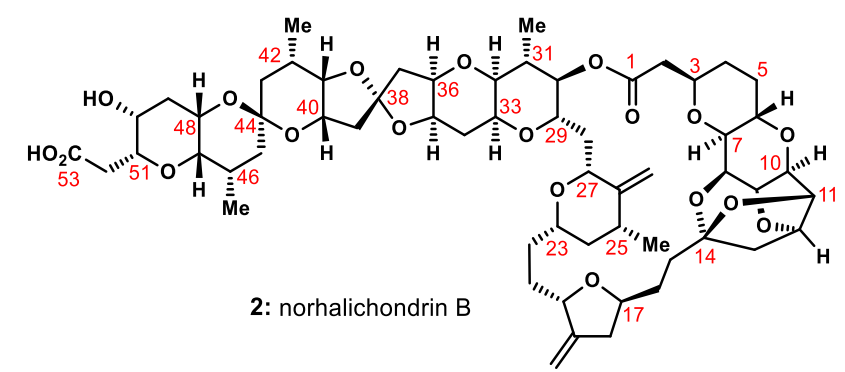

\begin{tabular}{|l|c|c|c|}
\hline Entry & $\begin{array}{c}\text { Synthetic 2 } \\
\text { (current } \\
\text { work) }\end{array}$ & Natural 2 & $\begin{array}{c}\text { Synthetic 2 } \\
\text { (Kishi's } \\
\text { work) }\end{array}$ \\
\hline $\mathrm{H} 2$ & 2.45 & 2.44 & 2.45 \\
\hline $\mathrm{H} 2$ & 2.56 & 2.56 & 2.56 \\
\hline $\mathrm{H} 3$ & 3.88 & 3.88 & 3.88 \\
\hline $\mathrm{H} 6$ & 4.33 & 4.33 & 4.32 \\
\hline $\mathrm{H} 7$ & 2.98 & 2.97 & 2.98 \\
\hline $\mathrm{H} 8$ & 4.30 & 4.30 & 4.30 \\
\hline $\mathrm{H} 9$ & 4.10 & 4.10 & 4.10 \\
\hline $\mathrm{H} 10$ & 4.18 & 4.18 & 4.18 \\
\hline $\mathrm{H} 11$ & 4.60 & 4.60 & 4.60 \\
\hline $\mathrm{H} 12$ & 4.70 & 4.70 & 4.70 \\
\hline $\mathrm{H} 13$ & 2.00 & 2.00 & 2.00 \\
\hline $\mathrm{H} 13$ & 2.08 & 2.08 & 2.08 \\
\hline $\mathrm{H} 17$ & 4.08 & 4.08 & 4.08 \\
\hline $\mathrm{H} 18$ & 2.33 & 2.32 & 2.32 \\
\hline $\mathrm{H} 18$ & 2.81 & 2.80 & 2.81 \\
\hline $\mathrm{C} 19 \mathrm{CH}_{2}$ & 5.02 & 5.01 & 5.01 \\
\hline $\mathrm{C} 19 \mathrm{CH}_{2}$ & 5.06 & 5.06 & 5.06 \\
\hline $\mathrm{H} 20$ & 4.45 & 4.45 & 4.45 \\
\hline $\mathrm{H} 23$ & 3.71 & 3.71 & 3.71 \\
\hline $\mathrm{C} 25-\mathrm{Me}_{2}$ & 1.10 & 1.09 & 1.10 \\
\hline $\mathrm{C} 26 \mathrm{CH}_{2}$ & 4.81 & 4.82 & 4.81 \\
\hline $\mathrm{C} 26 \mathrm{CH}_{2}$ & 4.88 & 4.87 & 4.88 \\
\hline
\end{tabular}

\begin{tabular}{|l|c|c|c|}
\hline Entry & $\begin{array}{c}\text { Synthetic 2 } \\
\text { (current } \\
\text { work) }\end{array}$ & Natural 2 & $\begin{array}{c}\text { Synthetic 2 } \\
\text { (Kishi's } \\
\text { work) }\end{array}$ \\
\hline H27 & 3.61 & 3.61 & 3.61 \\
\hline H29 & 4.24 & 4.24 & 4.24 \\
\hline H30 & 4.62 & 4.61 & 4.63 \\
\hline C31-Me & 1.06 & 1.05 & 1.06 \\
\hline H32 & 3.21 & 3.21 & 3.22 \\
\hline H33 & 3.87 & 3.88 & 3.88 \\
\hline H35 & 4.10 & 4.10 & 4.10 \\
\hline H36 & 4.10 & 4.10 & 4.10 \\
\hline H40 & 3.98 & 3.98 & 3.99 \\
\hline H41 & 3.69 & 3.69 & 3.69 \\
\hline C42-Me & 0.98 & 0.98 & 0.98 \\
\hline C46-Me & 0.96 & 0.95 & 0.95 \\
\hline H47 & 3.31 & 3.30 & 3.30 \\
\hline H48 & 3.78 & 3.78 & 3.80 \\
\hline H49 & 1.95 & 1.95 & 1.95 \\
\hline H49 & 2.10 & 2.11 & 2.11 \\
\hline H50 & 3.61 & 3.61 & 3.61 \\
\hline H51 & 3.79 & 3.78 & 3.81 \\
\hline H52 & 2.48 & 2.47 & 2.60 \\
\hline H52 & 2.48 & 2.47 & 2.60 \\
\hline & & & \\
\hline & & & \\
\hline
\end{tabular}


Table 2: Comparison of ${ }^{13} \mathrm{C}$ NMR $\left(600 \mathrm{MHz}, \mathrm{CD}_{3} \mathrm{OD}\right)$ Data of Norhalichondrin B (1).$^{7,8,9}$

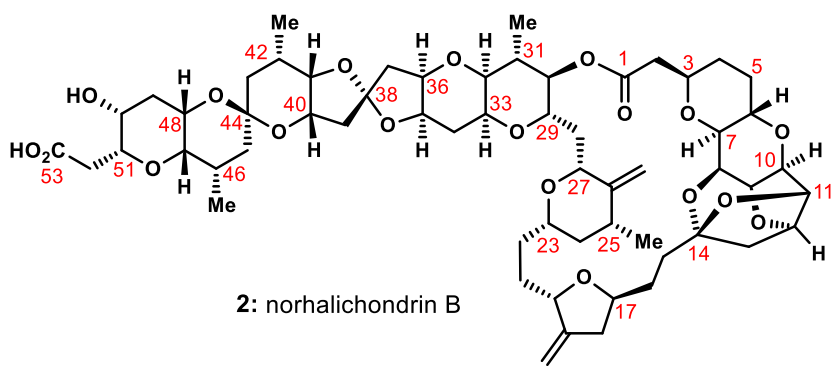

\begin{tabular}{|l|c|c|c|}
\hline Entry & $\begin{array}{c}\text { Synthetic 2 } \\
\text { (current } \\
\text { work) }\end{array}$ & $\begin{array}{c}\text { Natural } \\
\mathbf{2}\end{array}$ & $\begin{array}{c}\text { Synthetic 2 } \\
\text { (Kishi's } \\
\text { work) }\end{array}$ \\
\hline C1 & 172.8 & 173.6 & 172.8 \\
\hline C2 & 41.2 & 41.2 & 41.2 \\
\hline C3 & 74.9 & 75.0 & 75.0 \\
\hline C6 & 69.6 & 69.6 & 69.6 \\
\hline C7 & 79.1 & 79.1 & 79.0 \\
\hline C8 & 75.8 & 75.8 & 75.8 \\
\hline C9 & 74.9 & 74.9 & 74.9 \\
\hline C10 & 77.9 & 77.9 & 77.9 \\
\hline C11 & 83.8 & 83.8 & 83.4 \\
\hline C12 & 82.4 & 82.4 & 82.4 \\
\hline C13 & 49.6 & 49.4 & 49.4 \\
\hline C14 & 111.3 & 111.2 & 111.2 \\
\hline C17 & 76.3 & 76.3 & 76.3 \\
\hline C18 & 39.7 & 39.7 & 39.8 \\
\hline C19 & 153.2 & 154.0 & 153.3 \\
\hline C19= CH 2 & 105.7 & 105.7 & 105.6 \\
\hline C20 & 76.1 & 76.1 & 76.1 \\
\hline C23 & 75.3 & 75.3 & 75.4 \\
\hline C25 & 37.2 & 37.1 & 37.2 \\
\hline C25-Me & 18.4 & 18.4 & 18.4 \\
\hline C26 & 153.3 & 154.0 & 153.3 \\
\hline C26=CH2 2 & 104.8 & 104.8 & 104.8 \\
\hline C27 & 75.0 & 75.0 & 75.0 \\
\hline C29 & 73.8 & 73.8 & 73.8 \\
\hline
\end{tabular}

\begin{tabular}{|l|c|c|c|}
\hline Entry & $\begin{array}{c}\text { Synthetic 2 } \\
\text { (current } \\
\text { work) }\end{array}$ & $\begin{array}{c}\text { Natural } \\
\mathbf{2}\end{array}$ & $\begin{array}{c}\text { Synthetic 2 } \\
\text { (Kishi's } \\
\text { work) }\end{array}$ \\
\hline C30 & 77.3 & 77.3 & 77.4 \\
\hline C31 & 37.4 & 37.4 & 37.5 \\
\hline C31-Me & 15.8 & 15.8 & 15.8 \\
\hline C32 & 77.9 & 78.0 & 78.9 \\
\hline C33 & 65.6 & 65.6 & 65.6 \\
\hline C35 & 77.3 & 77.3 & 77.2 \\
\hline C36 & 78.0 & 78.0 & 78.1 \\
\hline C37 & 45.5 & 45.5 & 45.4 \\
\hline C38 & 114.8 & 114.8 & 114.7 \\
\hline C39 & 44.9 & 44.9 & 44.9 \\
\hline C40 & 72.6 & 72.6 & 72.7 \\
\hline C41 & 80.7 & 80.6 & 80.6 \\
\hline C42 & 27.3 & 27.3 & 27.3 \\
\hline C42-Me & 18.1 & 18.1 & 18.1 \\
\hline C44 & 98.5 & 98.5 & 98.5 \\
\hline C46 & 30.1 & 30.1 & 30.1 \\
\hline C46-Me & 17.4 & 17.4 & 17.3 \\
\hline C47 & 77.2 & 77.2 & 77.2 \\
\hline C48 & 68.2 & 68.2 & 68.2 \\
\hline C50 & 68.1 & 68.1 & 68.0 \\
\hline C51 & 79.1 & 79.1 & 79.1 \\
\hline C53 & 179.8 & 173.6 & 172.8 \\
\hline Note: The chemical shift for C53 in the current \\
\hline work was confirmed by HMBC. & \\
\hline
\end{tabular}




\section{III. ${ }^{1} \mathrm{H},{ }^{13} \mathrm{C}\left\{{ }^{1} \mathrm{H}\right\}$, COSY, HSQC, HMBC, and nOe NMR Spectra of Compounds}

吕

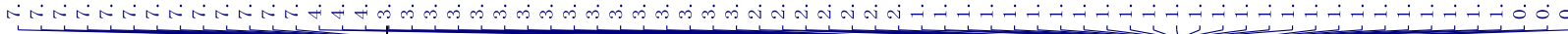

${ }^{1} \mathrm{H}$ NMR (600 MHz, $\mathrm{CDCl}_{3}$ ) of $\mathbf{1 4}$
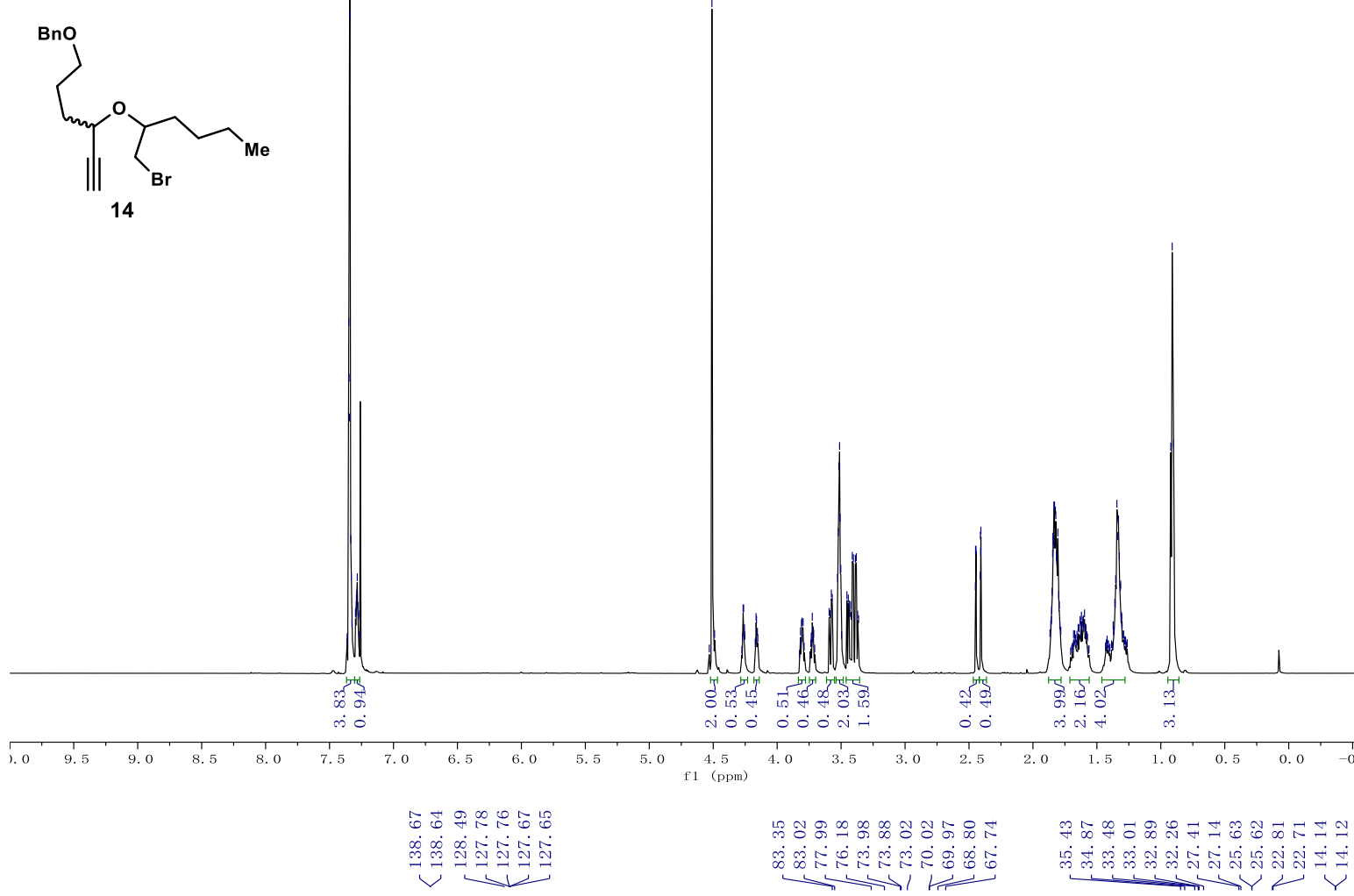

${ }^{13} \mathrm{C}$ NMR $\left(151 \mathrm{MHz}, \mathrm{CDCl}_{3}\right)$ of $\mathbf{1 4}$

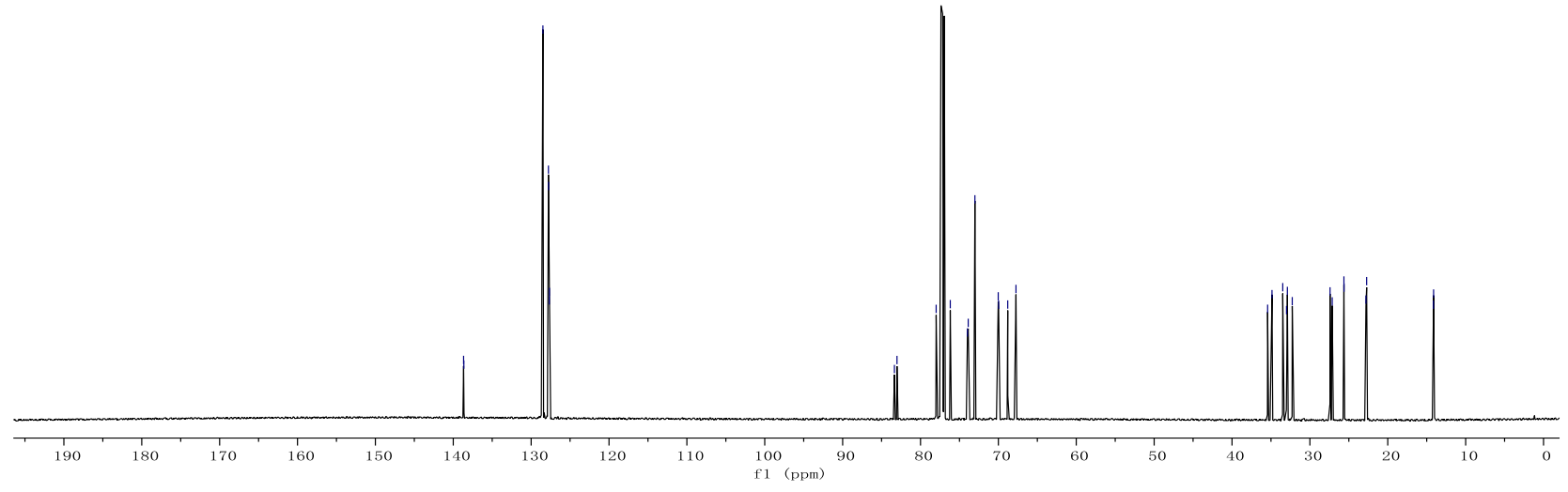




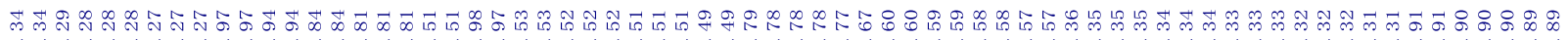

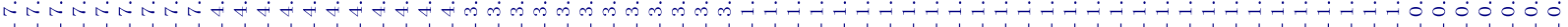

${ }^{1} \mathrm{H}$ NMR $\left(600 \mathrm{MHz}, \mathrm{CDCl}_{3}\right)$ of 15

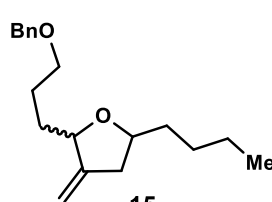

15
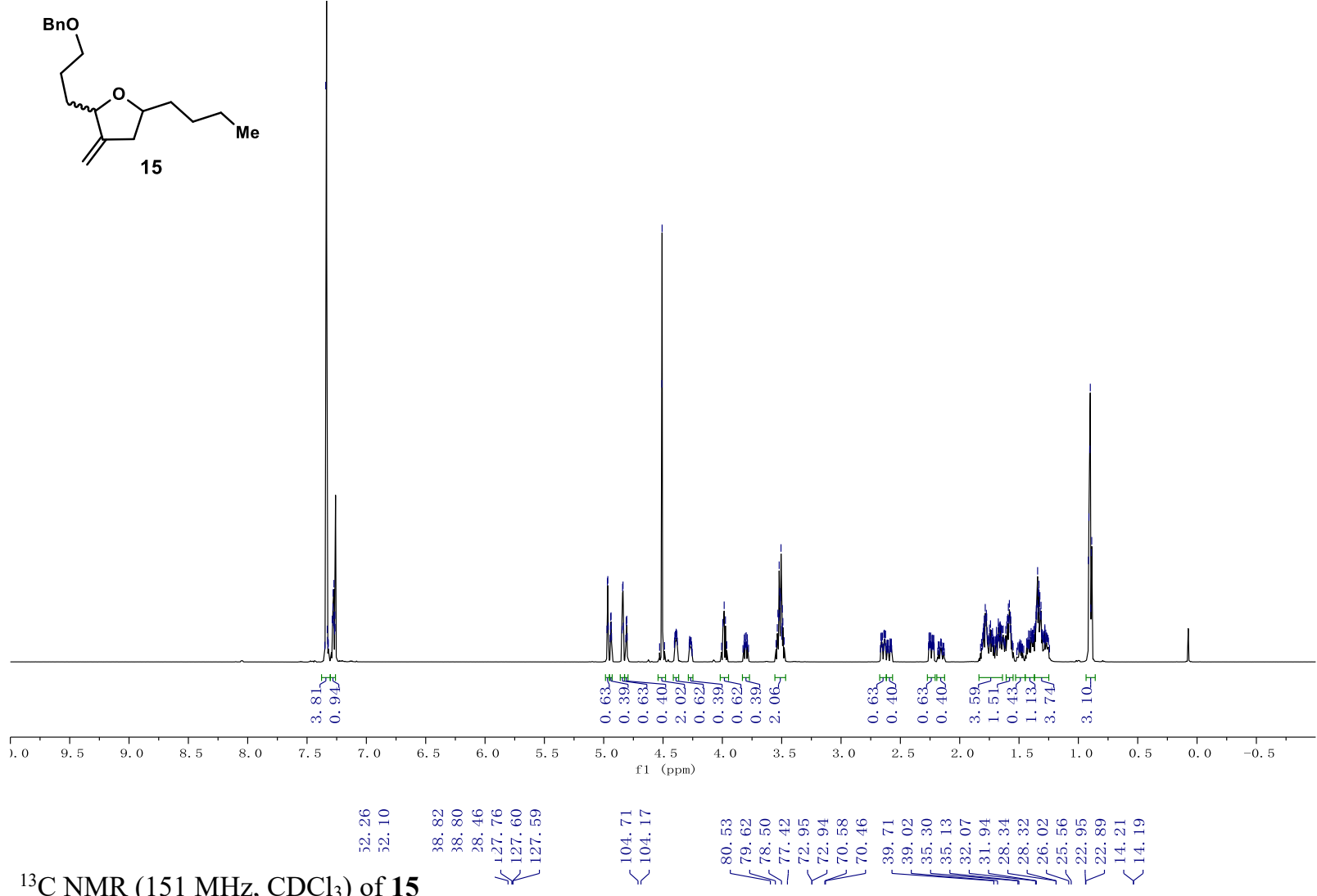

${ }^{13} \mathrm{C}$ NMR $\left(151 \mathrm{MHz}, \mathrm{CDCl}_{3}\right)$ of $\mathbf{1 5}$

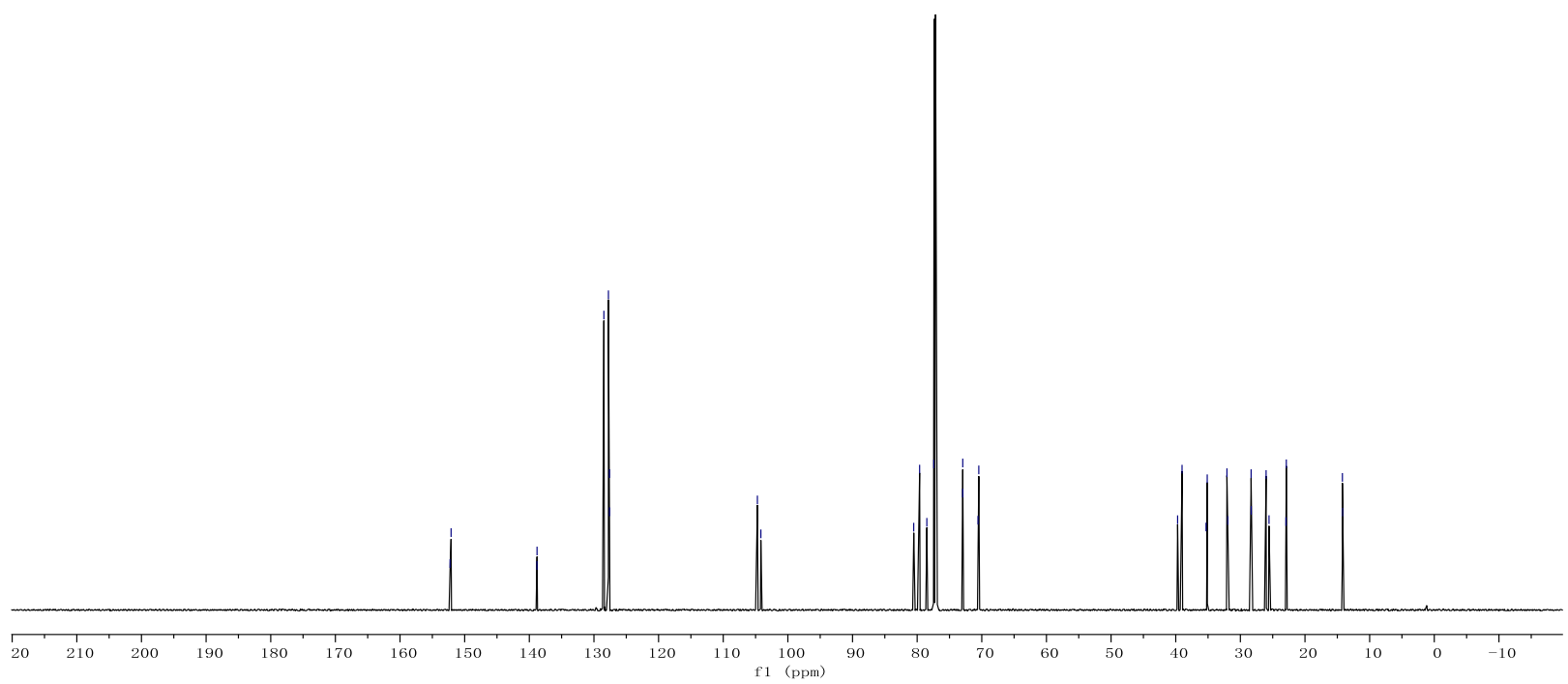




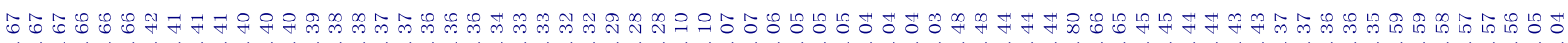

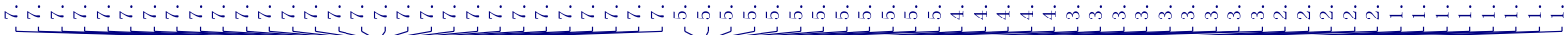

${ }^{1} \mathrm{H}$ NMR $\left(600 \mathrm{MHz}, \mathrm{CDCl}_{3}\right)$ of $\mathbf{1 8 b}$

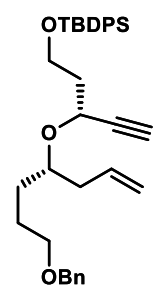

$18 \mathrm{~b}$

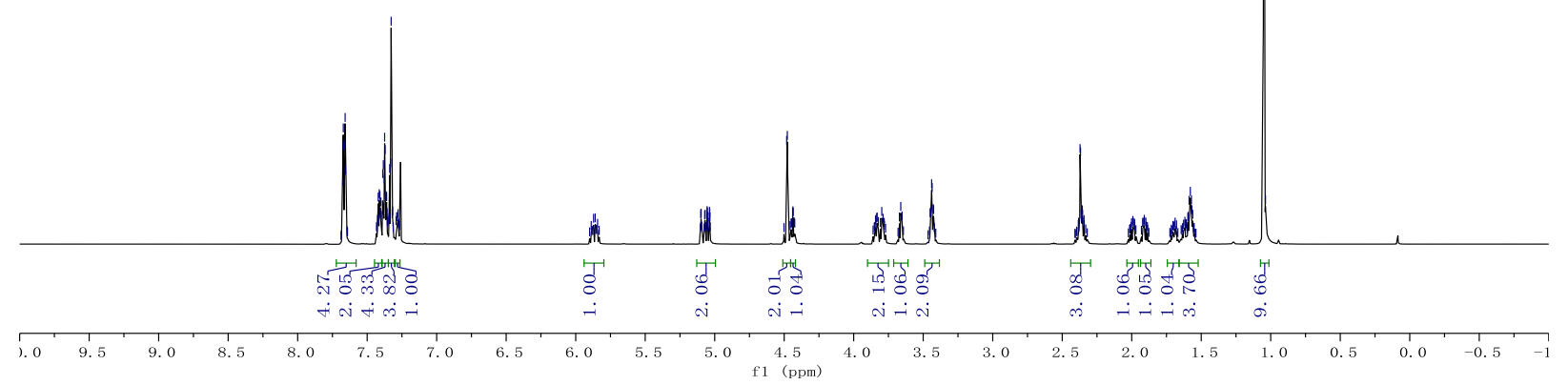

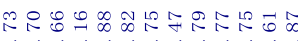

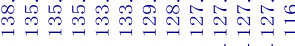

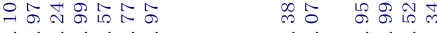

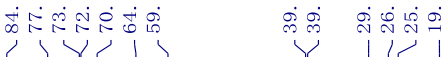

${ }^{13} \mathrm{C} \mathrm{NMR}\left(151 \mathrm{MHz}, \mathrm{CDCl}_{3}\right)$ of $\mathbf{1 8 b}$

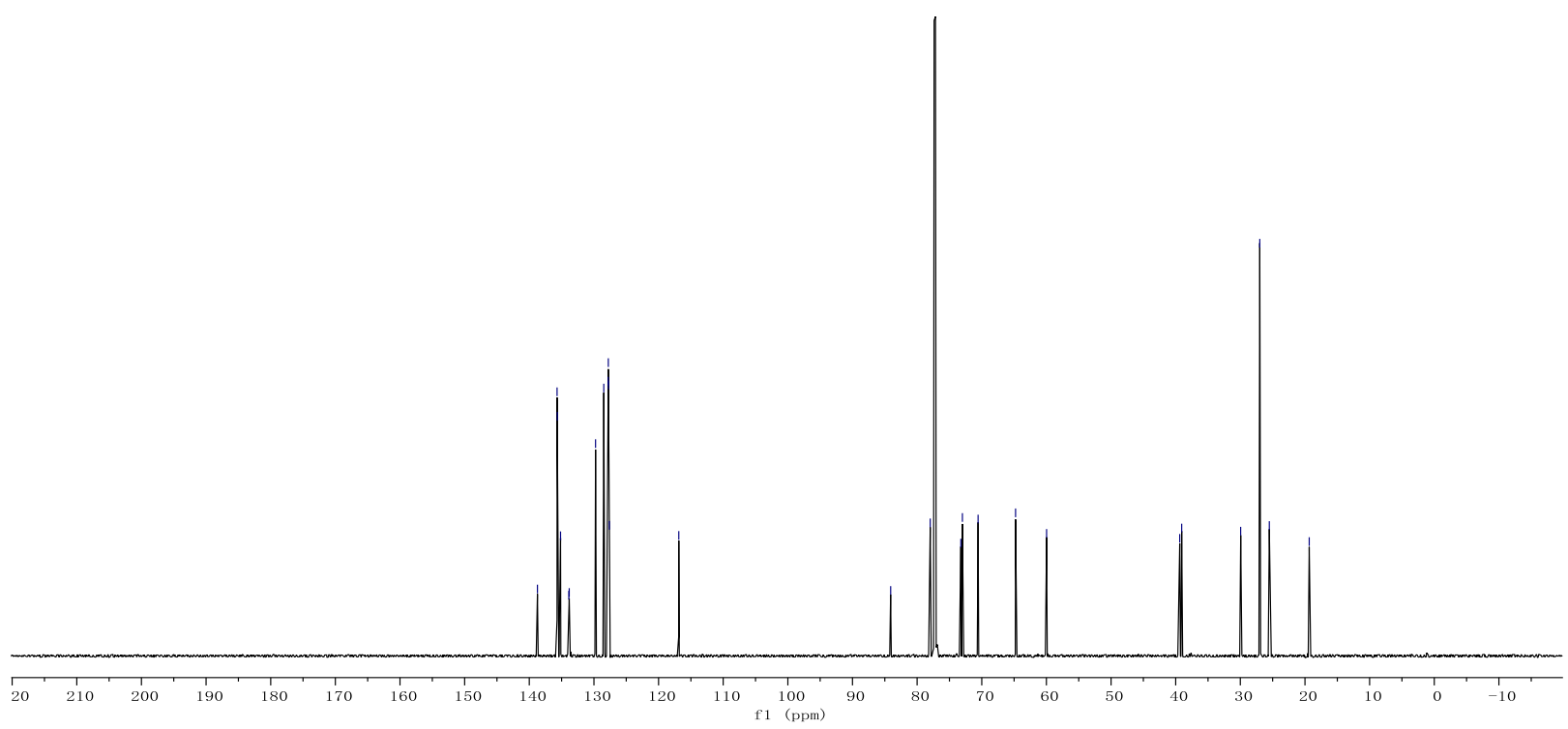


${ }^{1} \mathrm{H}$ NMR $\left(600 \mathrm{MHz}, \mathrm{CDCl}_{3}\right)$ of $\mathbf{1 8 a}$

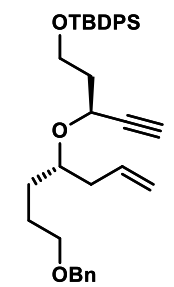

$18 a$
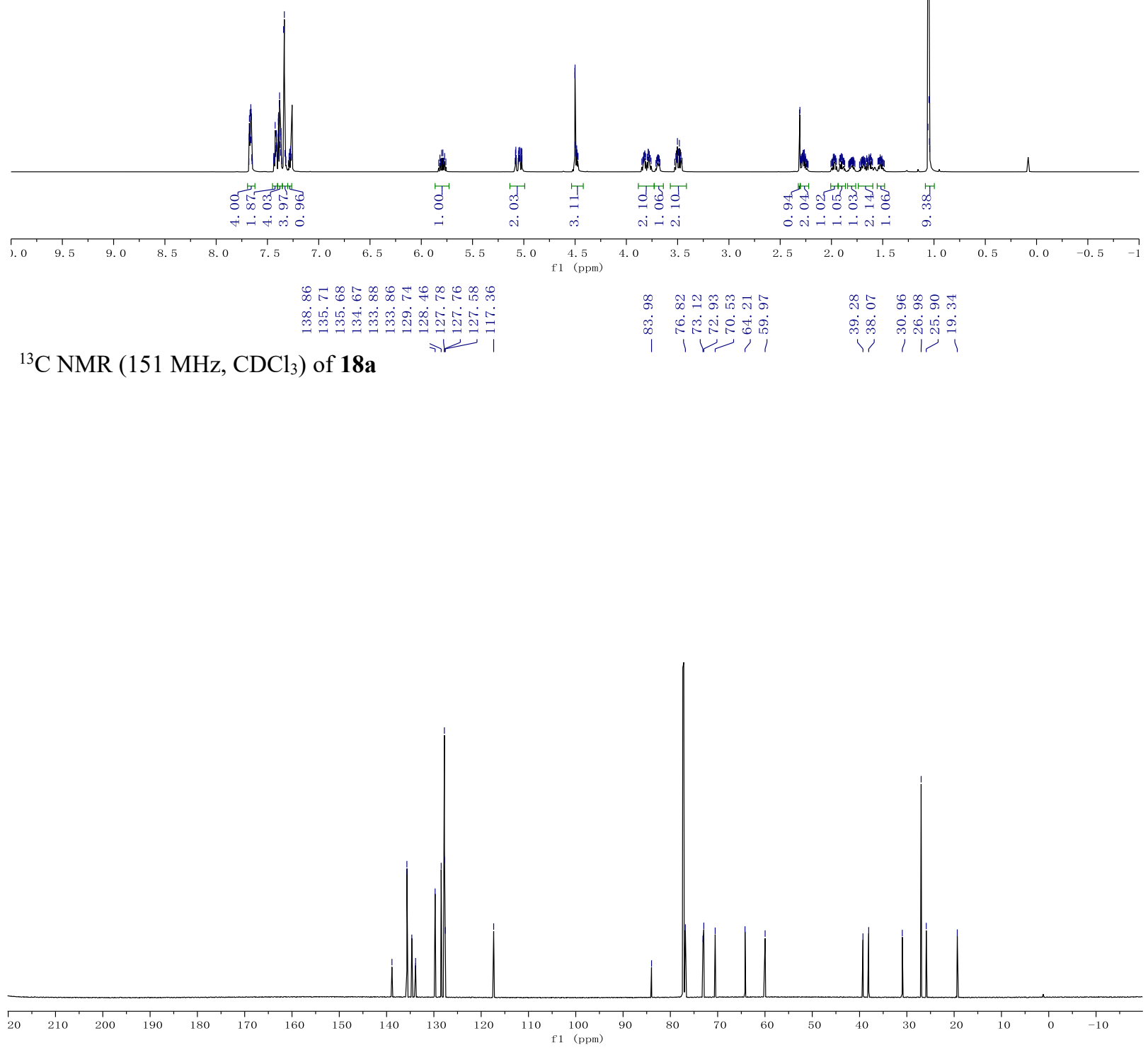
${ }^{1} \mathrm{H} \mathrm{NMR}\left(600 \mathrm{MHz}, \mathrm{CDCl}_{3}\right.$ ) of 20a
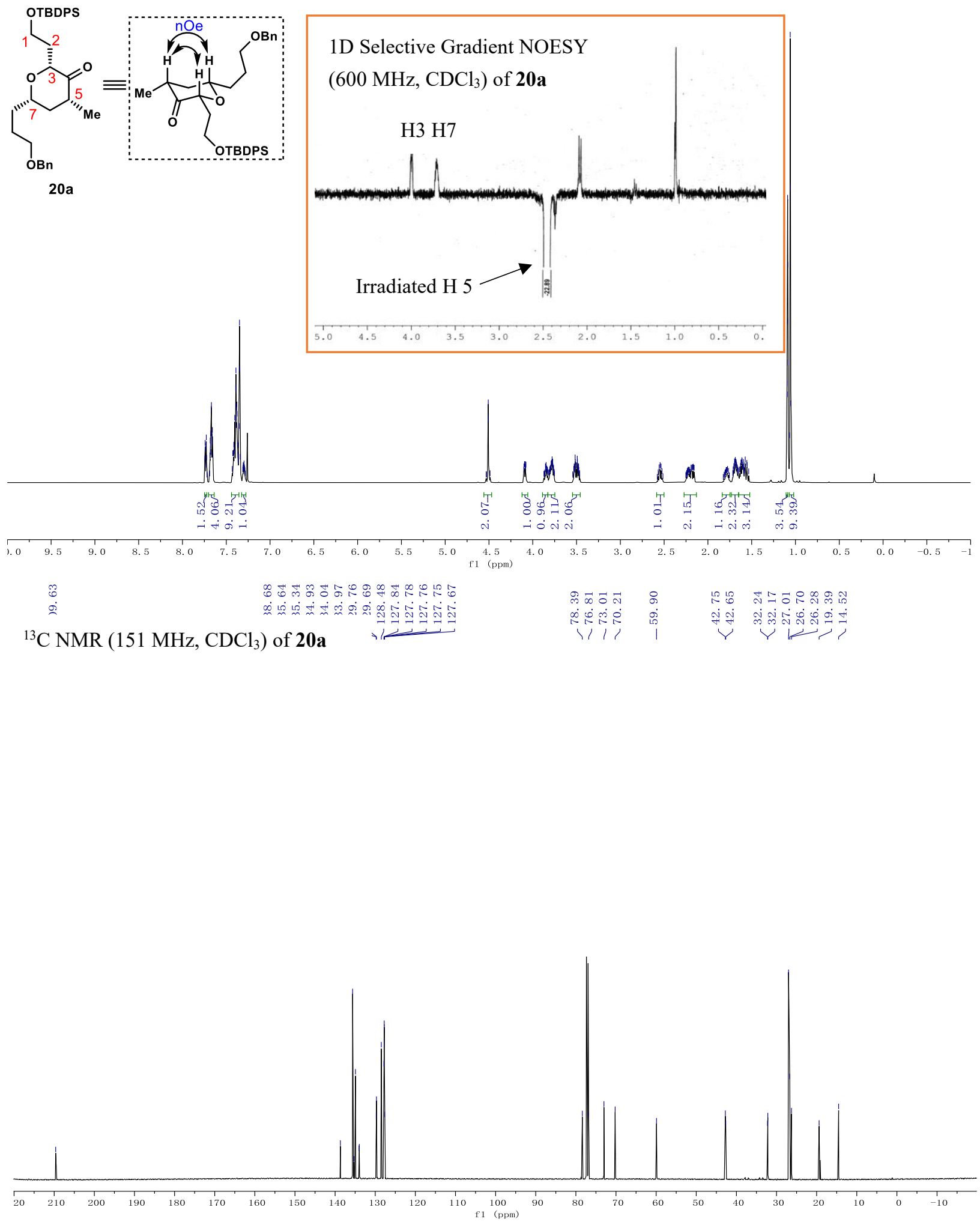
${ }^{1} \mathrm{H}$ NMR $\left(600 \mathrm{MHz}, \mathrm{CDCl}_{3}\right)$ of $\mathbf{1 9 b}$

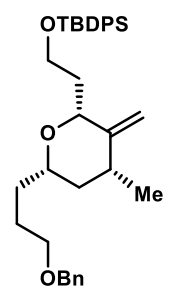

$19 \mathrm{~b}$
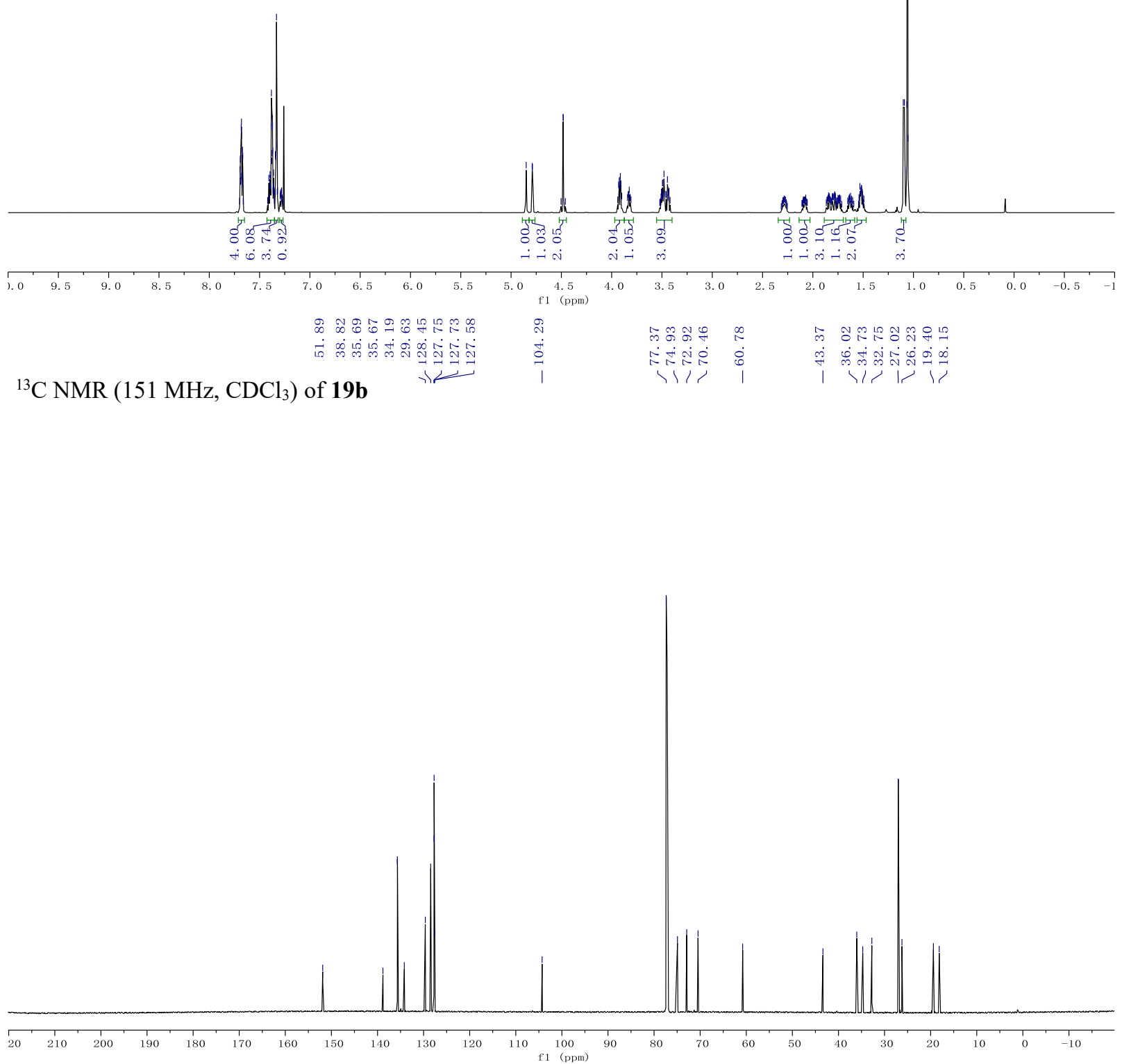
${ }^{1} \mathrm{H}$ NMR $\left(600 \mathrm{MHz}, \mathrm{CDCl}_{3}\right)$ of $\mathbf{S 1}$

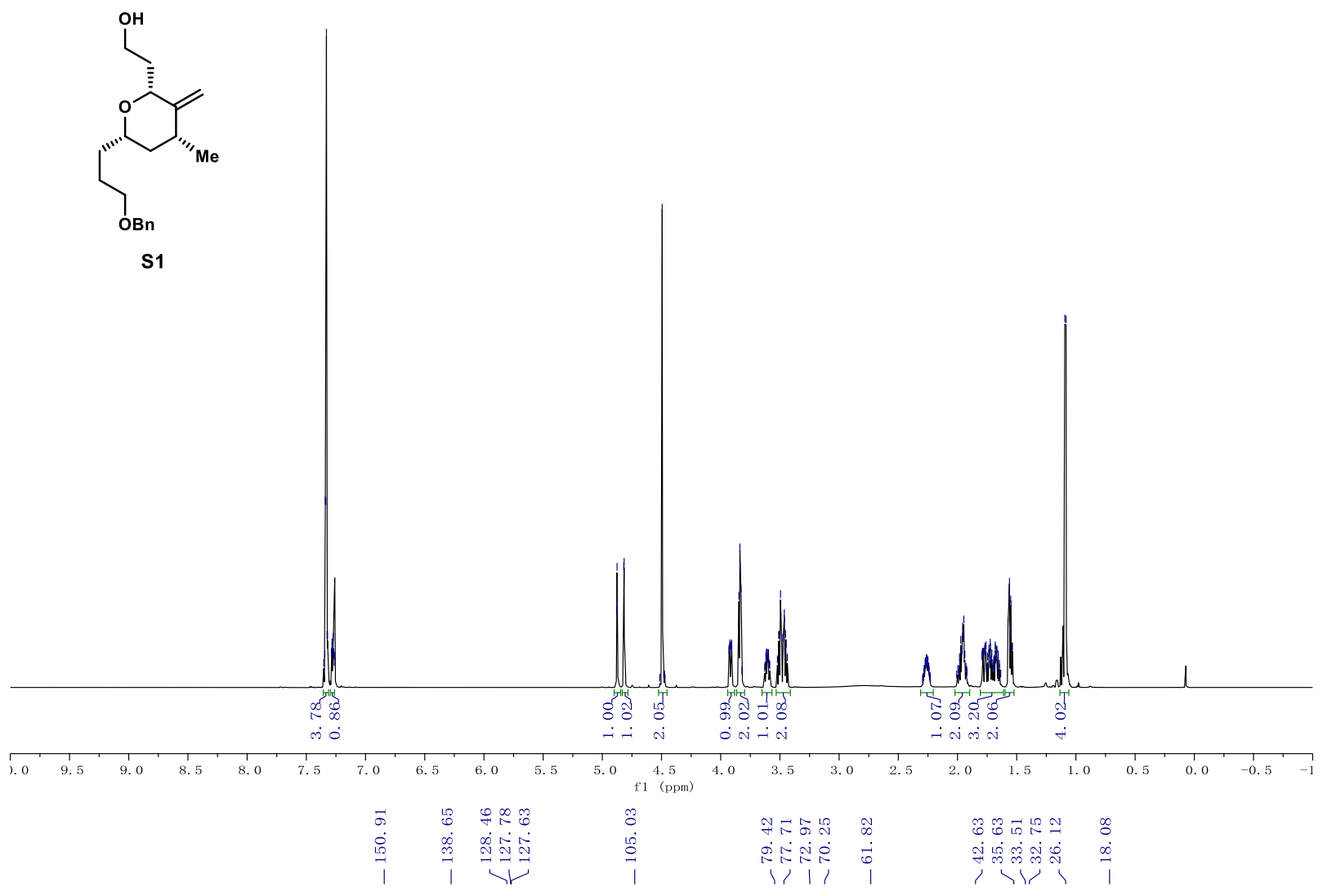

${ }^{13} \mathrm{C}$ NMR $\left(151 \mathrm{MHz}, \mathrm{CDCl}_{3}\right)$ of $\mathbf{S 1}$

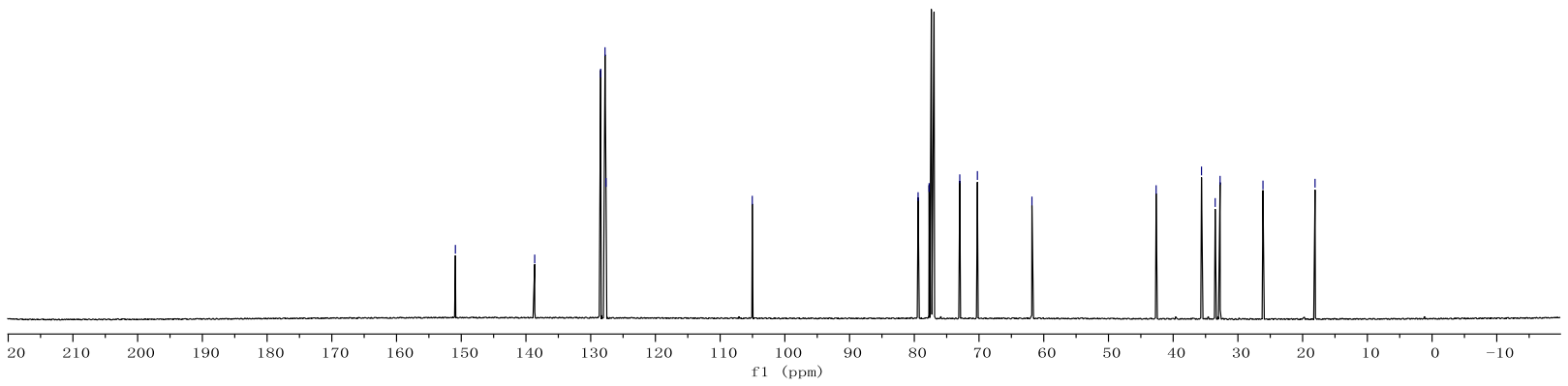



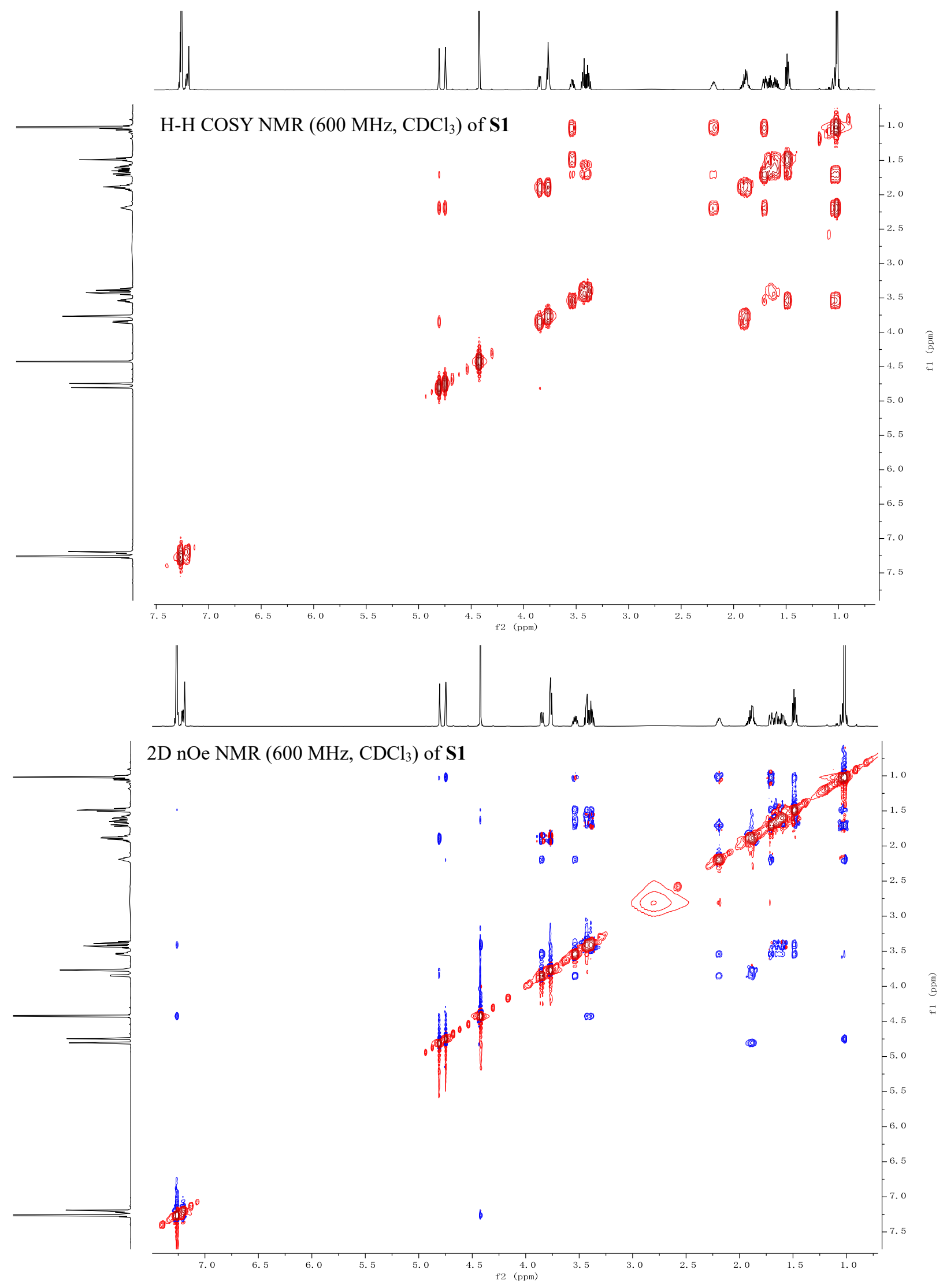
居

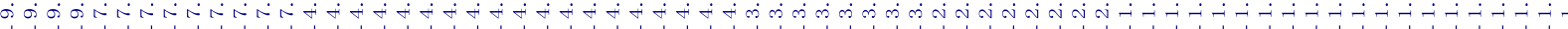

${ }^{1} \mathrm{H}$ NMR $\left(600 \mathrm{MHz}, \mathrm{CDCl}_{3}\right)$ of $\mathbf{2 1}$

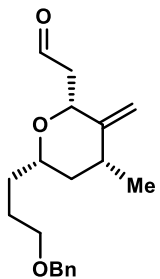

21

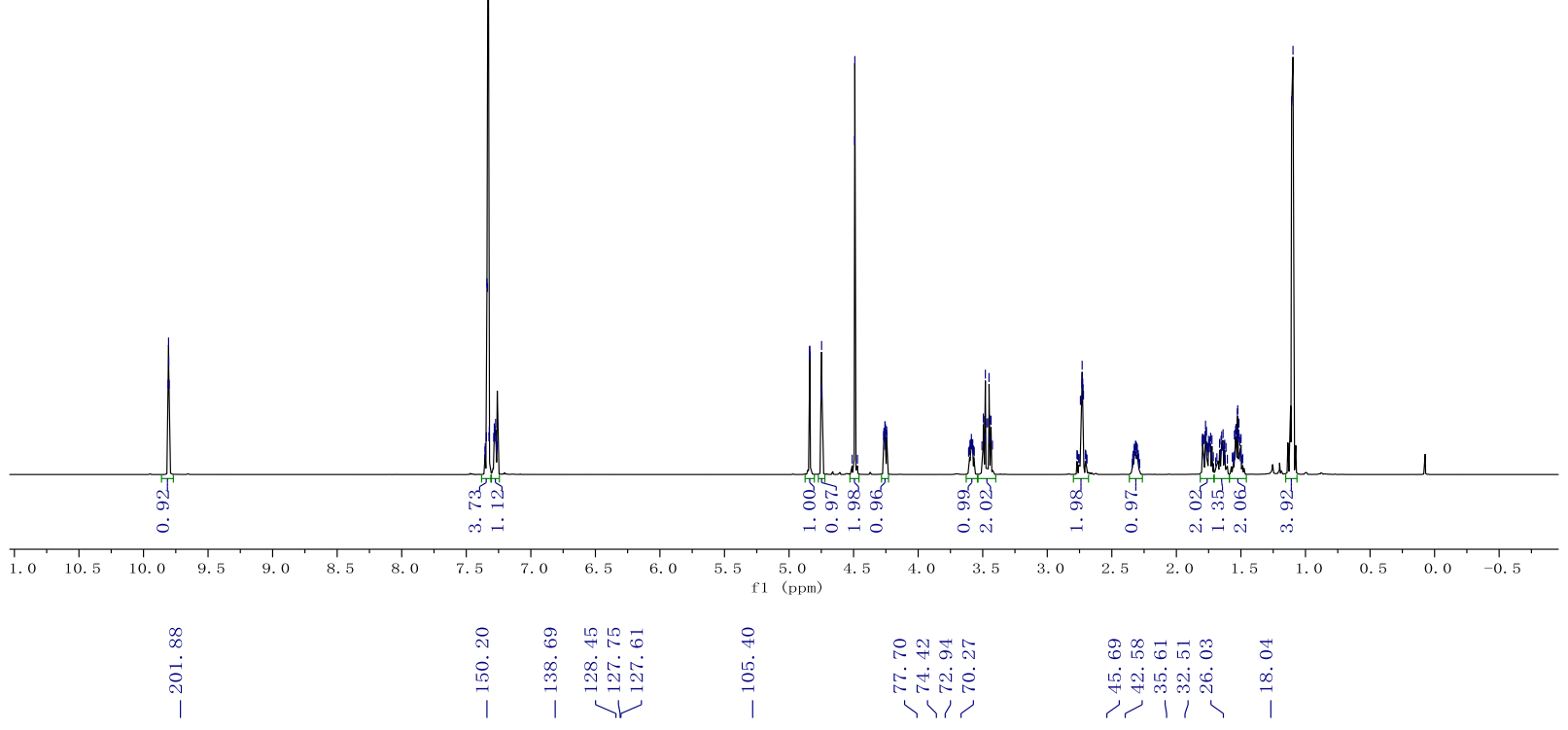

${ }^{13} \mathrm{C}$ NMR $\left(151 \mathrm{MHz}, \mathrm{CDCl}_{3}\right)$ of $\mathbf{2 1}$

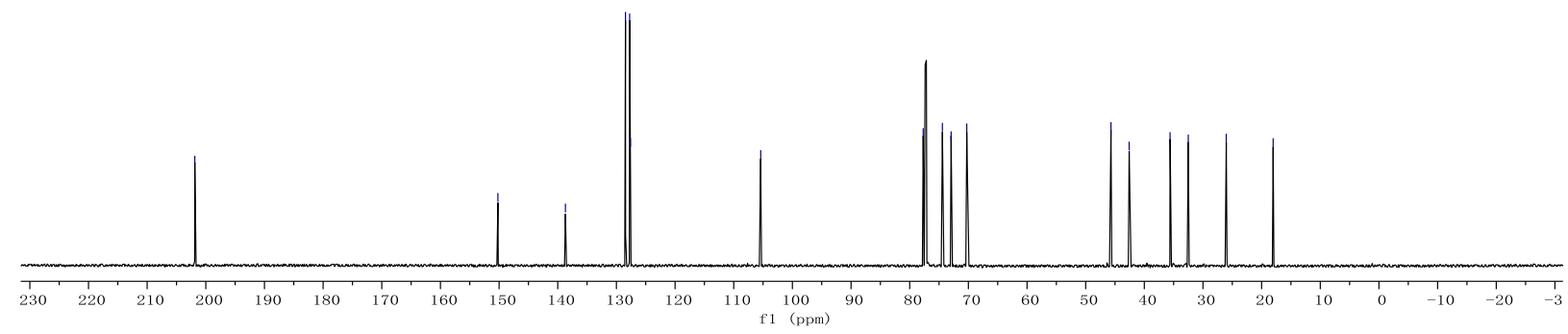


${ }^{1} \mathrm{H}$ NMR $\left(600 \mathrm{MHz}, \mathrm{CDCl}_{3}\right)$ of $\mathbf{2 2}$

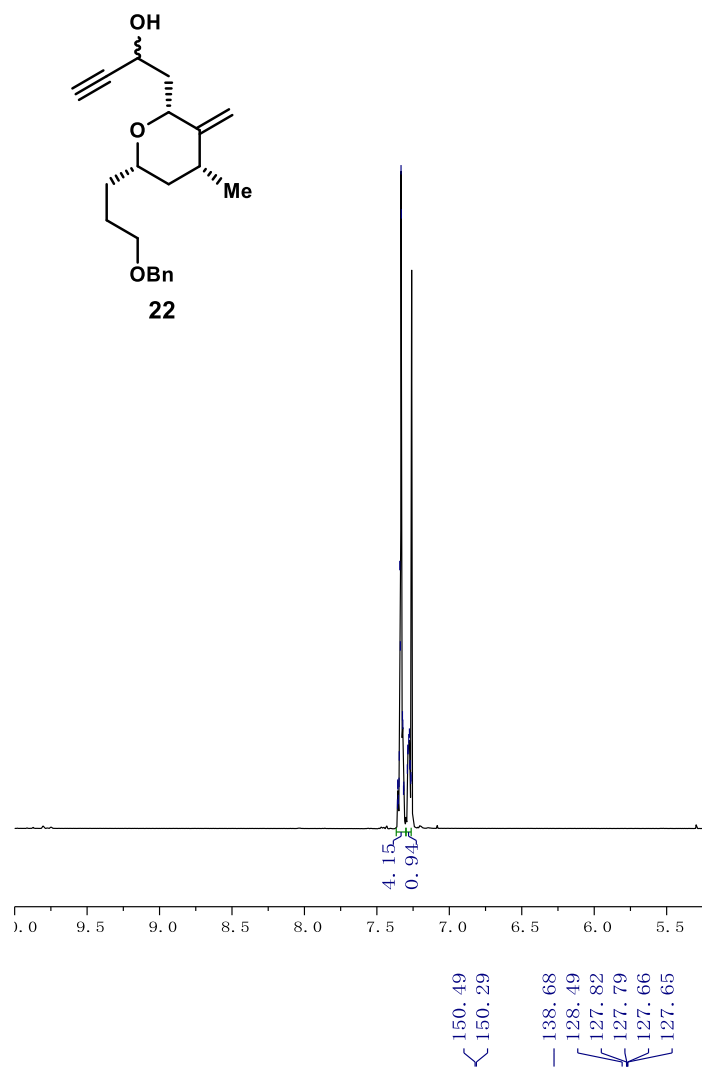

${ }^{13} \mathrm{C} \mathrm{NMR}\left(151 \mathrm{MHz}, \mathrm{CDCl}_{3}\right)$ of 22
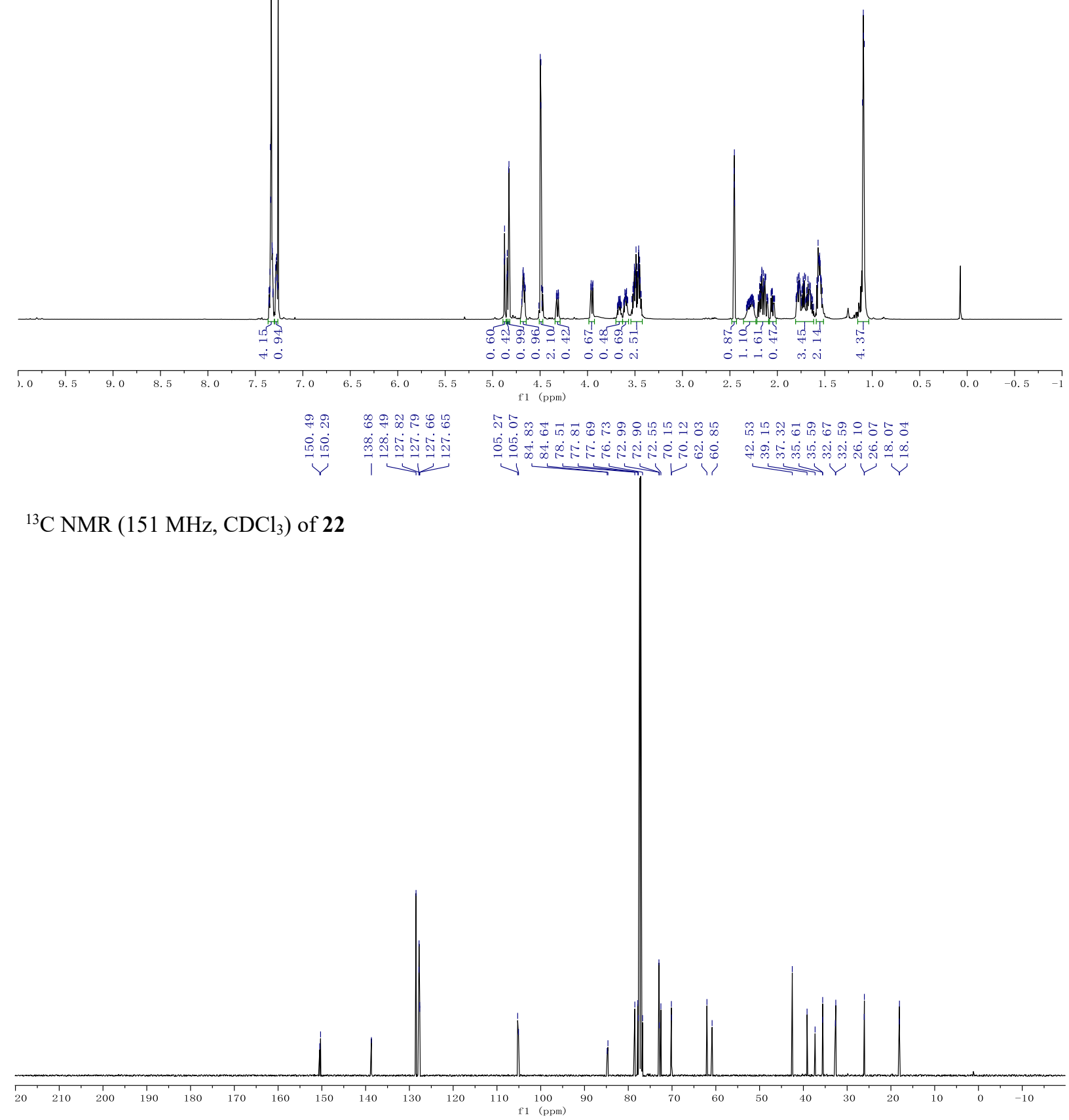


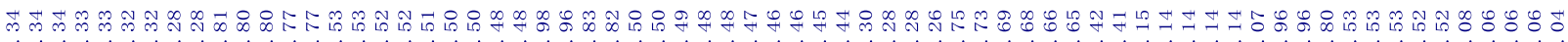

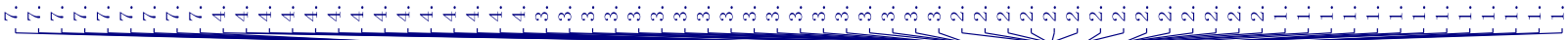

${ }^{1} \mathrm{H}$ NMR $\left(600 \mathrm{MHz}, \mathrm{CDCl}_{3}\right)$ of $\mathbf{2 4 a}$

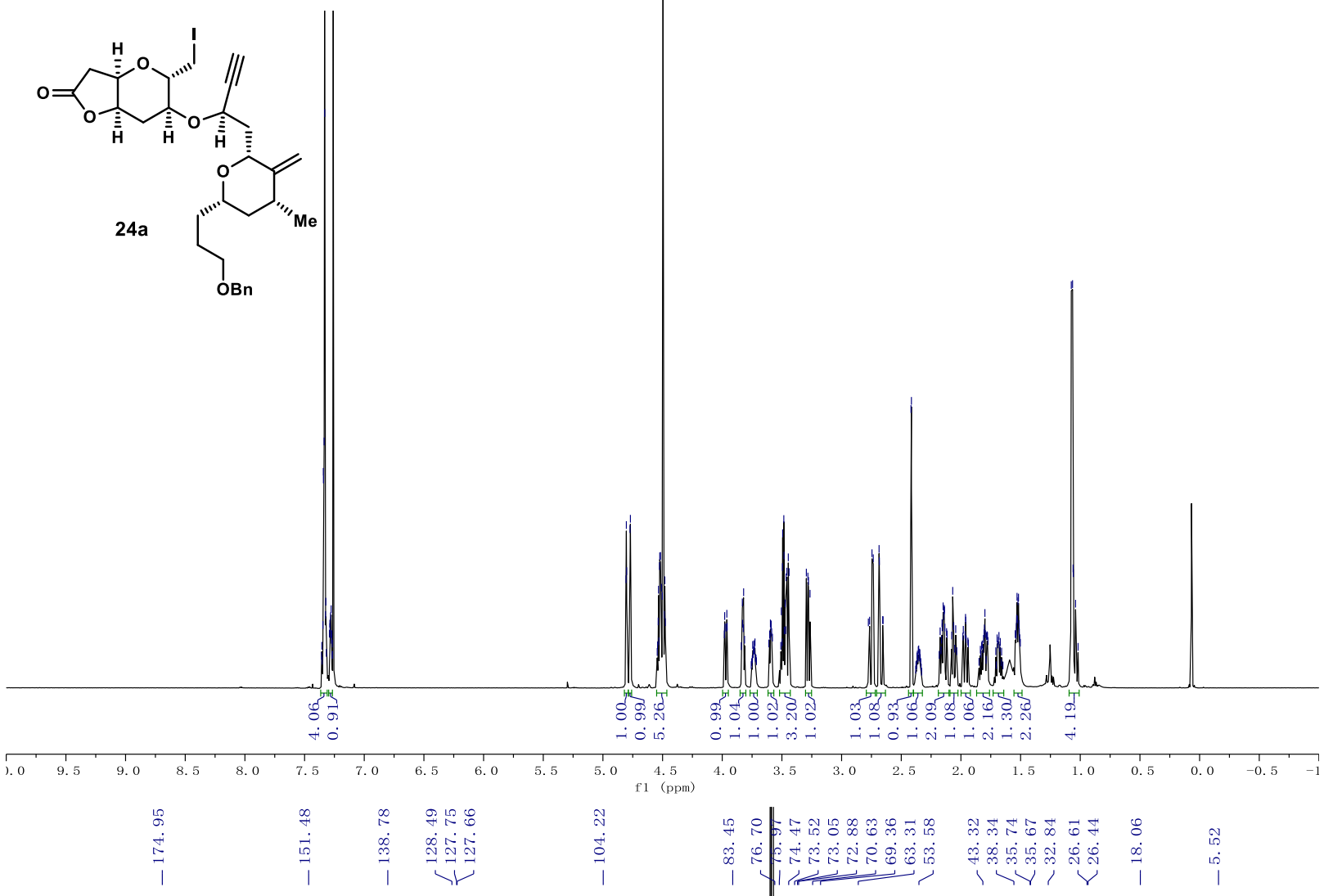

${ }^{13} \mathrm{C} \mathrm{NMR}\left(151 \mathrm{MHz}, \mathrm{CDCl}_{3}\right)$ of $\mathbf{2 4 a}$

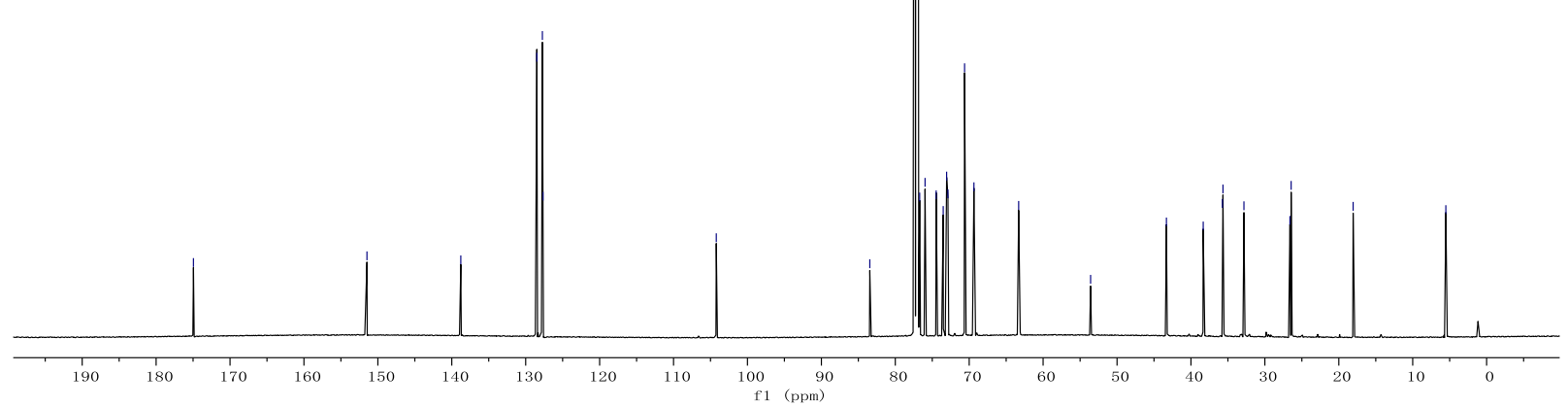


${ }^{1} \mathrm{H}$ NMR $\left(600 \mathrm{MHz}, \mathrm{CDCl}_{3}\right)$ of $\mathbf{2 4 b}$
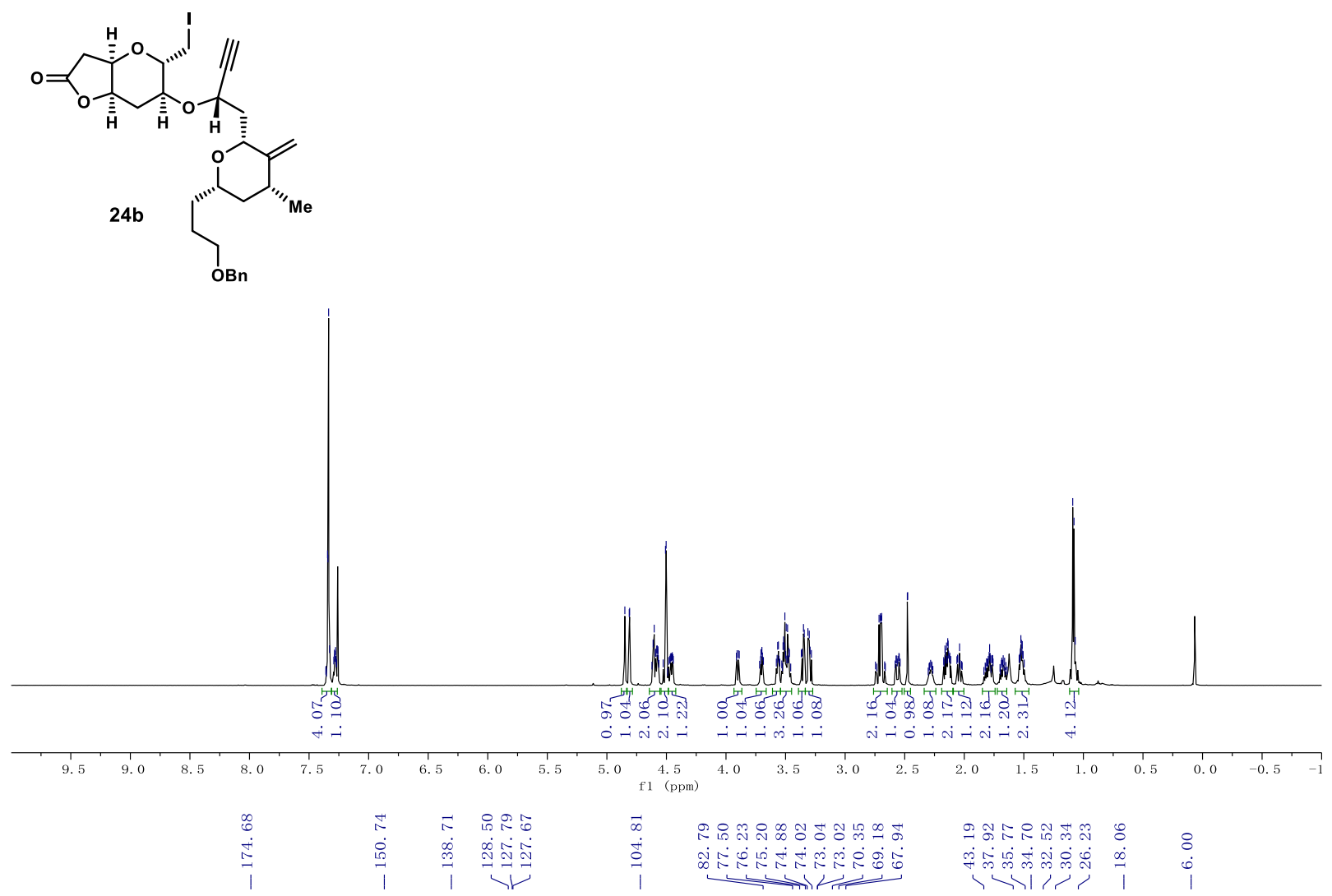

${ }^{13} \mathrm{C}$ NMR $\left(151 \mathrm{MHz}, \mathrm{CDCl}_{3}\right)$ of $\mathbf{2 4 b}$

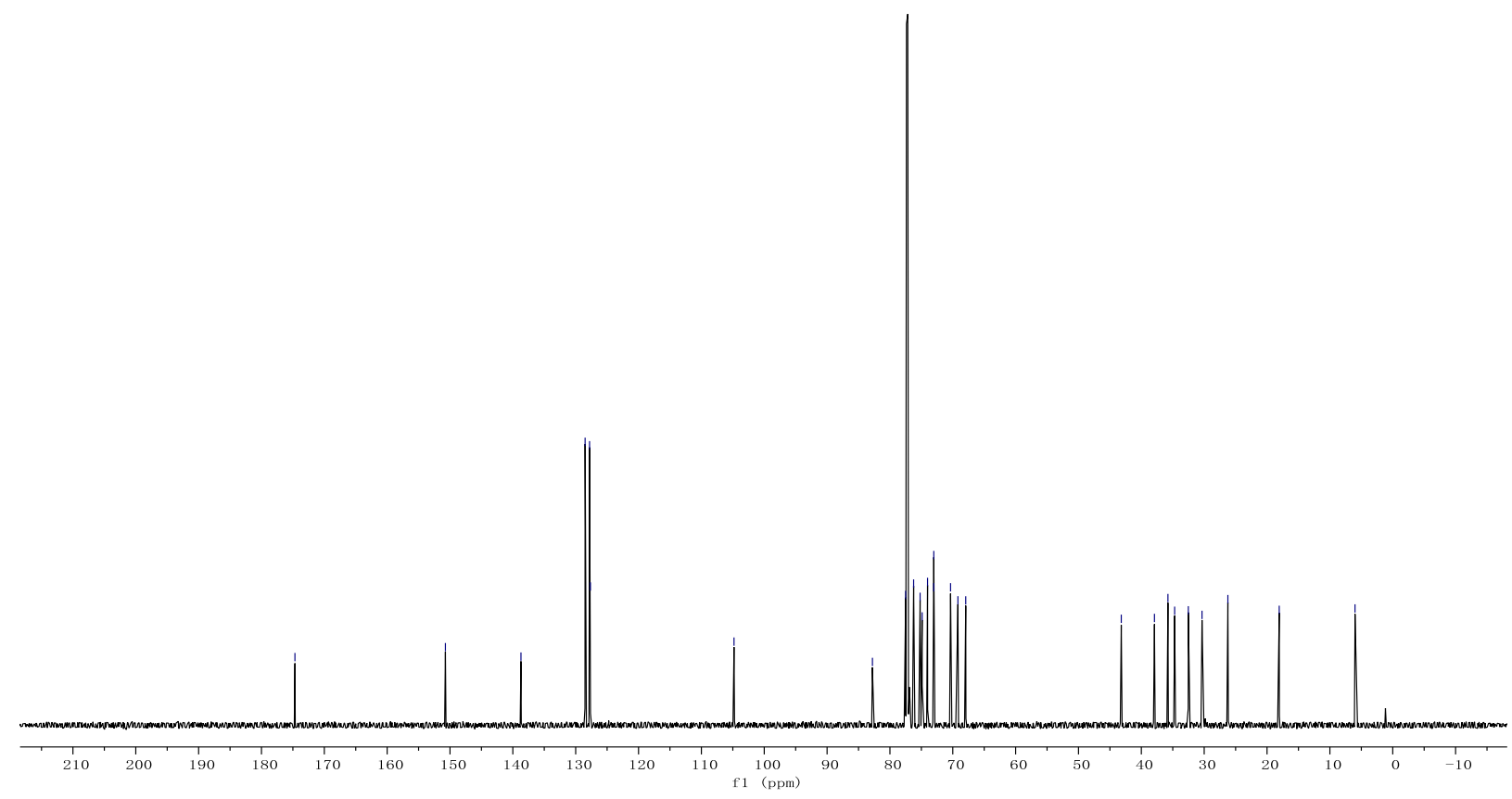




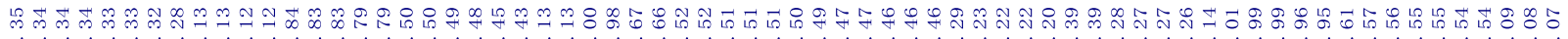
ヘ

${ }^{1} \mathrm{H}$ NMR $\left(600 \mathrm{MHz}, \mathrm{CDCl}_{3}\right)$ of $\mathbf{2 5}$
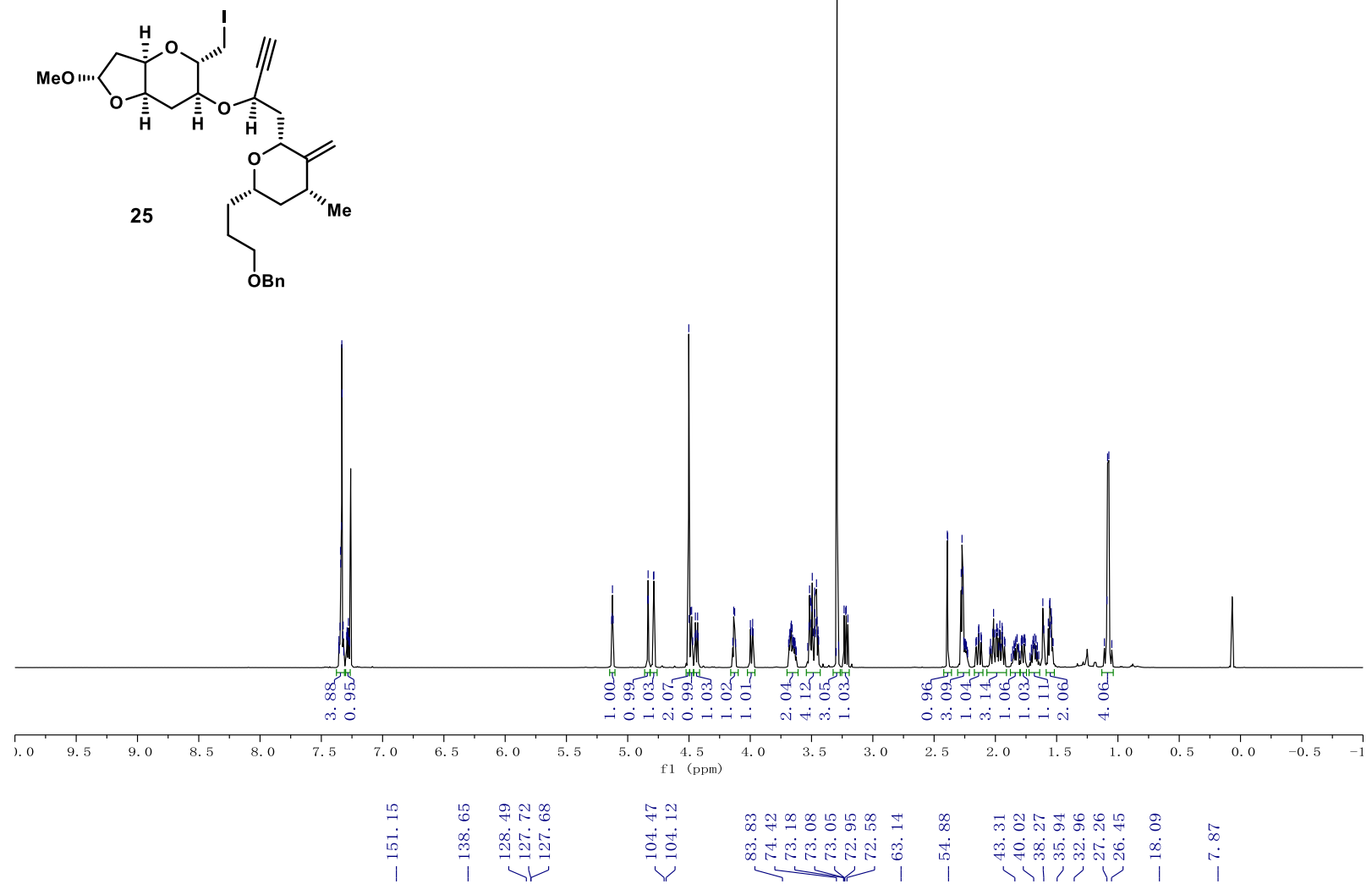

${ }^{13} \mathrm{C}$ NMR $\left(151 \mathrm{MHz}, \mathrm{CDCl}_{3}\right)$ of $\mathbf{2 5}$

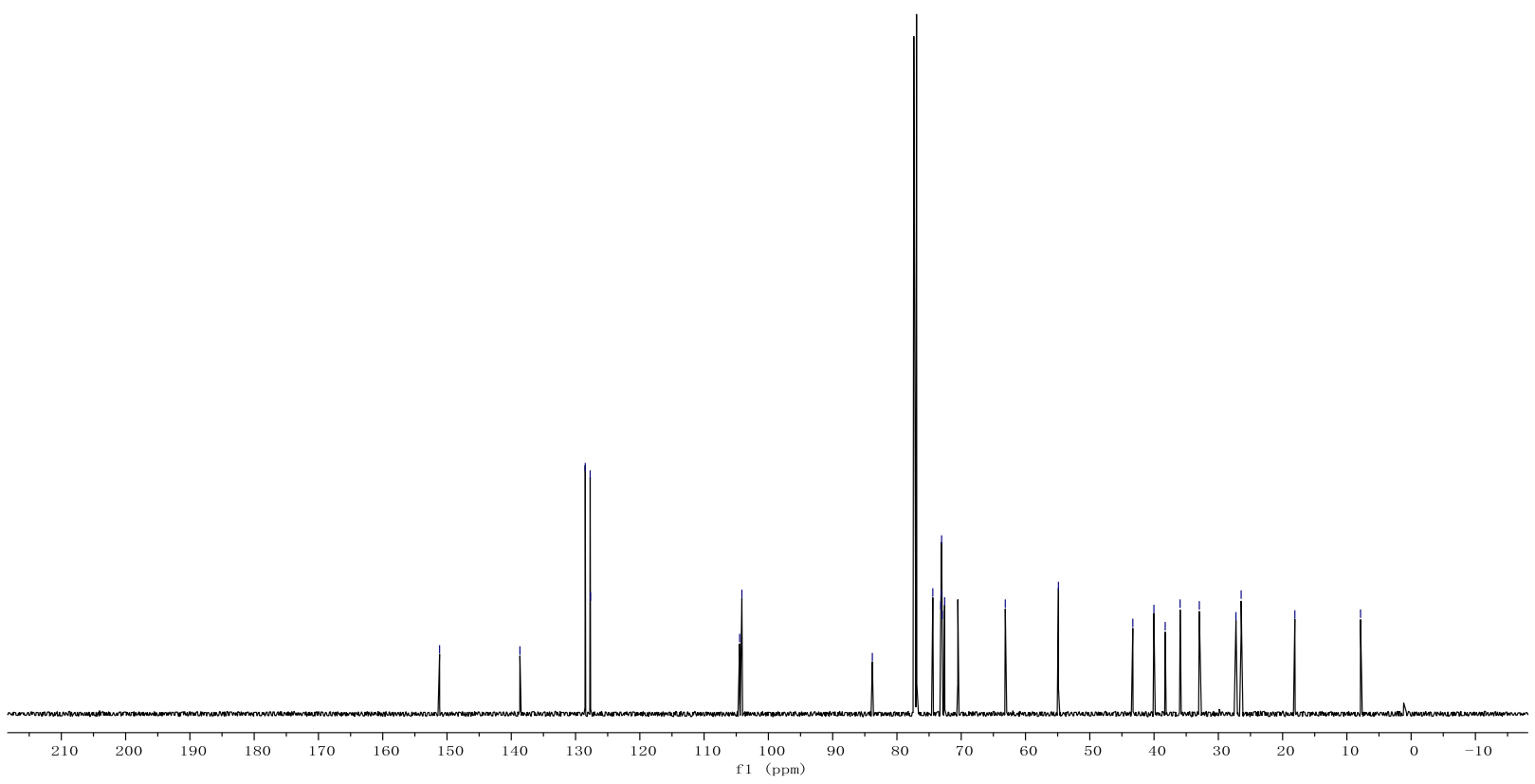


${ }^{1} \mathrm{H}$ NMR $\left(600 \mathrm{MHz}, \mathrm{C}_{6} \mathrm{D}_{6}\right)$ of 27

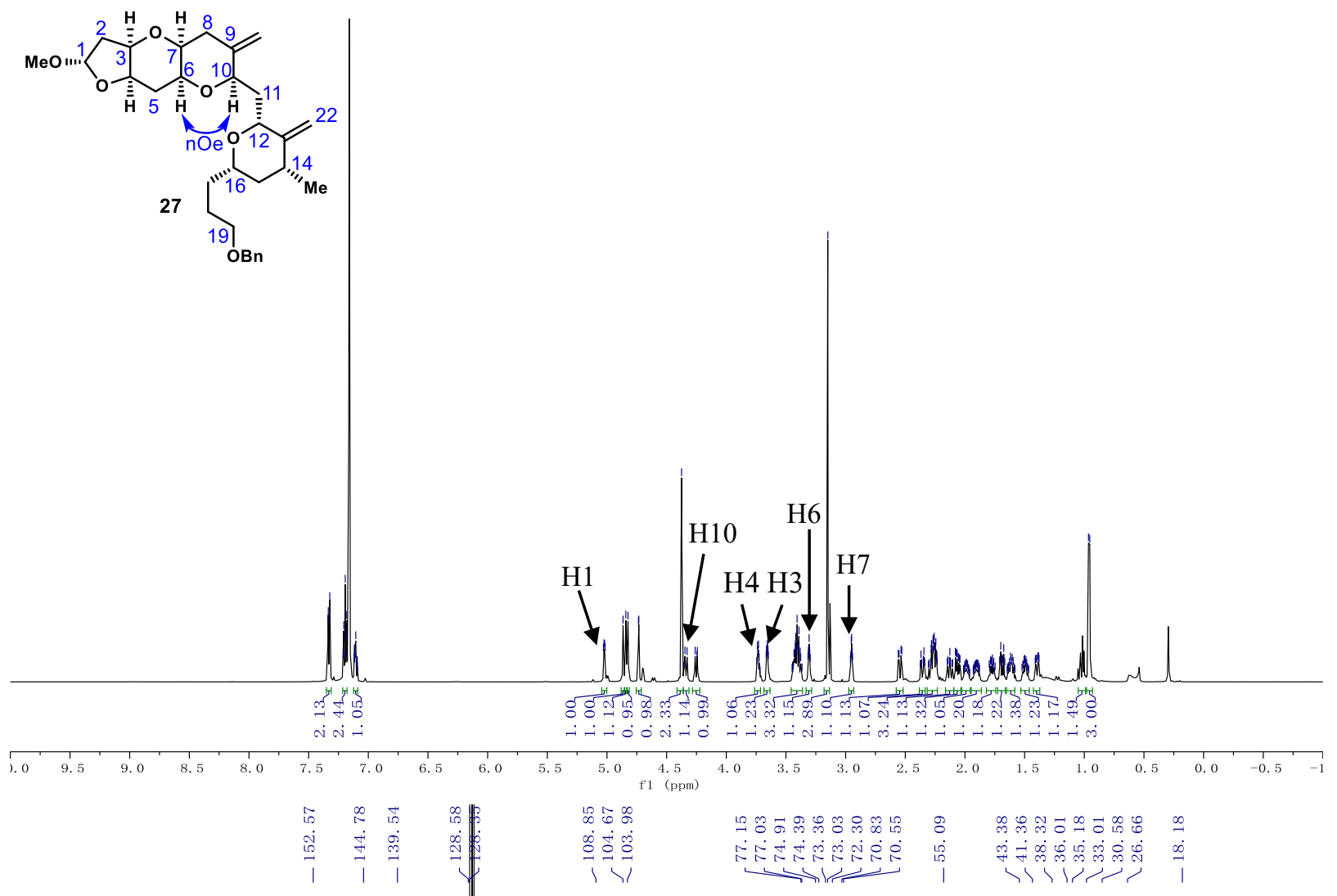

${ }^{13} \mathrm{C}$ NMR (151 MHz, $\left.\mathrm{C}_{6} \mathrm{D}_{6}\right)$ of 27

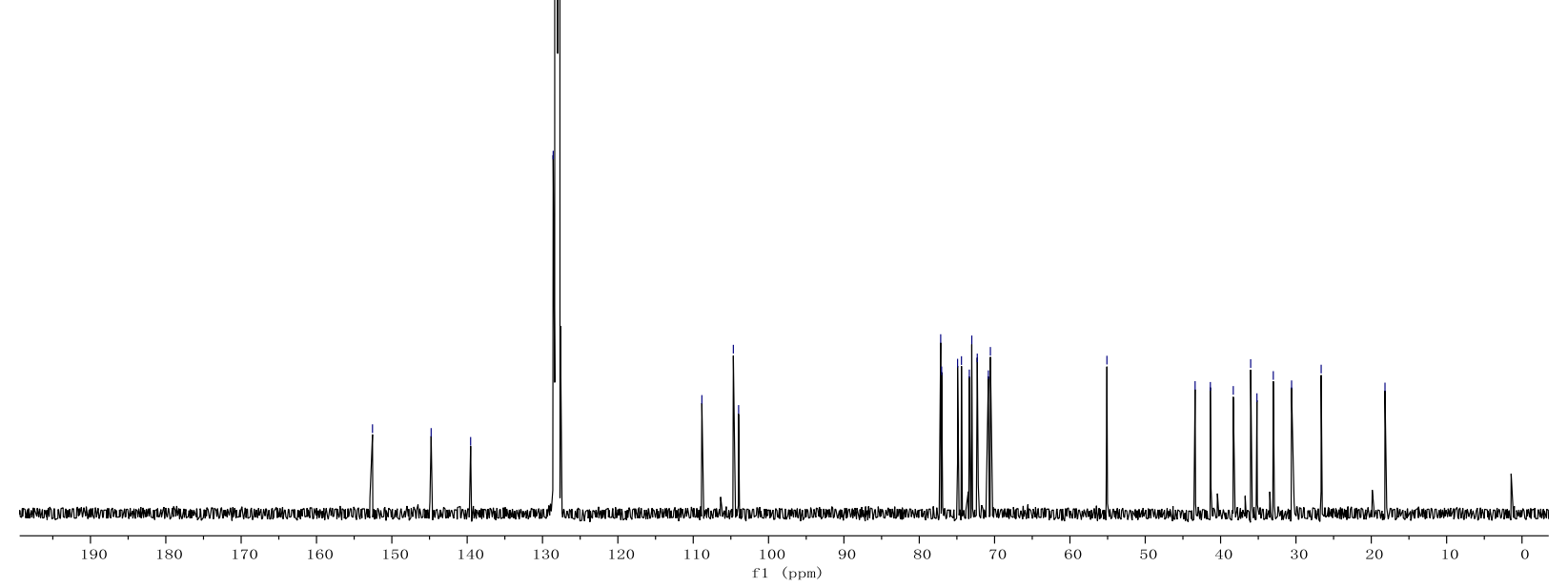




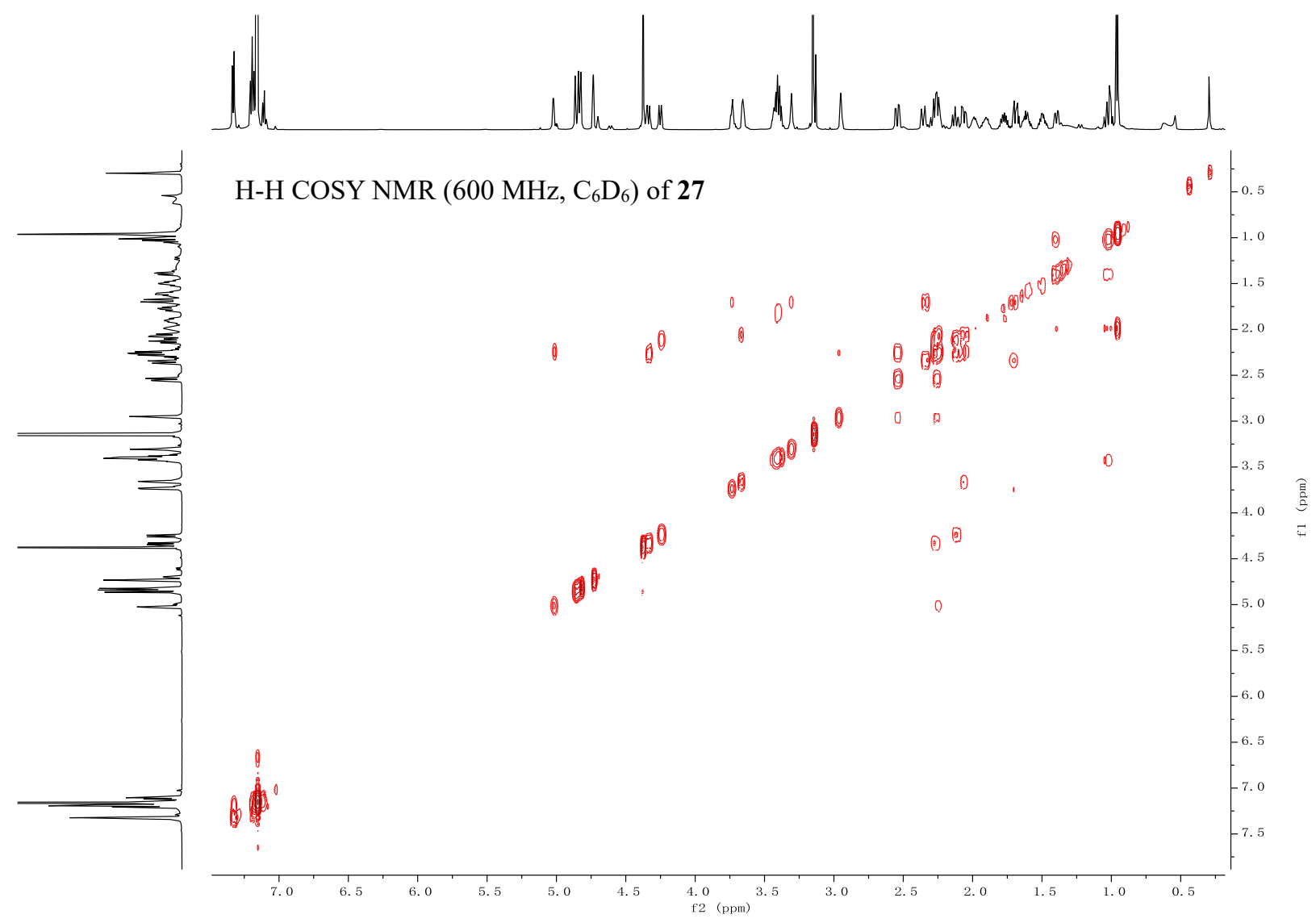

1D Selective Gradient NOESY (600 MHz, $\mathrm{C}_{6} \mathrm{D}_{6}$ ) of 27

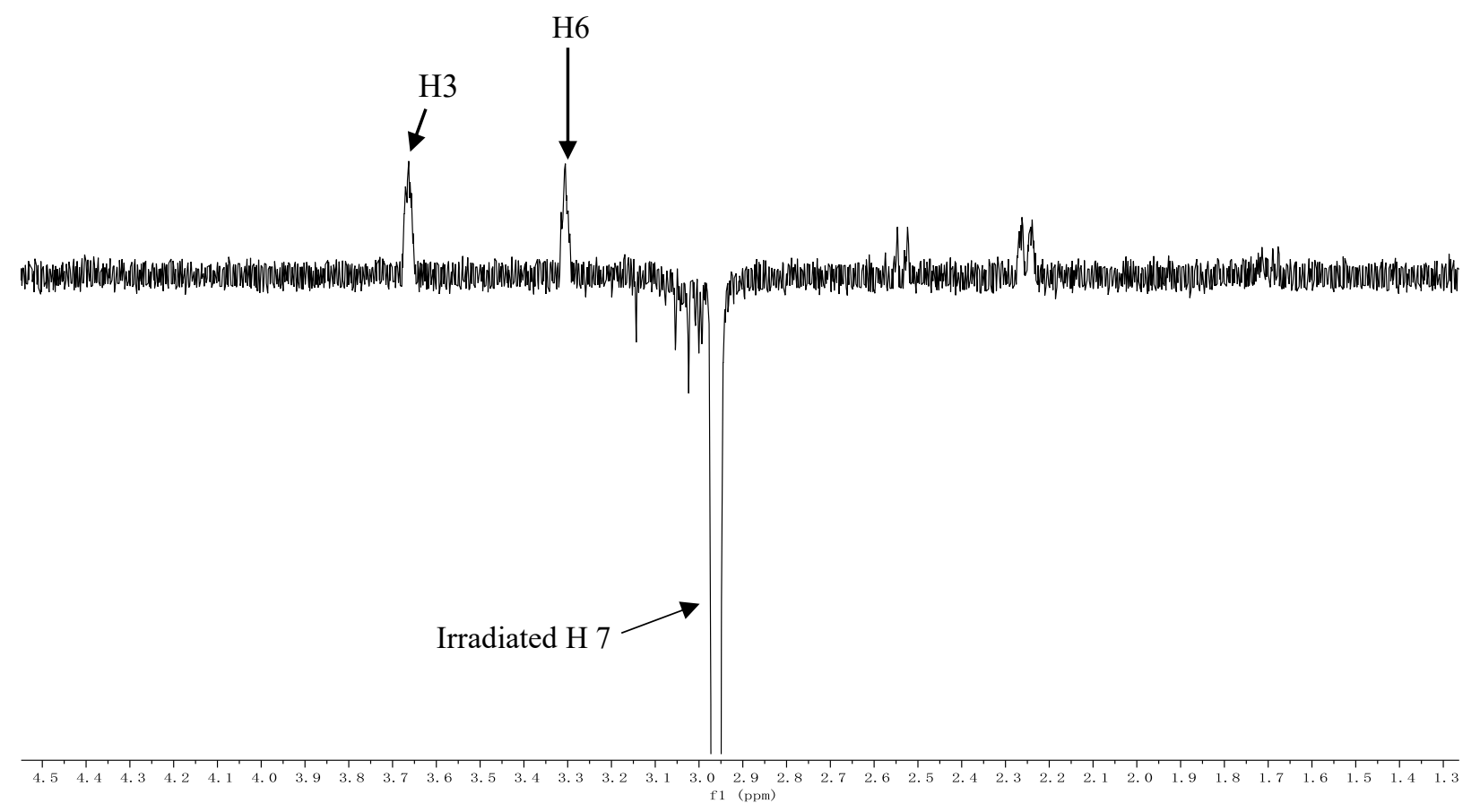


1D Selective Gradient NOESY (600 MHz, $\left.\mathrm{C}_{6} \mathrm{D}_{6}\right)$ of 27

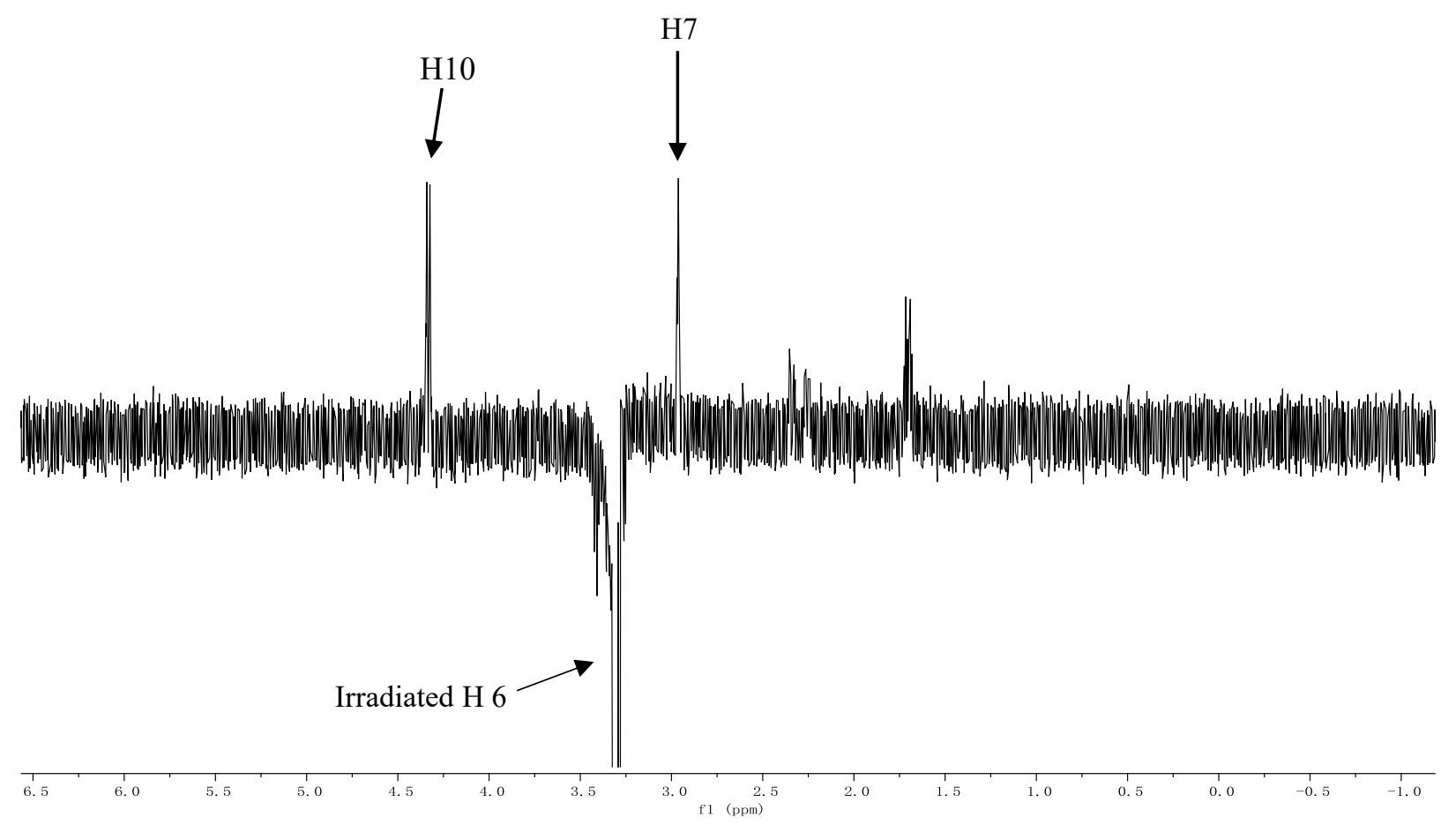

1D Selective Gradient NOESY (600 MHz, $\left.\mathrm{C}_{6} \mathrm{D}_{6}\right)$ of 27

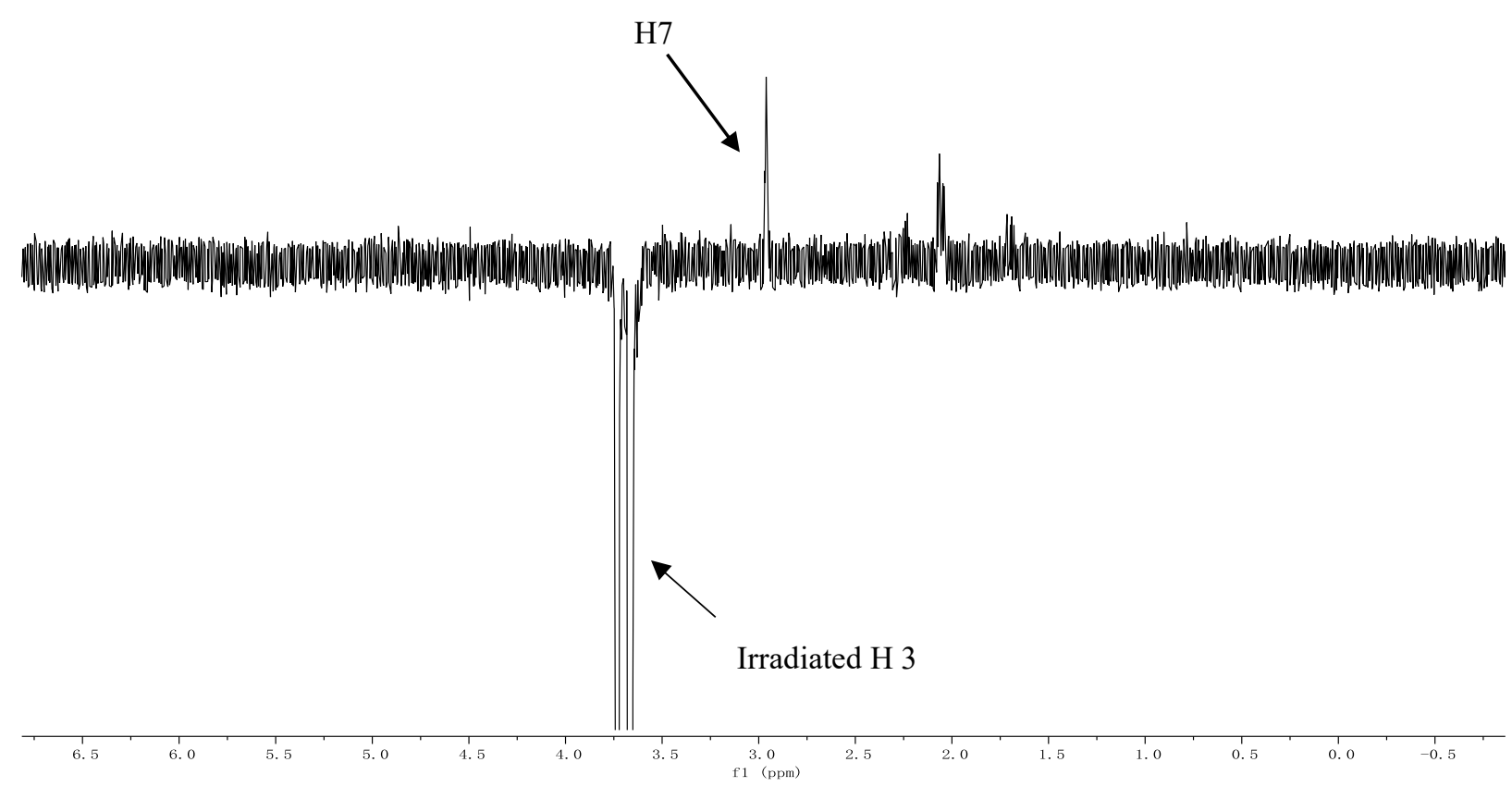


${ }^{1} \mathrm{H}$ NMR (600 MHz, $\left.\mathrm{C}_{6} \mathrm{D}_{6}\right)$ of $\mathbf{2 8}$
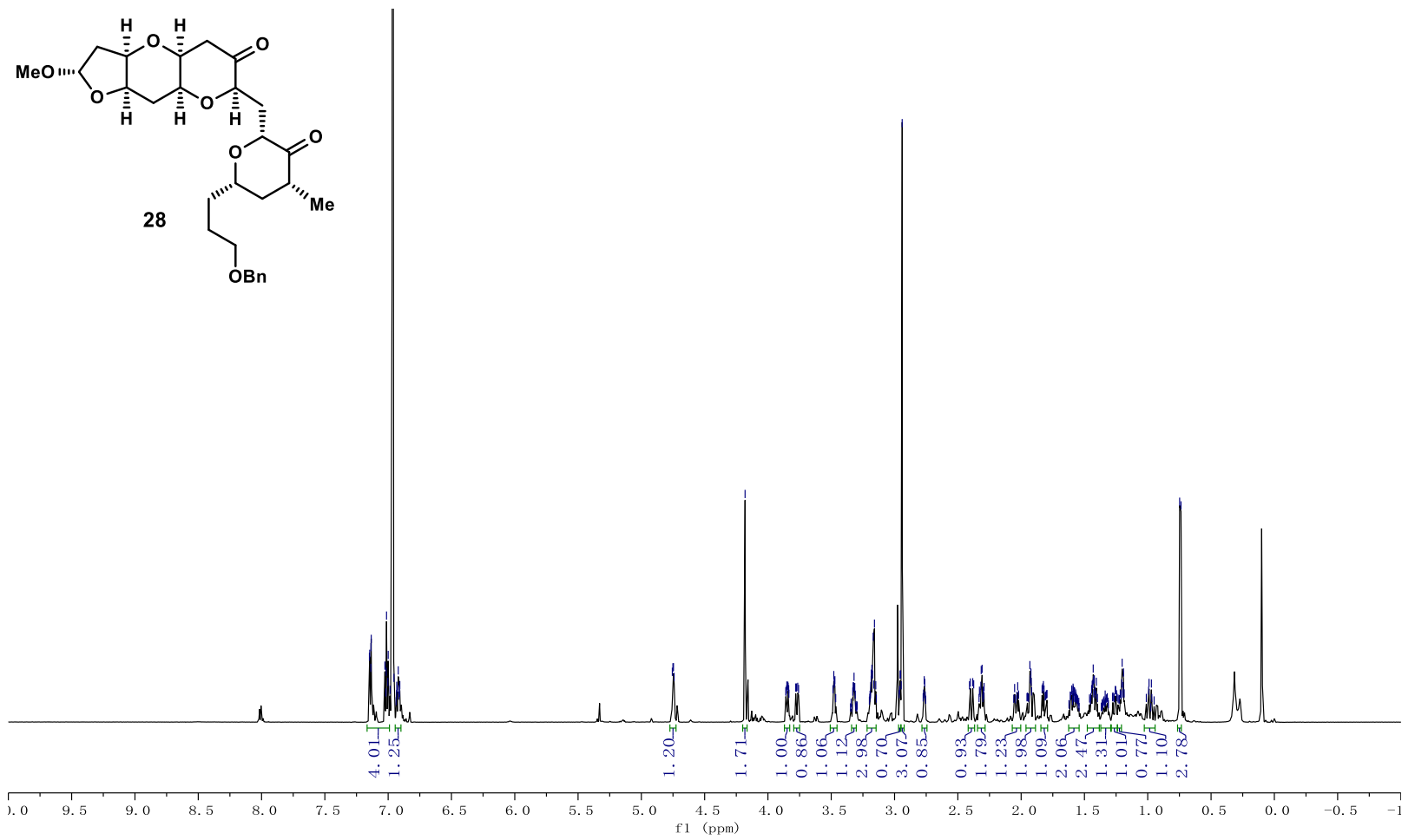

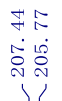

${ }^{13} \mathrm{C}$ NMR (151 MHz, $\left.\mathrm{C}_{6} \mathrm{D}_{6}\right)$ of 28

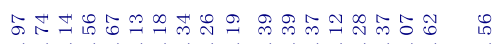

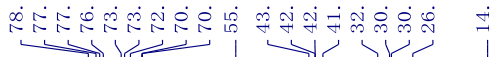

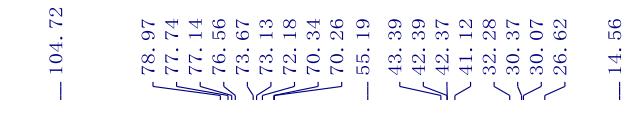




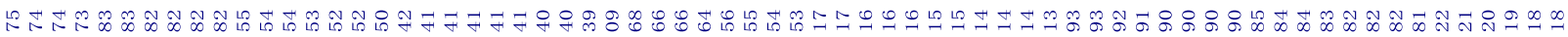

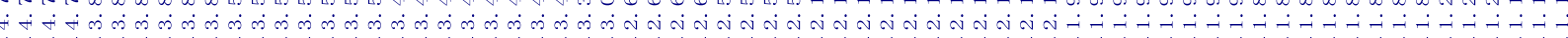

${ }^{1} \mathrm{H}$ NMR $\left(600 \mathrm{MHz}, \mathrm{C}_{6} \mathrm{D}_{6}\right)$ of $\mathbf{S 2}$
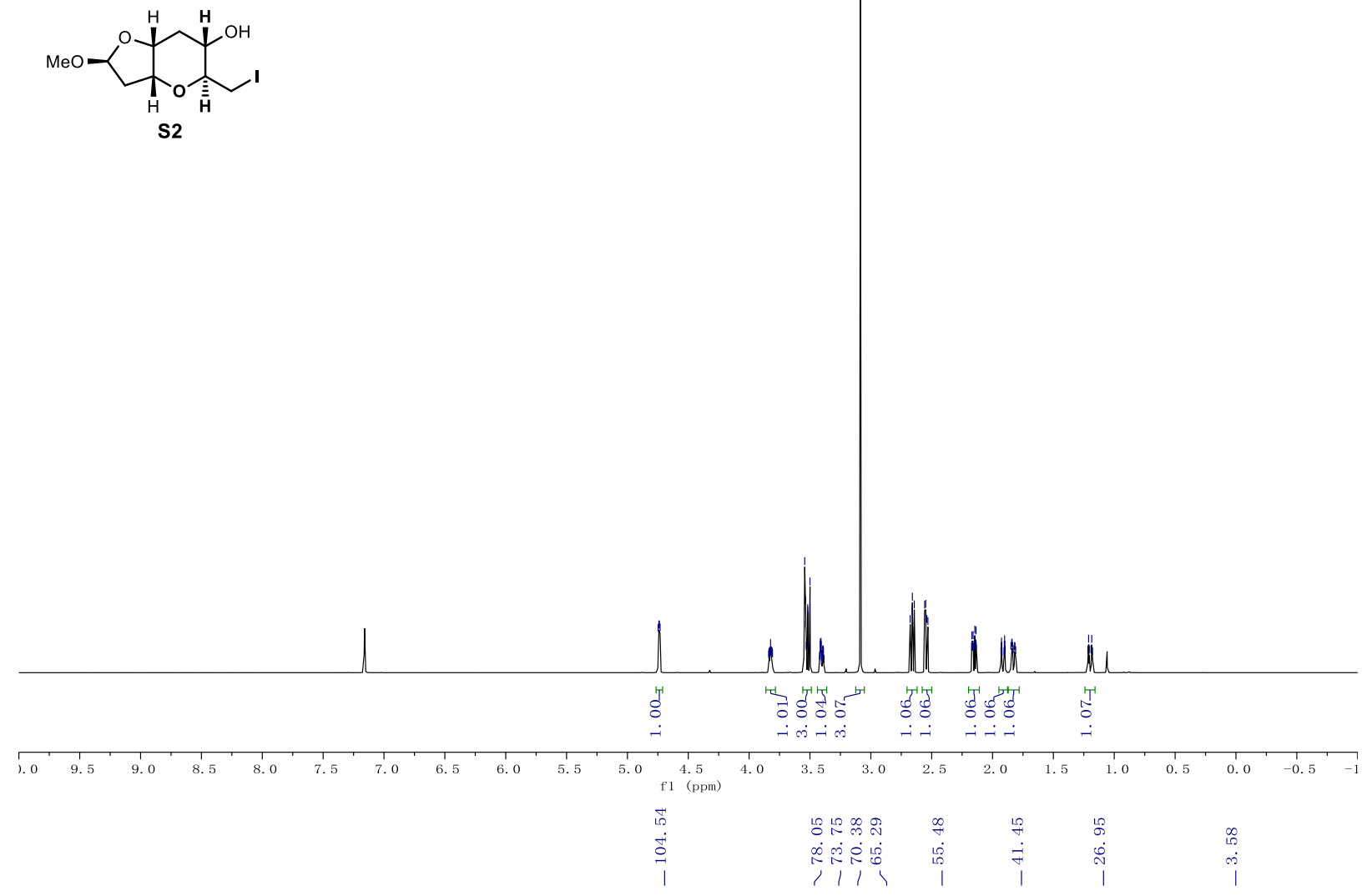

${ }^{13} \mathrm{C}$ NMR (151 MHz, $\left.\mathrm{C}_{6} \mathrm{D}_{6}\right)$ of $\mathbf{S 2}$

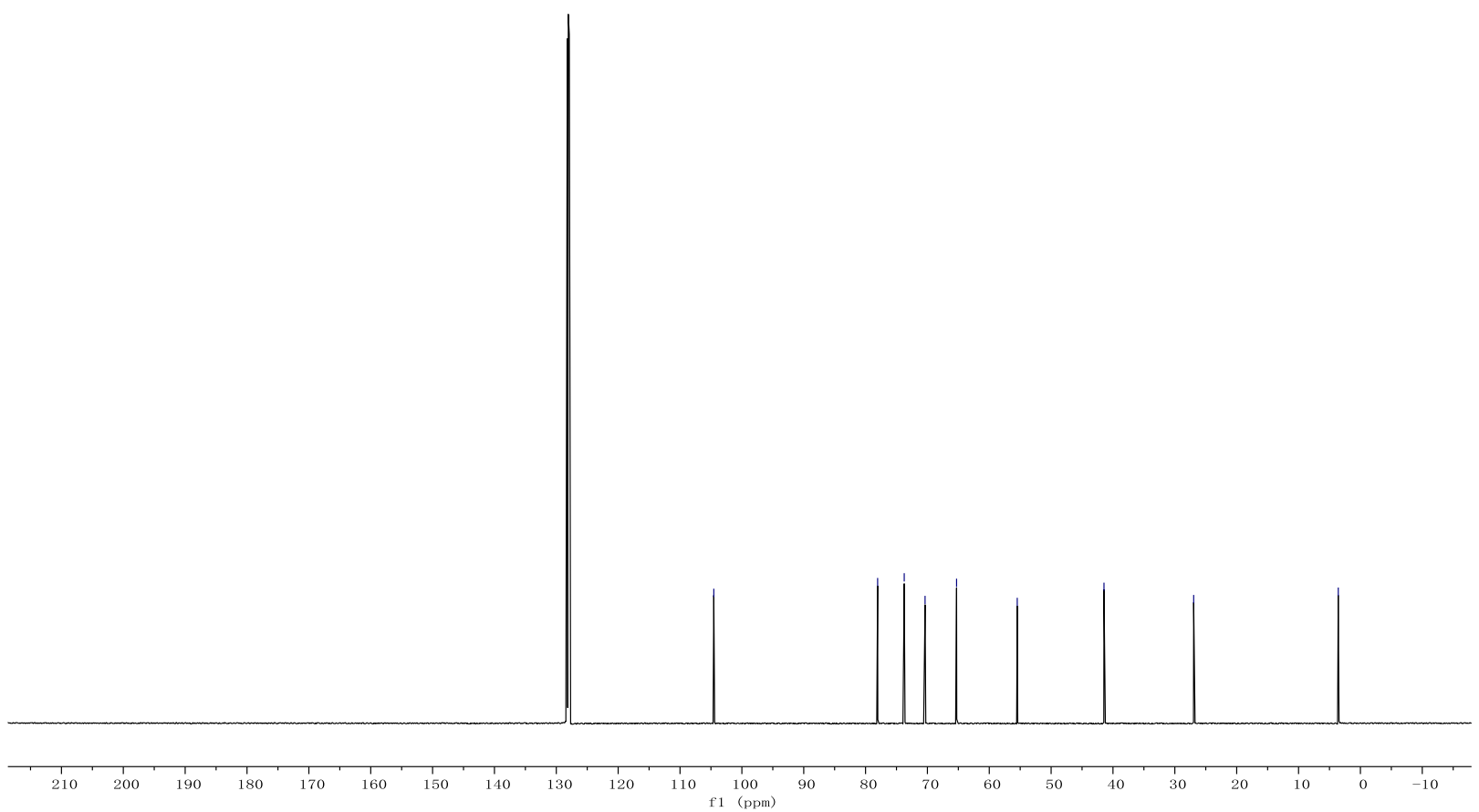



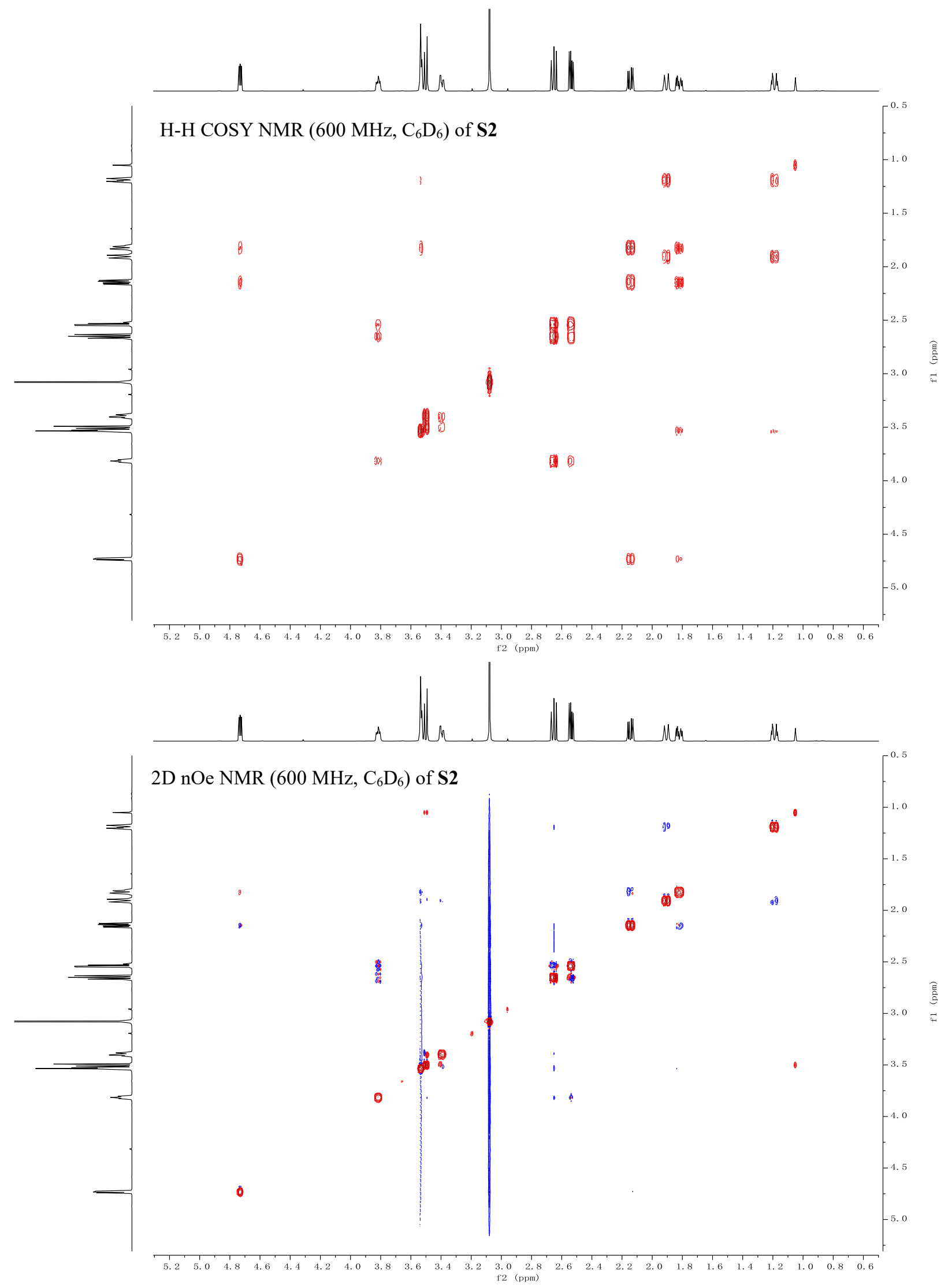
${ }^{1} \mathrm{H}$ NMR $\left(600 \mathrm{MHz}, \mathrm{CDCl}_{3}\right)$ of $\mathbf{3 4}$
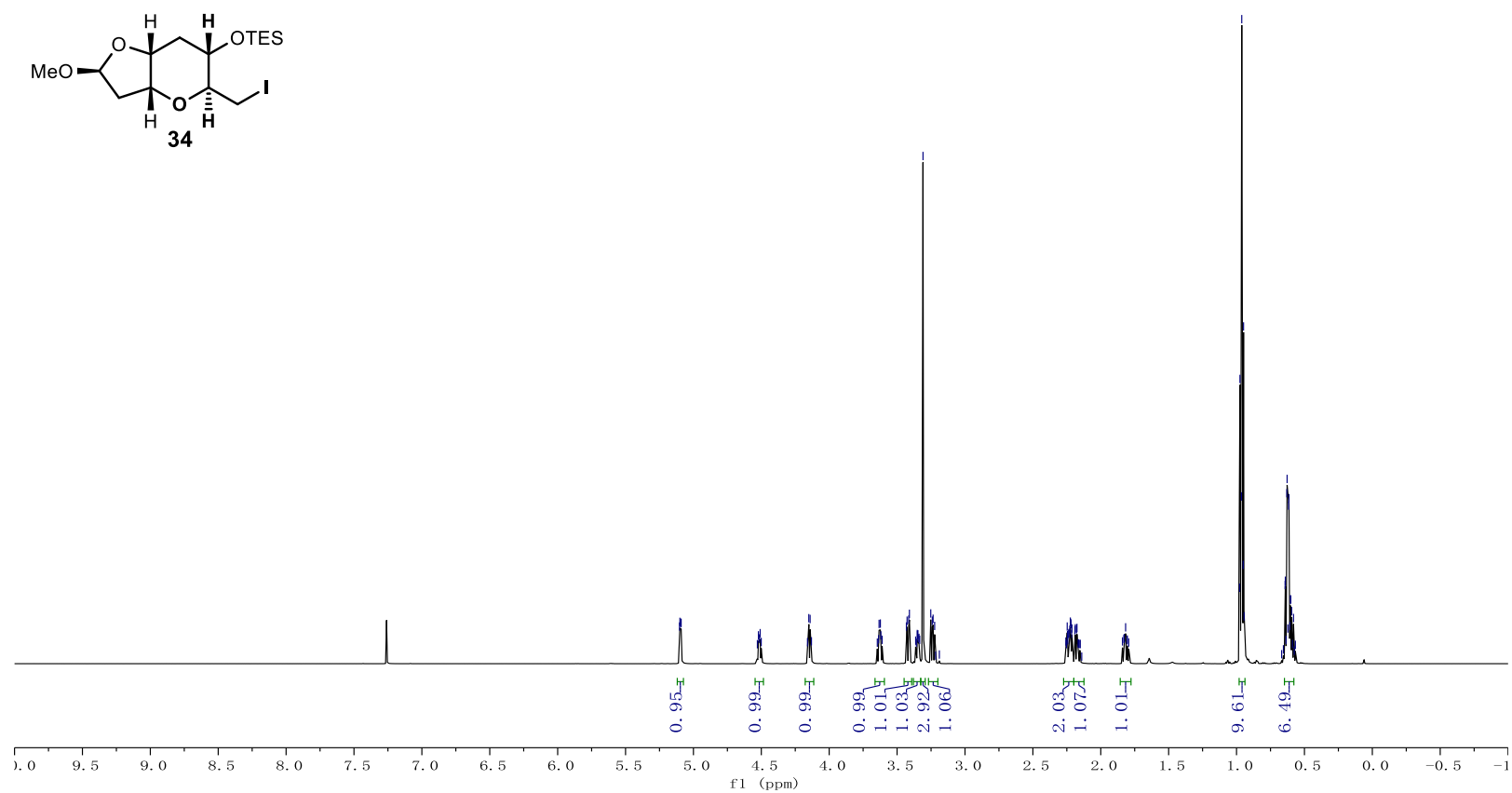

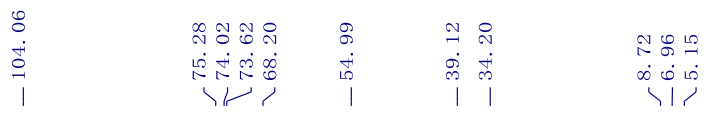

${ }^{13} \mathrm{C}$ NMR $\left(151 \mathrm{MHz}, \mathrm{CDCl}_{3}\right)$ of $\mathbf{3 4}$

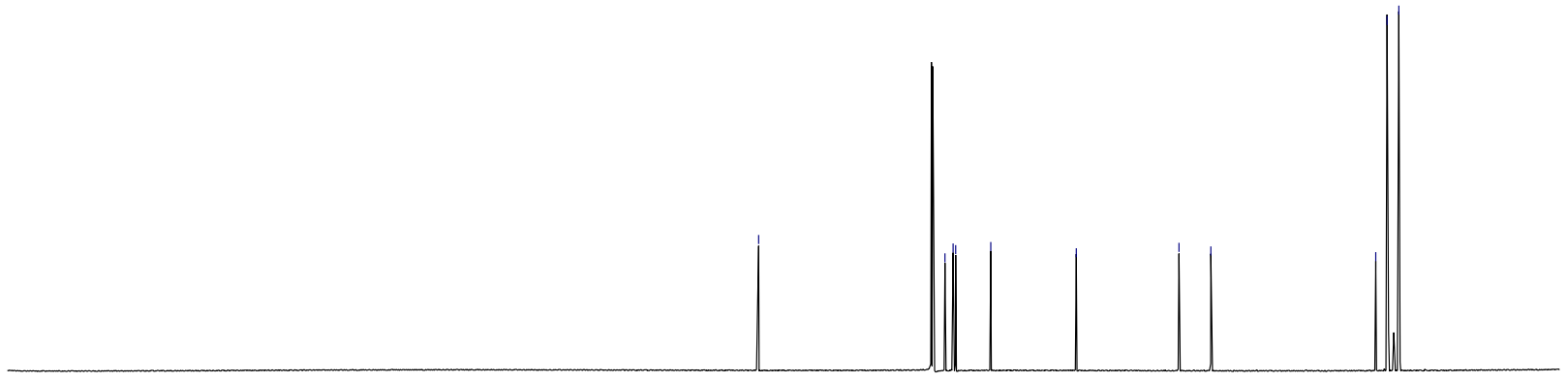

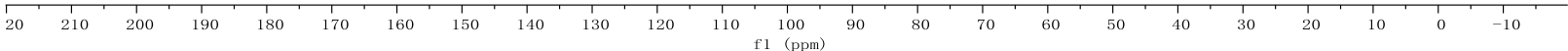


${ }^{1} \mathrm{H}$ NMR $\left(600 \mathrm{MHz}, \mathrm{C}_{6} \mathrm{D}_{6}\right)$ of $\mathbf{3 5}$
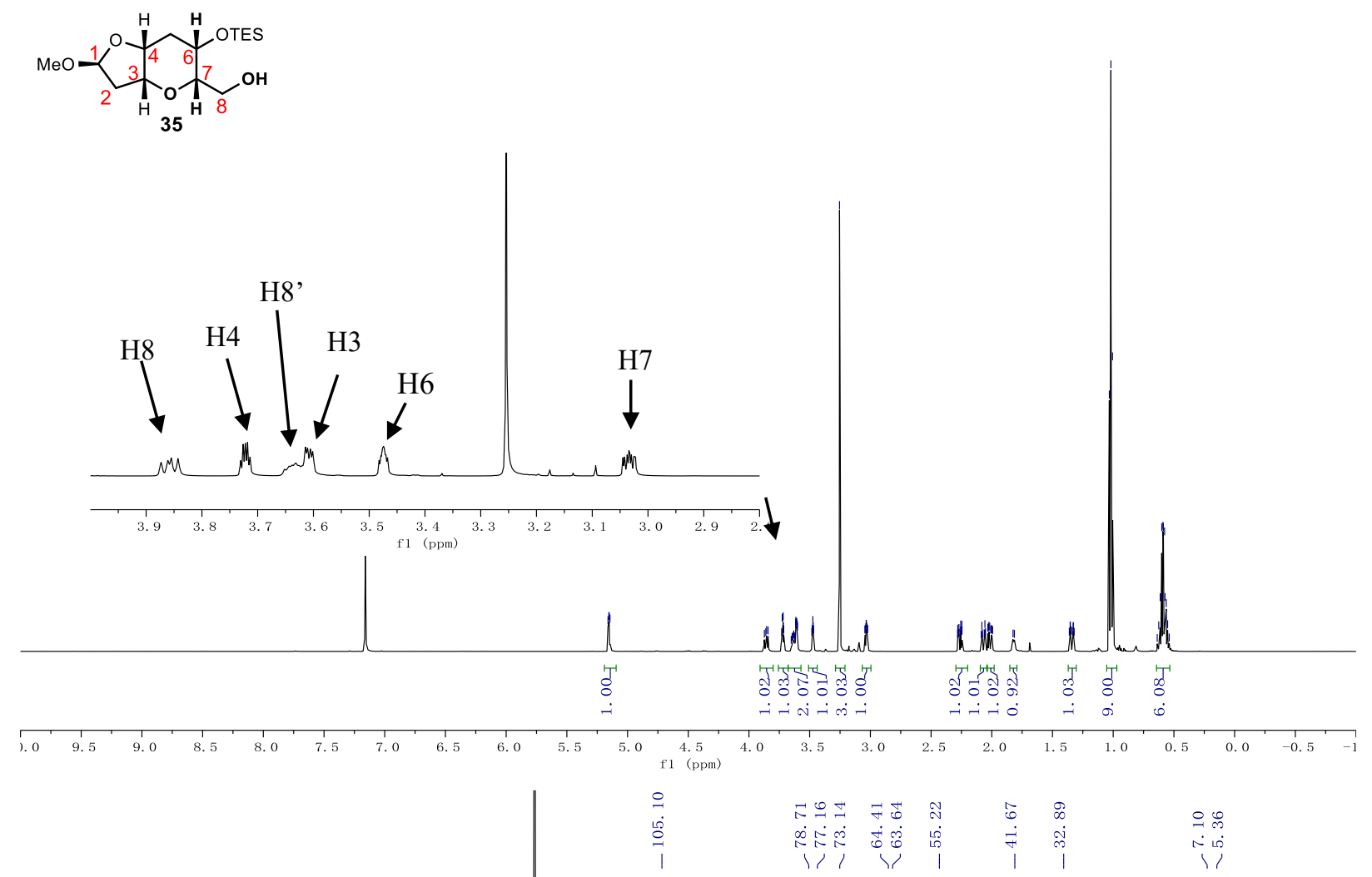

${ }^{13} \mathrm{C}$ NMR $\left(151 \mathrm{MHz}, \mathrm{C}_{6} \mathrm{D}_{6}\right)$ of $\mathbf{3 5}$
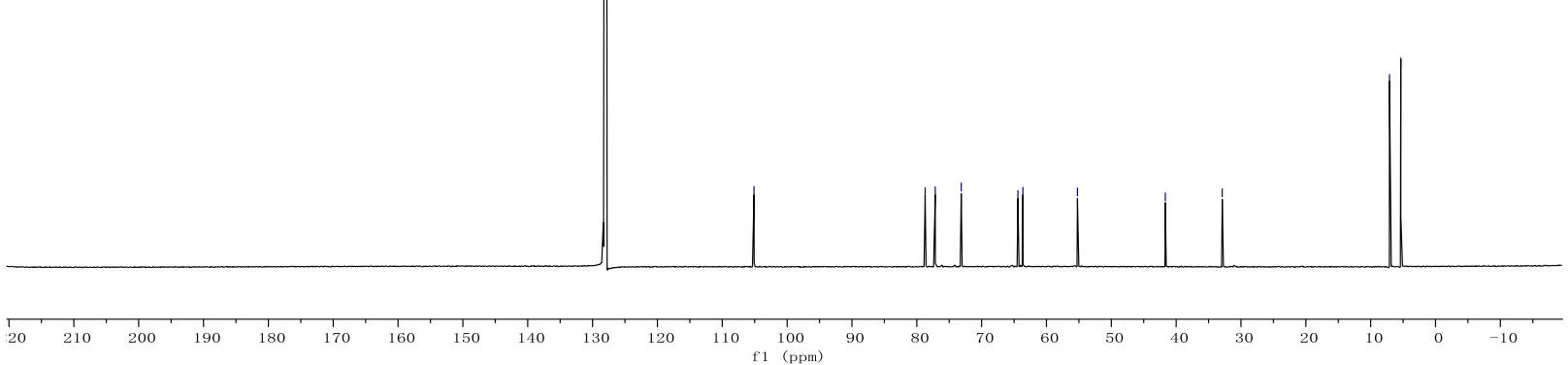

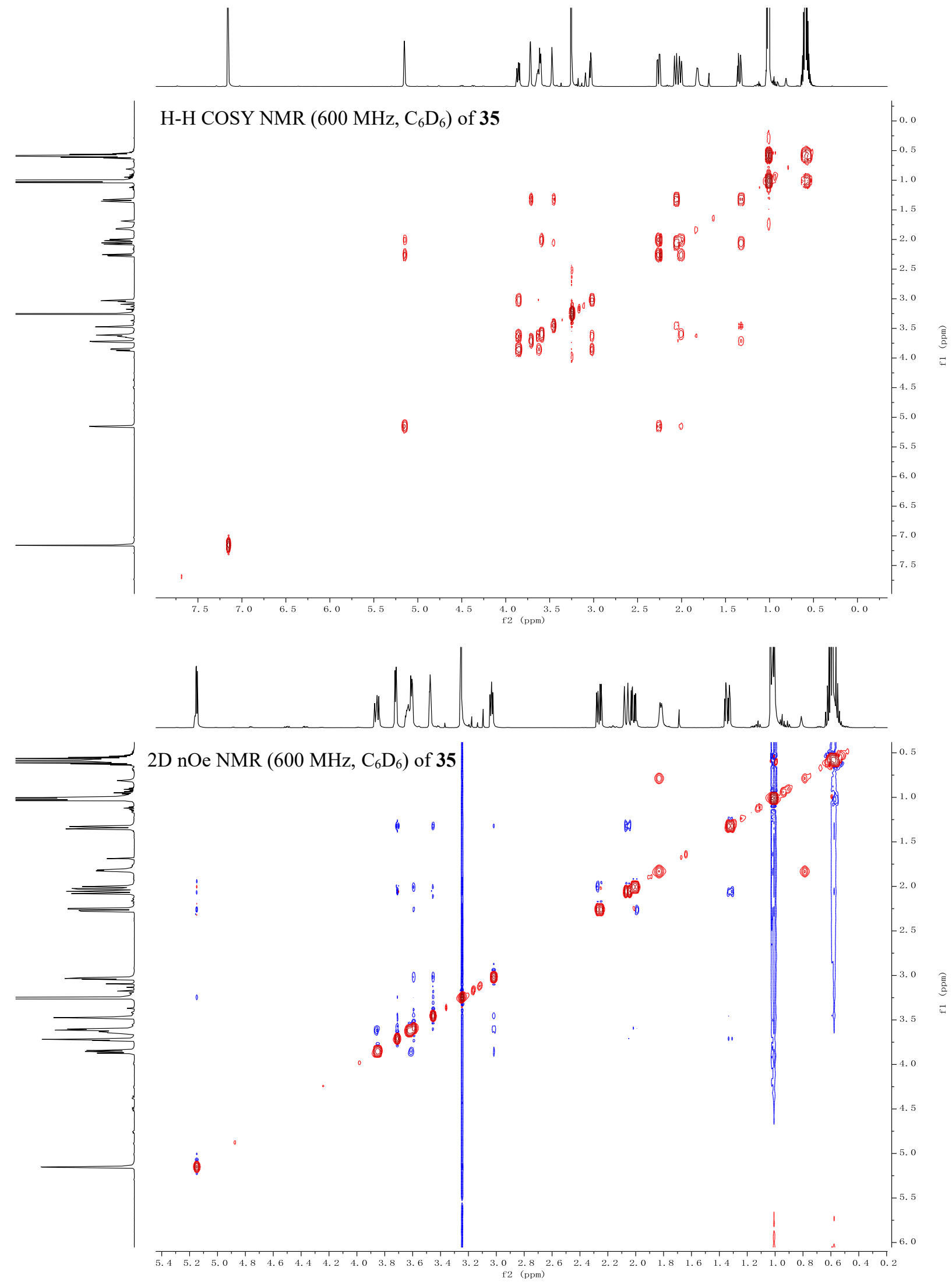
${ }^{1} \mathrm{H}$ NMR $\left(600 \mathrm{MHz}, \mathrm{C}_{6} \mathrm{D}_{6}\right)$ of $\mathbf{3 6}$
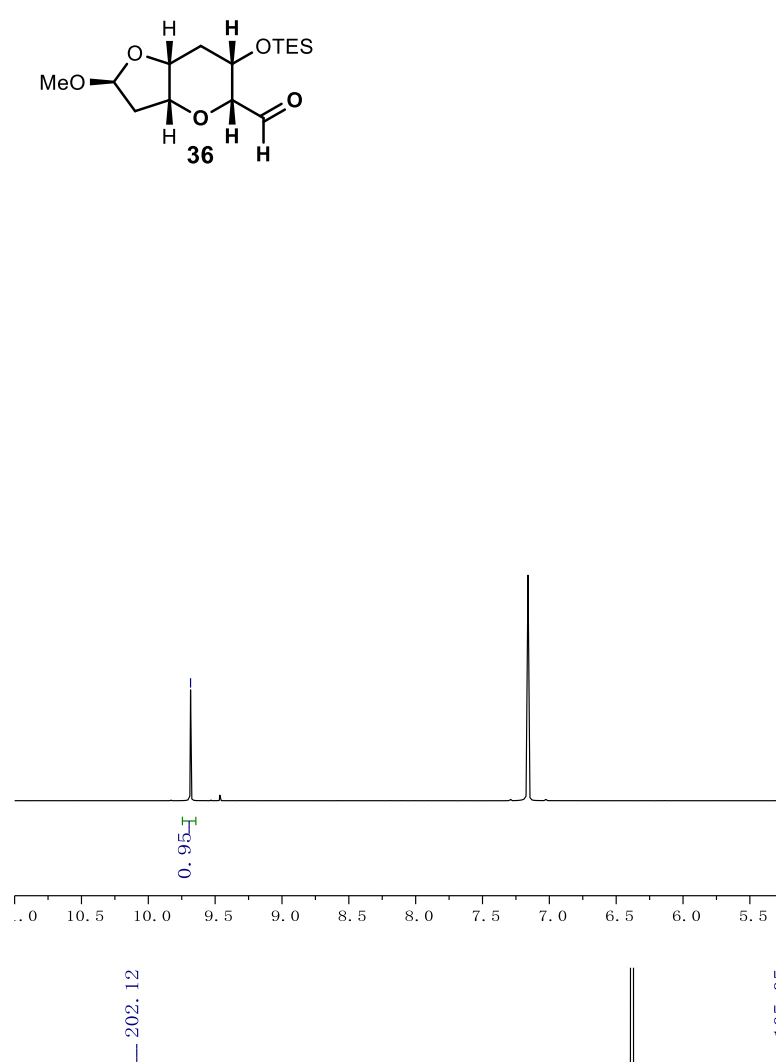

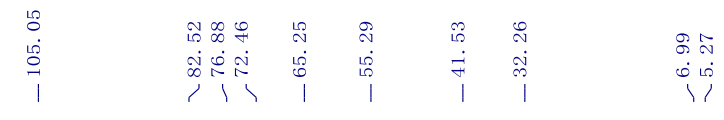

${ }^{13} \mathrm{C}$ NMR $\left(151 \mathrm{MHz}, \mathrm{C}_{6} \mathrm{D}_{6}\right)$ of $\mathbf{3 6}$
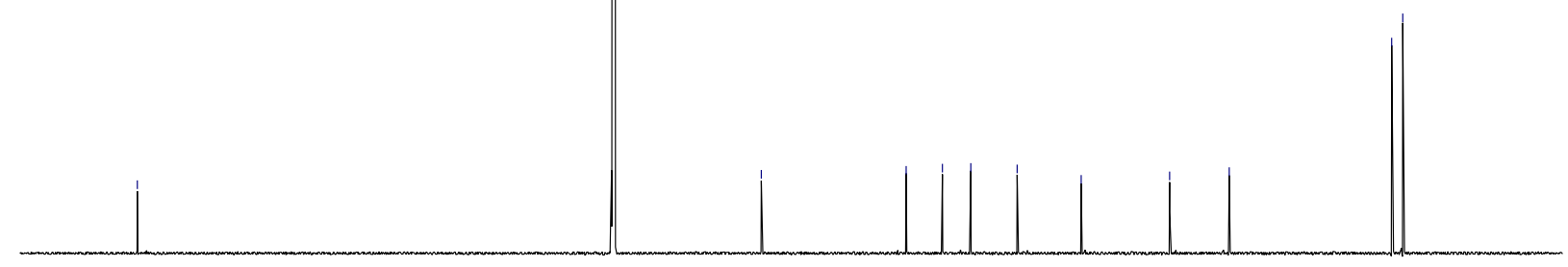

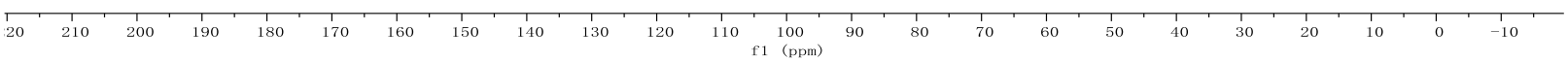


${ }^{1} \mathrm{H}$ NMR $\left(600 \mathrm{MHz}, \mathrm{CD}_{3} \mathrm{CN}\right)$ of $\mathbf{3 7 b}$
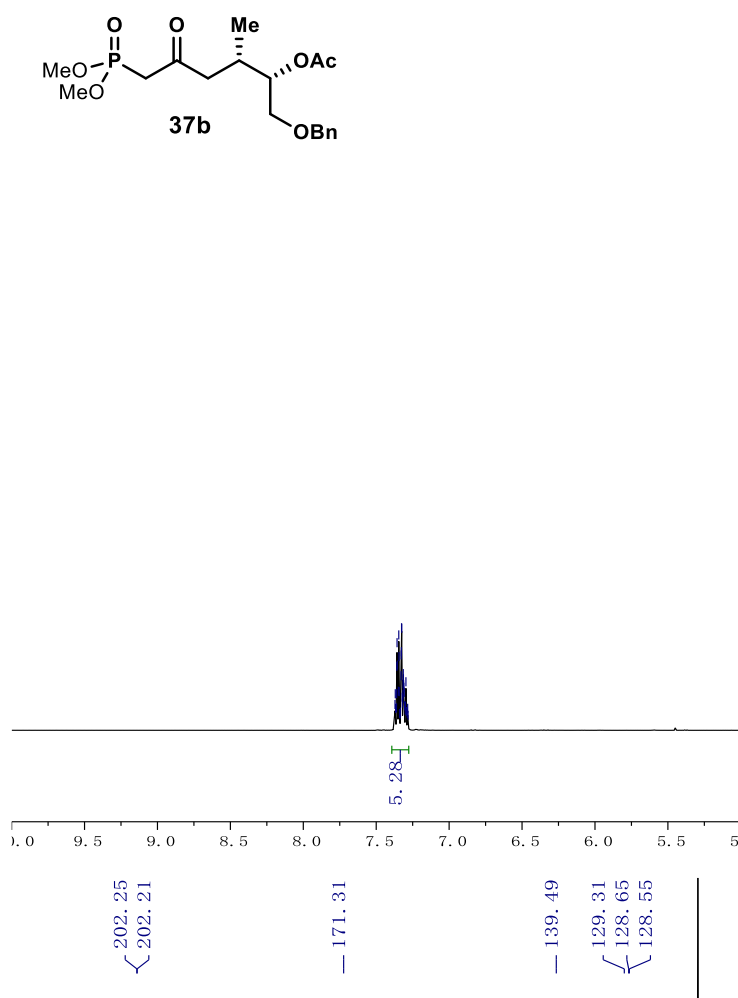

${ }^{13} \mathrm{C}$ NMR $\left(151 \mathrm{MHz}, \mathrm{CD}_{3} \mathrm{CN}\right)$ of $\mathbf{3 7 b}$

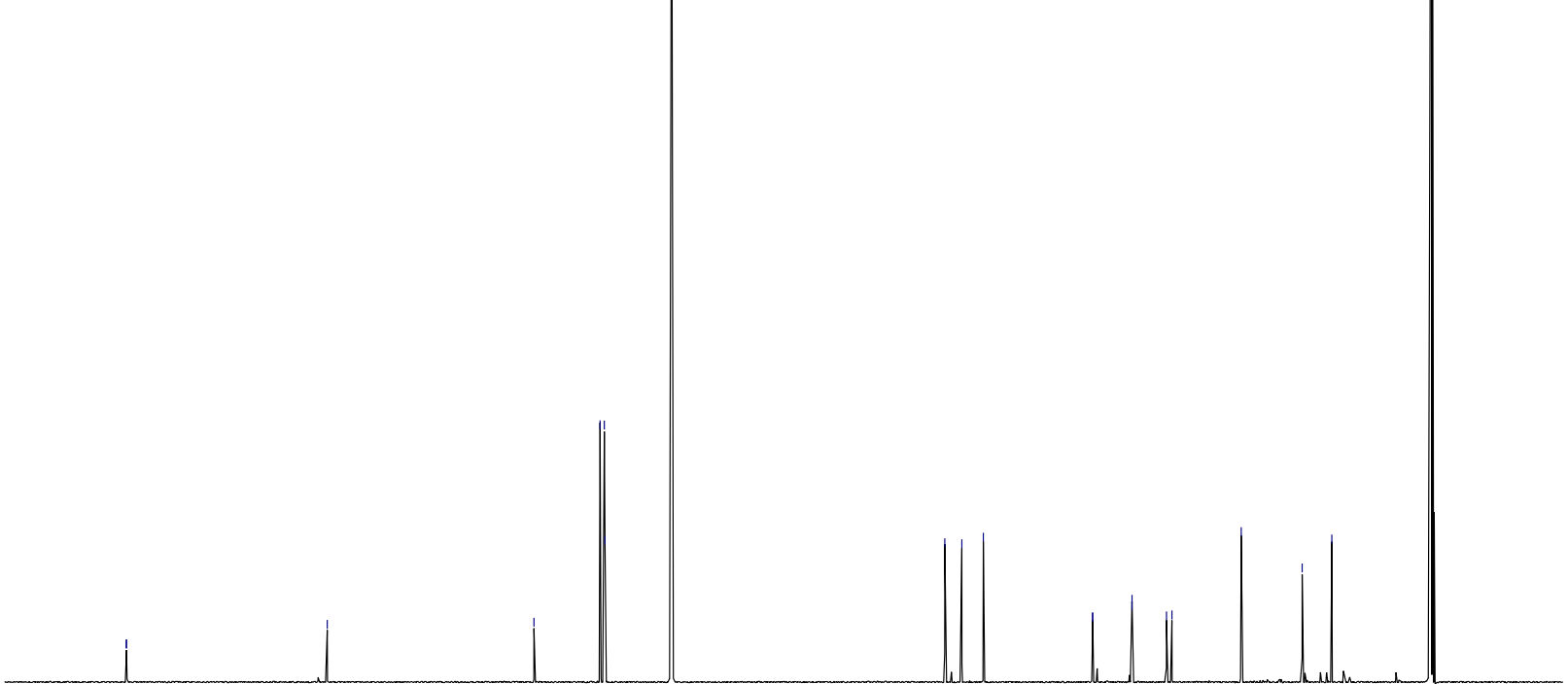

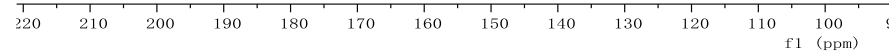


${ }^{1} \mathrm{H}$ NMR $\left(600 \mathrm{MHz}, \mathrm{C}_{6} \mathrm{D}_{6}\right)$ of $\mathbf{3 8 a}$

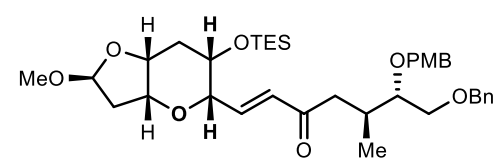

$38 \mathbf{a}$

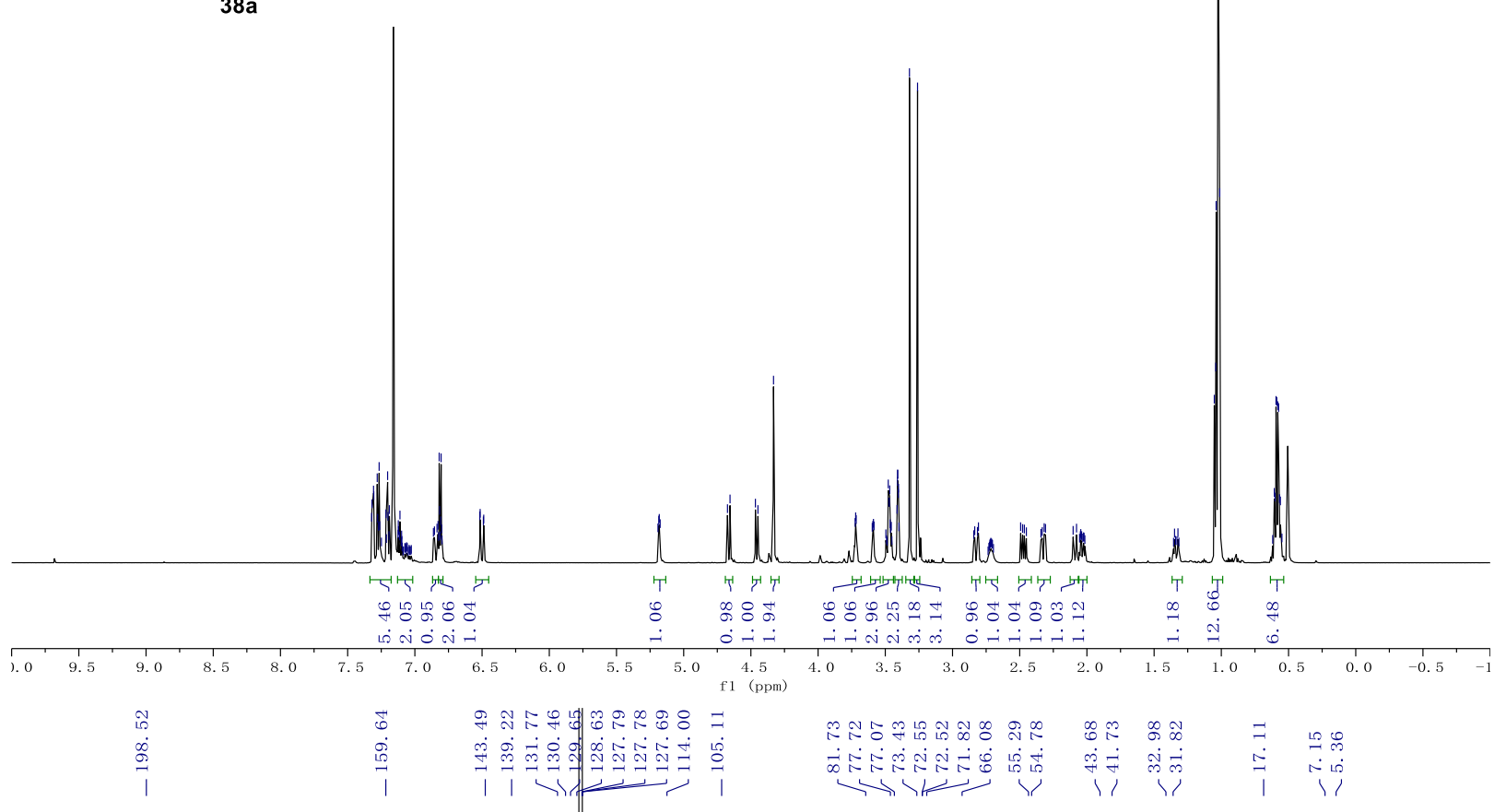

${ }^{13} \mathrm{C}$ NMR $\left(151 \mathrm{MHz}, \mathrm{C}_{6} \mathrm{D}_{6}\right)$ of $\mathbf{3 8 a}$
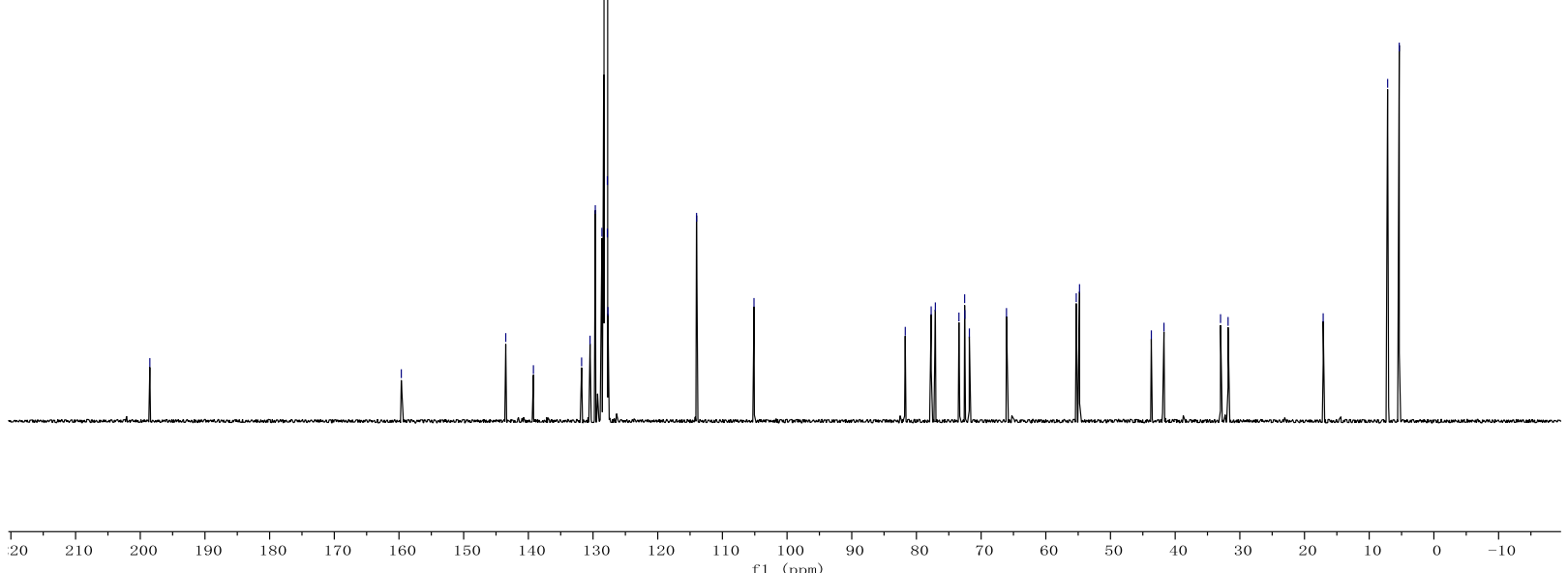
${ }^{1} \mathrm{H}$ NMR $\left(600 \mathrm{MHz}, \mathrm{C}_{6} \mathrm{D}_{6}\right)$ of $\mathbf{3 8 b}$
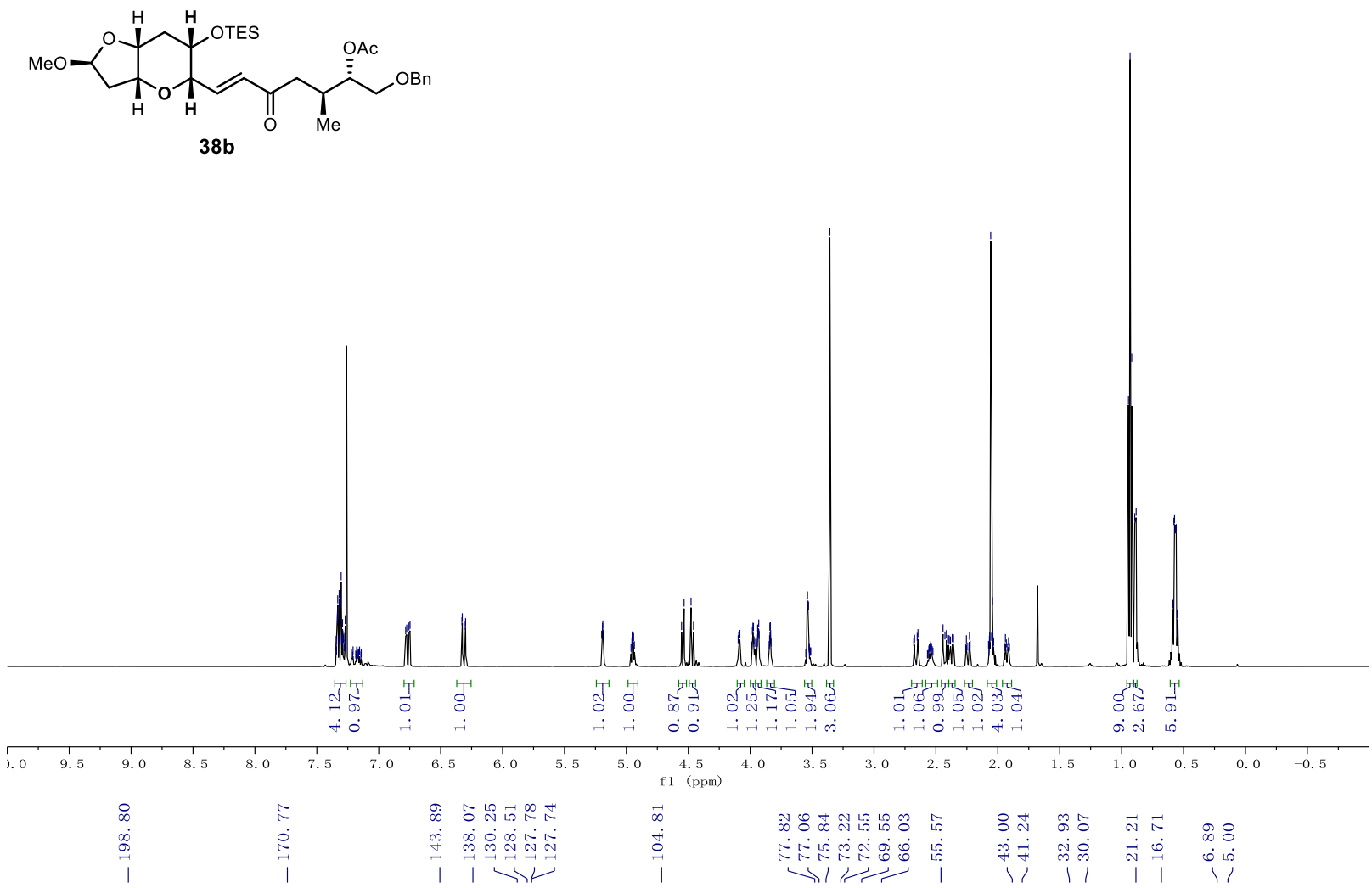

${ }^{13} \mathrm{C}$ NMR $\left(151 \mathrm{MHz}, \mathrm{C}_{6} \mathrm{D}_{6}\right)$ of $\mathbf{3 8 b}$

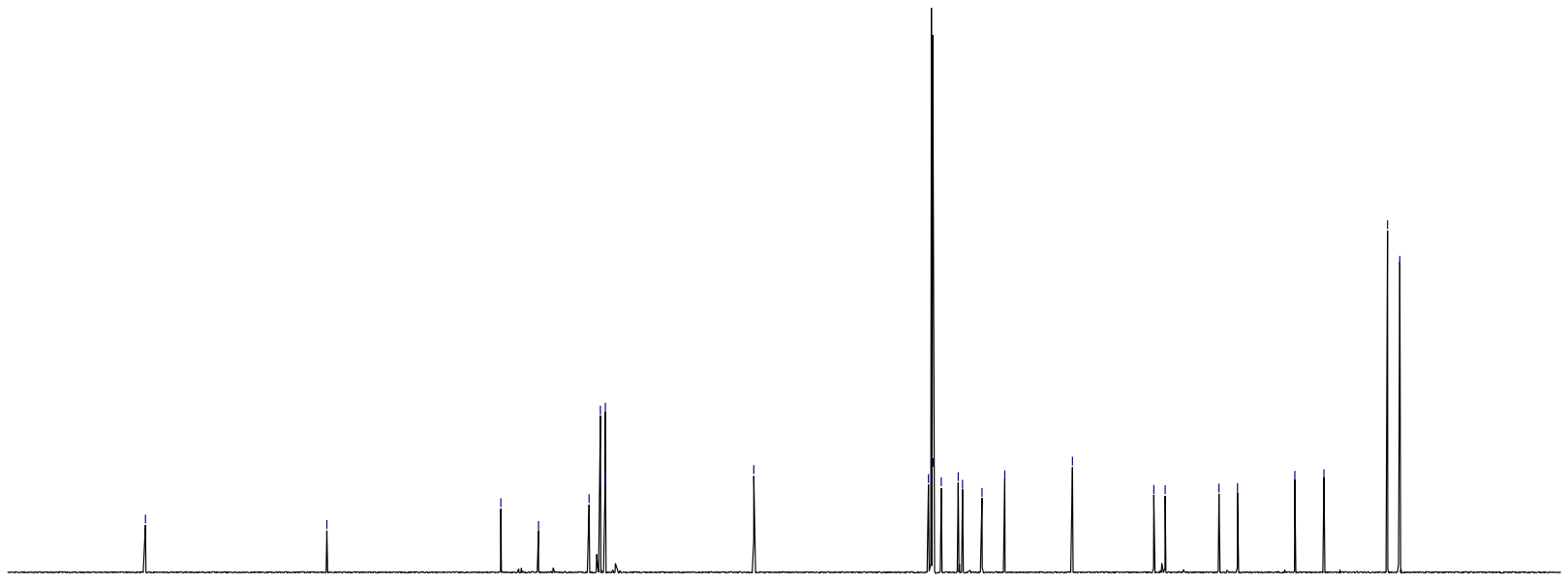

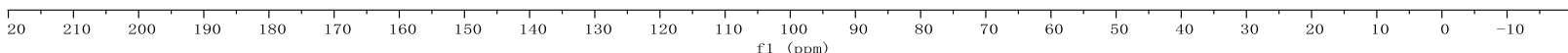


${ }^{1} \mathrm{H}$ NMR $\left(600 \mathrm{MHz}, \mathrm{C}_{6} \mathrm{D}_{6}\right)$ of 39a

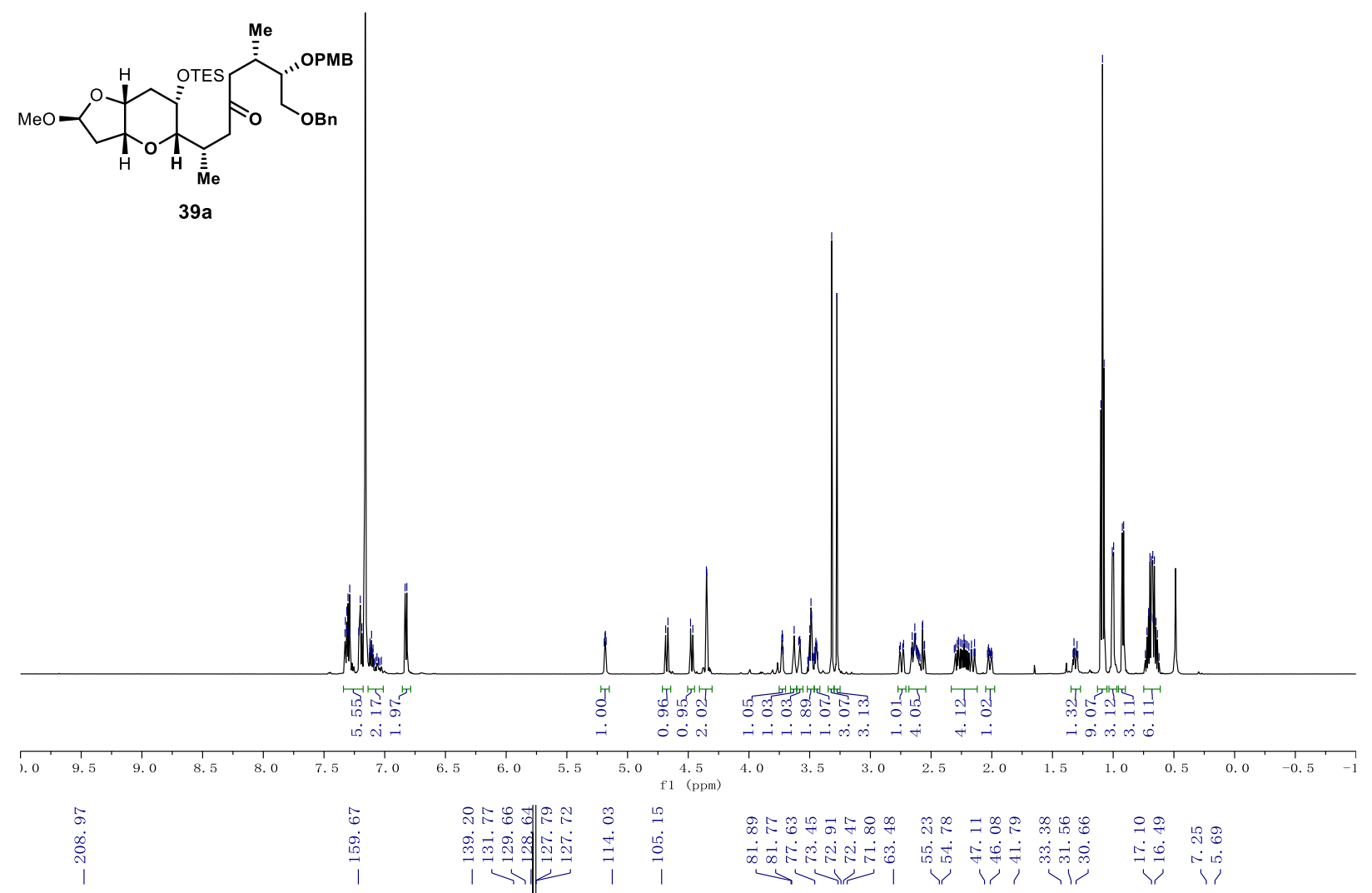

${ }^{13} \mathrm{C}$ NMR (151 MHz, $\left.\mathrm{C}_{6} \mathrm{D}_{6}\right)$ of 39a
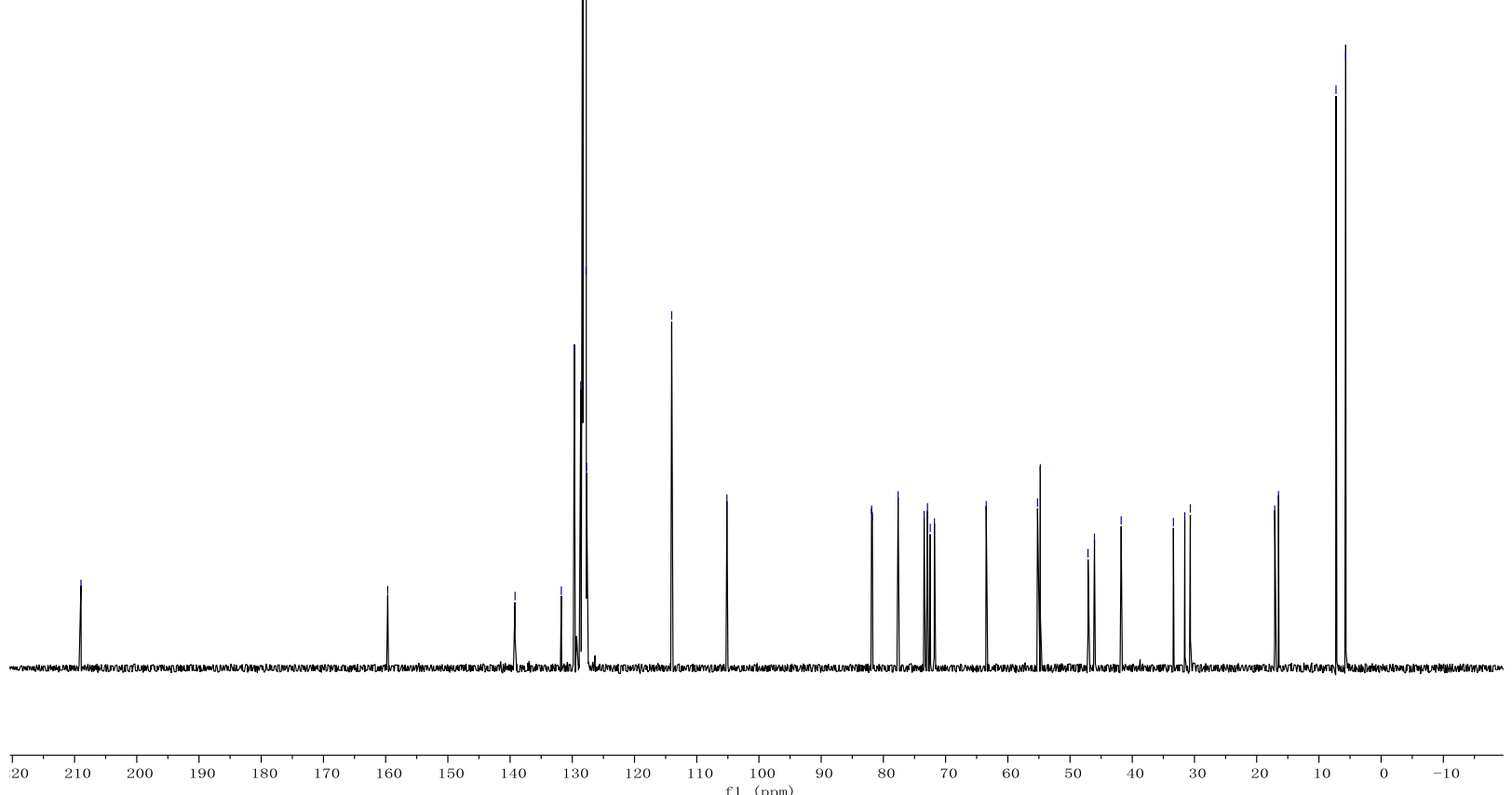
${ }^{1} \mathrm{H}$ NMR $\left(600 \mathrm{MHz}, \mathrm{CDCl}_{3}\right)$ of $\mathbf{3 9 b}$

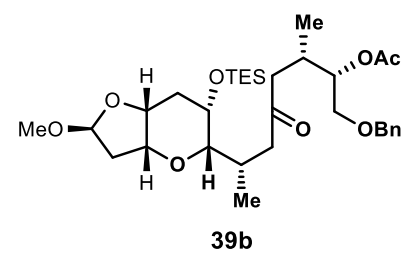

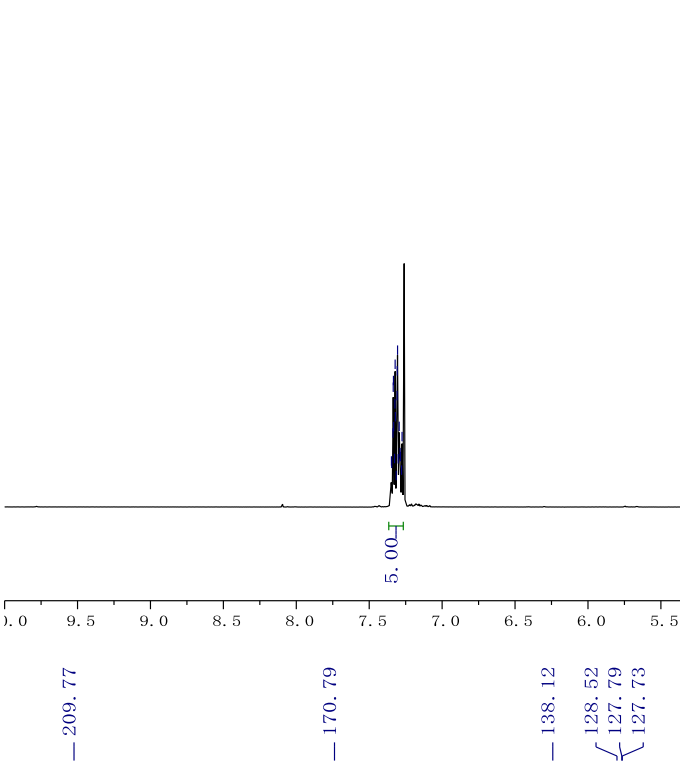

${ }^{13} \mathrm{C} \mathrm{NMR}\left(151 \mathrm{MHz}, \mathrm{CDCl}_{3}\right)$ of $\mathbf{3 9 b}$ - - i

$\stackrel{+}{\infty}$

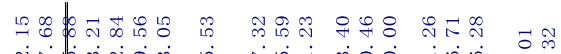

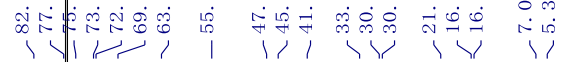

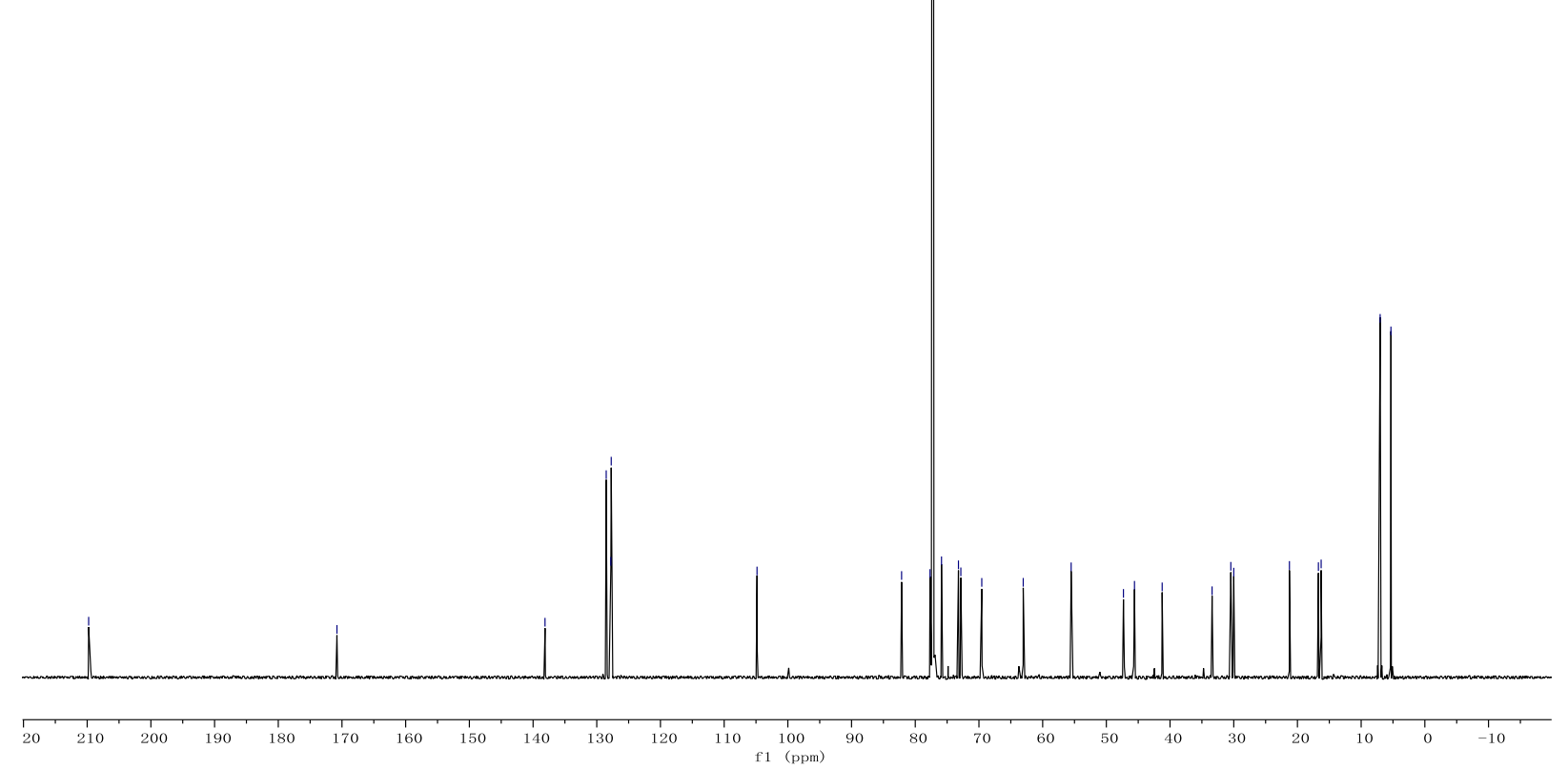


${ }^{1} \mathrm{H}$ NMR $\left(600 \mathrm{MHz}, \mathrm{C}_{6} \mathrm{D}_{6}\right)$ of $\mathbf{3 2 a}$

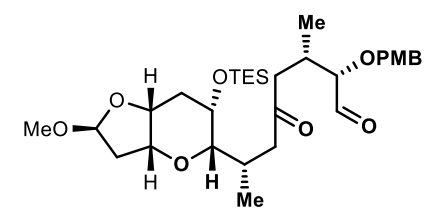

32a: fragment $A$
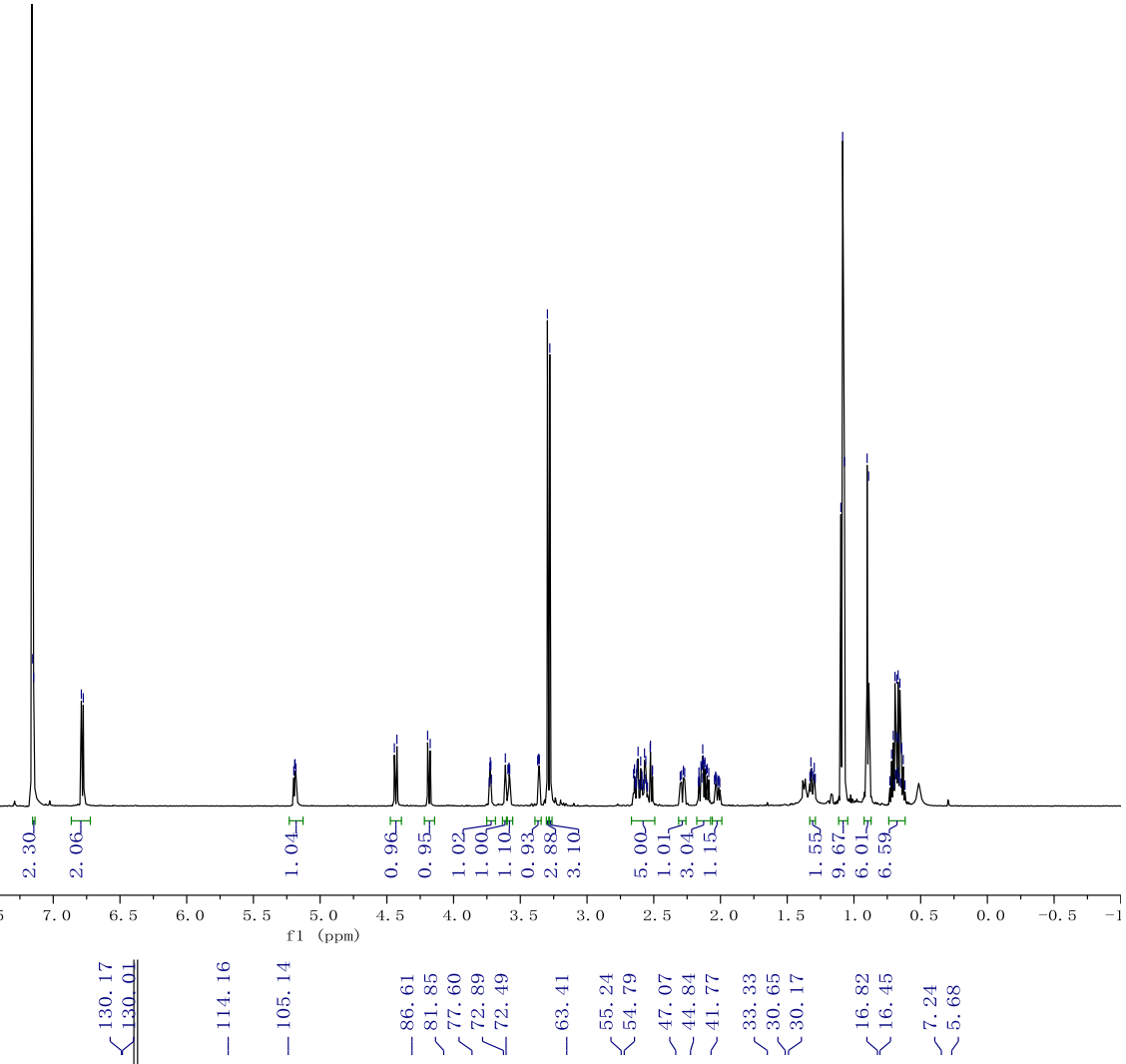

${ }^{13} \mathrm{C}$ NMR $\left(151 \mathrm{MHz}, \mathrm{C}_{6} \mathrm{D}_{6}\right)$ of $\mathbf{3 2} \mathbf{a}$
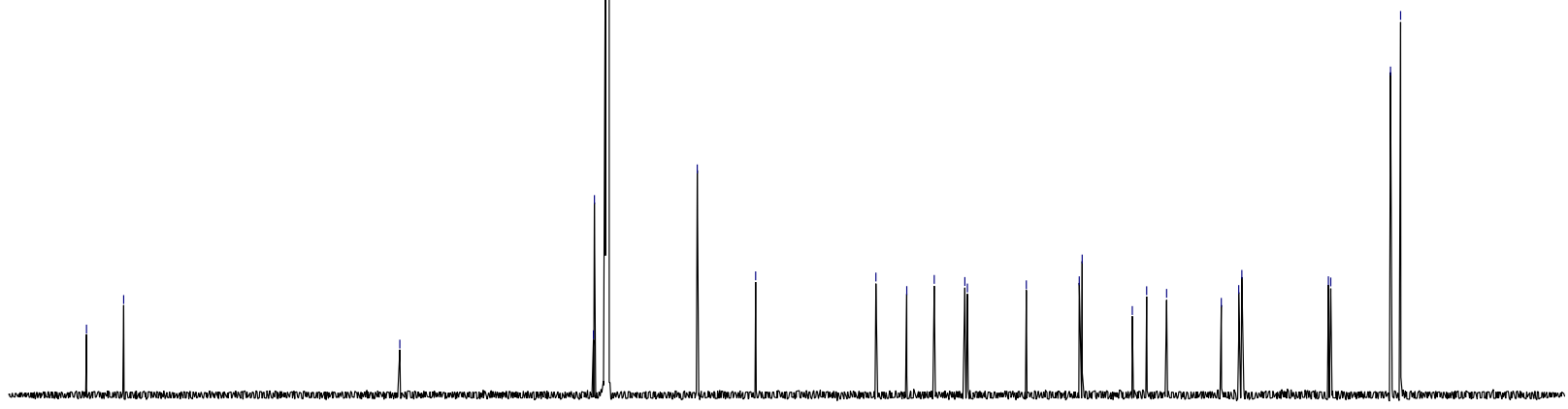

20

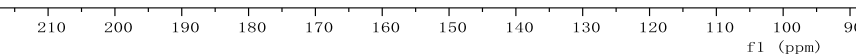


${ }^{1} \mathrm{H}$ NMR $\left(600 \mathrm{MHz}, \mathrm{C}_{6} \mathrm{D}_{6}\right)$ of $\mathbf{3 2 b}$<smiles>CO[C@H](C=O)[C@@H](C)C(=O)C[C@@H](C)[C@H](C)CC(=O)O</smiles>

32b: fragment $A^{\prime}$

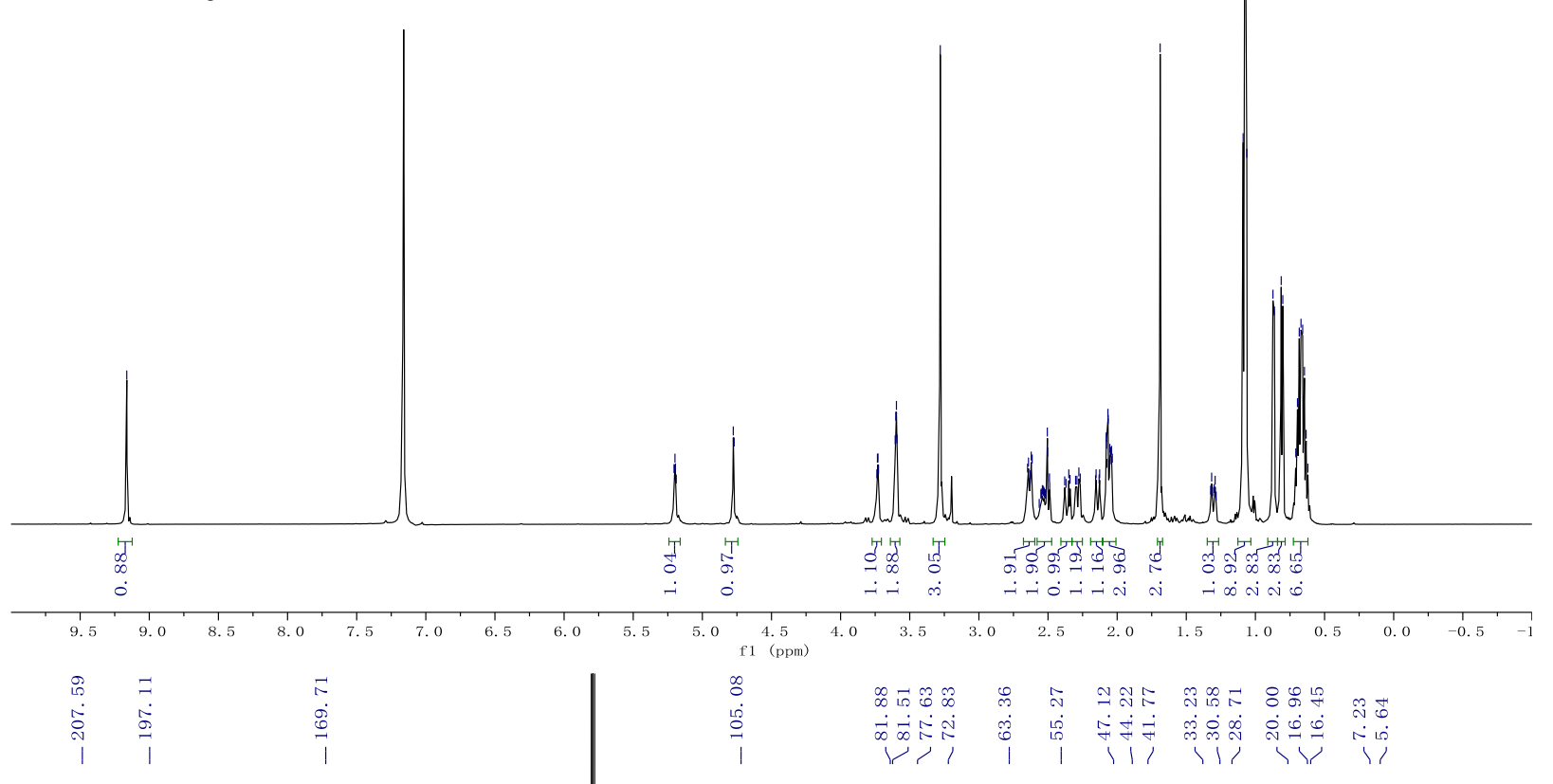

${ }^{13} \mathrm{C}$ NMR (151 MHz, $\left.\mathrm{C}_{6} \mathrm{D}_{6}\right)$ of $\mathbf{3 2 b}$
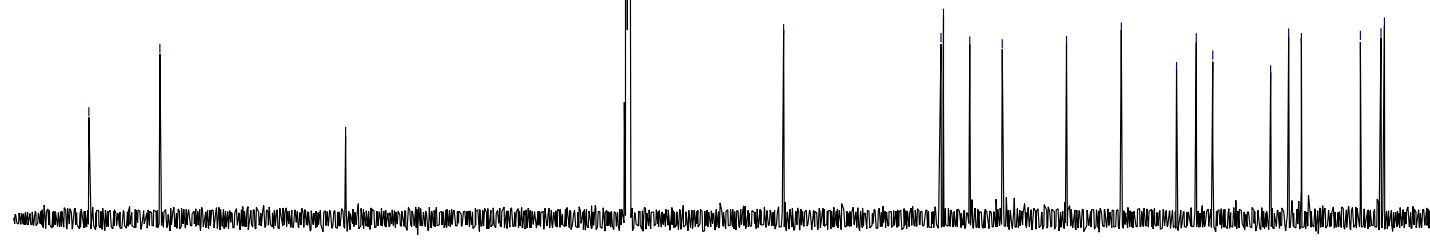
${ }^{1} \mathrm{H} \mathrm{NMR}\left(600 \mathrm{MHz}, \mathrm{CDCl}_{3}\right)$ of $\mathbf{4 0}$
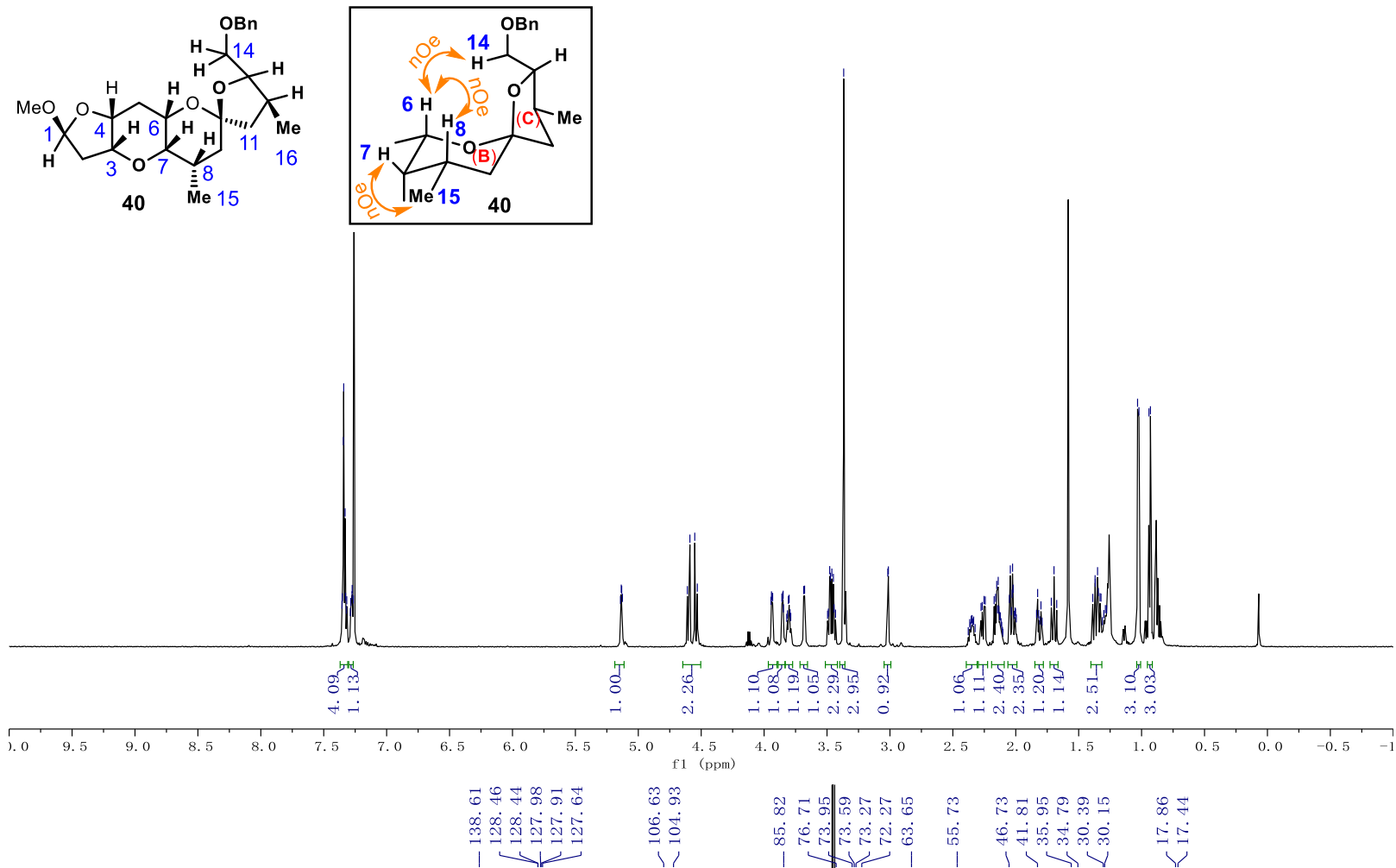

$$
\begin{aligned}
& m 8 \\
& 0 \% \\
& 80 \\
& 01
\end{aligned}
$$

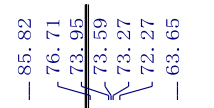

${ }^{13} \mathrm{C} \mathrm{NMR}\left(151 \mathrm{MHz}, \mathrm{CDCl}_{3}\right)$ of $\mathbf{4 0}$

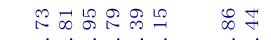

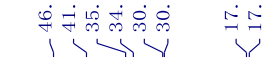
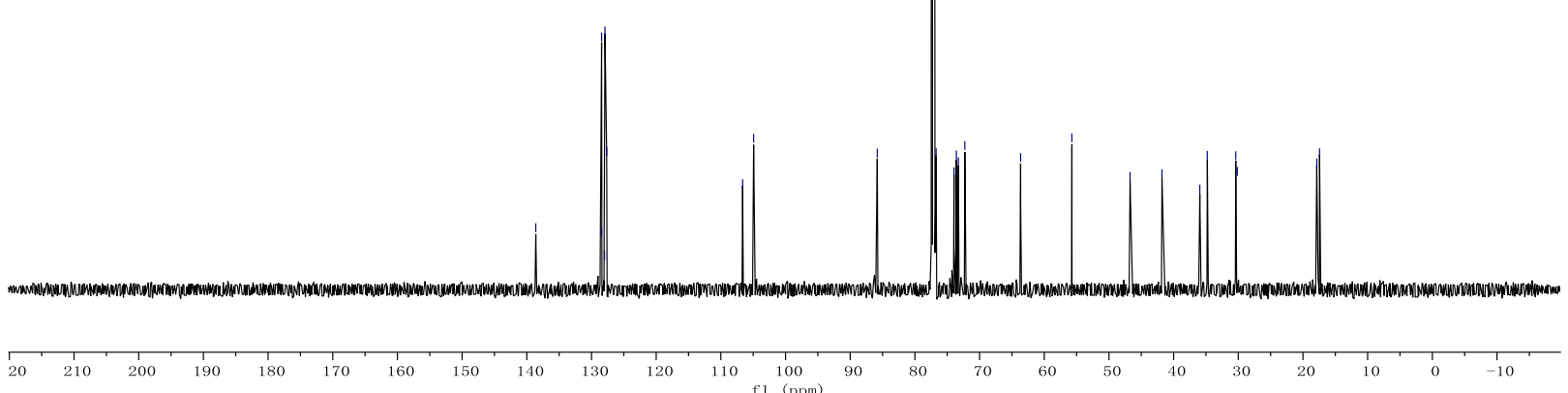


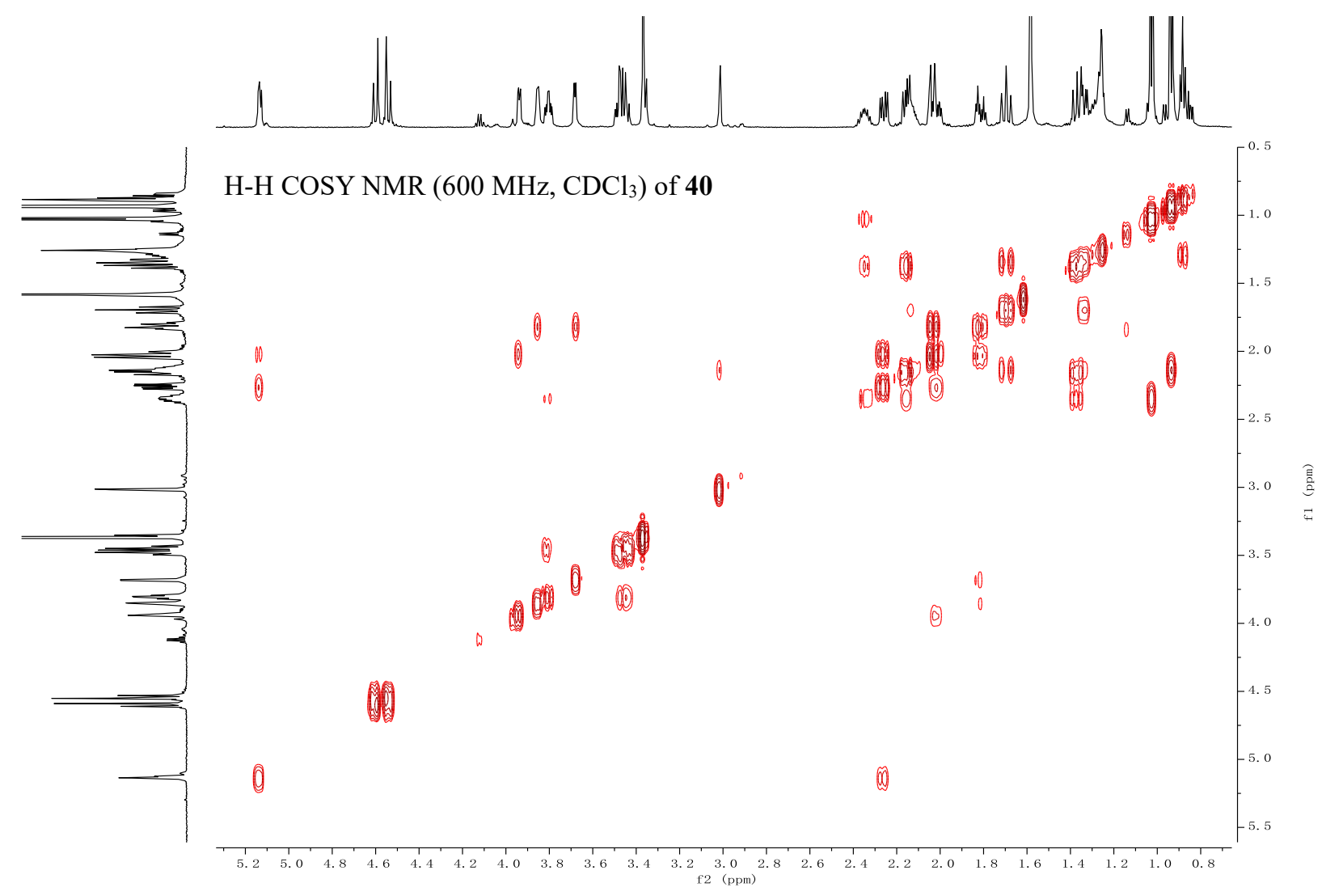

1D Selective Gradient NOESY (600 MHz, $\left.\mathrm{CDCl}_{3}\right)$ of $\mathbf{4 0}$
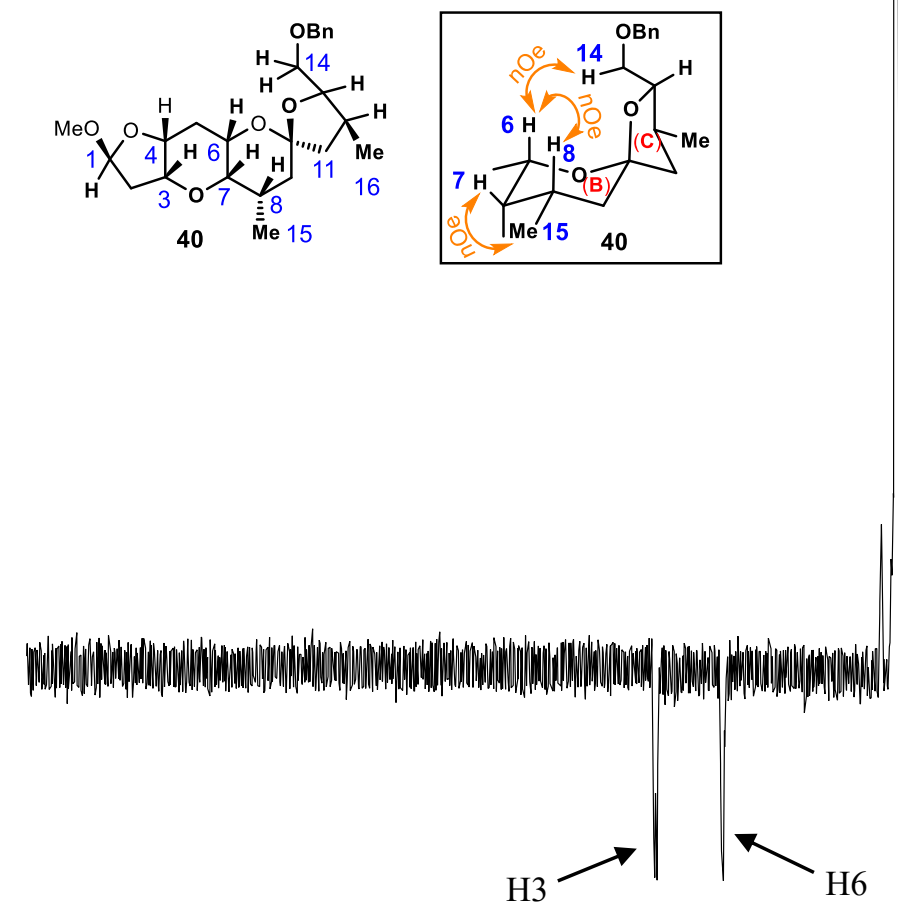

Irradiated H7

\begin{tabular}{llllllllllllllllllllll}
\hline 6.2 & 6.0 & 5.8 & 5.6 & 5.4 & 5.2 & 5.0 & 4.8 & 4.6 & 4.4 & 4.2 & 4.0 & 3.8 & 3.6 & 3.4 & 3.2 \\
f1 (pm)
\end{tabular} 
1D Selective Gradient NOESY $\left(600 \mathrm{MHz}, \mathrm{CDCl}_{3}\right)$ of $\mathbf{4 0}$

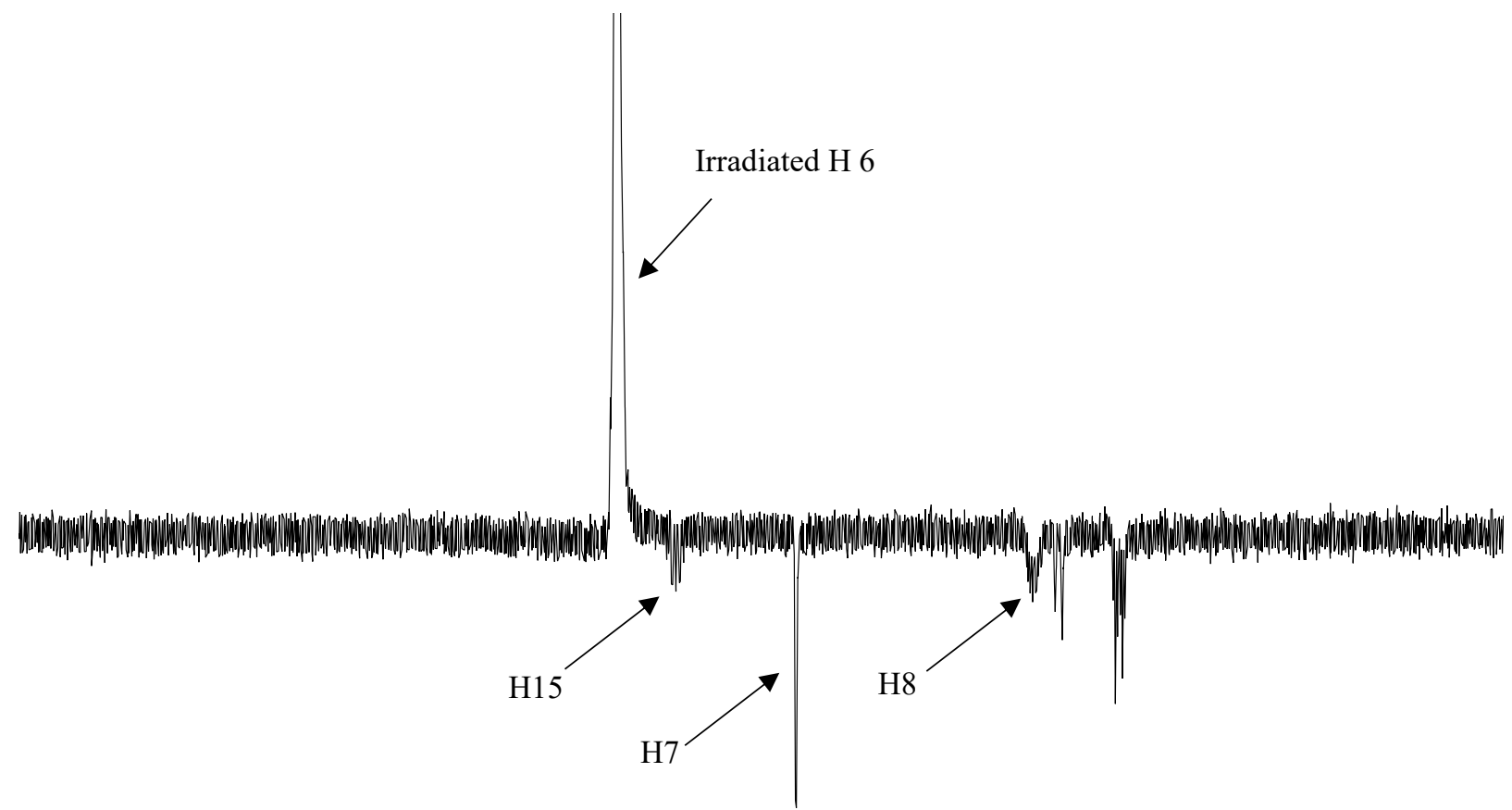

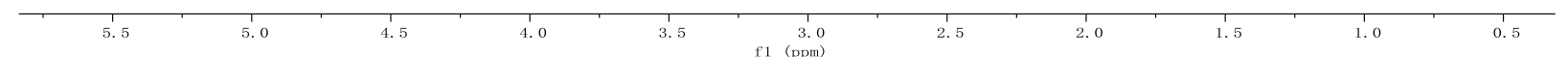

1D Selective Gradient NOESY $\left(600 \mathrm{MHz}, \mathrm{CDCl}_{3}\right)$ of $\mathbf{4 0}$

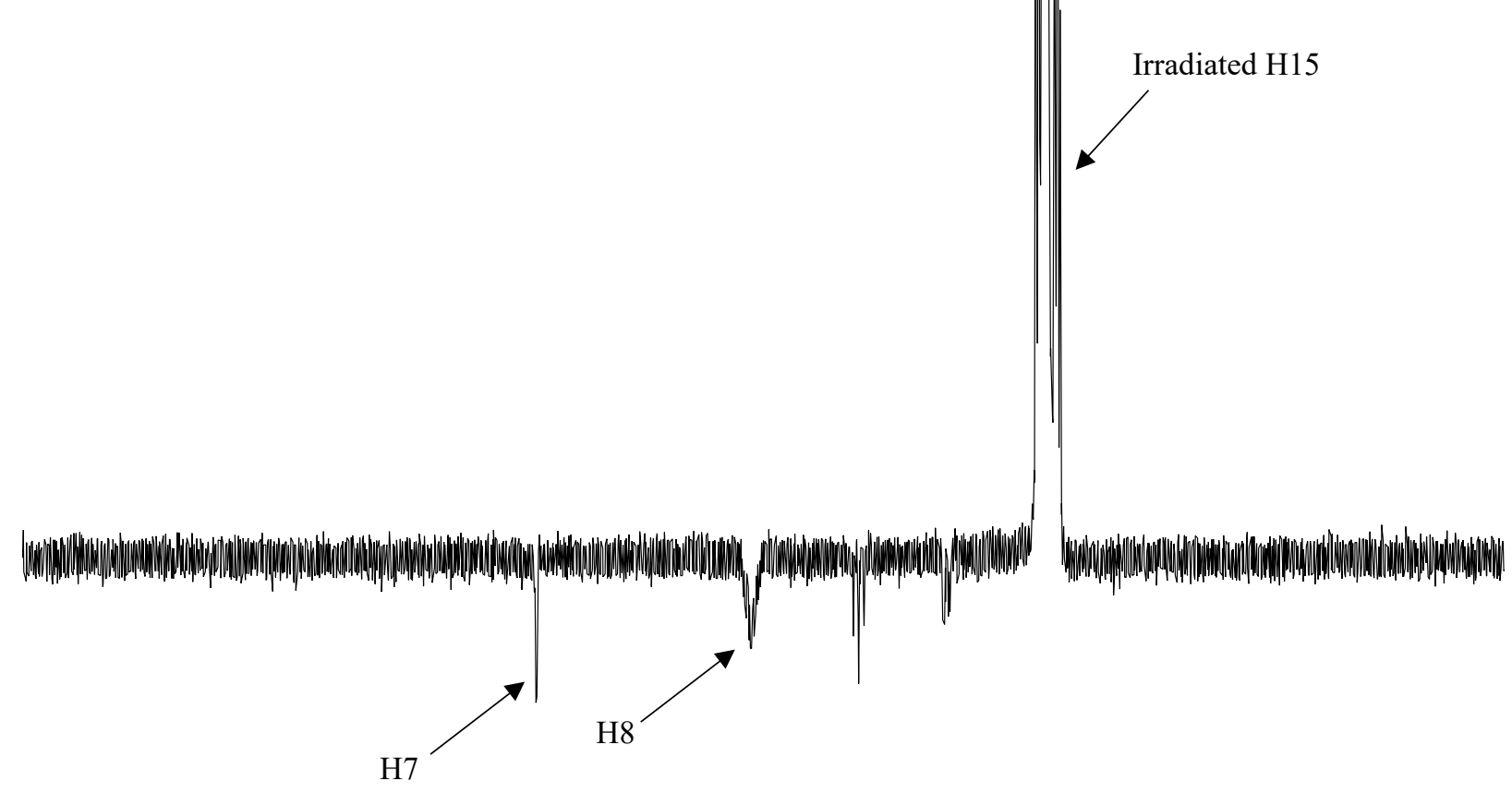

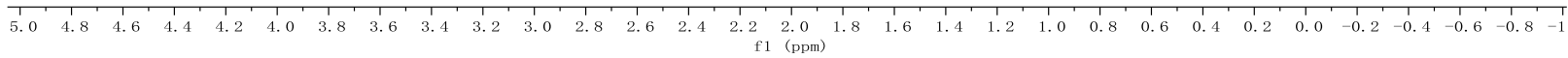


${ }^{1} \mathrm{H}$ NMR (600 MHz, $\mathrm{C}_{6} \mathrm{D}_{6}$ ) of $\mathbf{4 2 / 4 3}$ (mixture of tautomers)

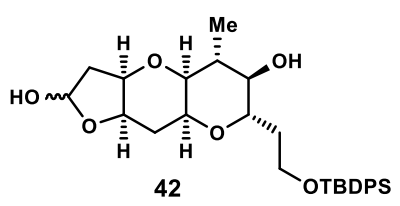

and tautomer $\mathbf{4 3}$

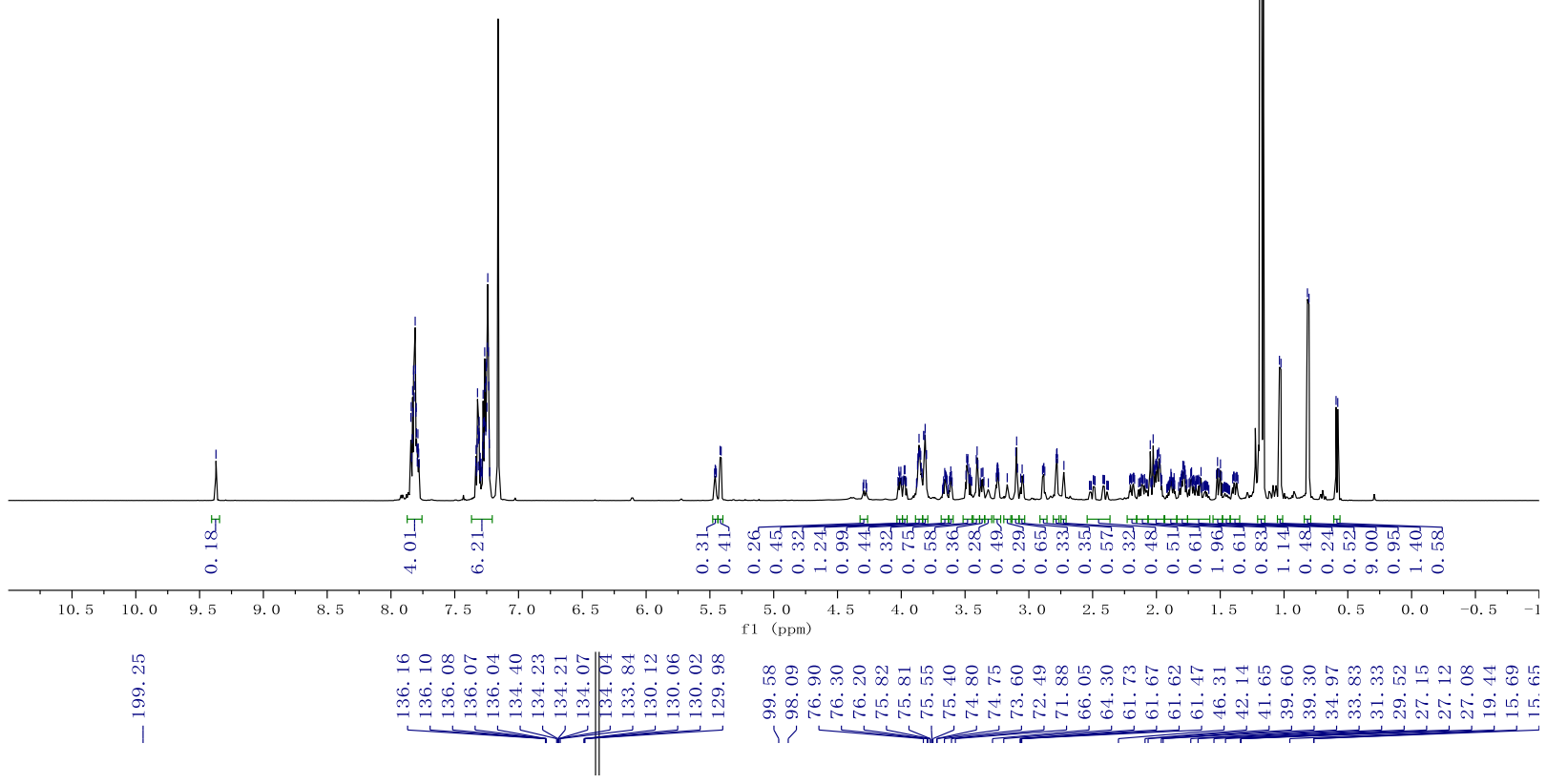

${ }^{13} \mathrm{C}$ NMR (151 MHz, $\mathrm{C}_{6} \mathrm{D}_{6}$ ) of $\mathbf{4 2 / 4 3}$ (mixture of tautomers)
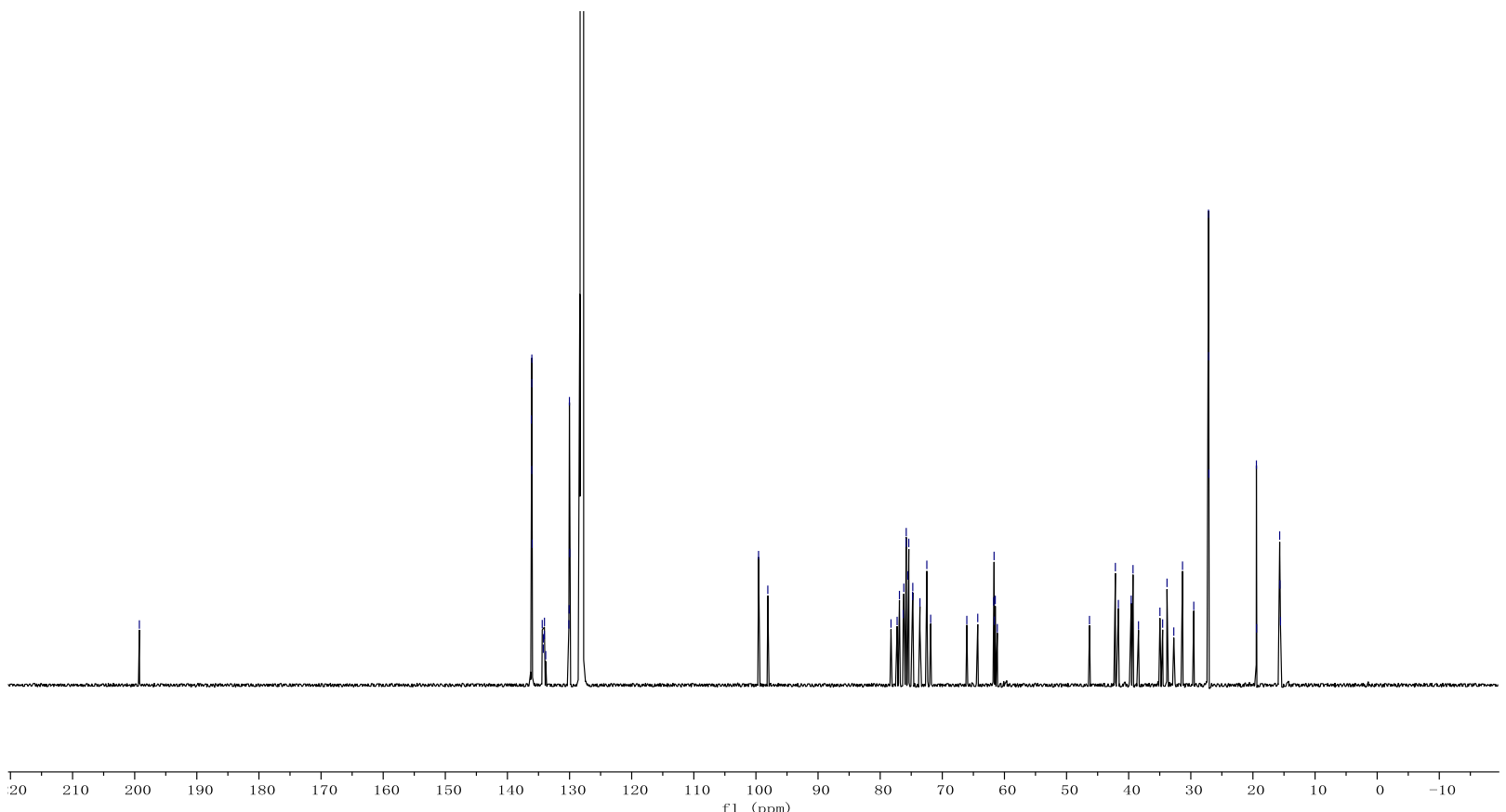
${ }^{1} \mathrm{H}$ NMR (600 MHz, $\left.\mathrm{CD}_{3} \mathrm{CN}\right)$ of 45 (major) + tautomers
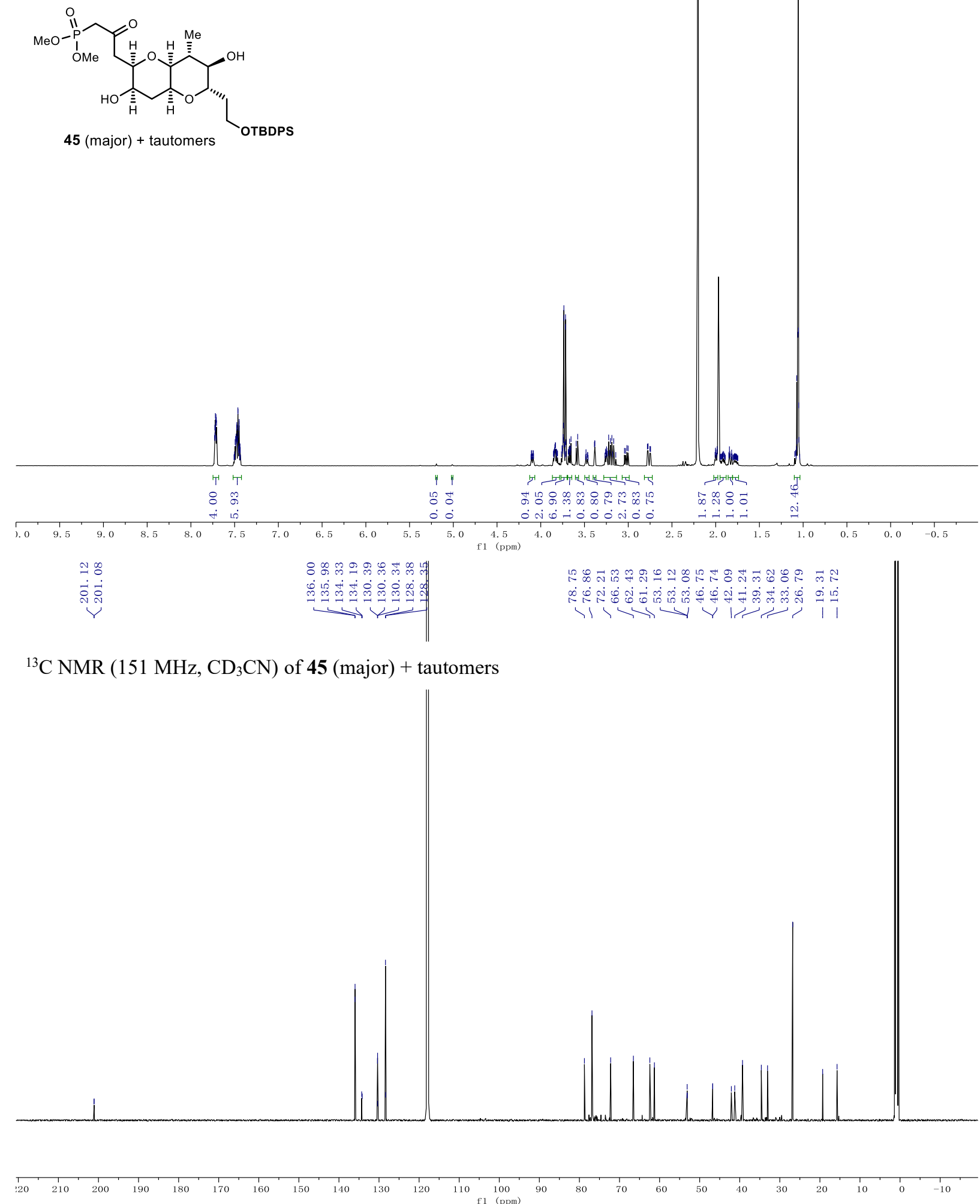
${ }^{1} \mathrm{H}$ NMR $\left(600 \mathrm{MHz}, \mathrm{C}_{6} \mathrm{D}_{6}\right)$ of $\mathbf{4 6 a}$

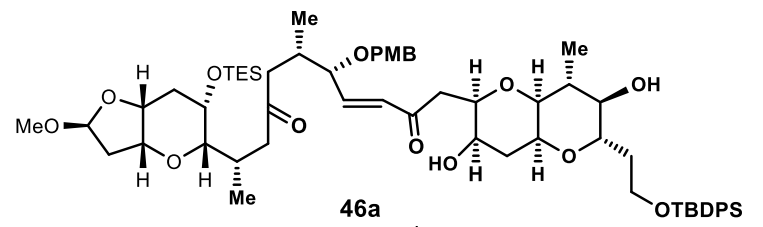
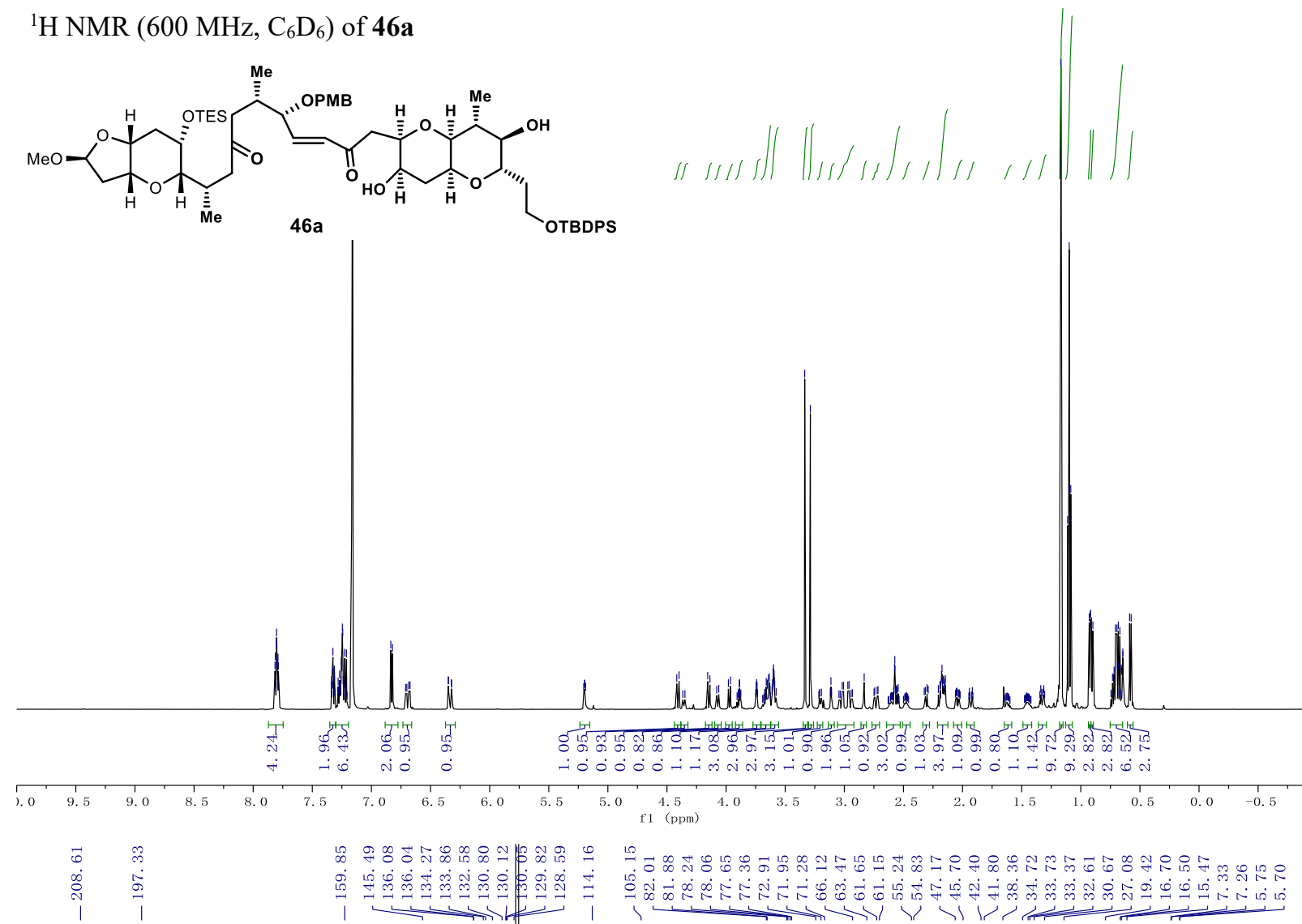

${ }^{13} \mathrm{C}$ NMR (151 MHz, $\left.\mathrm{C}_{6} \mathrm{D}_{6}\right)$ of $\mathbf{4 6 a}$

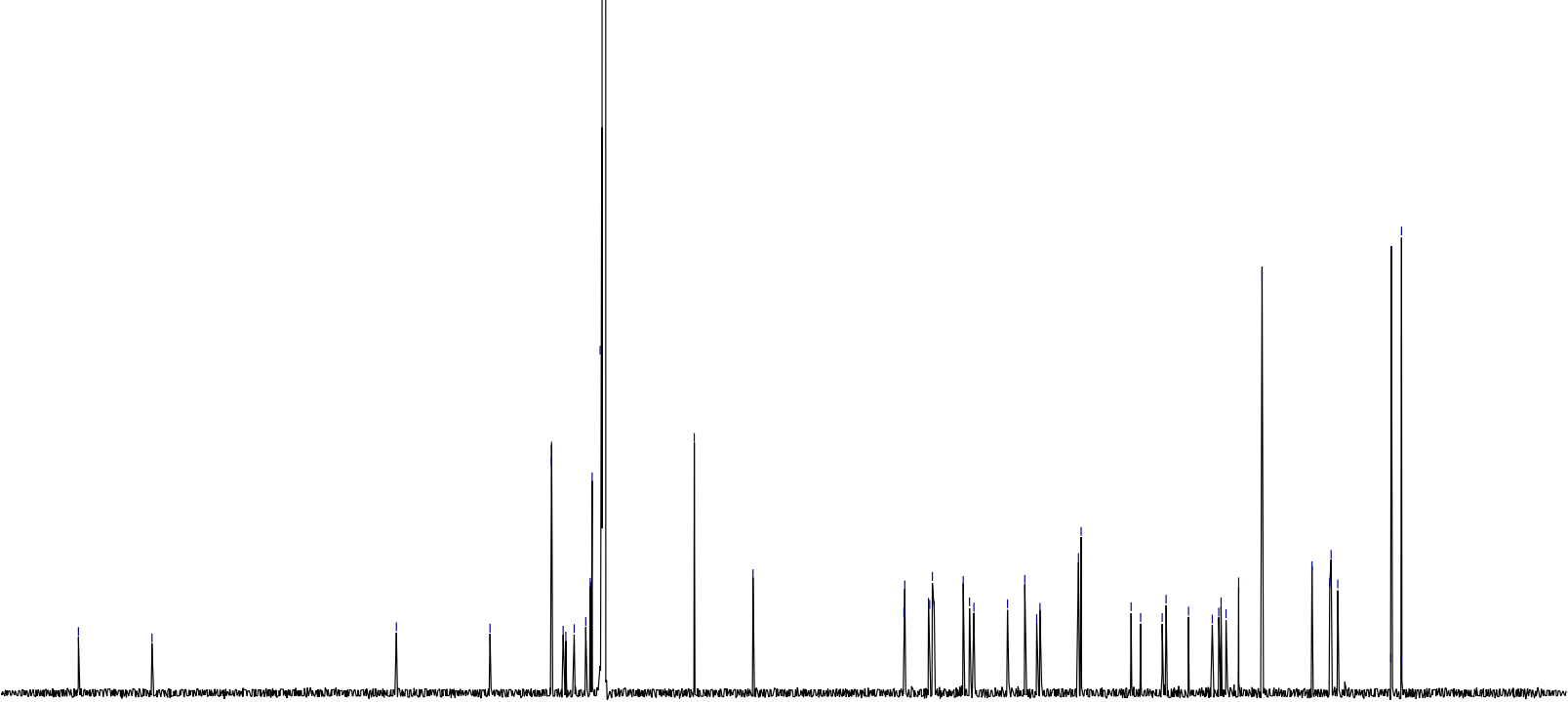

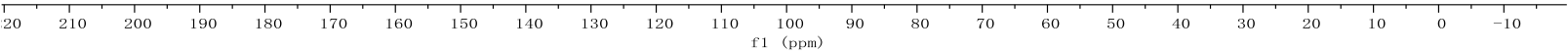



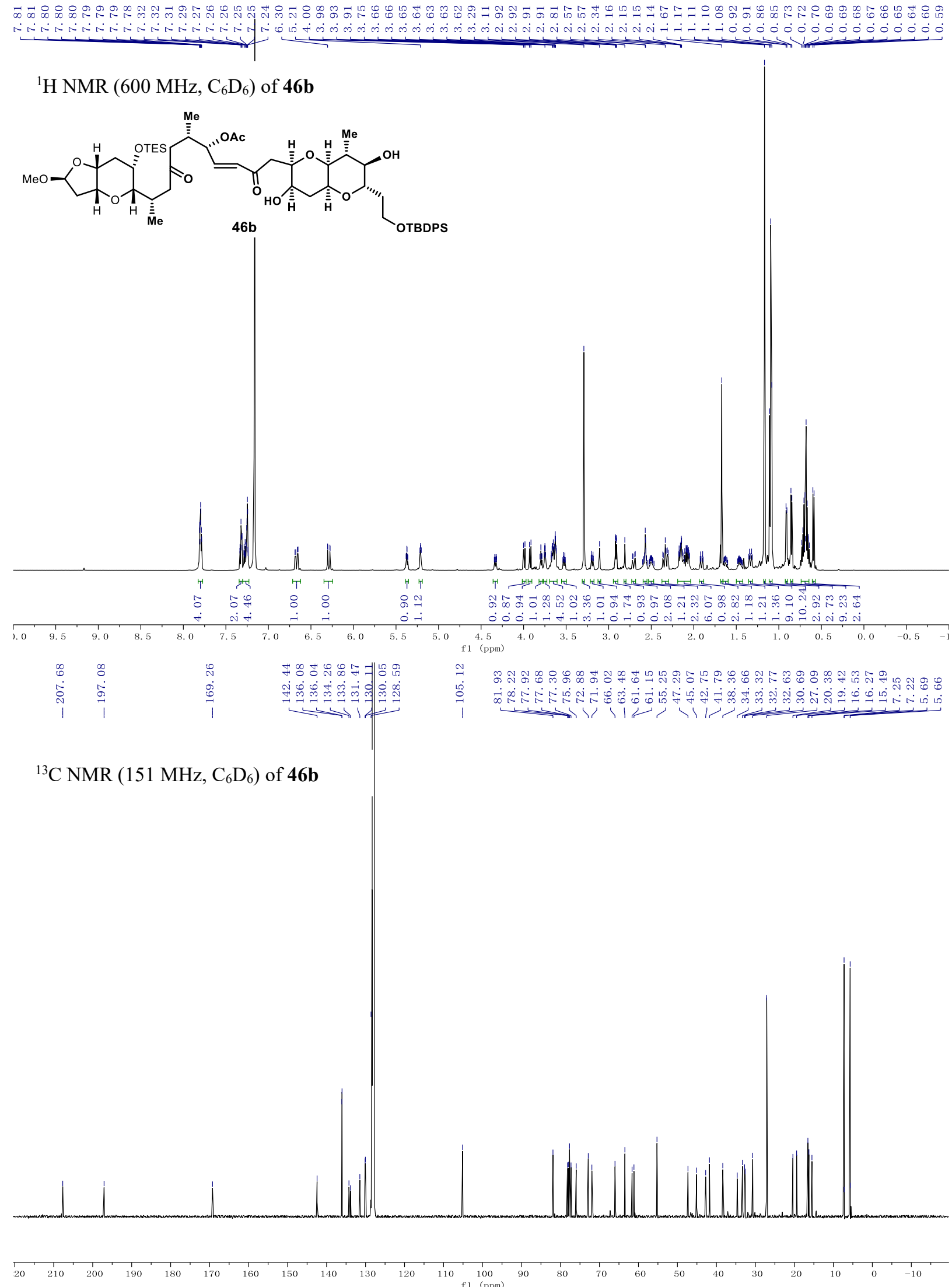
${ }^{1} \mathrm{H}$ NMR $\left(600 \mathrm{MHz}, \mathrm{C}_{6} \mathrm{D}_{6}\right)$ of 47

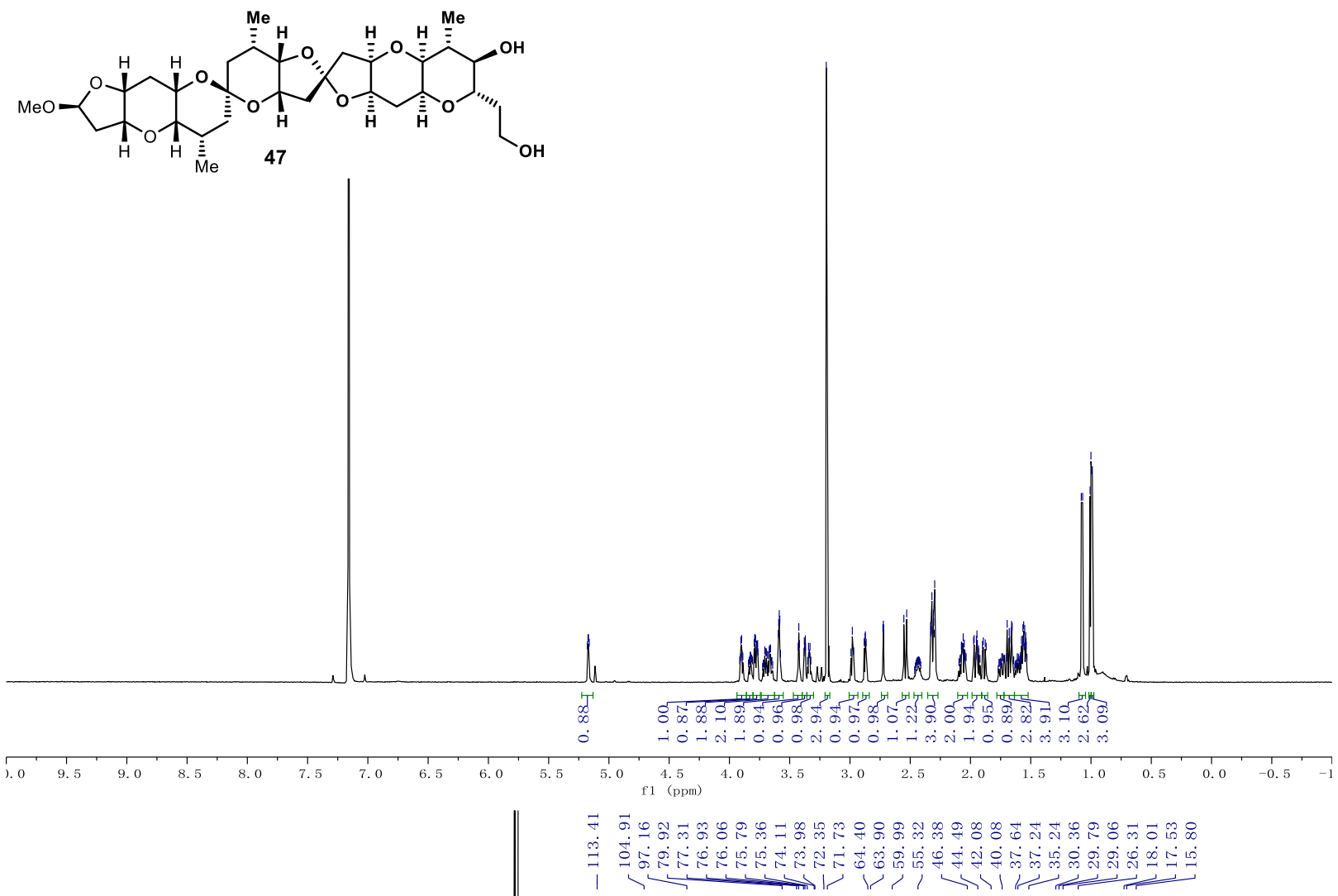

${ }^{13} \mathrm{C}$ NMR (151 MHz, $\left.\mathrm{C}_{6} \mathrm{D}_{6}\right)$ of $\mathbf{4 7}$
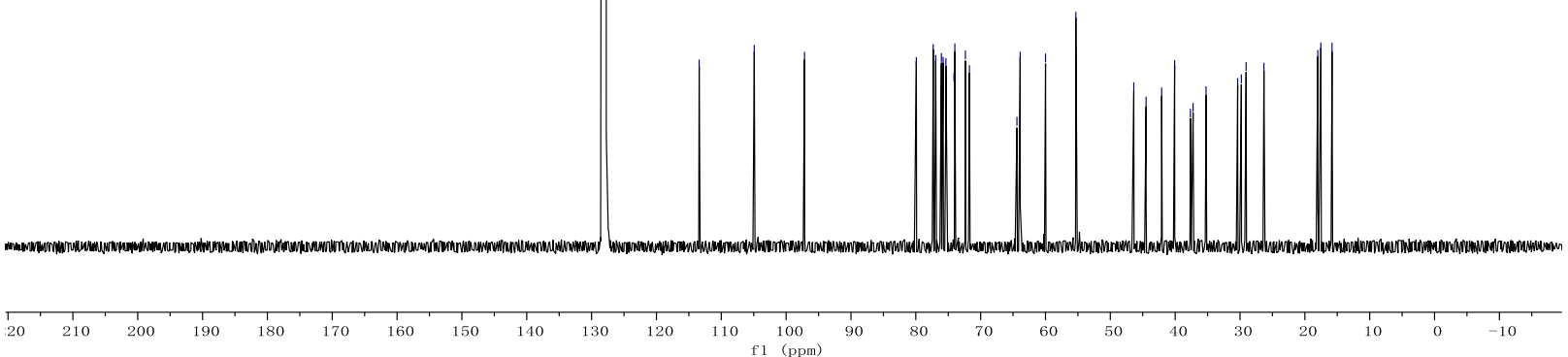
${ }^{1} \mathrm{H}$ NMR (600 MHz, $\mathrm{C}_{6} \mathrm{D}_{6}$ ) of 47 (zoom in 5.3-2.6 ppm)
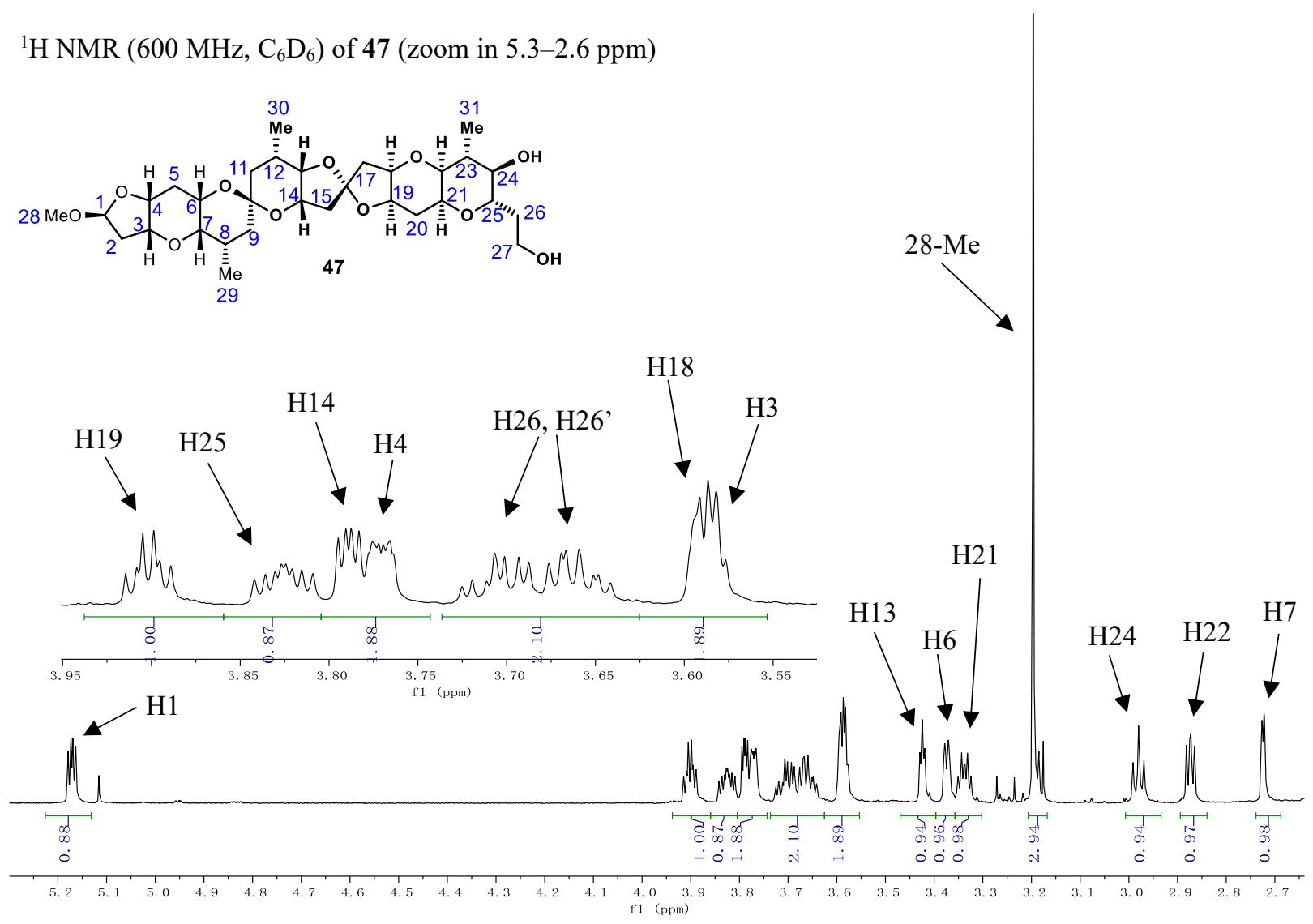

${ }^{1} \mathrm{H}$ NMR $\left(600 \mathrm{MHz}, \mathrm{C}_{6} \mathrm{D}_{6}\right)$ of 47 (zoom in $2.7-0.8 \mathrm{ppm}$ )
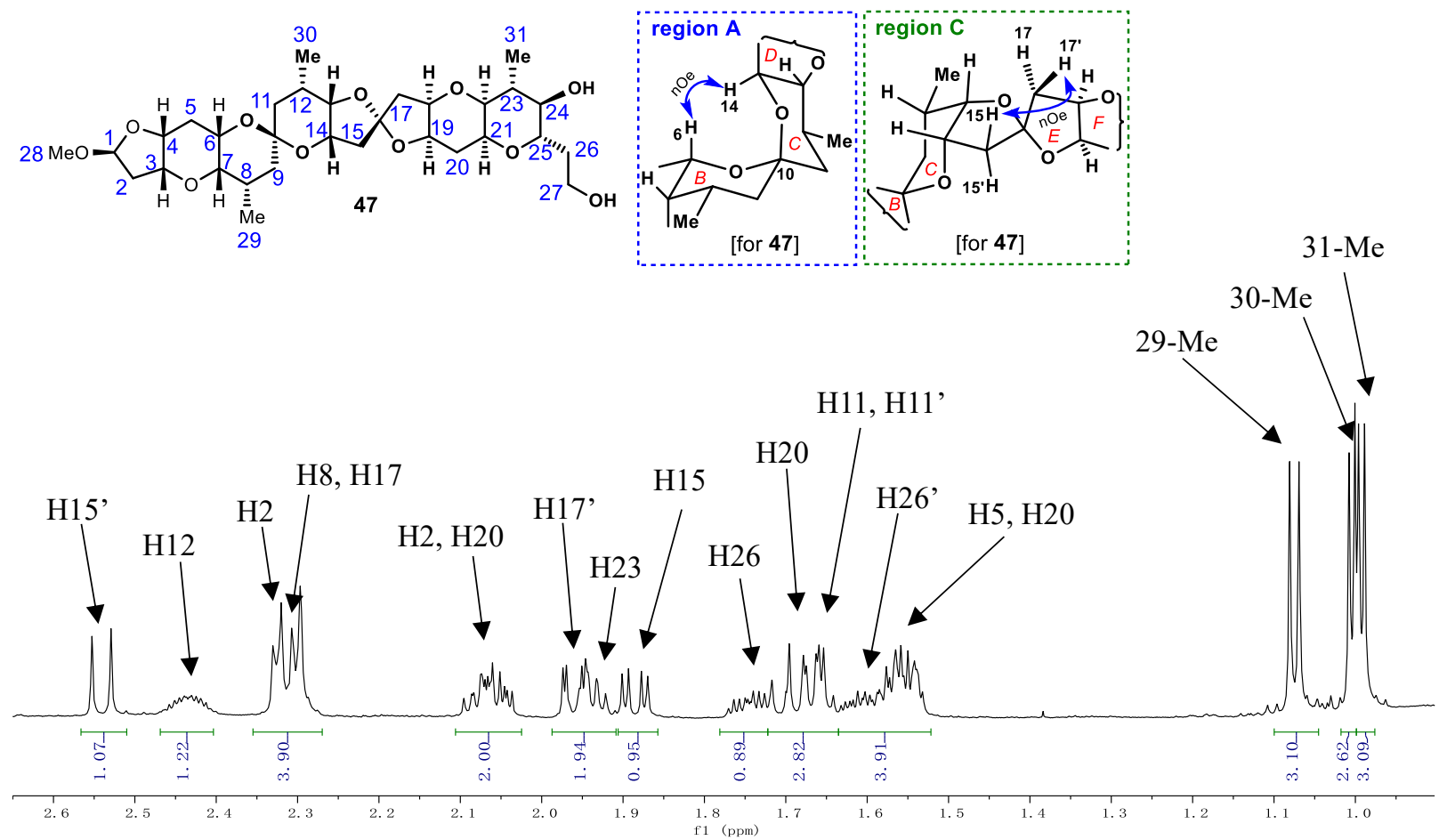


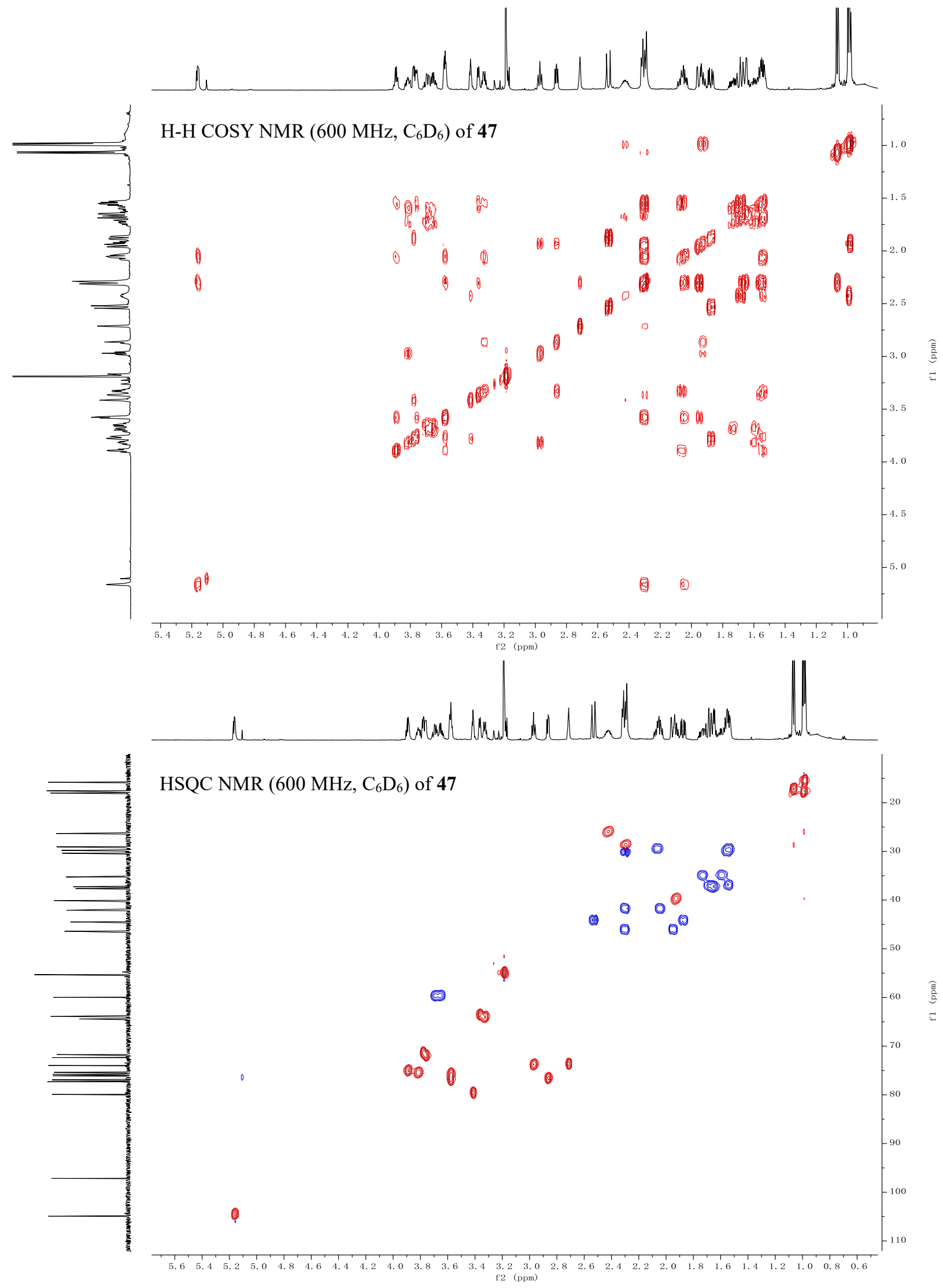




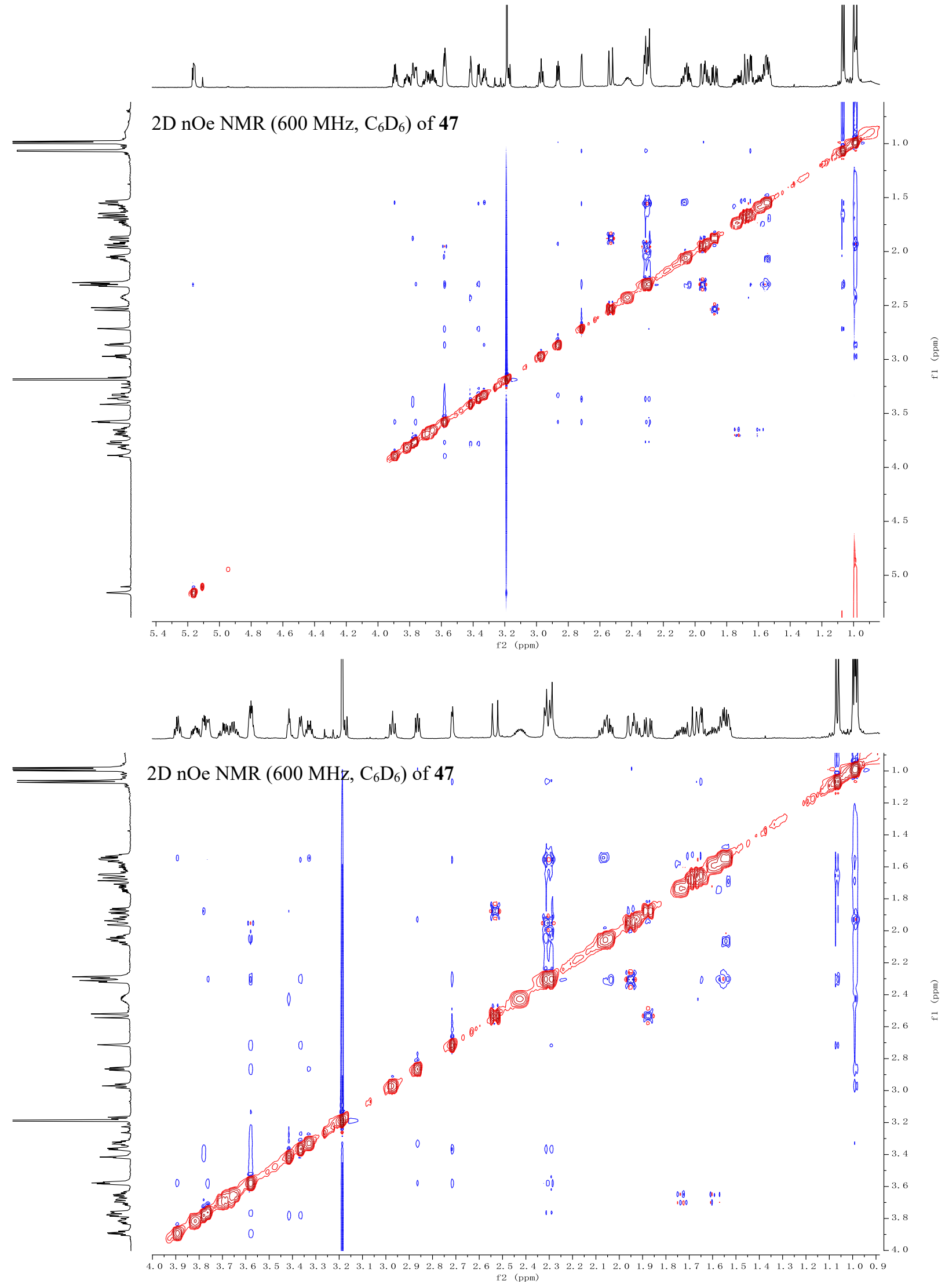


${ }^{1} \mathrm{H}$ NMR (600 MHz, $\left.\mathrm{C}_{6} \mathrm{D}_{6}\right)$ of C16-epi-47
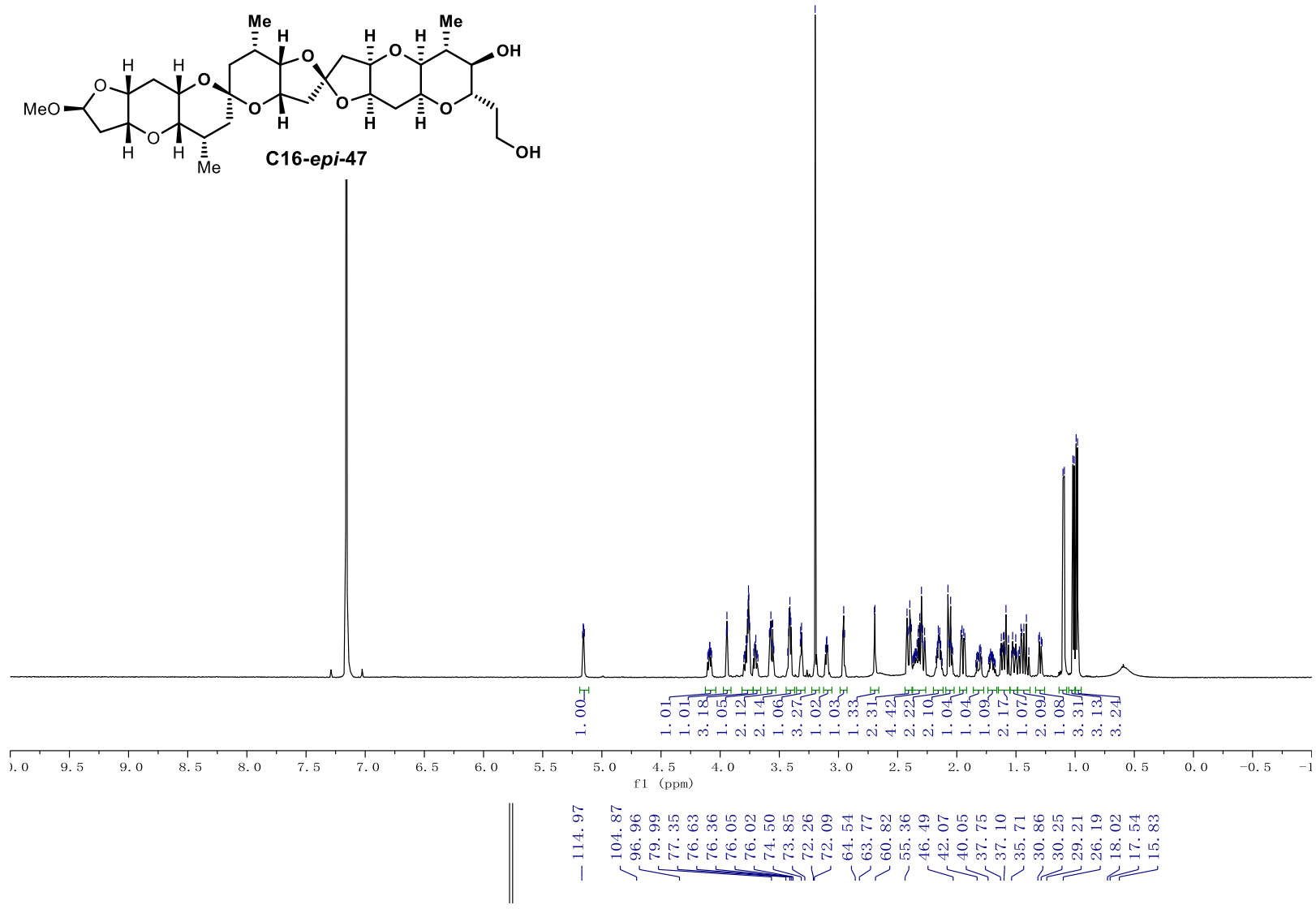

${ }^{13} \mathrm{C}$ NMR (151 MHz, $\mathrm{C}_{6} \mathrm{D}_{6}$ ) of C16-epi-47
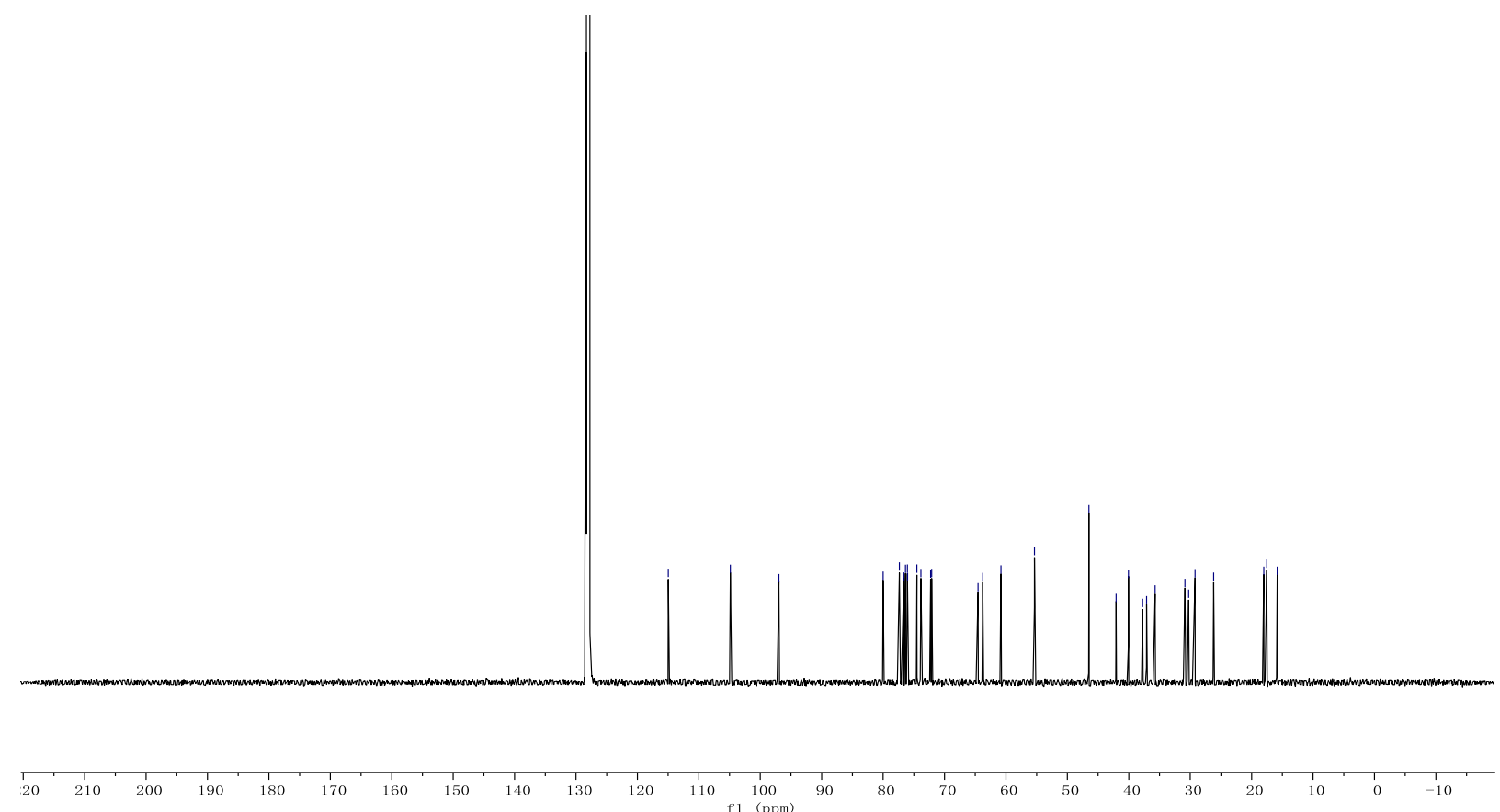
${ }^{1} \mathrm{H}$ NMR (600 MHz, $\mathrm{C}_{6} \mathrm{D}_{6}$ ) of C16-epi-47 (zoom in 5.5-2.5 ppm)
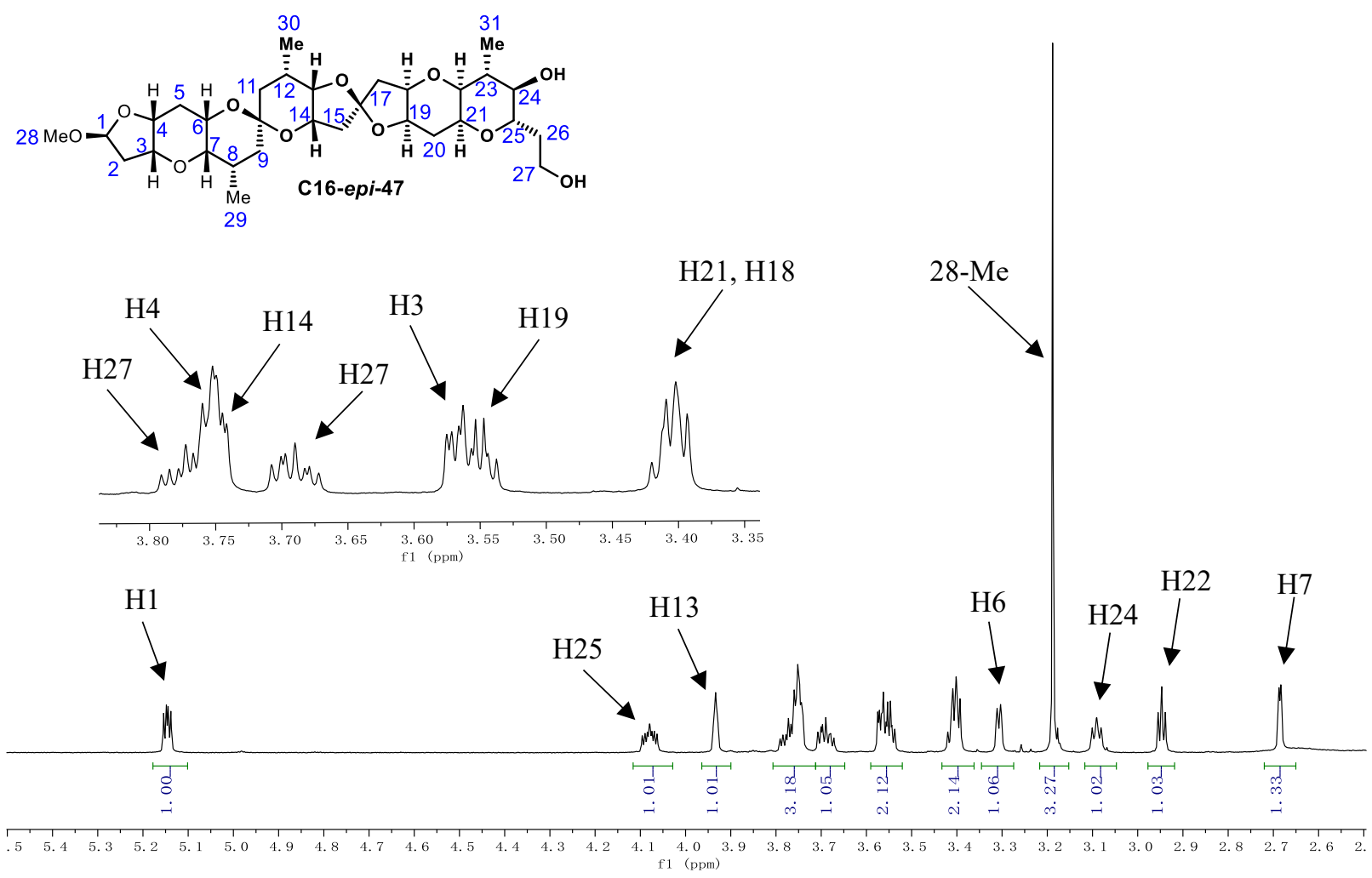

${ }^{1} \mathrm{H}$ NMR (600 MHz, $\mathrm{C}_{6} \mathrm{D}_{6}$ ) of C16-epi-47 (zoom in 2.6-0.8 ppm)
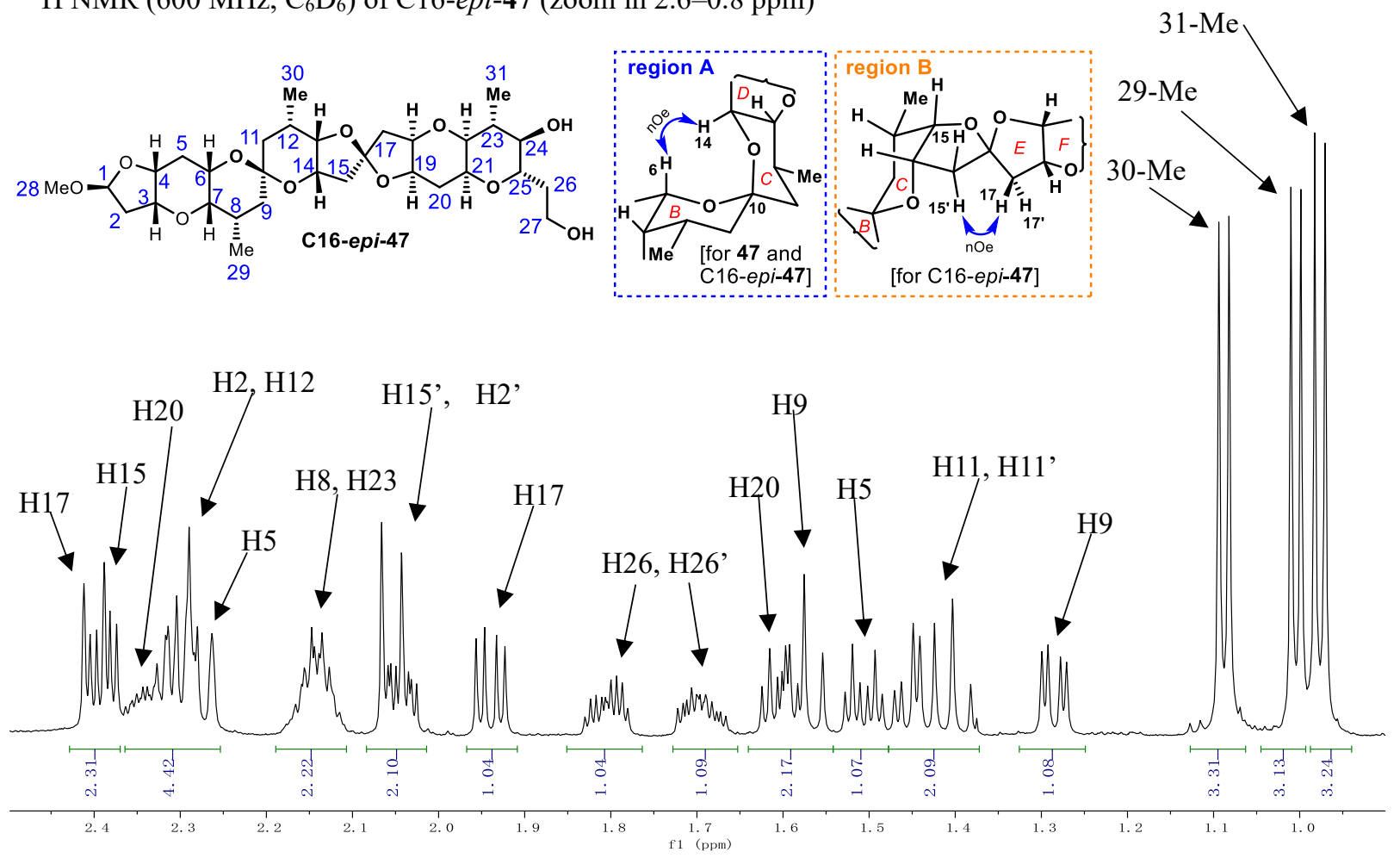


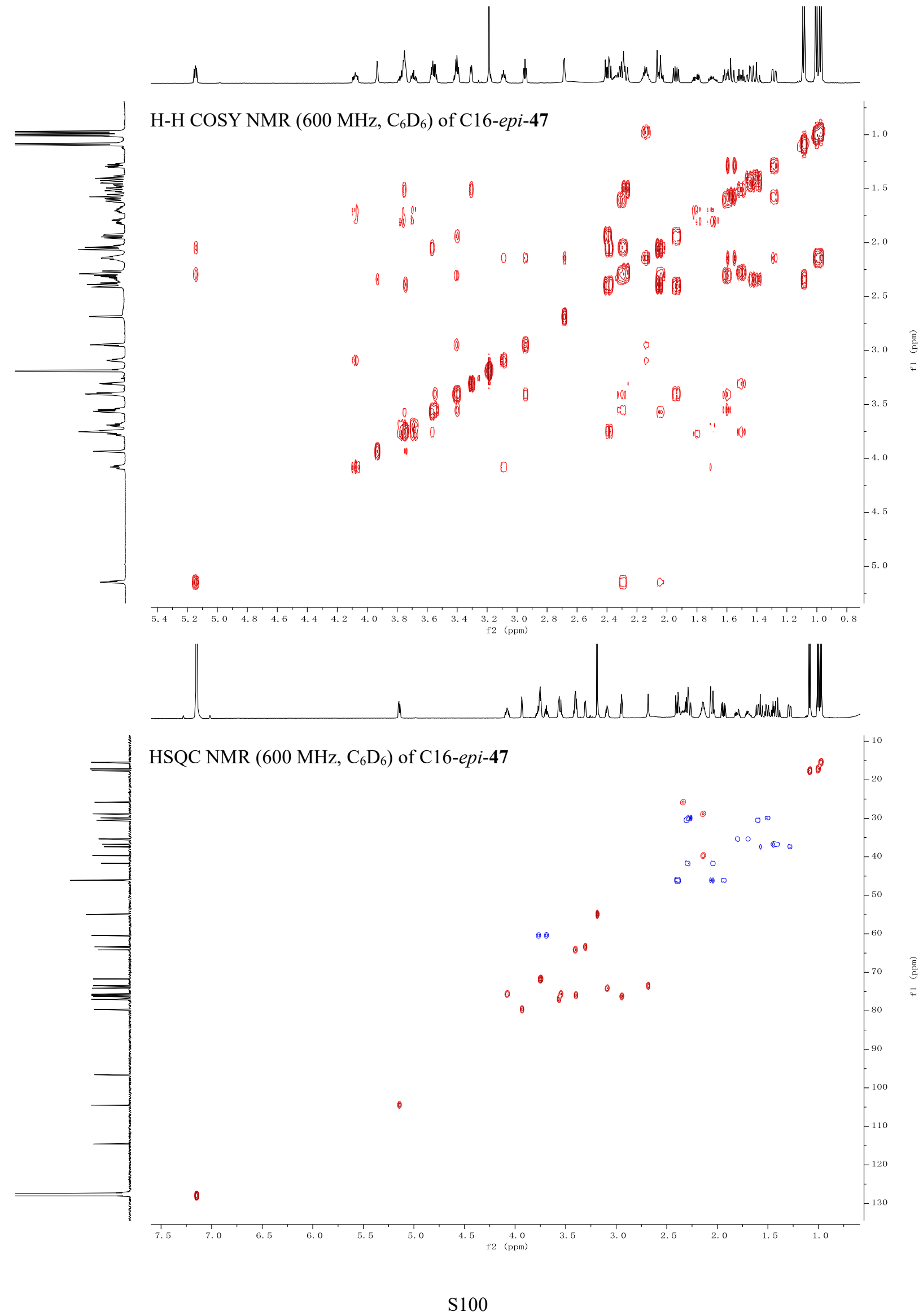



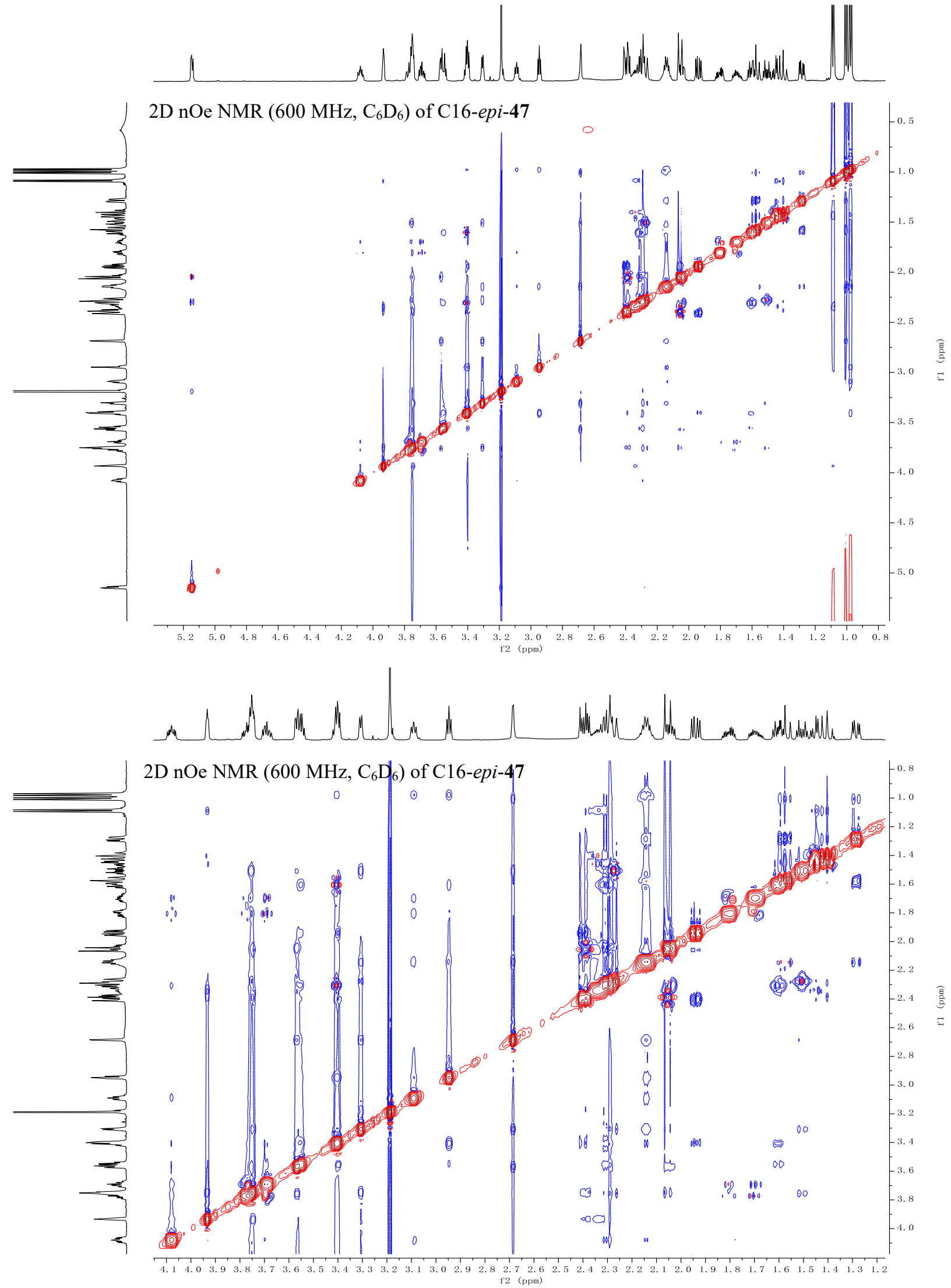
${ }^{1} \mathrm{H}$ NMR $\left(600 \mathrm{MHz}, \mathrm{C}_{6} \mathrm{D}_{6}\right)$ of C16-epi-30
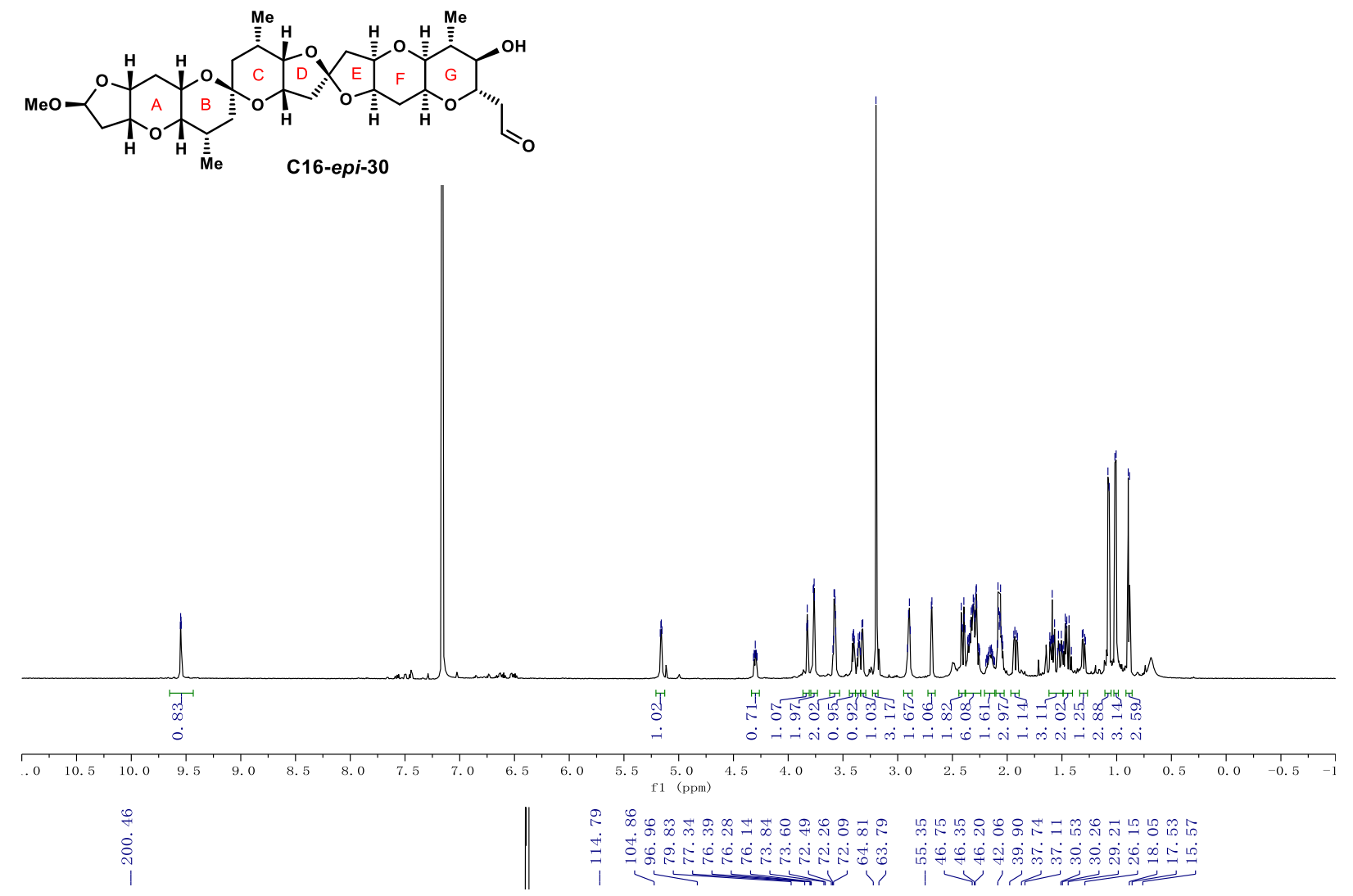

${ }^{13} \mathrm{C}$ NMR (151 MHz, $\left.\mathrm{C}_{6} \mathrm{D}_{6}\right)$ of C16-epi-30

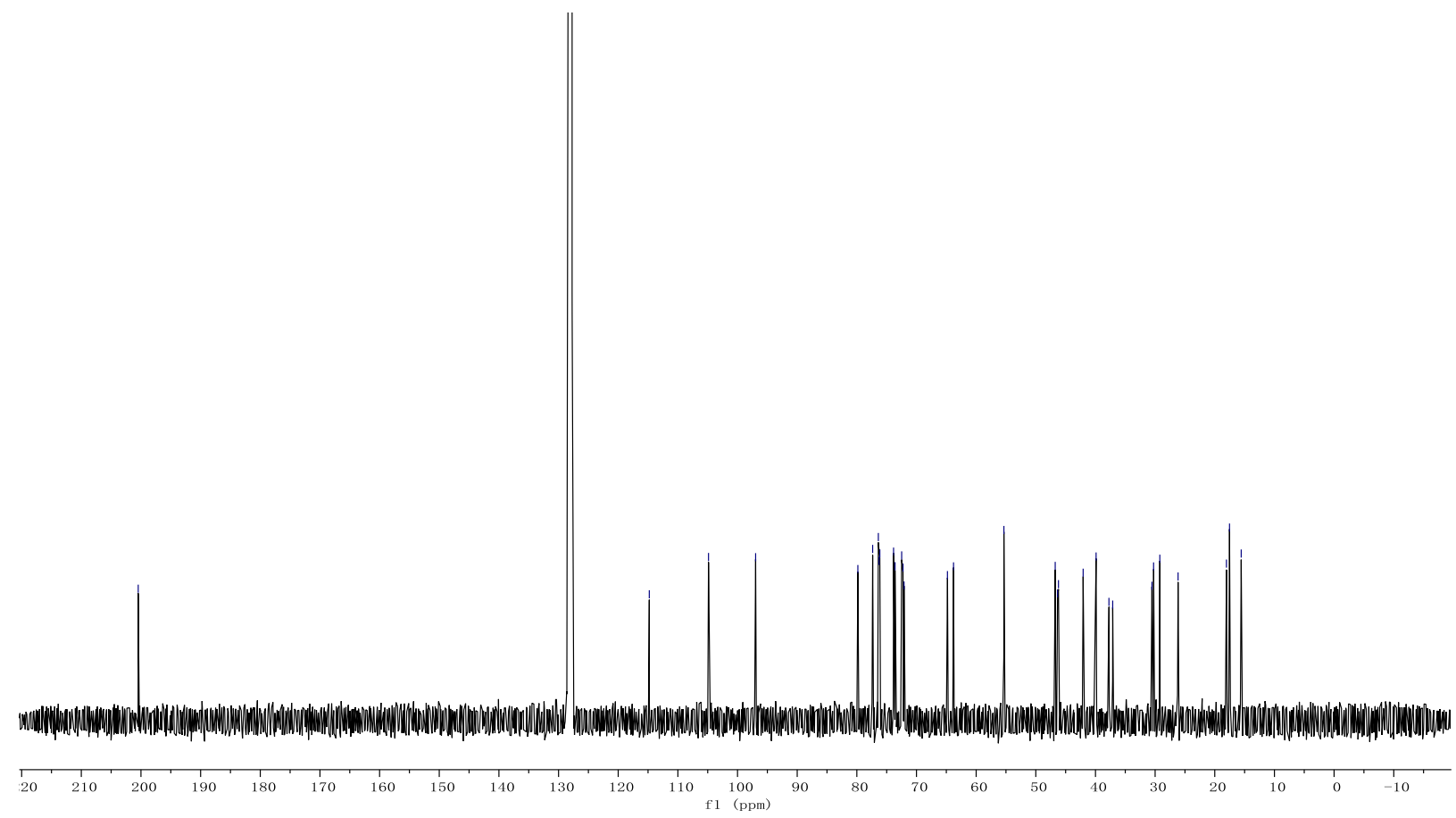


${ }^{1} \mathrm{H}$ NMR $\left(600 \mathrm{MHz}, \mathrm{CDCl}_{3}\right)$ of $\mathbf{S 6}$
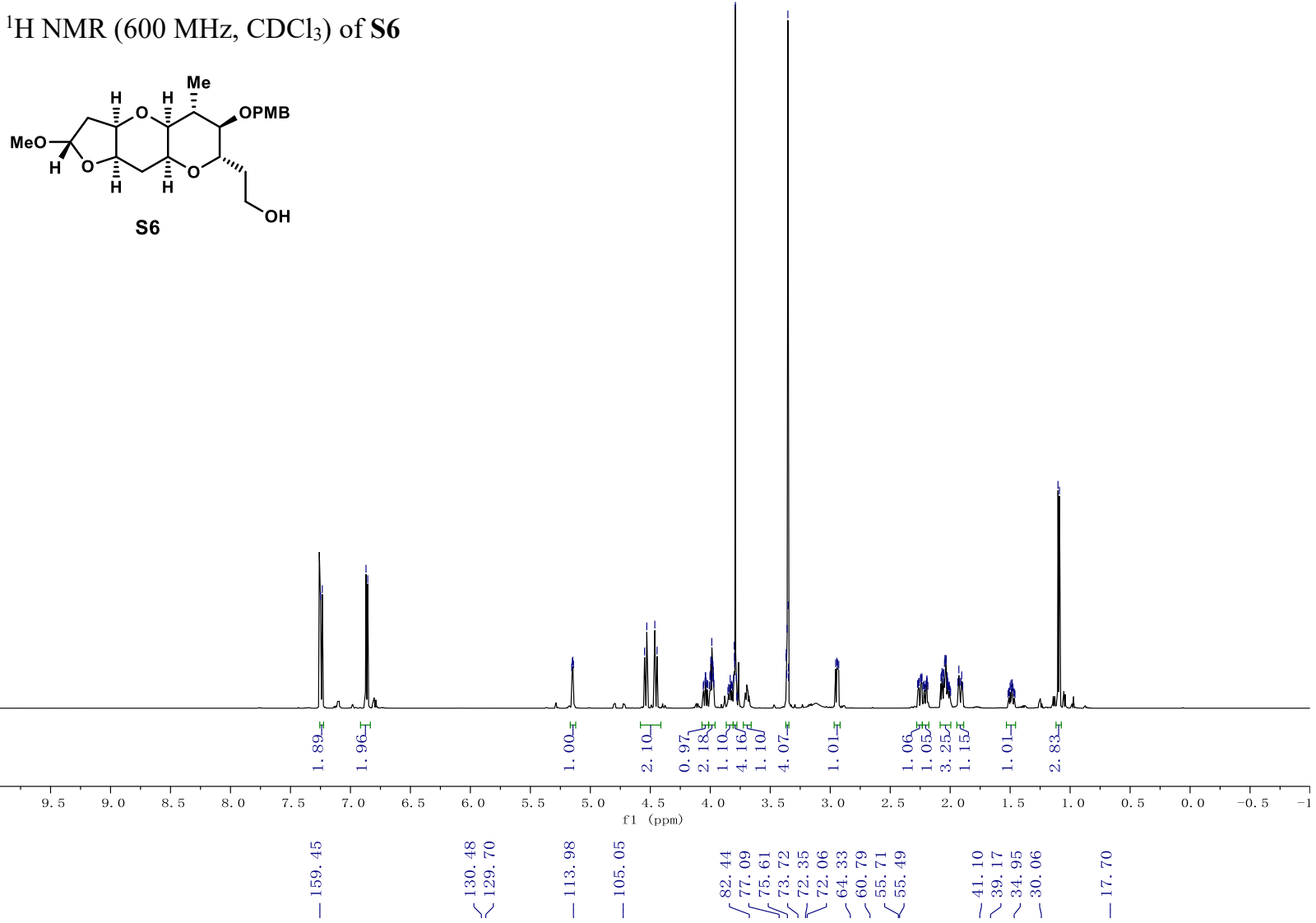

${ }^{13} \mathrm{C}$ NMR $\left(151 \mathrm{MHz}, \mathrm{CDCl}_{3}\right)$ of $\mathbf{S 6}$
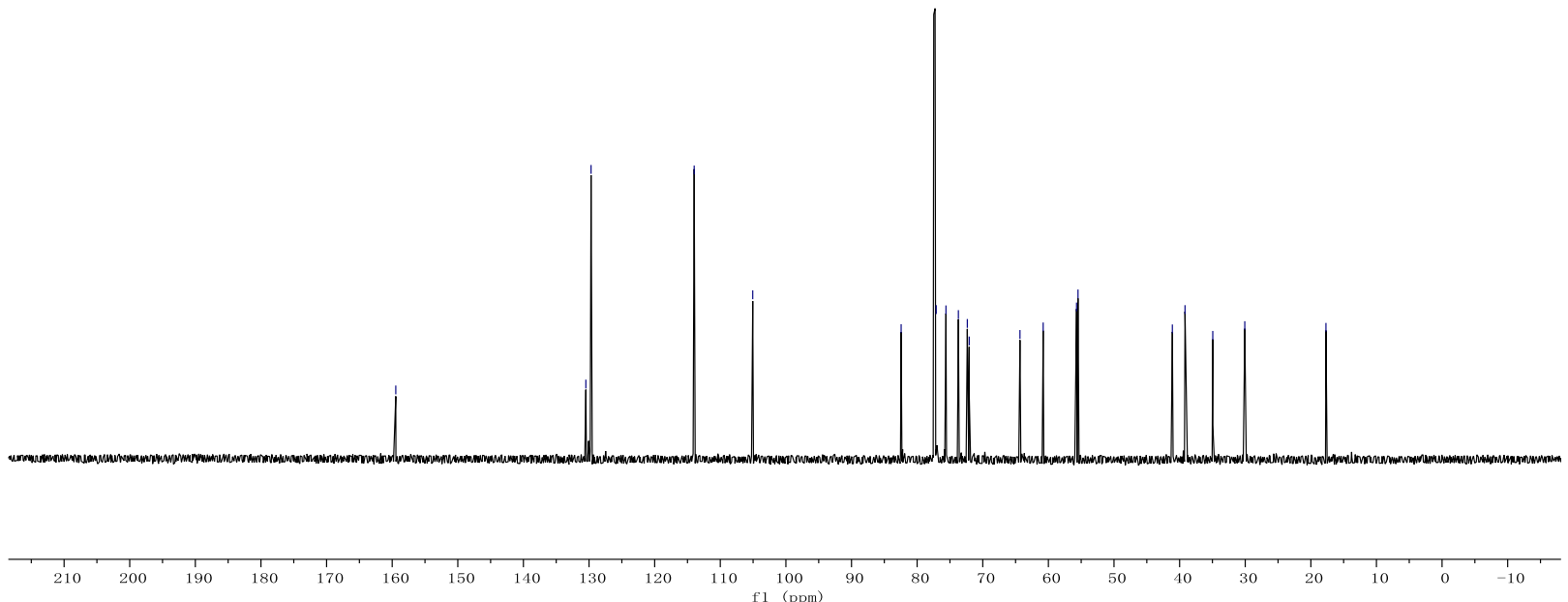
${ }^{1} \mathrm{H}$ NMR $\left(600 \mathrm{MHz}, \mathrm{CDCl}_{3}\right)$ of $\mathbf{4 8 b}$

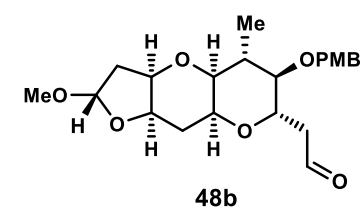

48b

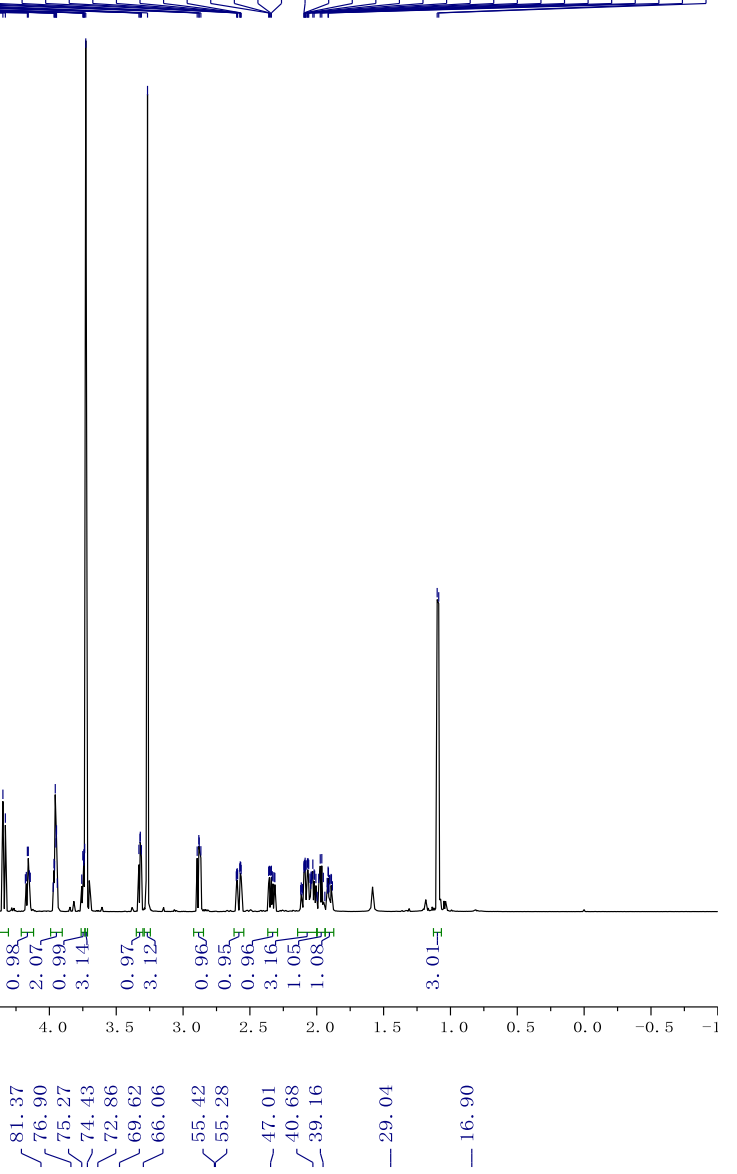

${ }^{13} \mathrm{C}$ NMR $\left(151 \mathrm{MHz}, \mathrm{CDCl}_{3}\right)$ of $\mathbf{4 8 b}$
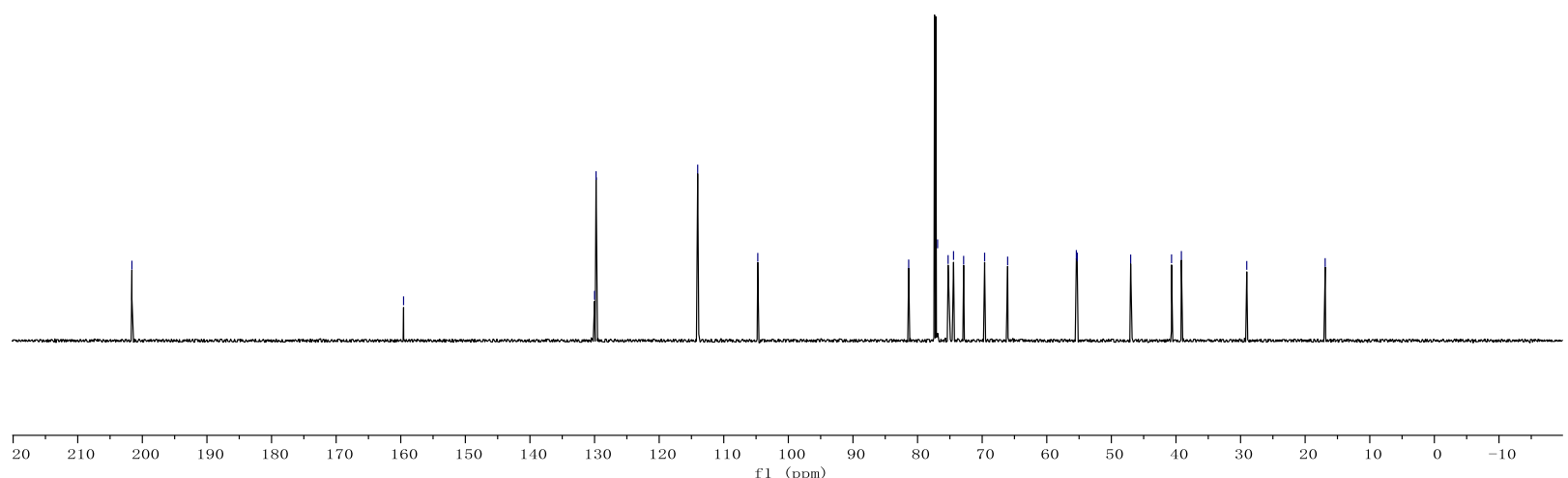
${ }^{1} \mathrm{H}$ NMR $\left(600 \mathrm{MHz}, \mathrm{CDCl}_{3}\right)$ of $\mathbf{S 7}$
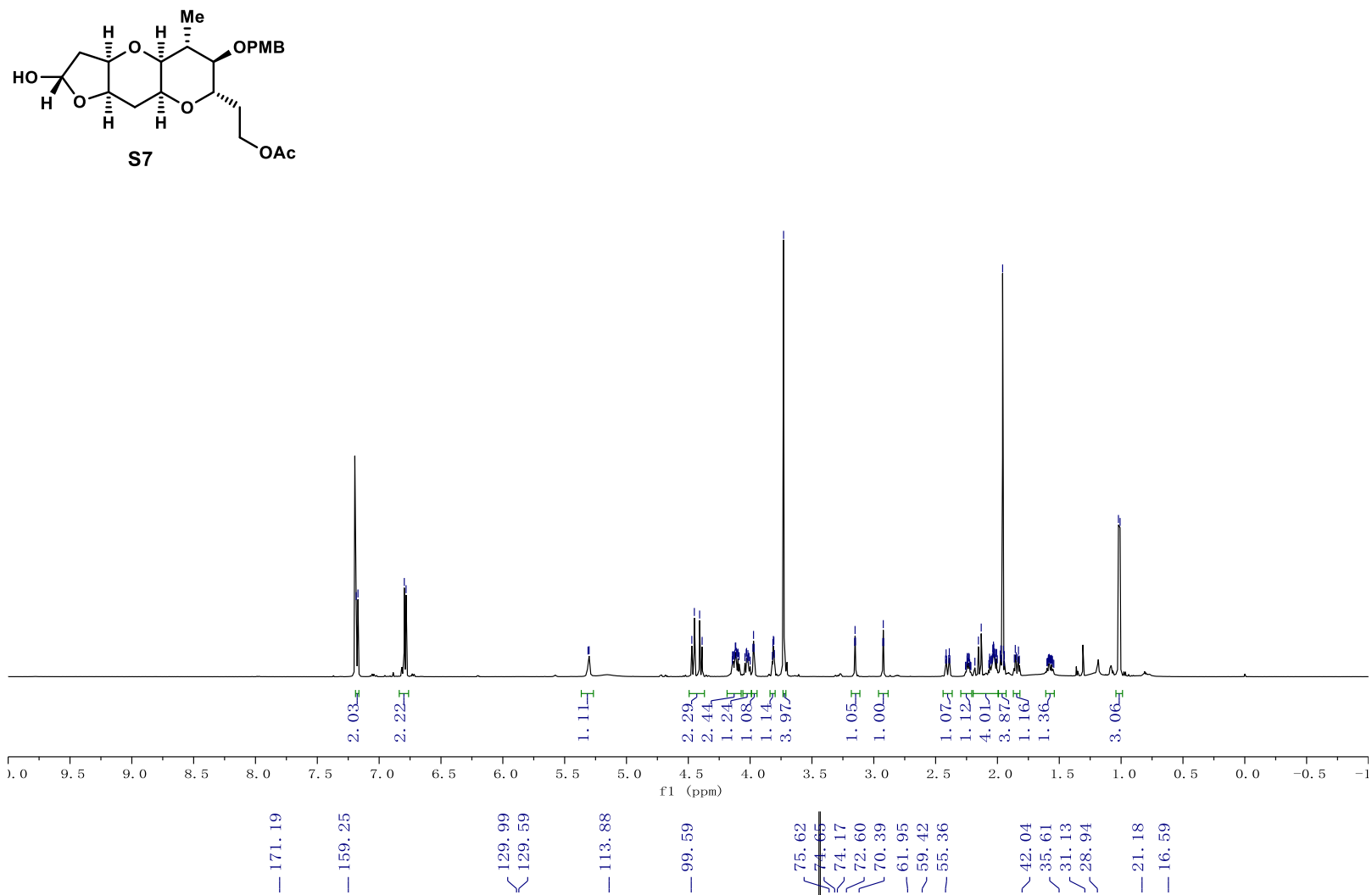

${ }^{13} \mathrm{C}$ NMR $\left(151 \mathrm{MHz}, \mathrm{CDCl}_{3}\right)$ of $\mathbf{S} 7$
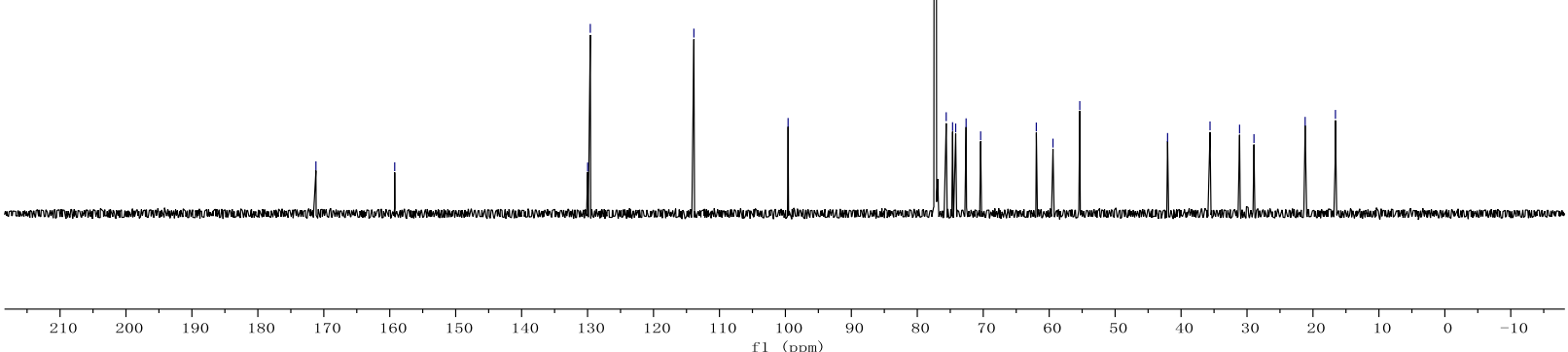
${ }^{1} \mathrm{H}$ NMR $\left(600 \mathrm{MHz}, \mathrm{CDCl}_{3}\right)$ of $\mathbf{S 8}$
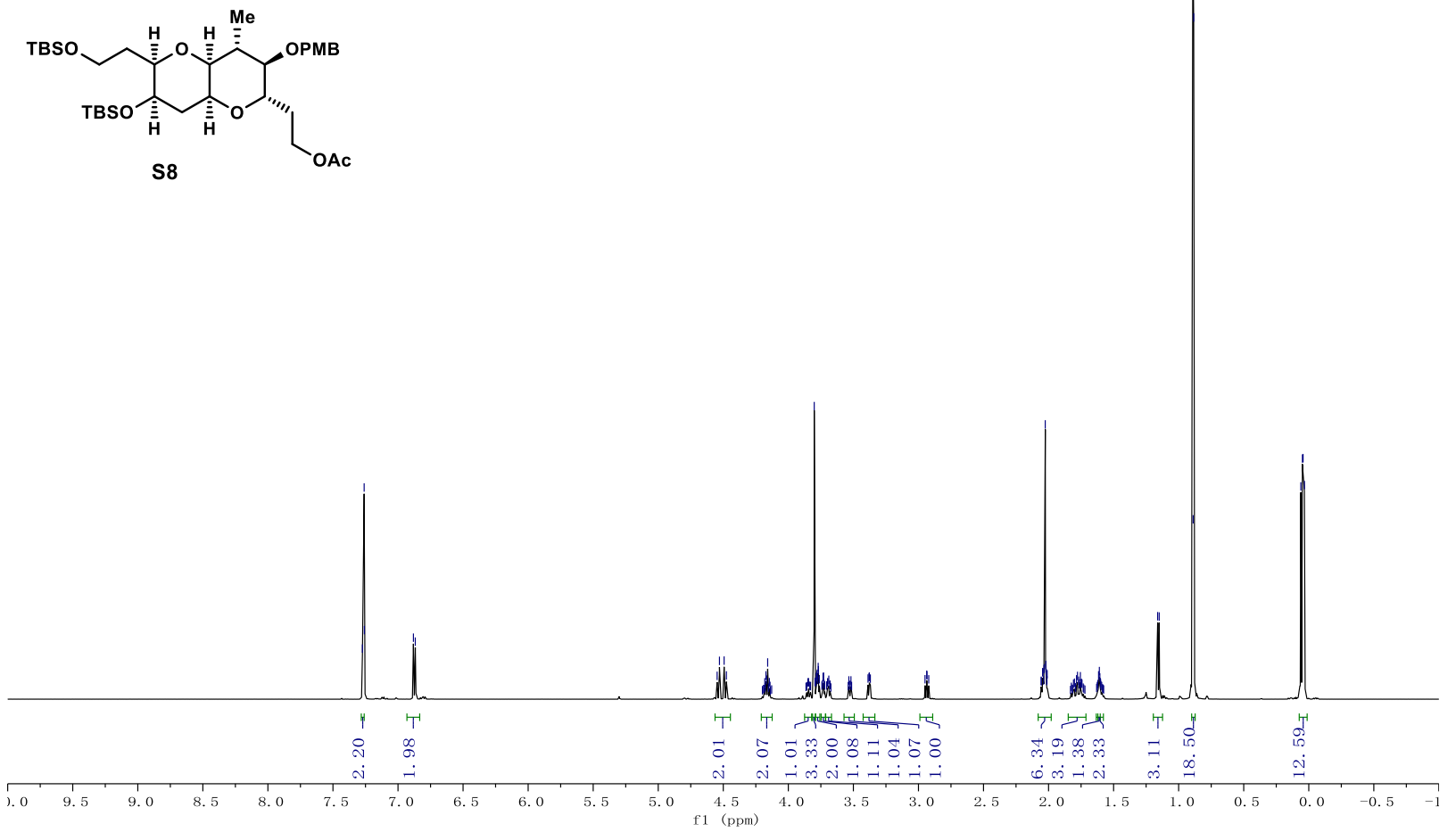

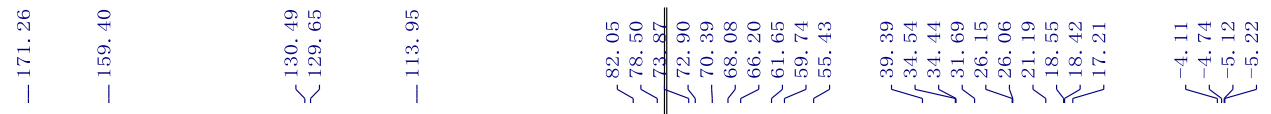

${ }^{13} \mathrm{C}$ NMR $\left(151 \mathrm{MHz}, \mathrm{CDCl}_{3}\right)$ of $\mathbf{S 8}$

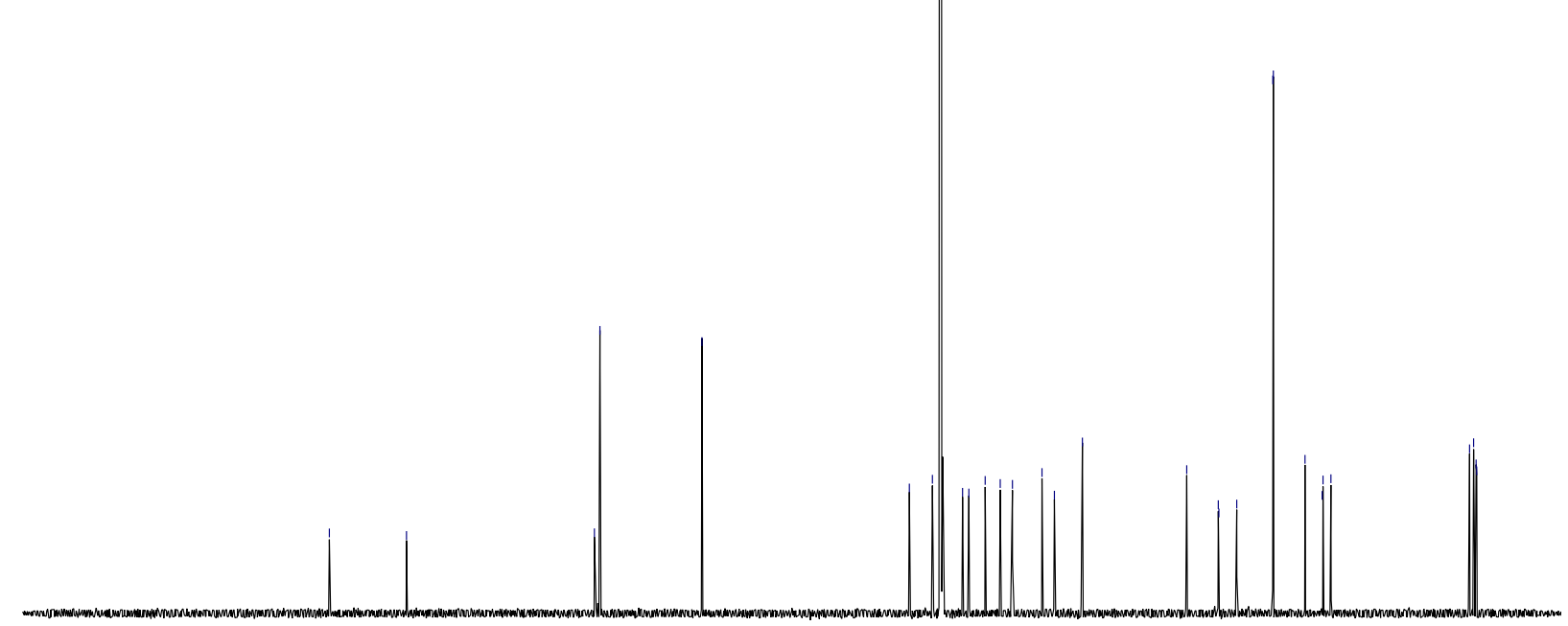


${ }^{1} \mathrm{H}$ NMR $\left(600 \mathrm{MHz}, \mathrm{CDCl}_{3}\right)$ of $\mathbf{4 8 a}$
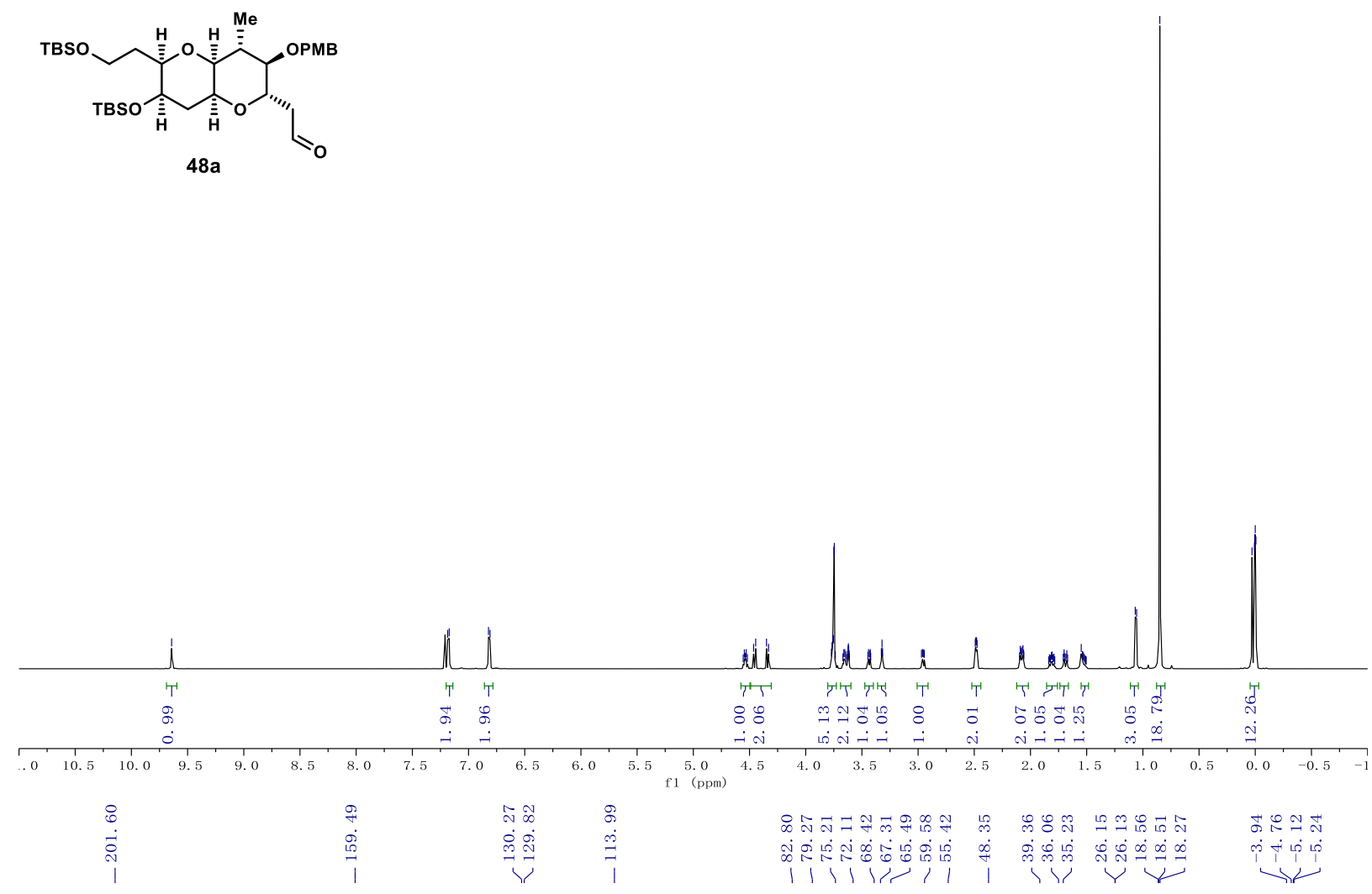

${ }^{13} \mathrm{C} \mathrm{NMR}\left(151 \mathrm{MHz}, \mathrm{CDCl}_{3}\right)$ of 48a
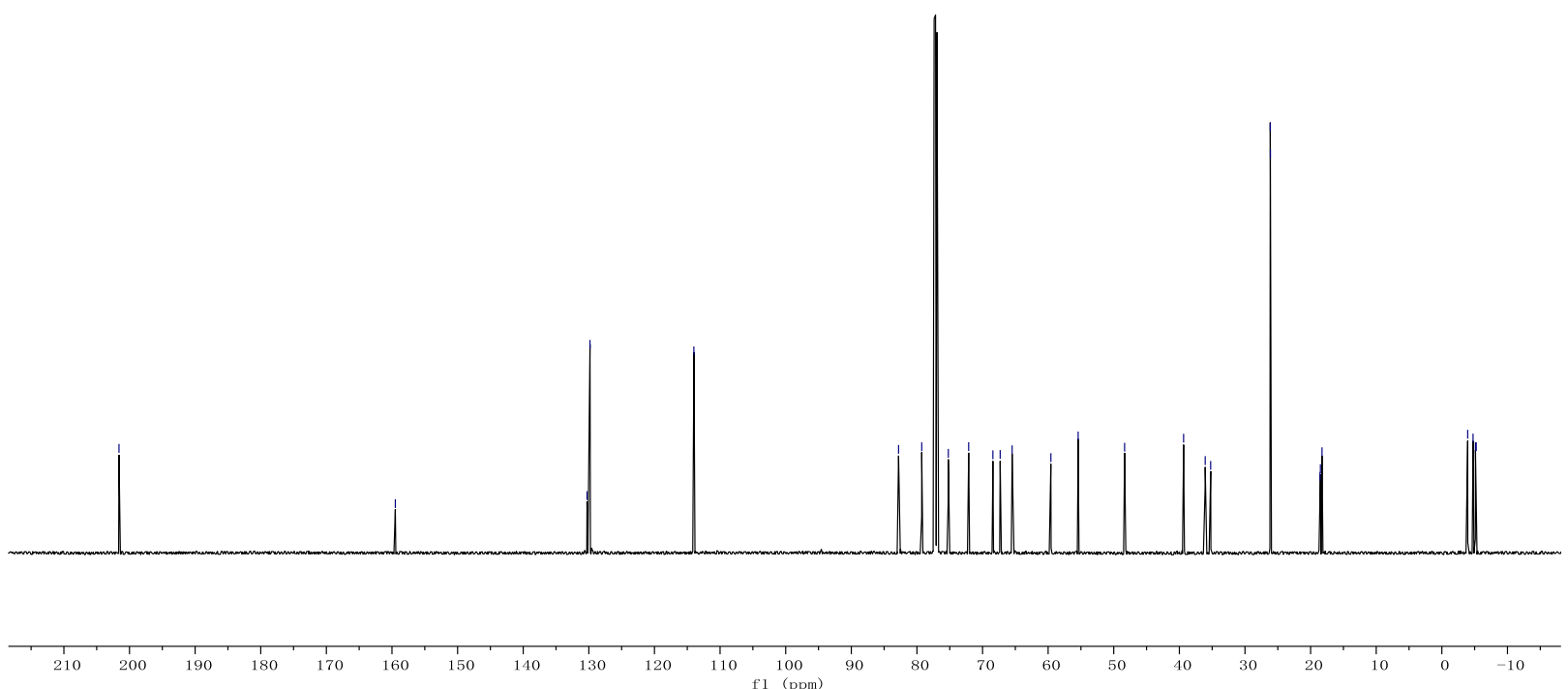
${ }^{1} \mathrm{H}$ NMR $\left(600 \mathrm{MHz}, \mathrm{C}_{6} \mathrm{D}_{6}\right)$ of de-I-31

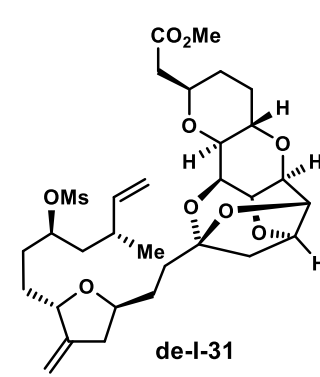

$$
\text { (1) }
$$

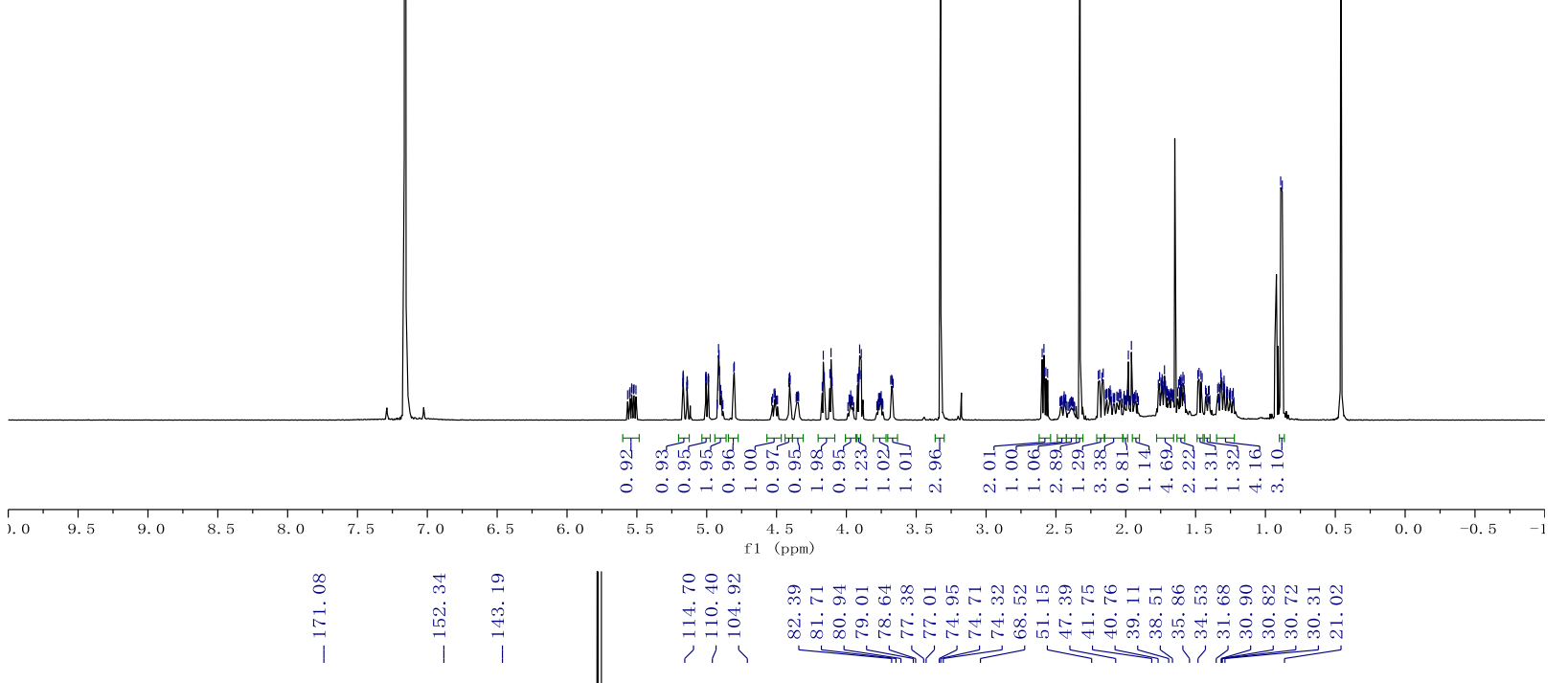

${ }^{13} \mathrm{C}$ NMR (151 MHz, $\left.\mathrm{C}_{6} \mathrm{D}_{6}\right)$ of de-I-31
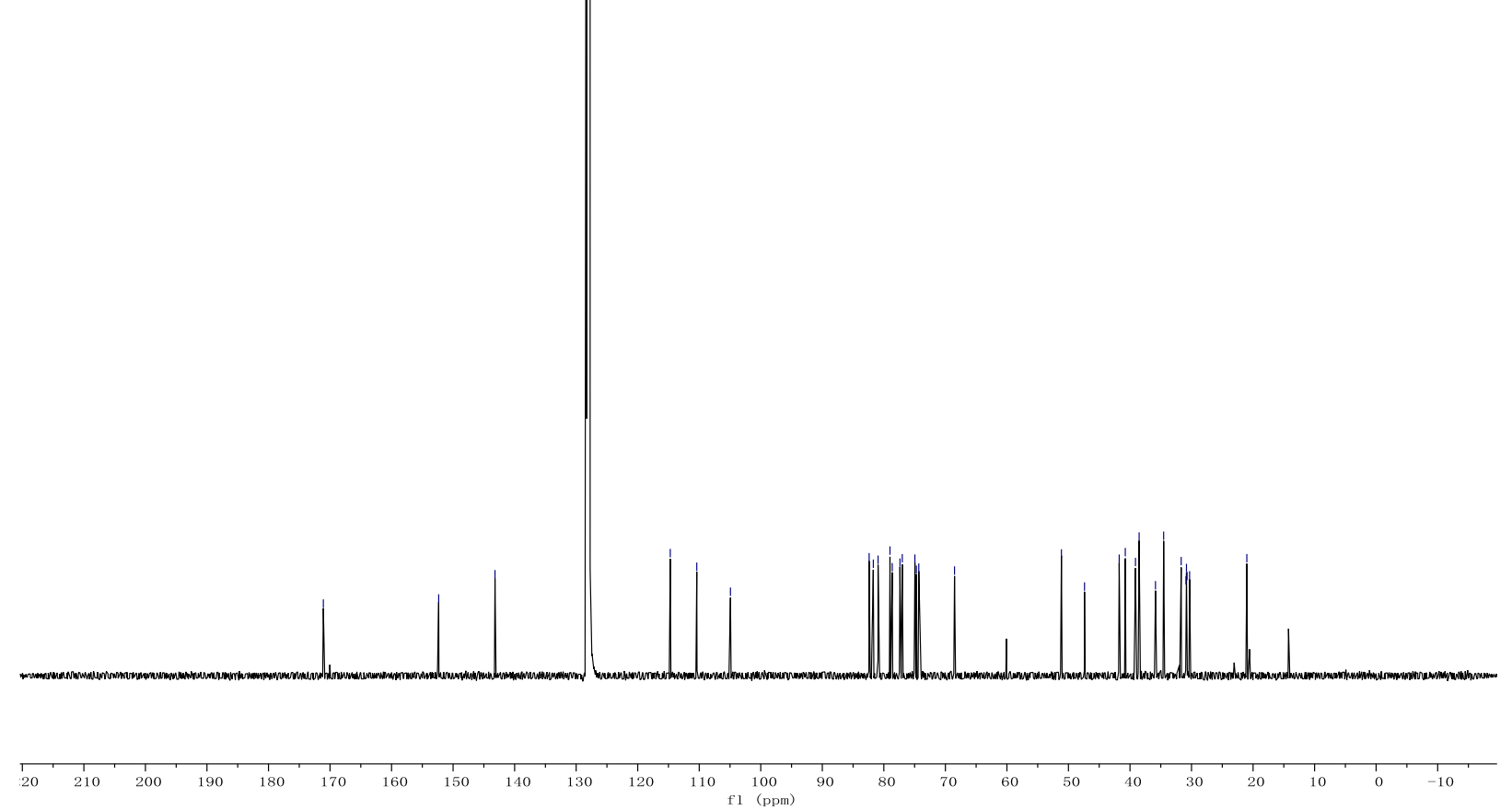
${ }^{1} \mathrm{H} \mathrm{NMR}\left(600 \mathrm{MHz}, \mathrm{CDCl}_{3}\right)$ of $\mathbf{5 0 a}$

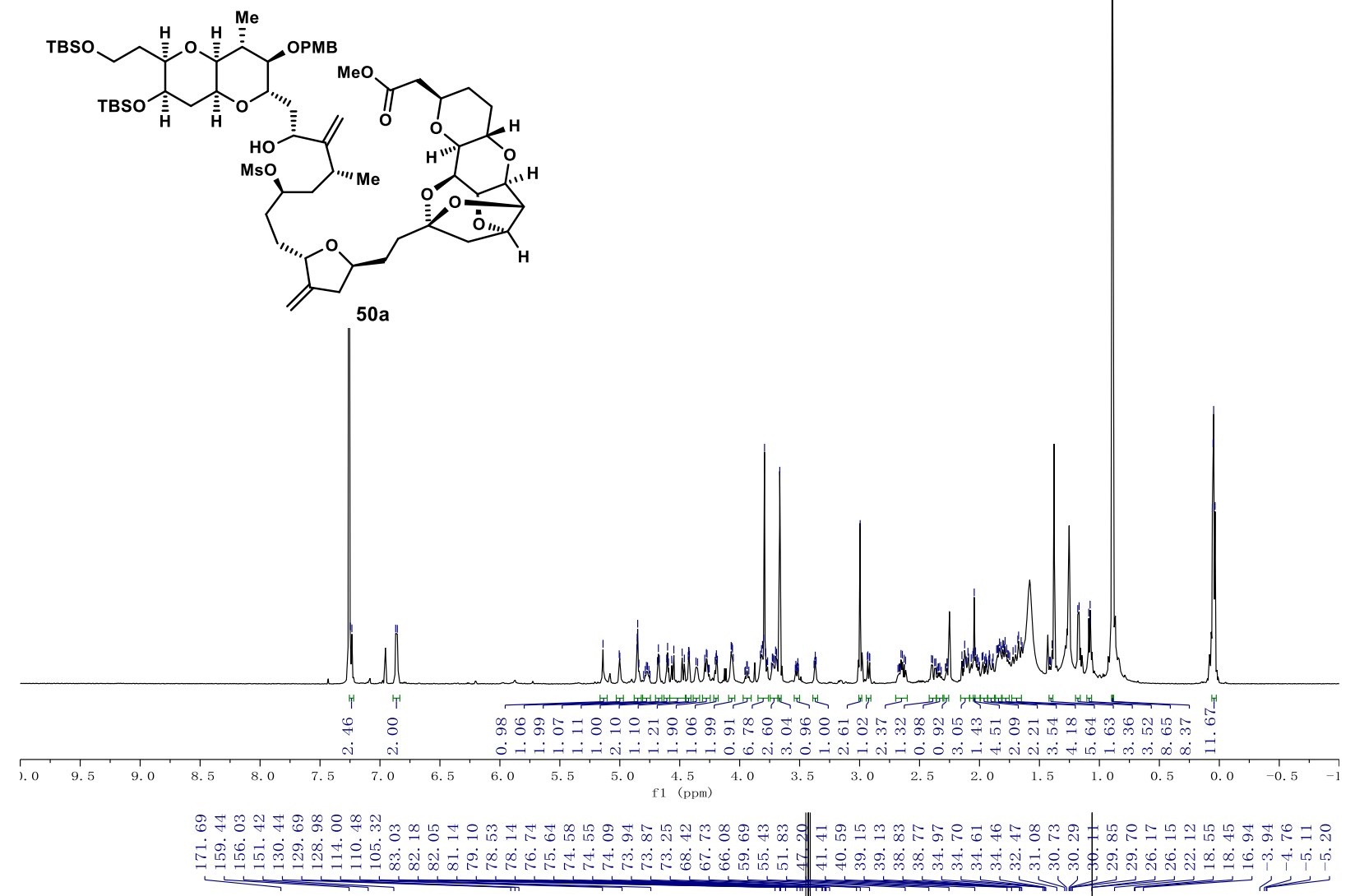

${ }^{13} \mathrm{C}$ NMR $\left(151 \mathrm{MHz}, \mathrm{CDCl}_{3}\right)$ of $\mathbf{5 0 a}$
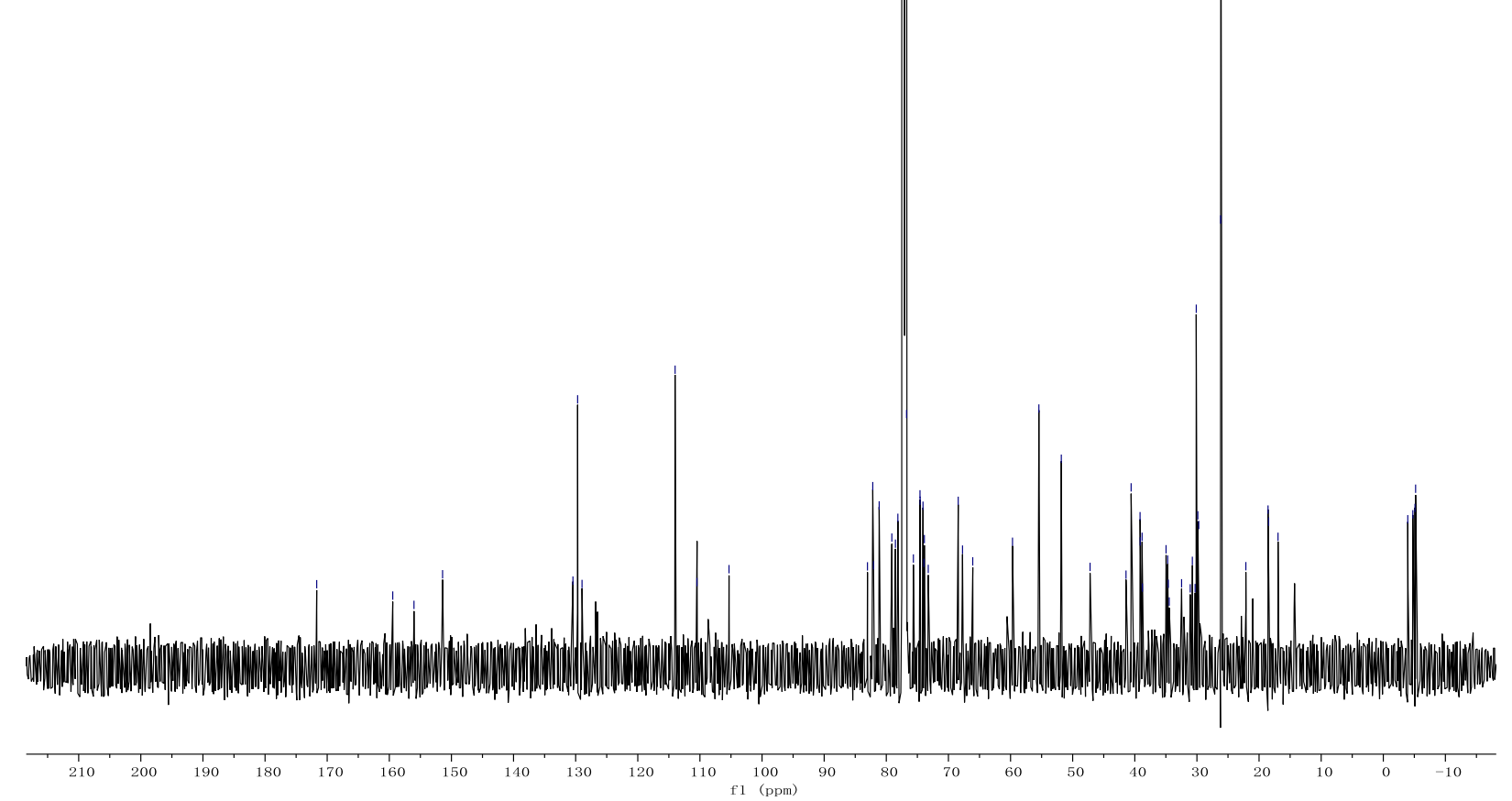
${ }^{1} \mathrm{H}$ NMR $\left(600 \mathrm{MHz}, \mathrm{CDCl}_{3}\right)$ of $\mathbf{S 9}$

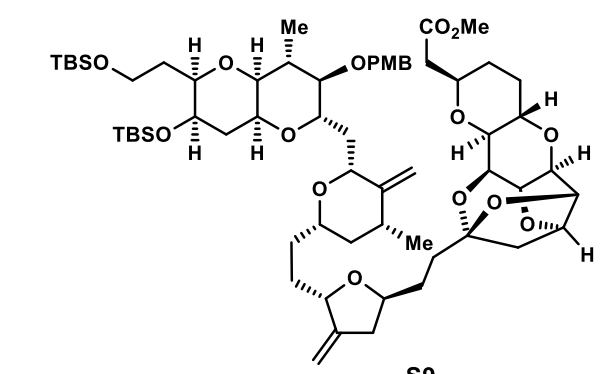

s9

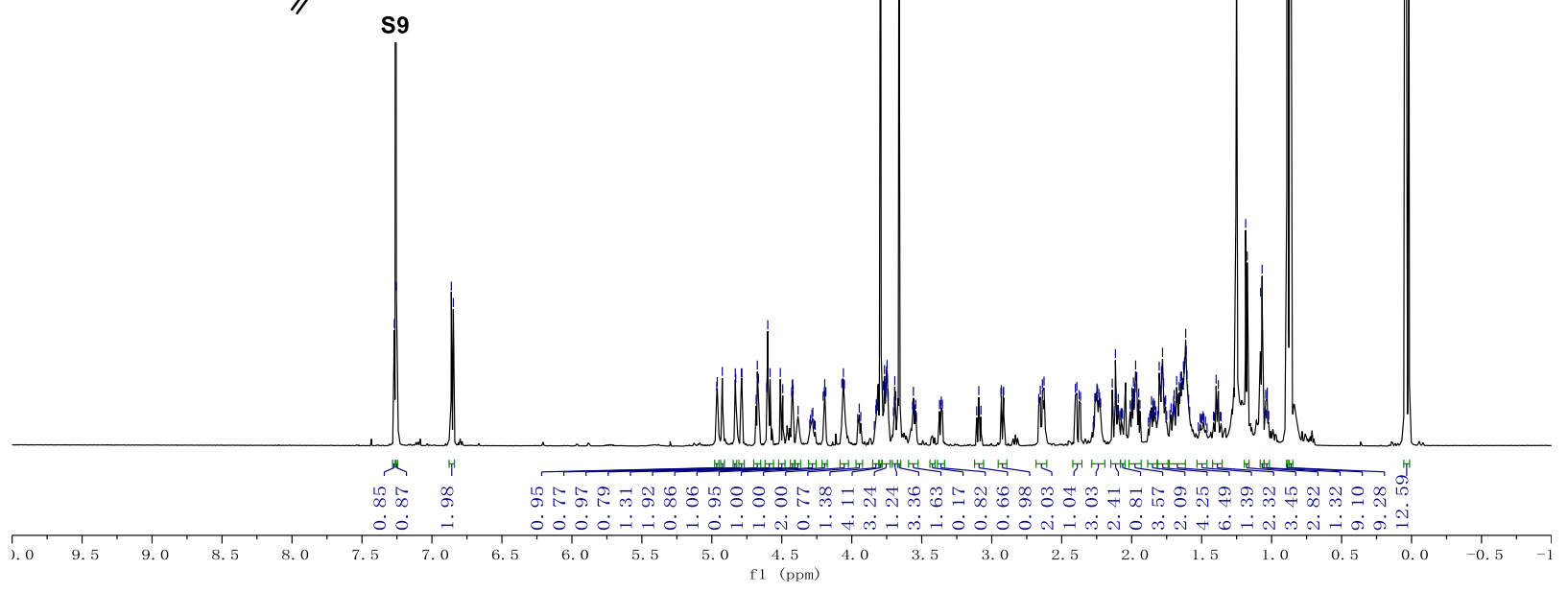

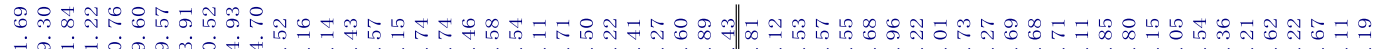

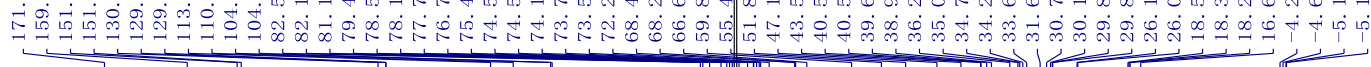

${ }^{13} \mathrm{C}$ NMR $\left(151 \mathrm{MHz}, \mathrm{CDCl}_{3}\right)$ of $\mathbf{S 9}$

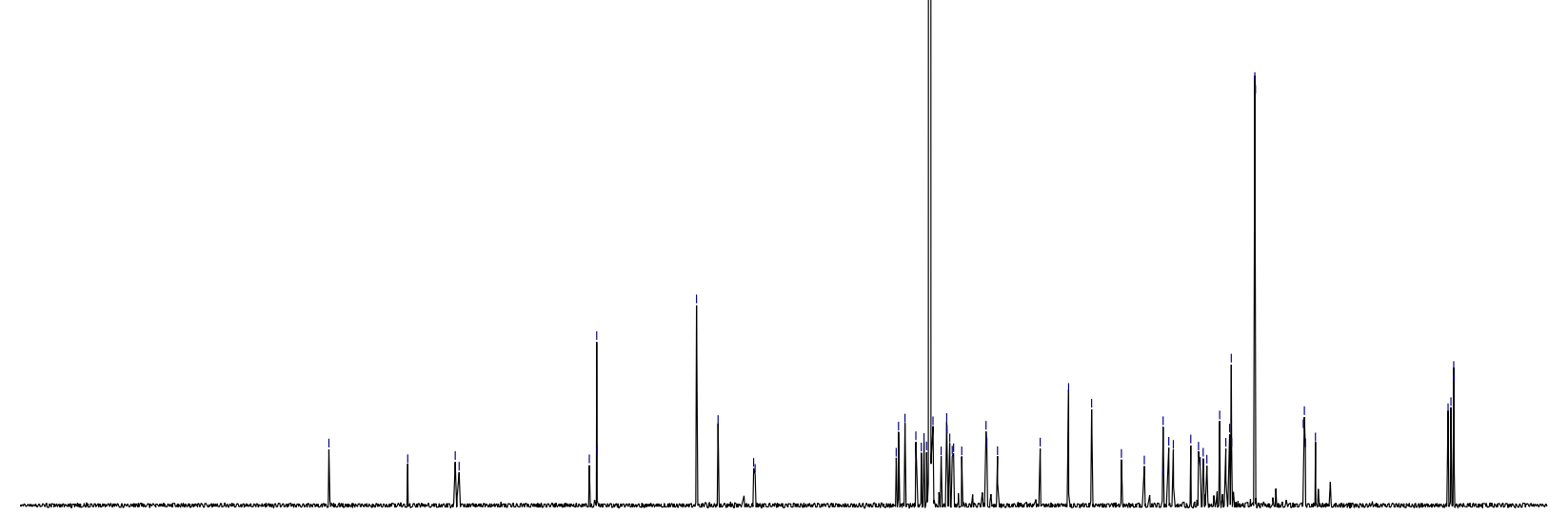

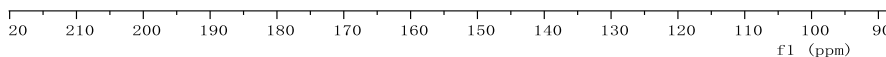


${ }^{1} \mathrm{H}$ NMR $\left(600 \mathrm{MHz}, \mathrm{C}_{6} \mathrm{D}_{6}\right)$ of C12-epi-50b

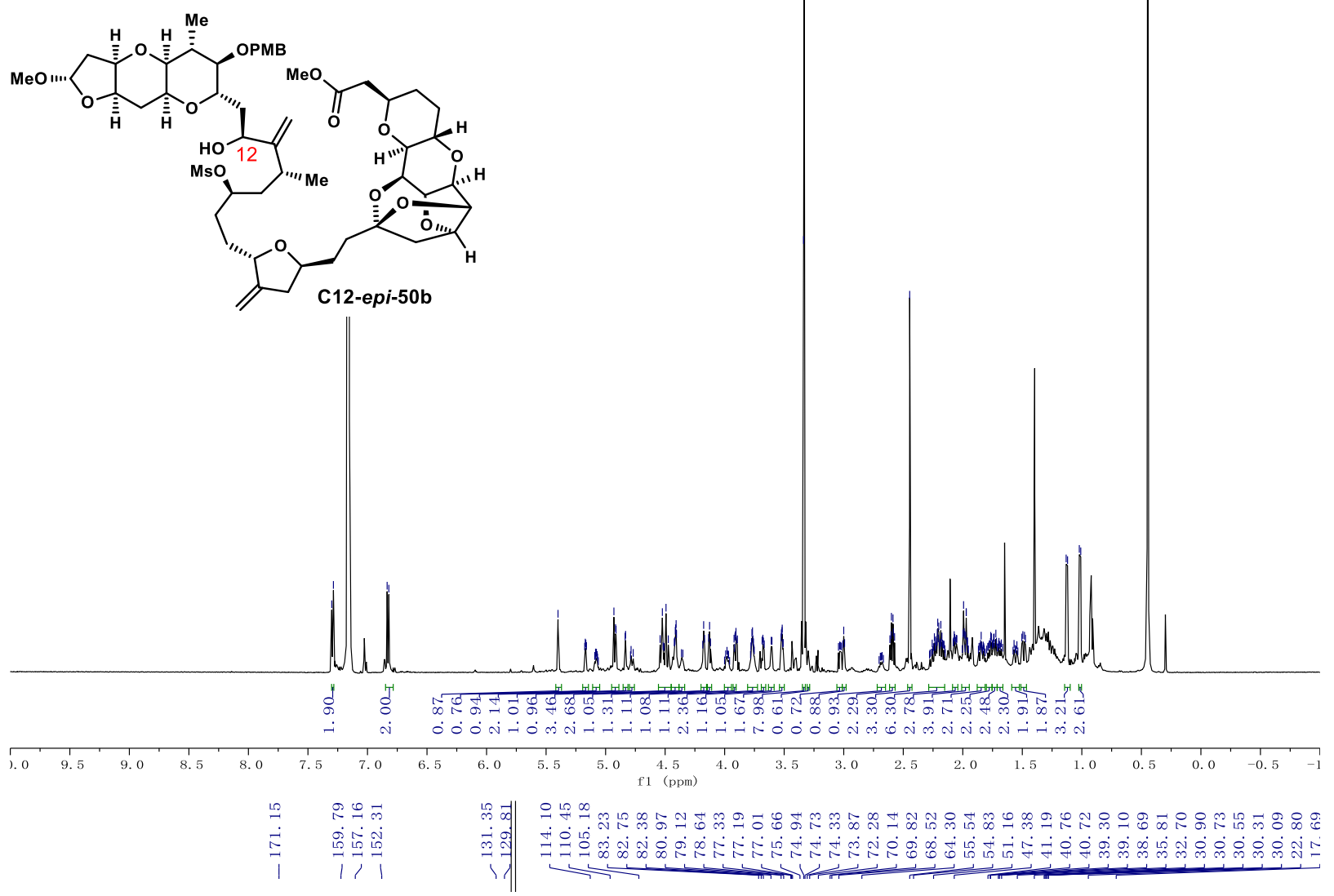

${ }^{13} \mathrm{C}$ NMR (151 MHz, $\left.\mathrm{C}_{6} \mathrm{D}_{6}\right)$ of C12-epi-50b
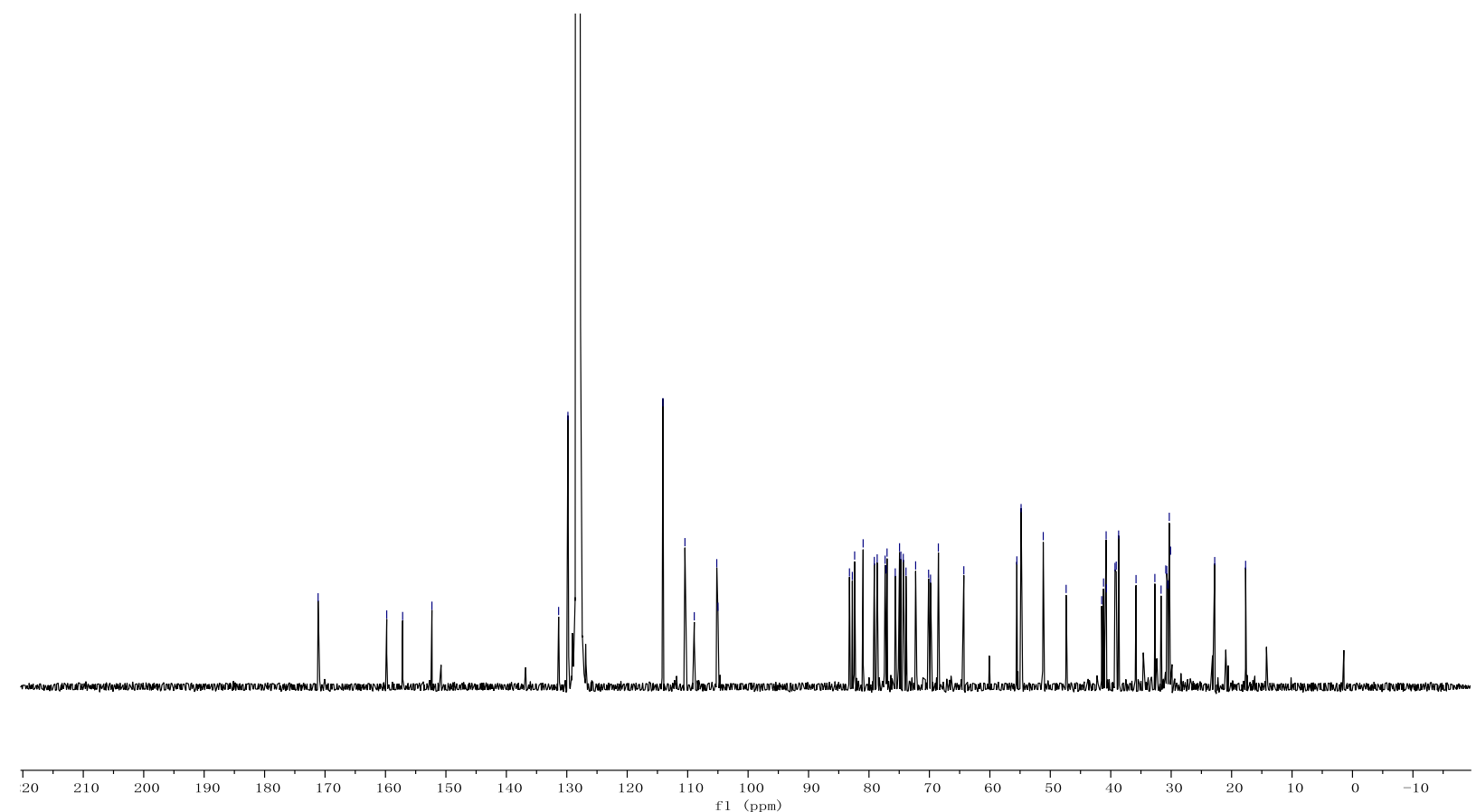
${ }^{1} \mathrm{H}$ NMR $\left(600 \mathrm{MHz}, \mathrm{C}_{6} \mathrm{D}_{6}\right)$ of $\mathbf{5 0 b}$
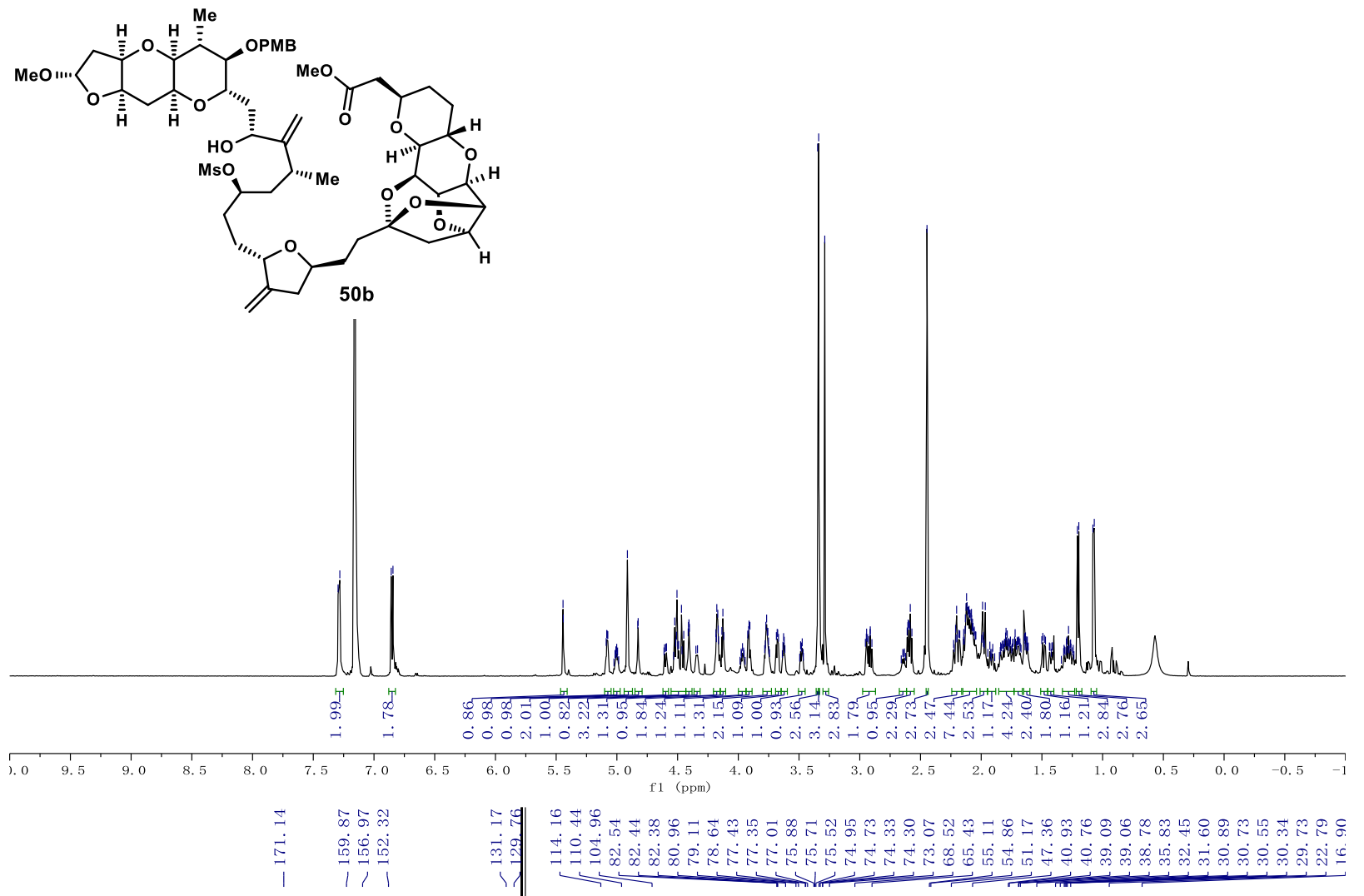

${ }^{13} \mathrm{C}$ NMR (151 MHz, $\left.\mathrm{C}_{6} \mathrm{D}_{6}\right)$ of $\mathbf{5 0 b}$
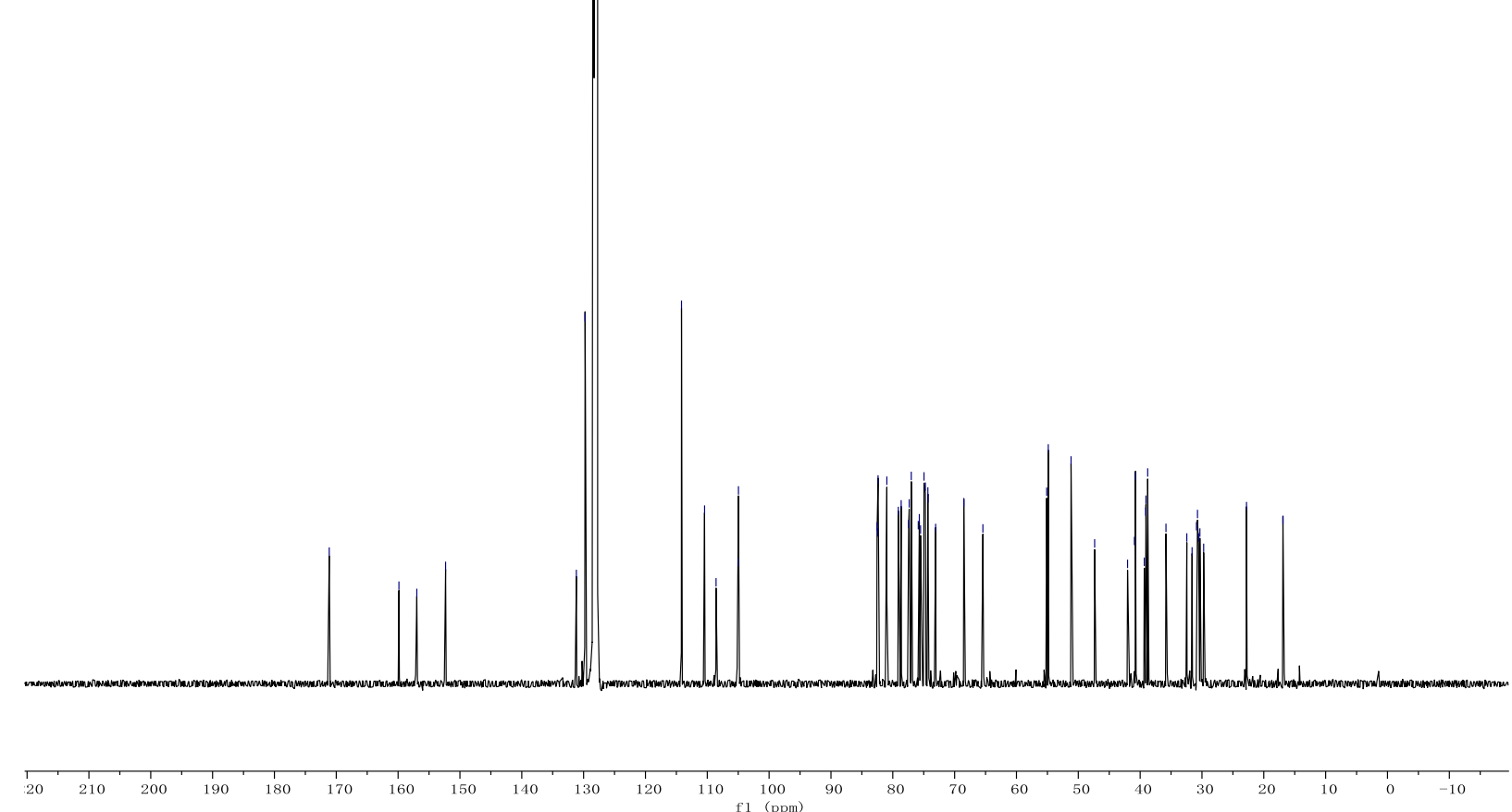


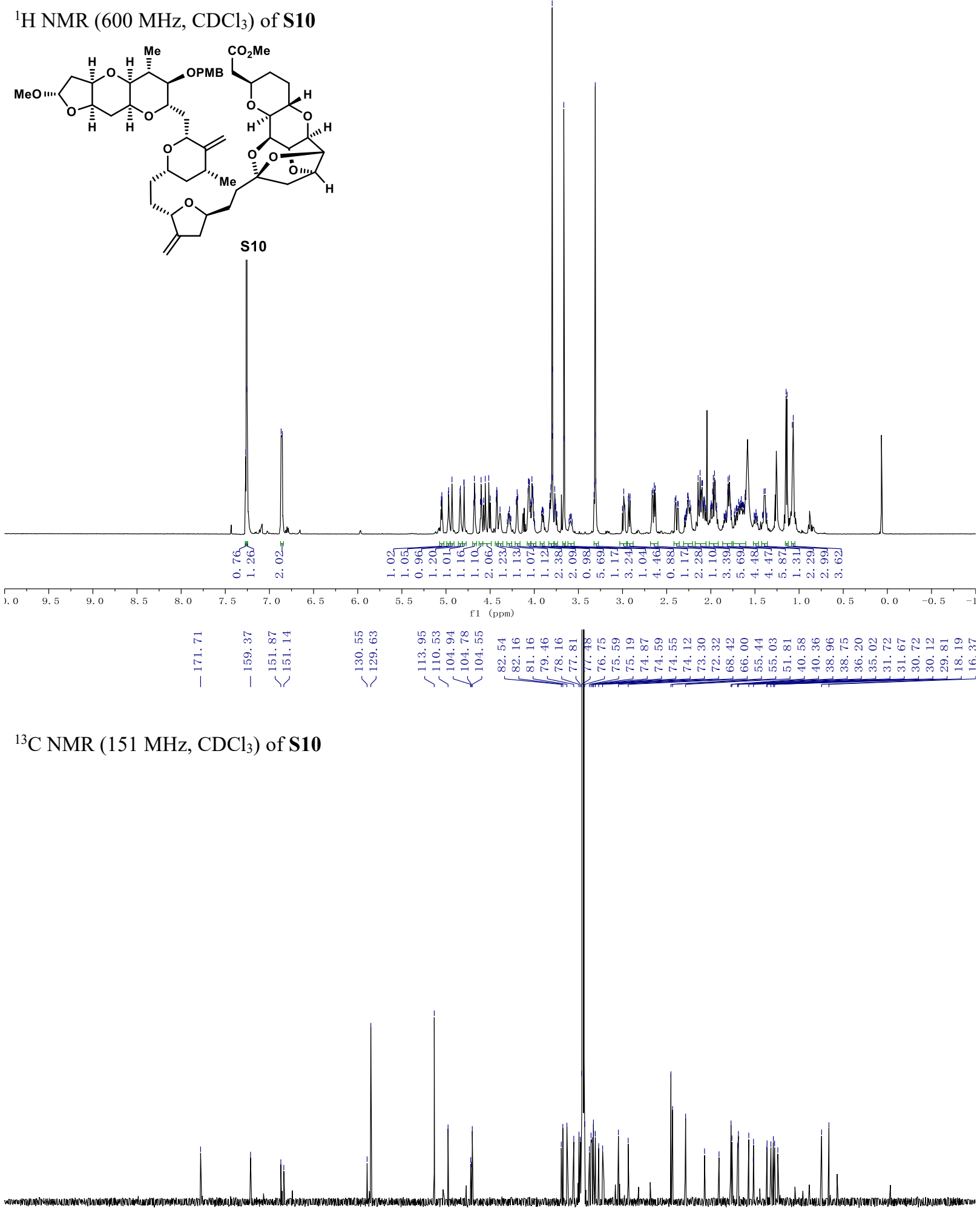

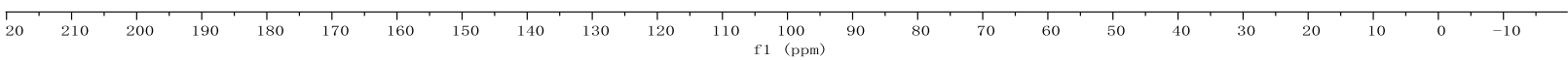


${ }^{1} \mathrm{H}$ NMR $\left(600 \mathrm{MHz}, \mathrm{C}_{6} \mathrm{D}_{6}\right)$ of $\mathbf{5 1}$

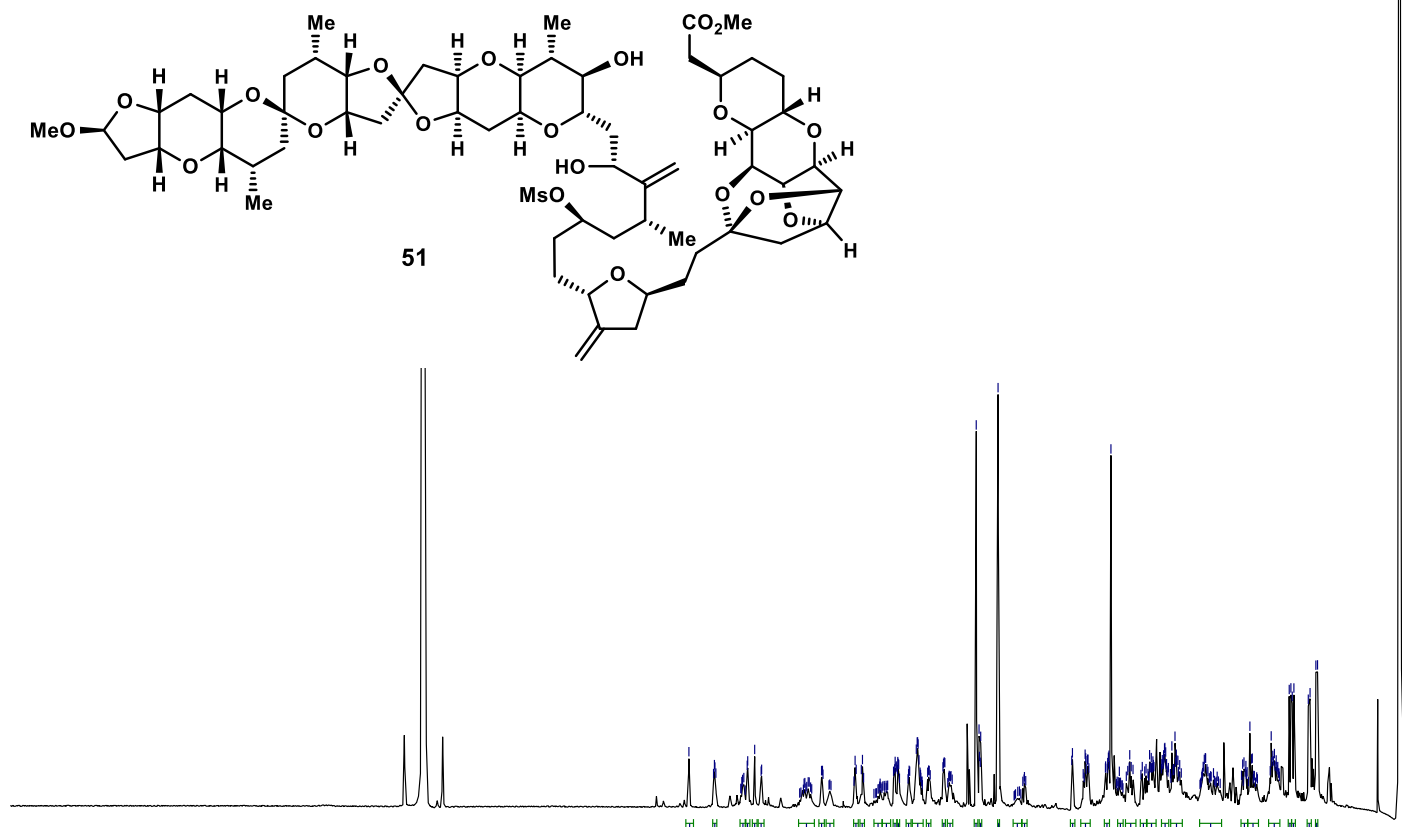

이의

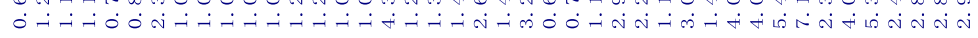

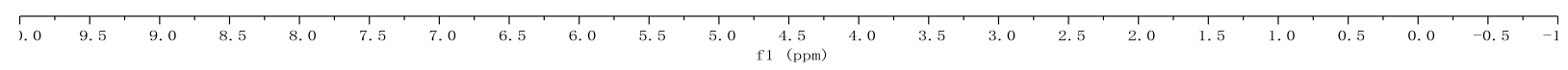

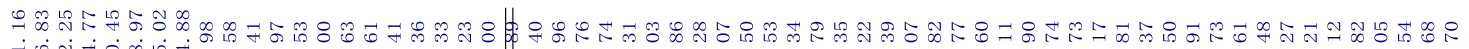

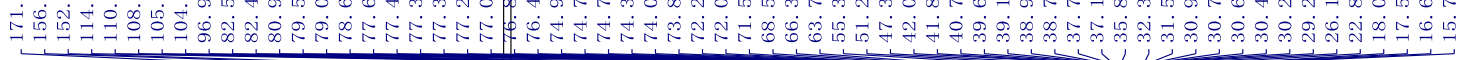

${ }^{13} \mathrm{C}$ NMR (151 MHz, $\left.\mathrm{C}_{6} \mathrm{D}_{6}\right)$ of $\mathbf{5 1}$

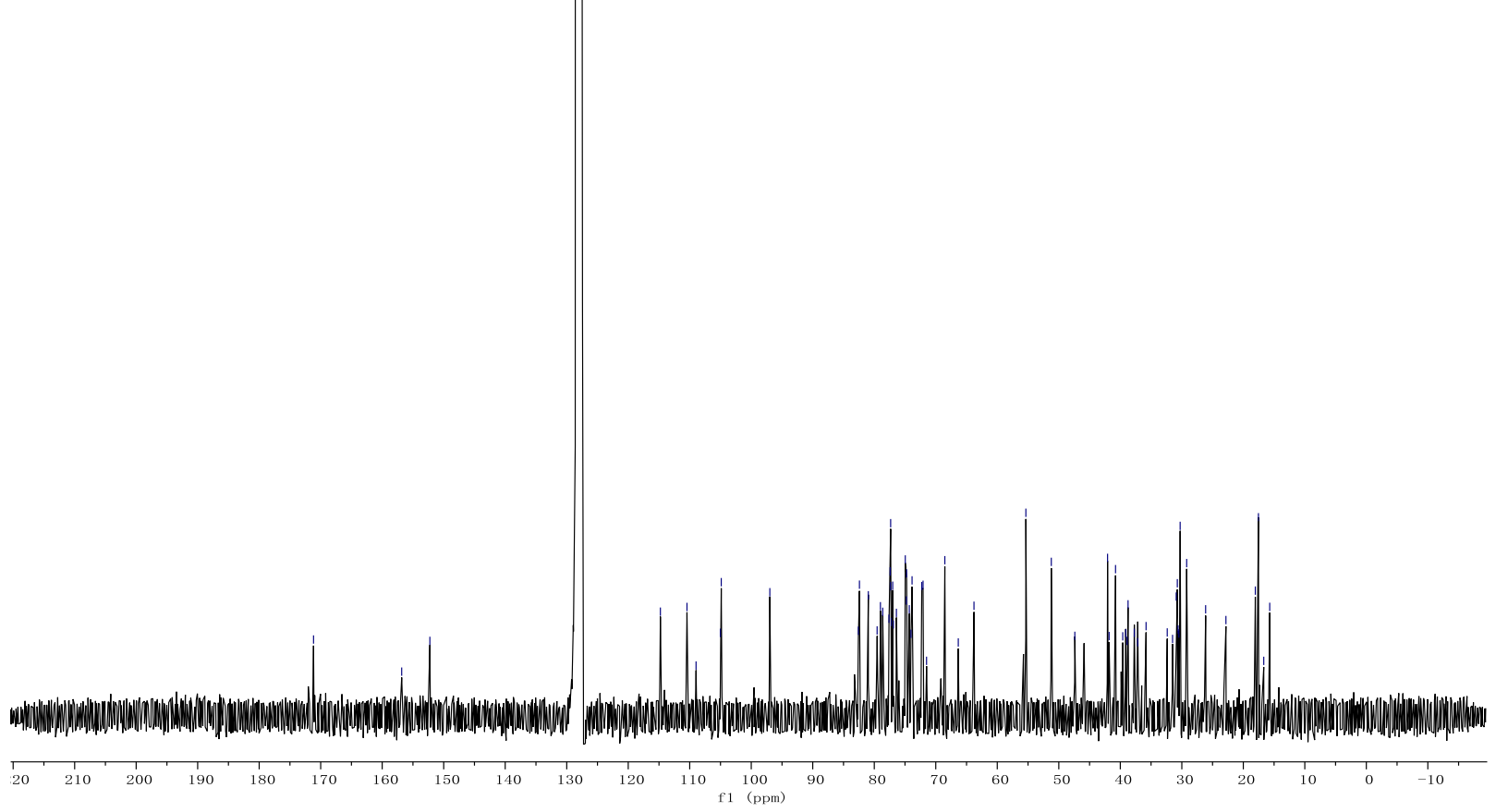


${ }^{1} \mathrm{H}$ NMR $\left(600 \mathrm{MHz}, \mathrm{C}_{6} \mathrm{D}_{6}\right)$ of $\mathbf{5 2 a}$

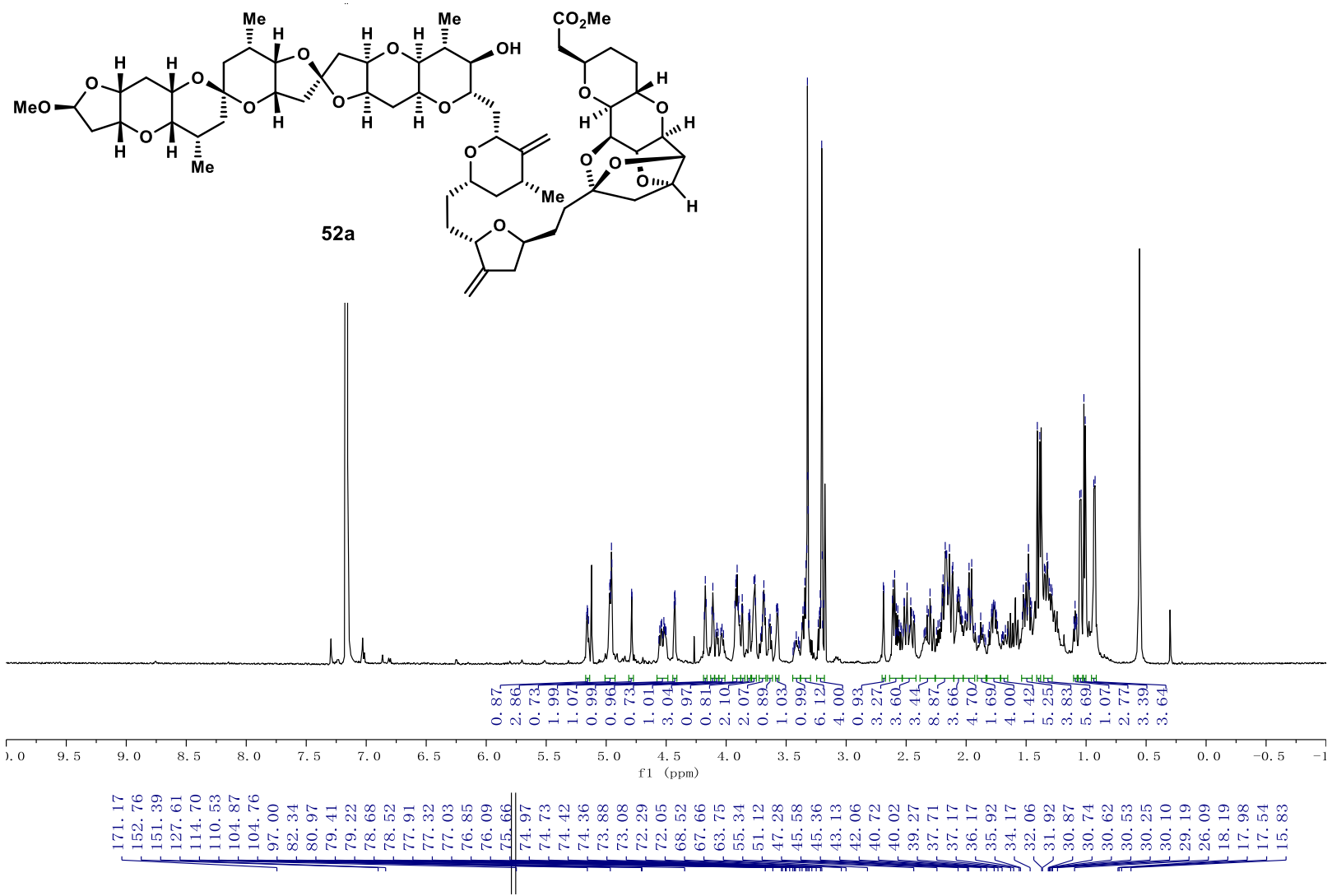

${ }^{13} \mathrm{C}$ NMR $\left(151 \mathrm{MHz}, \mathrm{C}_{6} \mathrm{D}_{6}\right)$ of $\mathbf{5 2 a}$

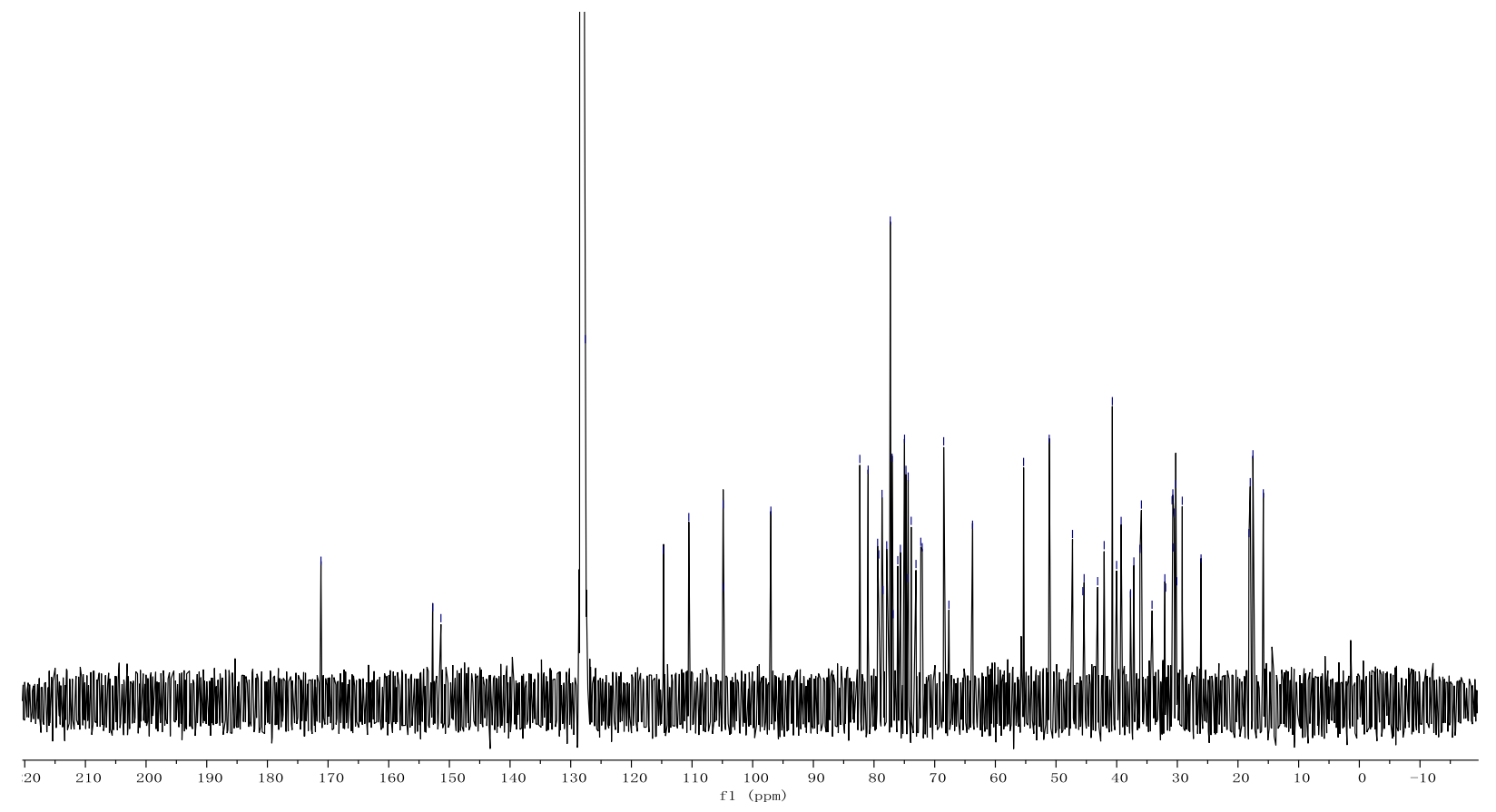


${ }^{1} \mathrm{H}$ NMR $\left(600 \mathrm{MHz}, \mathrm{C}_{6} \mathrm{D}_{6}\right)$ of $\mathbf{5 2}$

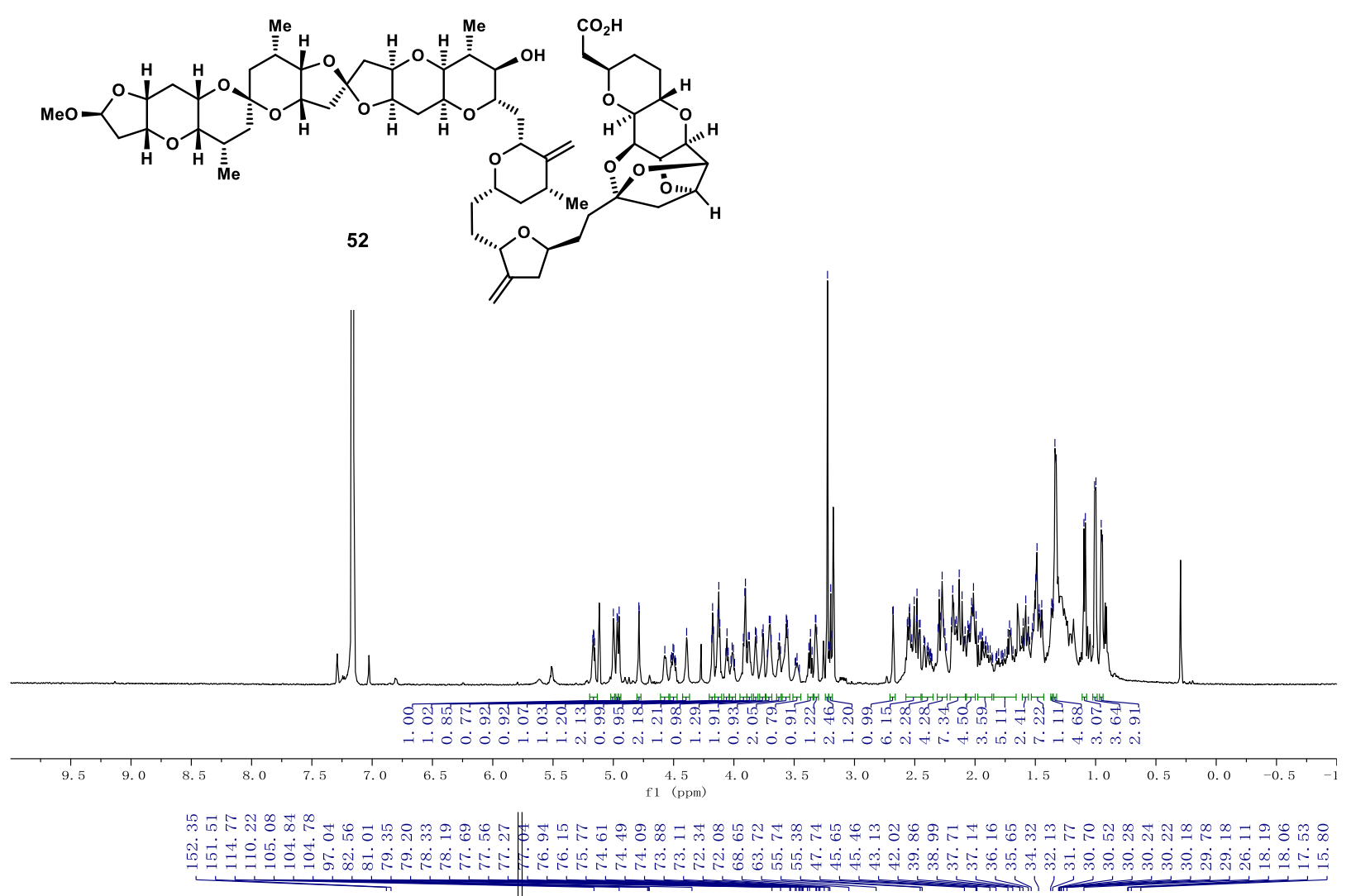

${ }^{13} \mathrm{C}$ NMR (151 MHz, $\left.\mathrm{C}_{6} \mathrm{D}_{6}\right)$ of $\mathbf{5 2}$

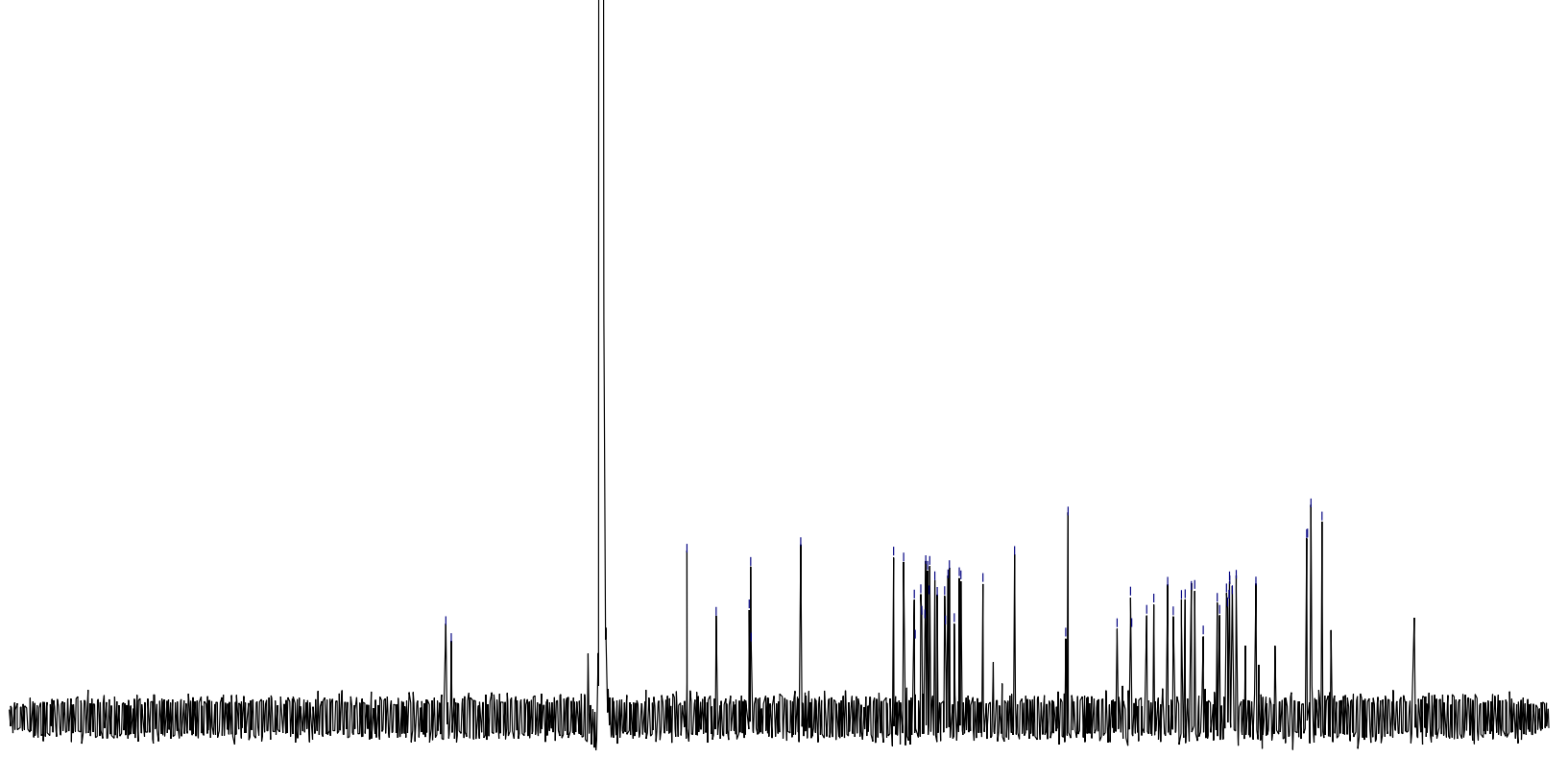

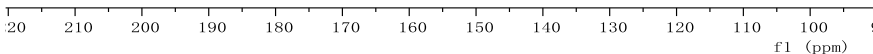


${ }^{1} \mathrm{H}$ NMR $\left(600 \mathrm{MHz}, \mathrm{C}_{6} \mathrm{D}_{6}\right)$ of $\mathbf{5 3}$

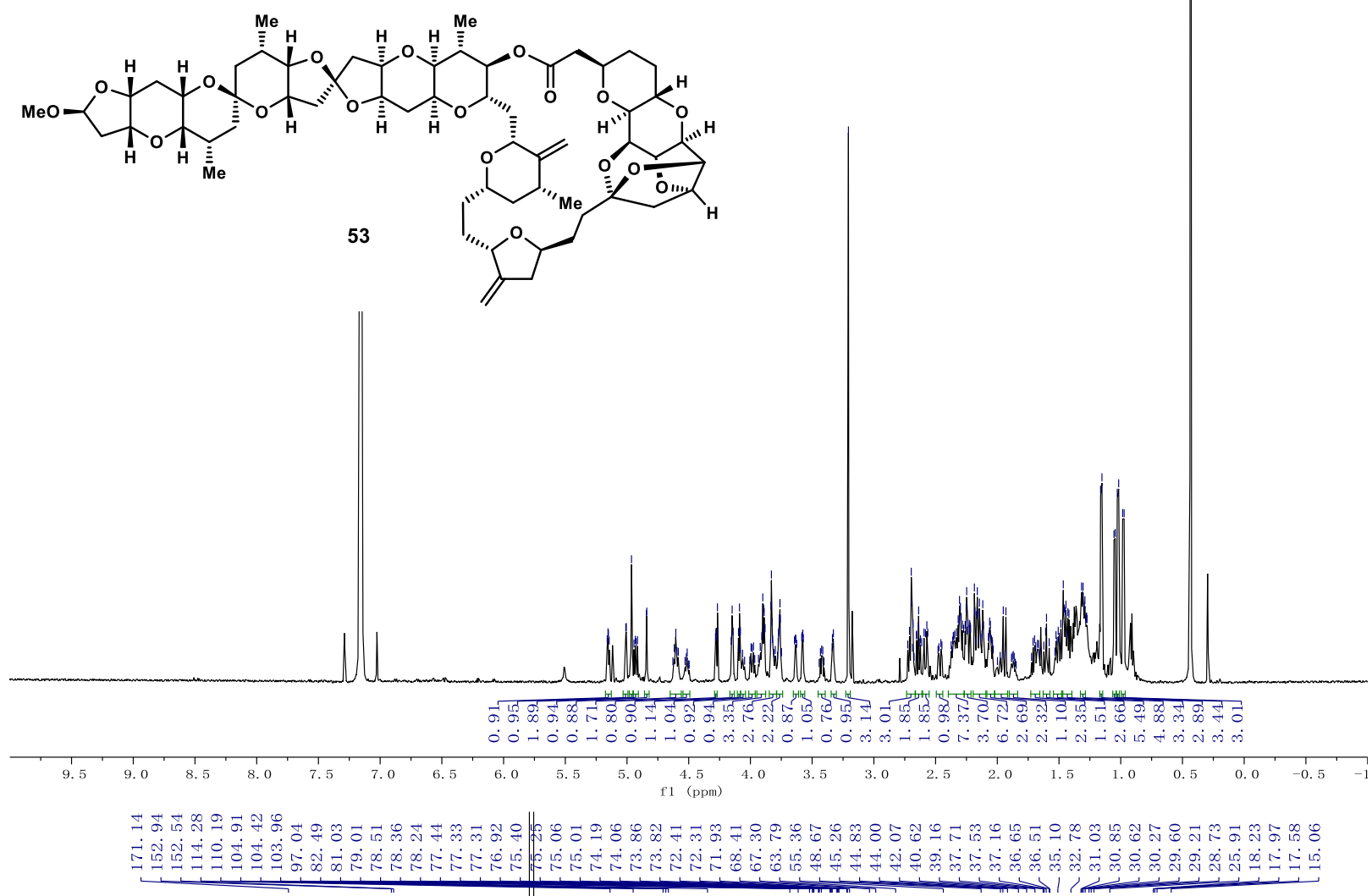

${ }^{13} \mathrm{C}$ NMR $\left(151 \mathrm{MHz}, \mathrm{C}_{6} \mathrm{D}_{6}\right)$ of $\mathbf{5 3}$
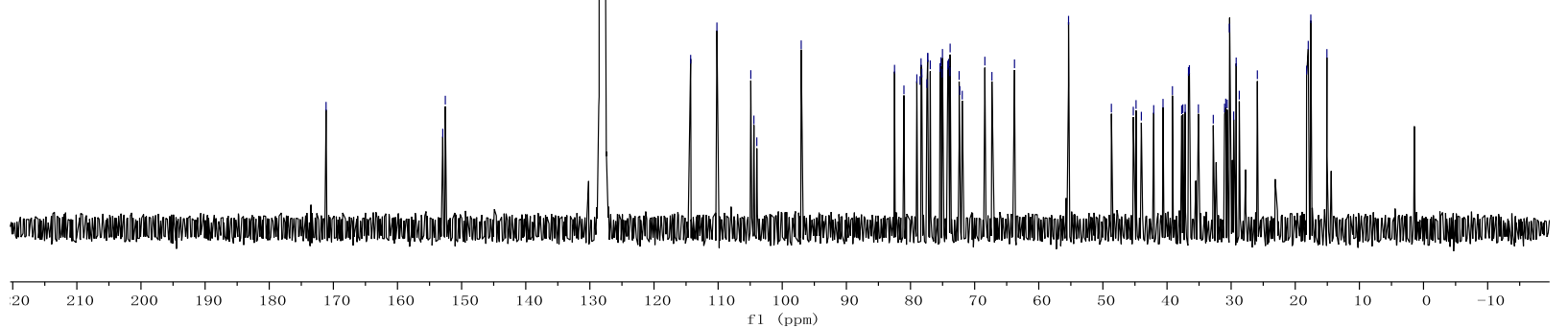
${ }^{1} \mathrm{H}$ NMR (600 MHz, $\left.\mathrm{CD}_{3} \mathrm{OD}\right)$ of norhalichondrin B (2)

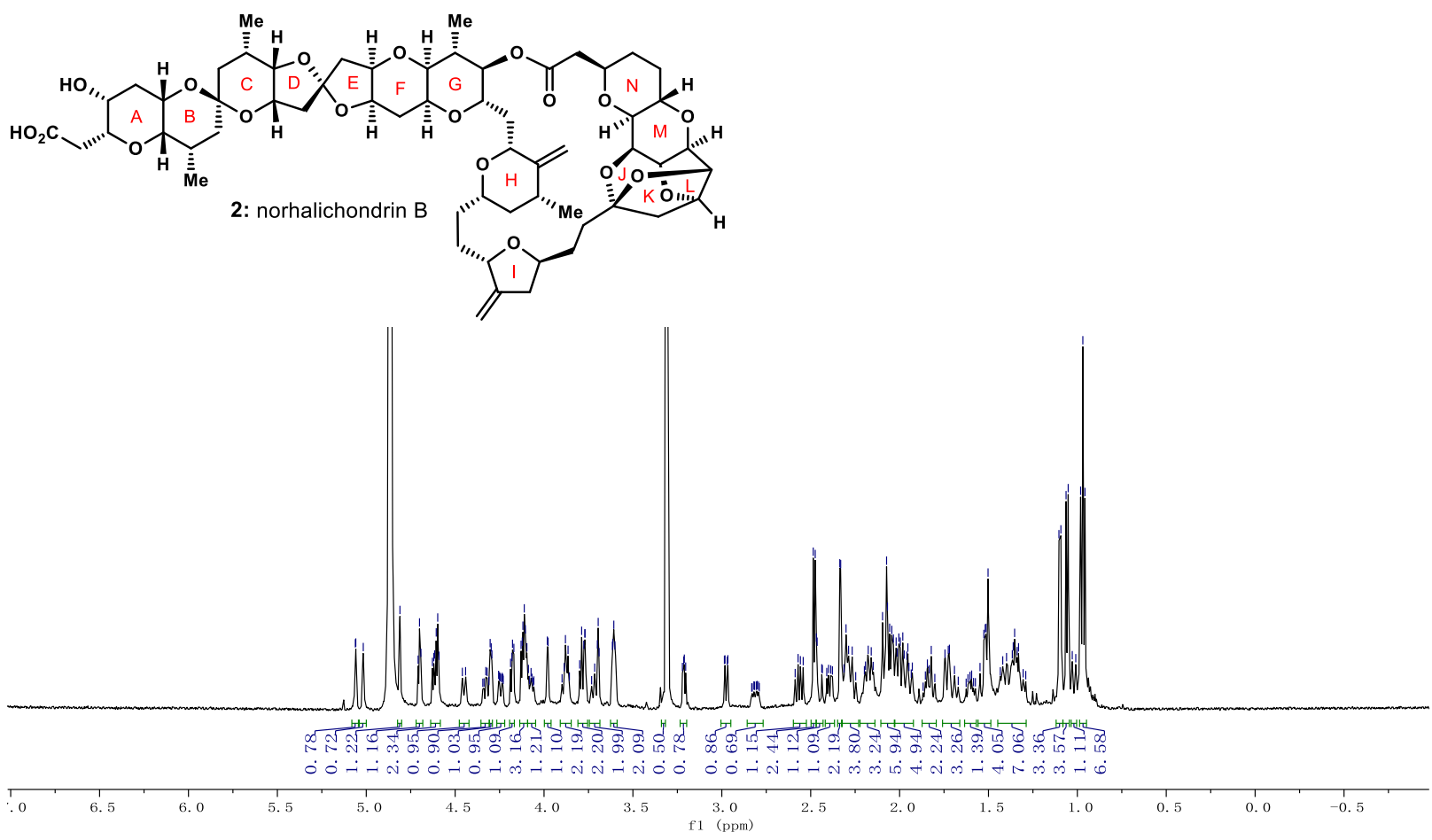

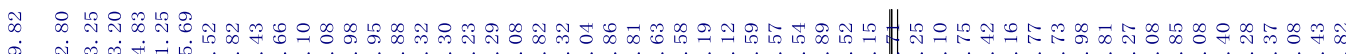

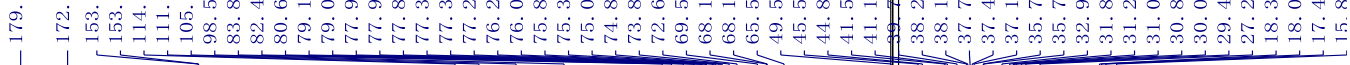

${ }^{13} \mathrm{C}$ NMR (151 MHz, $\left.\mathrm{CD}_{3} \mathrm{OD}\right)$ of norhalichondrin B (2)

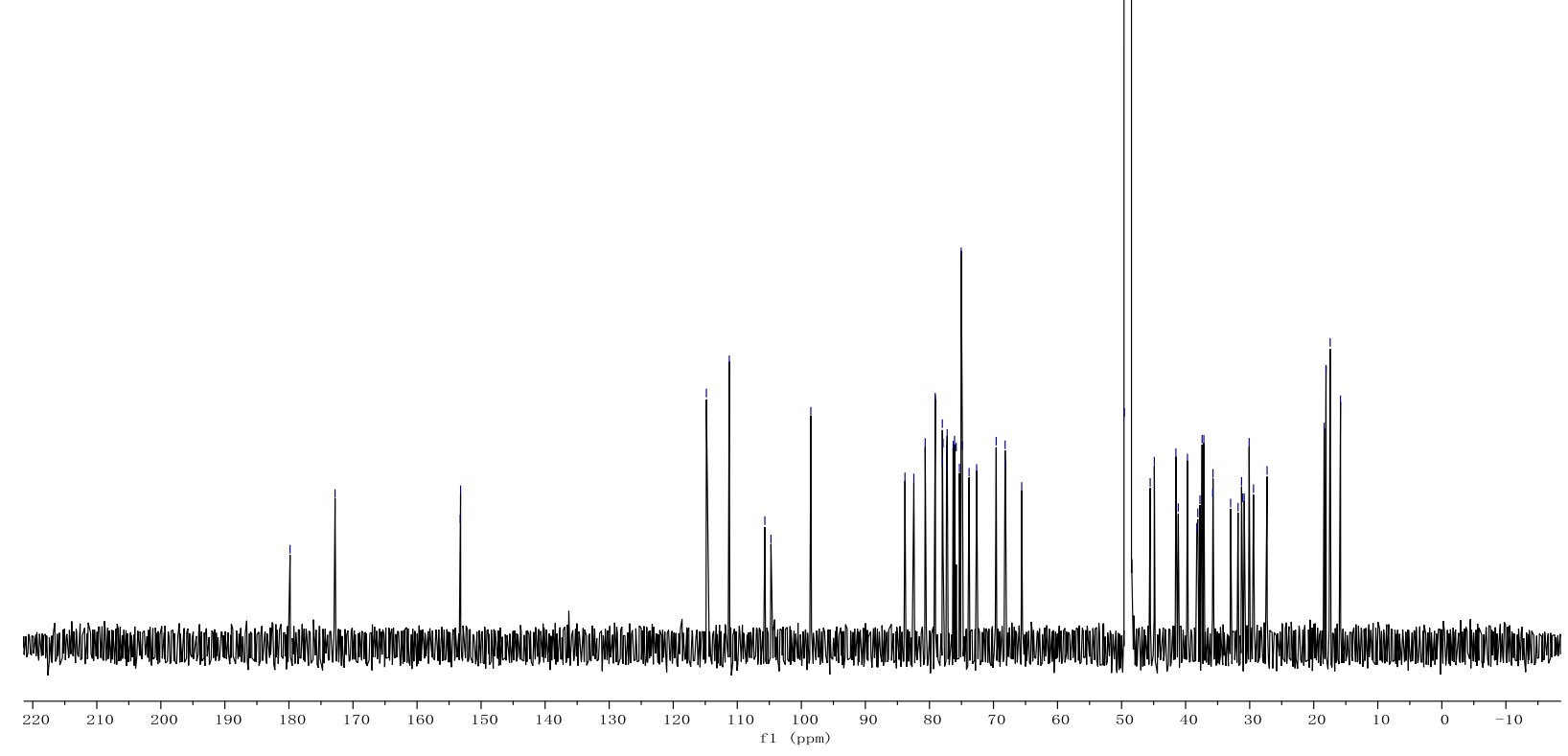


DEPTQ135 NMR (151 MHz, $\left.\mathrm{CD}_{3} \mathrm{OD}\right)$ of norhalichondrin B (2)
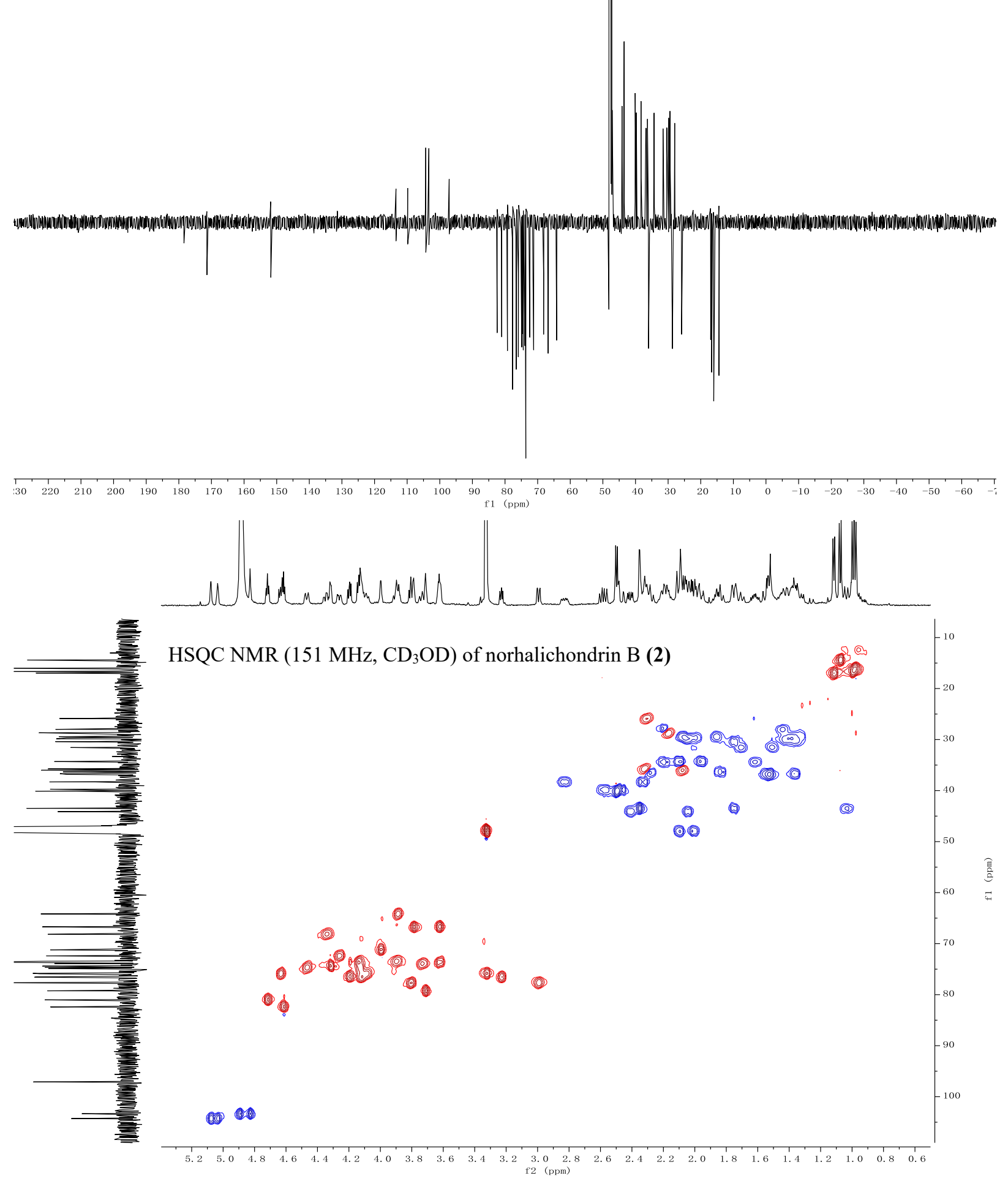


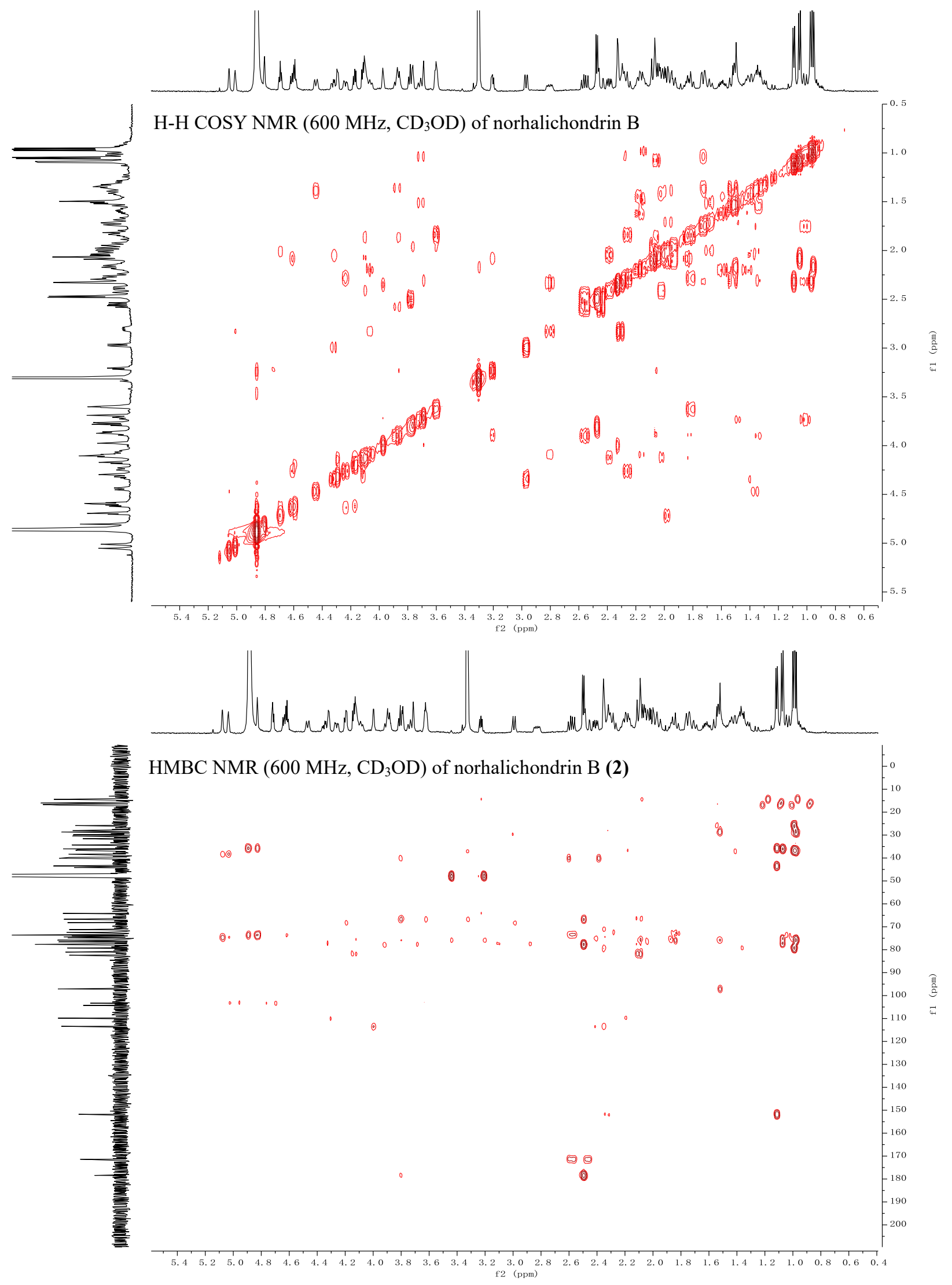




\section{Norhalichondrin B: Comparison of NMR with Kishi's work. ${ }^{8,9}$}

${ }^{1} \mathrm{H}$ NMR (600 MHz, $\mathrm{CD}_{3} \mathrm{OD}$ ) of norhalichondrin B (2), current work.

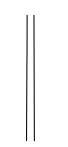

One proton resonance signal overlapping with $\mathrm{H}_{2} \mathrm{O}$ resonance signal

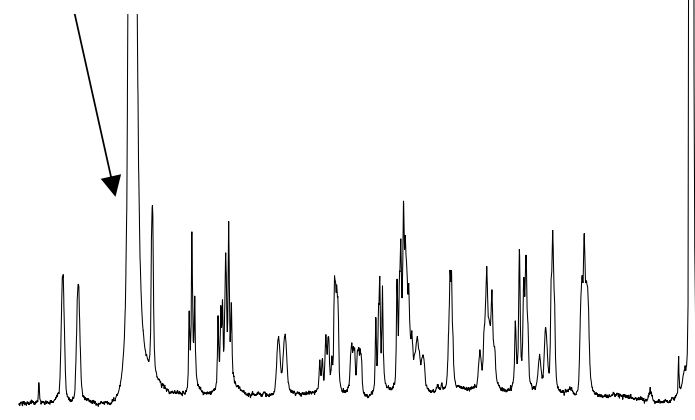

One proton resonance signal overlapping with methanol resonance signal

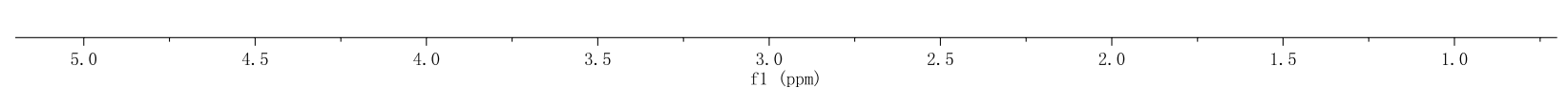

${ }^{1} \mathrm{H}$ NMR (500 MHz, $\mathrm{CD}_{3} \mathrm{OD}$ ) of norhalichondrin B (2),

Y. Kishi and coworkers. ${ }^{8,9}$
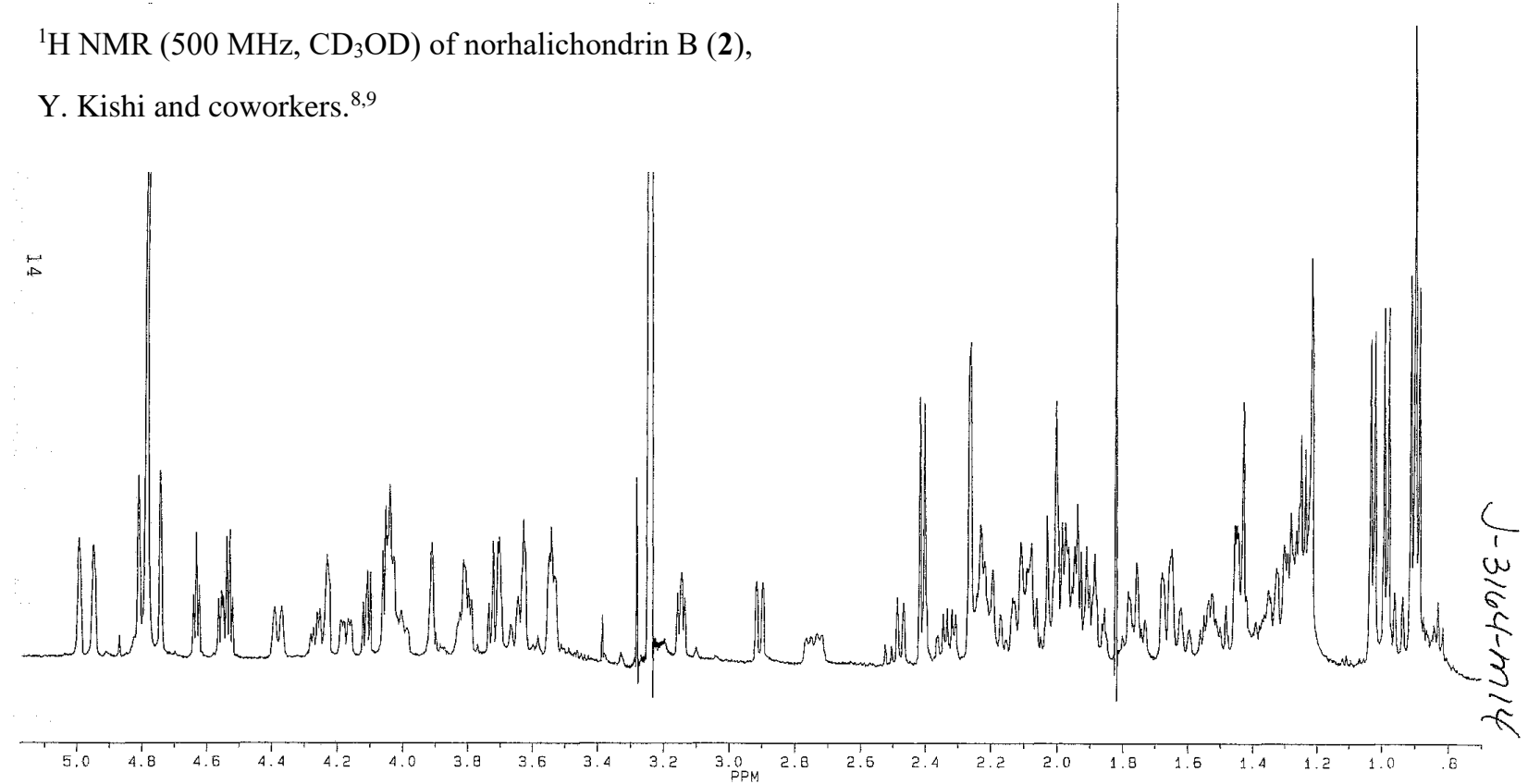


\section{Comparison of NMR with Kishi's work. ${ }^{8,9}$}

${ }^{13} \mathrm{C}$ NMR (600 MHz, $\mathrm{CD}_{3} \mathrm{OD}$ ) of norhalichondrin $\mathrm{B}(2)$, current work.

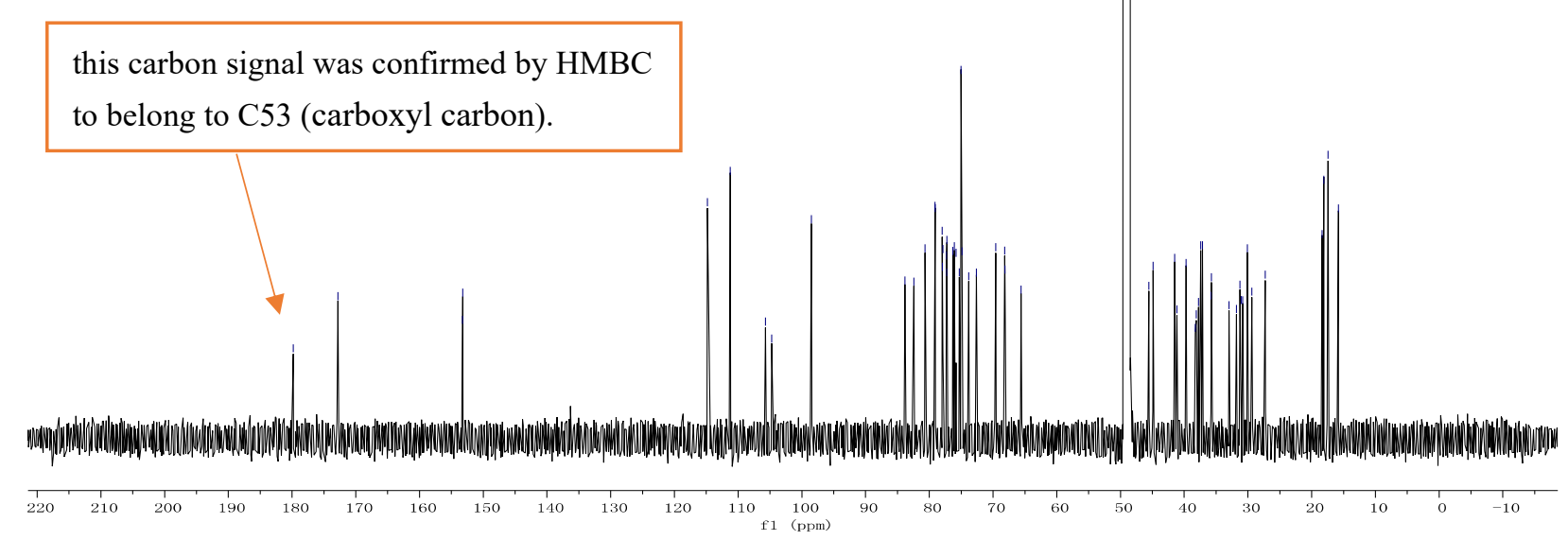

${ }^{13} \mathrm{C}$ NMR (600 MHz, $\mathrm{CD}_{3} \mathrm{OD}$ ) of norhalichondrin B (2),

Y. Kishi and coworkers. ${ }^{8,9}$

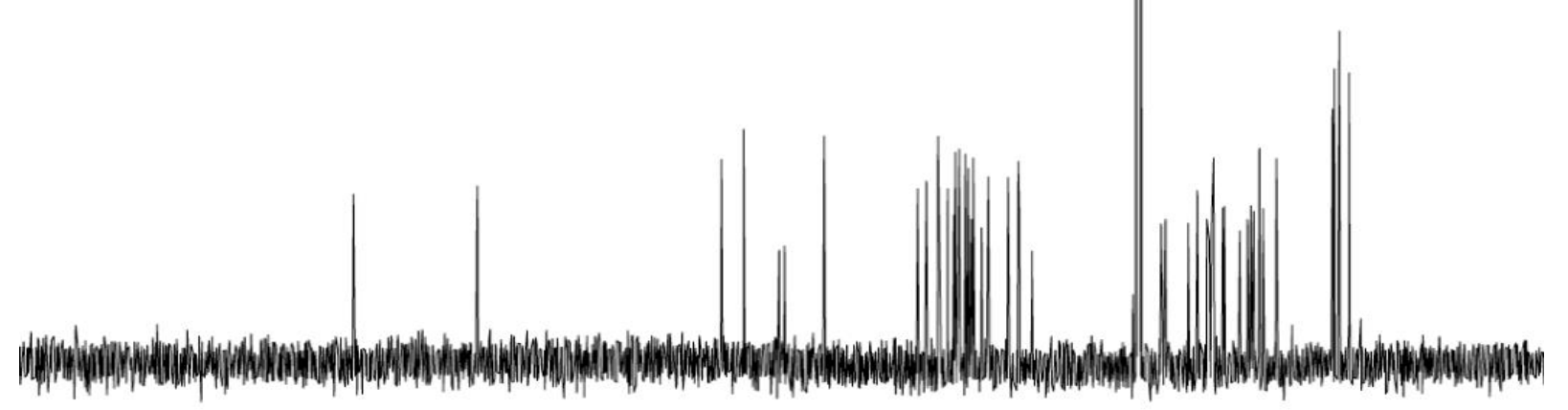

$\begin{array}{llllllllllllllllllllllllllllll}220 & 210 & 200 & 190 & 180 & 170 & 160 & 150 & 140 & 130 & 120 & 110 & 100 & 90 & 80 & 70 & 60 & 50 & 40 & 30 & 20 & 10 & 0 & -10\end{array}$ 


\section{References}

(1) Davis, J. M.; Truong, A.; Hamilton, A. D., Synthesis of a 2,3'; $6^{\prime}, 3^{\prime \prime}$-terpyridine scaffold as an $\alpha$-helix mimetic. Org. Lett. 2005, 7, 5405-5408.

(2) Francis, C. L.; Williamson, N. M.; Ward, A. D., The synthesis of tetrahydroquinolines related to virantmycin. Synthesis 2004, 2685-2691.

(3) Yang, L.; He, G.; Yin, R.; Zhu, L.; Wang, X.; Hong, R., Synthesis of polyketide stereoarrays enabled by a traceless oxonia-Cope rearrangement. Angew. Chem., Int. Ed. 2014, 53, 11600-11604.

(4) Bhoite, S. P.; Kamble, R. B.; Suryavanshi, G. M., An enantioselective synthesis of (+)-hygroline and (+)-pseudohygroline via Keck allylation and CBS reduction. Tetrahedron Lett. 2015, 56, 4704-4705.

(5) Nicolaou, K. C.; Pan, S.; Shelke, Y.; Das, D.; Ye, Q.; Lu, Y.; Sau, S.; Bao, R.; Rigol, S., A reverse approach to the total synthesis of halichondrin B. J. Am. Chem. Soc. 2021, 143, 9267-9276.

(6) Jackson, K. L.; Henderson, J. A.; Motoyoshi, H.; Phillips, A. J., A Total synthesis of norhalichondrin B. Angew. Chem., Int. Ed. 2009, 48, 2346-2350.

(7) Hirata, Y.; Uemura, D., Halichondrins - antitumor polyether macrolides from a marine sponge. Pure Appl. Chem. 1986, 58, 701-710.

(8) Aicher, T. D.; Buszek, K. R.; Fang, F. G.; Forsyth, C. J.; Jung, S. H.; Kishi, Y.; Matelich, M. C.; Scola, P. M.; Spero, D. M.; Yoon, S. K., Total synthesis of halichondrin B and norhalichondrin B. J. Am. Chem. Soc. 1992, 114, 3162-3164.

(9) Yahata, K.; Ye, N.; Ai, Y.; Iso, K.; Kishi, Y., Unified, efficient, and scalable synthesis of halichondrins: Zirconim/Nickel-mediated one-pot ketone synthesis as the final coupling reaction. Angew. Chem., Int. Ed. 2017, 56, 10796-10800. 